\author{
Ursula Quatember \\ Alice Waldner \\ Matthias Pfisterer \\ Maria Aurenhammer
}

\title{
Die Grabung des Jahres 2005 beim Nymphaeum Traiani in Ephesos
}

\section{Fragestellung und Forschungsgeschichte*}

Im Jahr 2005 wurde im Bereich hinter der nordöstlichen Rückwand des Nymphaeum Traiani an der Kuretenstraße (Abb. 1. 2) eine Sondage angelegt. Die damit verbundene Fragestellung bezog sich in erster Linie auf das System der Wasserverteilung, welches hinter der Fassade der Brunnenanlage zu vermuten war ${ }^{1}$.

Das Nymphaeum Traiani (Abb. 3) und seine umgebenden Strukturen wurden in den Jahren 1957 und 1958 unter der Leitung F. Miltners ausgegraben ${ }^{2}$. Aufgrund dessen frühen Todes kam es abgesehen von einer kurzen Erwähnung in Vorberichten aber zu keiner Veröffentlichung der Ergebnisse und Funde. 1962 wurde die Brunnenanlage von H. Pellionis untersucht, der alle Bauglieder aufnahm und eine schematische Rekonstruktion erstellte. $\mathrm{Zu}$ einer abschließenden Publikation des Bauwerks kam es jedoch nicht ${ }^{3}$. In den Jahren 2004-2007 wurde das Nymphaeum Traiani von U. Quatember im Rahmen eines Projekts zu Bauten an der Kuretenstraß $\mathrm{e}^{4}$ neu bearbeitet und eine steingerechte Rekonstruktion erstellt ${ }^{5}$.

Es handelte sich um ein zweigeschossiges Fassadennymphäum mit U-förmigem Grundriss, das - der Stifterinschrift zufolge - von den beiden Ephesiern Ti. Claudius Aristion und seiner Gattin Iulia Lydia Laterane in den Jahren zwischen 102 und $114 \mathrm{n}$. Chr. errichtet wurde ${ }^{6}$. Der Zufluss in das Hauptbecken, dem zur Straße hin ein schmäleres Schöpfbecken vorgelagert war, erfolgte in der Mitte der Rückwand, unterhalb einer überlebensgroßen Statue des Kaisers Traian. Bei der Wasserleitung selbst handelt es sich um eine gemauerte Freispiegelleitung mit rechteckigem Querschnitt und gewölbter Abdeckung ${ }^{7}$. Eine Untersuchung der in situ erhaltenen Reste zeigte Öffnungen in den beiden äußersten Tabernakeln der Rückwand (Abb. 4). Aus dem Baubefund wurde die Hypothese entwickelt, dass hinter der Blendfassade Leitungsstränge vom Zufluss abzweigten und die seitlichen Tabernakel mit Wasser versorgten ${ }^{8}$. Diese These sollte im Jahr 2005 mit einer Grabung hinter der Rückwand des Nymphaeum Traiani überprüft werden. Die Ergebnisse bezüglich des Wasserverteilungssystems sind im Folgenden kurz zusammengefasst. Eine ausführliche Interpretation des

\footnotetext{
* Mein herzlicher Dank gilt F. Krinzinger und S. Ladstätter, ebenso der Projektleiterin H. Thür. S. Swientek sei für seine engagierte Mitarbeit gedankt. W. Prochaska (Montanuniversität Leoben) lieferte wichtige Hinweise zur Bestimmung von Kalkund Sinterproben. Darüber hinaus danke ich J. Auinger für Hinweise. - Abkürzungen und Siglen folgen den Richtlinien des Österreichischen Archäologischen Instituts <http://www.oeai.at/publik/autoren.html>, die zusätzlich verwendeten finden sich am Ende des Beitrags.

${ }^{1}$ Zu ersten Überlegungen bezüglich dieses Wasserverteilungssystems s. Quatember 2006.

${ }^{2}$ Zum Nymphaeum Traiani s. Miltner 1959b, 326-346 Abb. 171. 173-186. Zu den umgebenden Strukturen: Miltner 1960,12 Abb. 8. 9. Zur Grabungstätigkeit F. Miltners an der Kuretenstraße s. Quatember $2005 \mathrm{a}$.

${ }^{3}$ H. Pellionis' schematische Rekonstruktion in Form einer Orthogonalprojektion wurde publiziert bei Bammer 1972-1975, 386 Abb. 9. 10; seine perspektivische Rekonstruktion findet sich bei Scherrer 1995, 119 Abb. 2.

${ }^{4}$ FWF-Projekt »Der Embolos in Ephesos« (Projektnr. P 17617), durchgeführt am Institut für Kulturgeschichte der Antike an der Österreichischen Akademie der Wissenschaften, Projektleitung: H. Thür.

${ }^{5}$ Quatember (in Druckvorbereitung) a.

${ }^{6}$ s. dazu Quatember 2006 sowie Quatember 2007 mit älterer Lit.; Quatember (in Druckvorbereitung) a.

${ }^{7}$ Wiplinger 2006, 26-30 zur sog. Aristion-Leitung.

${ }^{8}$ s. dazu Quatember 2006
} 


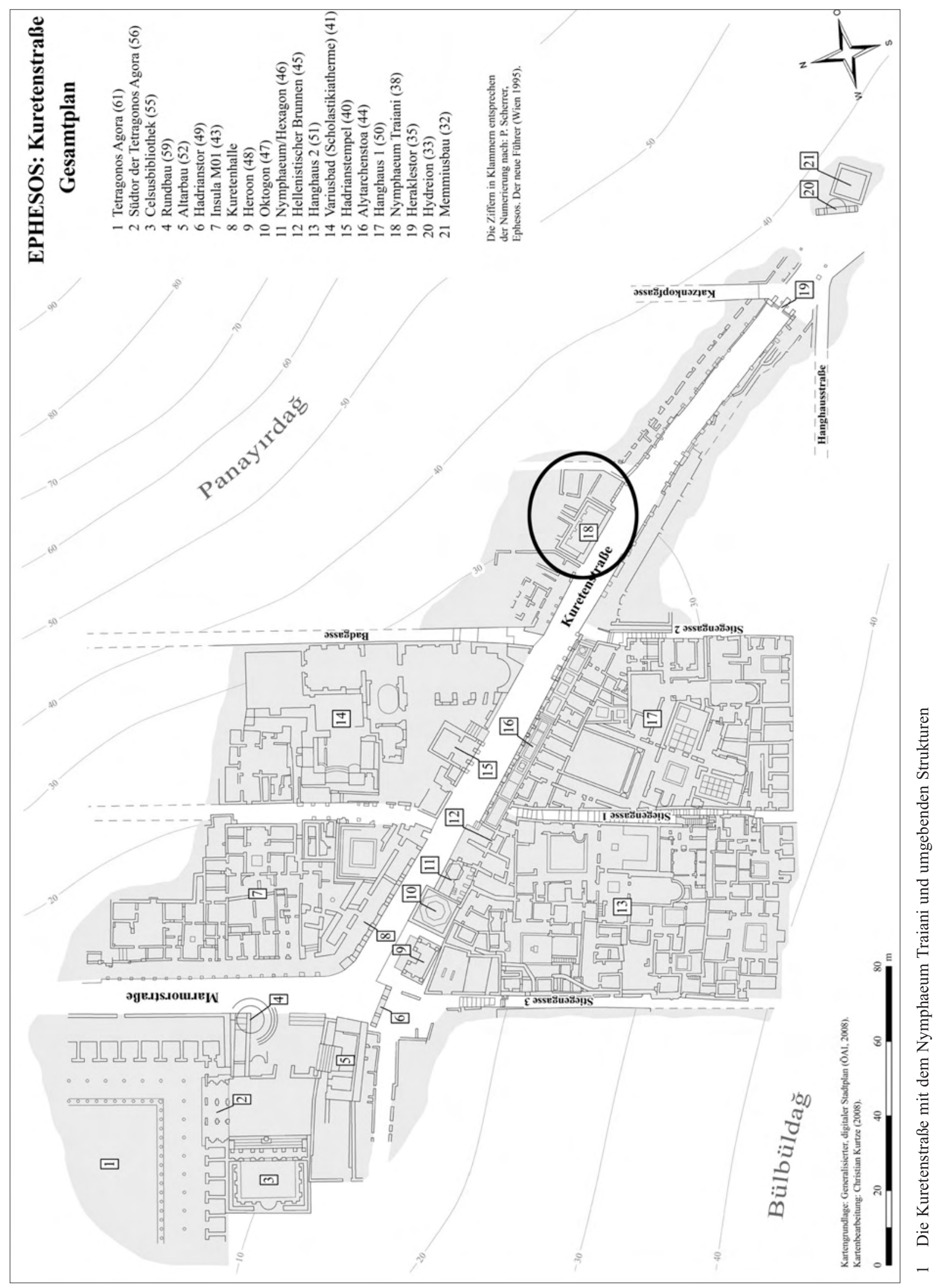


Gesamtkonzepts der Wasserinstallationen wird im Rahmen einer Publikation der Brunnenanlage erfolgen.

Die Sondage des Jahres 2005 führte jedoch nicht nur zu Ergebnissen bezüglich des Nymphaeum Traiani, sondern auch zu Informationen über die Nutzungsgeschichte der umgebenden Strukturen. Diese Fragestellung war aus den Forschungen F. Miltners weitgehend ausgeklammert, da es ihm in erster Linie um die Freilegung der kaiserzeitlichen Gebäude ging, während jüngere Einbauten und Veränderungen oft ohne weitere Dokumentation abgetragen wurden'. Damit können den Ergebnissen von D. Iro, H. Schwaiger und A. Waldner aus den Grabungen in der Nord- und Südhalle im östlichen Abschnitt der Kuretenstraße ${ }^{10}$ weitere Befunde zur Seite gestellt werden, die unsere Kenntnisse über die spätantike Nutzung dieses Areals vertiefen. Erstmals ist es möglich, eine vollständige stratigraphische Abfolge von der Kaiserzeit bis in das 7. Jahrhundert $\mathrm{n}$. Chr. vorzulegen und Keramik sowie Münz- und Skulpturenfunde kontextuell zu behandeln.

Die das Nymphaeum Traini umgebenden Strukturen (Abb. 2) wurden von F. Miltner in seinem posthum erschienenen Bericht nur kurz erwähnt ${ }^{11}$, bislang aber nicht ausführlich untersucht. Bei Miltners Ausgrabungen fanden sich östlich des Nymphaeum Traiani ${ }^{12}$ zwei Porträtköpfe ${ }^{13}$. Ihr genauer Fundkontext ist leider nicht bekannt; ein Zusammenhang mit der Statuenausstattung der Brunnenanlage dürfte jedoch ebenso auszuschließen sein wie eine Verbindung mit dem in einer Aufschüttung angetroffenen Kopf des Doryphoros und anderen Skulpturenfragmenten ${ }^{14}$, da diese auf einem wesentlich tieferen Niveau gefunden wurden, welches F. Miltner bei seinen >Freilegungsarbeiten $<$ bis zu dem von ihm angenommenen `Fußbodenniveau< nicht erreichte.

Eine bauhistorische Untersuchung des Bereichs nordöstlich des Nymphaeum Traini ist zwar nicht Gegenstand dieses Beitrags, dennoch sei der Baubefund hier zusammengefasst: In diesem Areal treffen Strukturen aufeinander, die auf unterschiedliche Straßenverläufe Bezug nehmen (Abb. 1. 2). Die Rückwand der Brunnenanlage sowie die daran anschließende Zungenmauer M 3 sind am Verlauf der Kuretenstraße bzw. in rechtem Winkel zu dieser orientiert, die übrigen Mauern dieses Bereichs hingegen beziehen sich auf den orthogonalen Raster der hellenistisch-römischen Stadt ${ }^{15}$. Eine L-förmige Mauer trennt einen rechteckigen Bereich von etwa $6 \times 4,5 \mathrm{~m}$ ab, der in seiner letzten Nutzungsphase einen unregelmäßig verlegten Plattenboden aus hellgrauem, fast weißem Marmor besaß, bei dem es sich, der teilweise vorhandenen Profilierung in Form von Längsrillen nach zu schließen, offenbar um sekundär verwendete Wandverkleidungsplatten handelte. In der Nordostecke befand sich ein Treppenaufgang in das Obergeschoss. Der Bereich südlich der Mauer war z. T. mit einem wesentlich gröberen Steinpflaster bedeckt, dessen westlichen Abschluss eine Türschwelle mit Einlassungen für ein Gewände sowie Türpfannen bildet.

\section{Befundbeschreibung und Interpretation}

Die Befundsituation (Abb. 5) lässt sich im Wesentlichen in vier Abschnitte unterteilen, die zur besseren Übersicht hier knapp vorgestellt werden sollen:

- Im untersten Bereich der Sondage wurden im Westteil Tonrohrleitungen angetroffen (Abb. 7. 8), die in Zusammenhang mit der Wasserführung des zwischen 102 und 114 n. Chr. errichteten Nymphaeum Traiani stehen. Die Verfüllung zwischen den Tonrohren ist in severische Zeit zu datieren; sie könnte von einer

\footnotetext{
${ }^{9}$ Vgl. Quatember 2005a. Man denke beispielsweise an die sog. Schuttsperre zur Abstützung des Hangschutts im Bereich des sog. Hadrianstempels an der Kuretenstraße, die nur im handschriftlichen Tagebuch erwähnt, mit wenigen Photos dokumentiert und dann abgerissen wurde, vgl. dazu Auinger 2009.

${ }^{10}$ s. dazu Iro - Schwaiger - Waldner 2009.

${ }_{11}$ Miltner 1960, 12; Miltner 1959a, 35.

12 Diese Fundortangabe bei Miltner 1960, 12 ist identisch mit den Angaben im handschriftlichen Tagebuch vom 6. Oktober 1958 (Archiv des ÖAI, Wien).

${ }^{13}$ Männliche Porträtbüste mit Einsatzkopf, vgl. Miltner 1960, 12. Abb. 8; İnan - Rosenbaum 1966, 139 Nr. 173 Taf. 101, 3; 102, 1-2; Bammer - Knibbe - Fleischer 1974, 15 f.; Aurenhammer (in Druckvorbereitung). Zu einem männlichen Porträt mit über den Kopf gezogenen Mantel s. İnan - Rosenbaum 1966, 141 Nr. 177 Taf. 104, 3-4; İnan - Alföldi-Rosenbaum 1979, 5; Aurenhammer (in Druckvorbereitung).

${ }^{14}$ s. u. Kapitel 5 (M. Aurenhammer).

${ }^{15}$ Scherrer 2001; Groh 2006, jeweils mit weiterer Lit.
} 


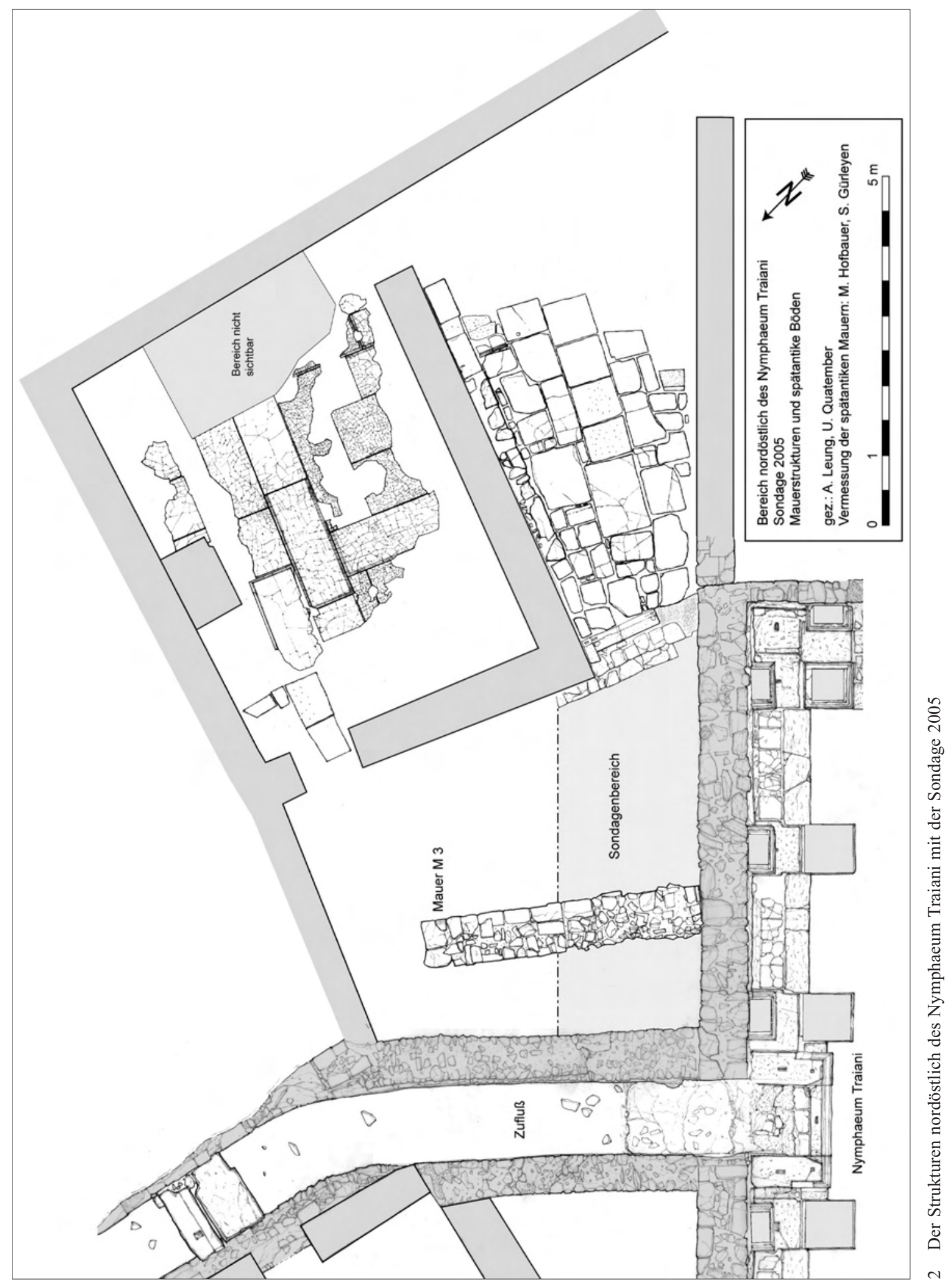




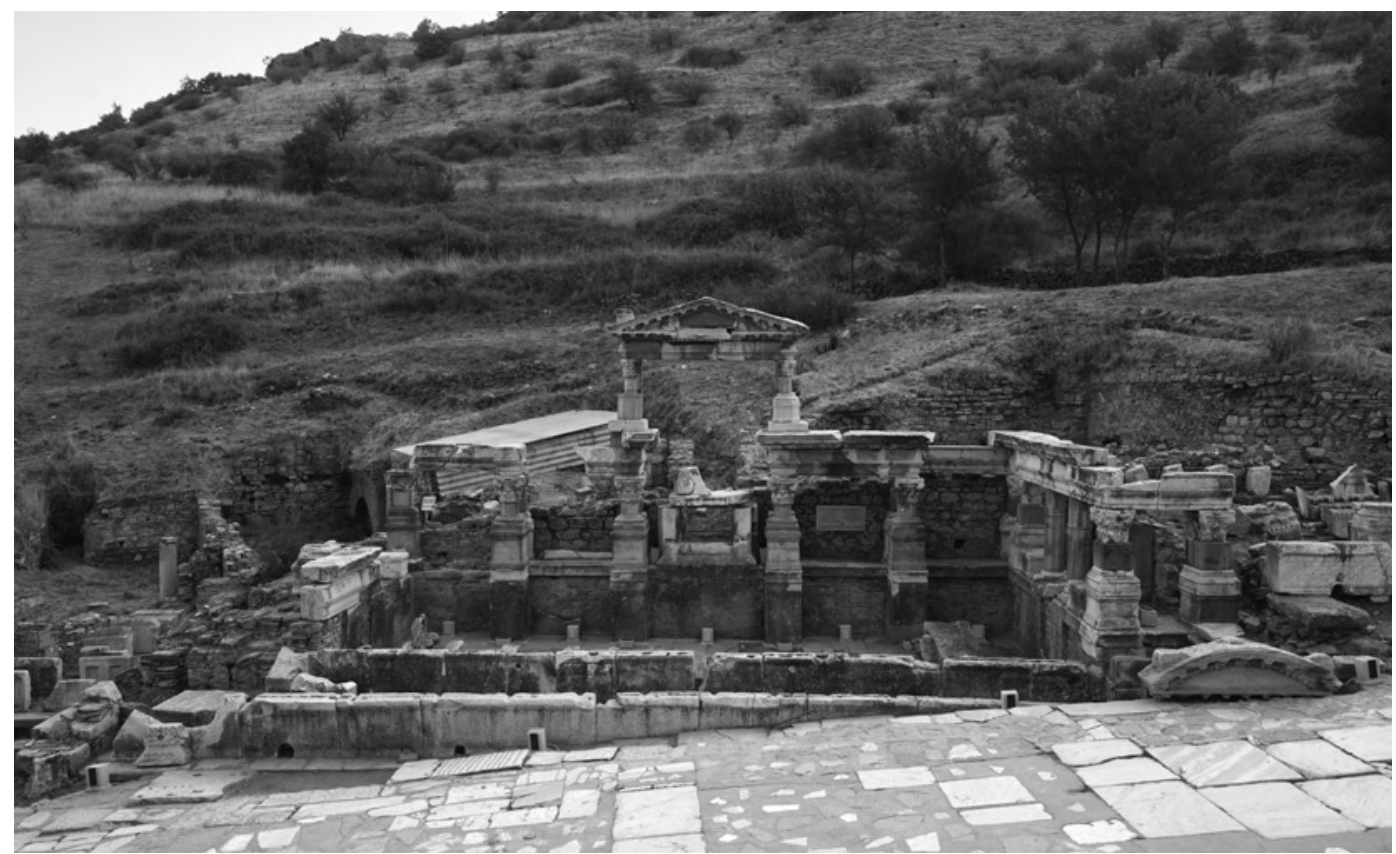

3 Das Nymphaeum Traiani (2006)

Reparatur der ursprünglichen Anlage in diesem Bereich stammen. Im Ostteil bildet ein qualitativ hochwertiger Marmorplattenboden die Grabungsgrenze. Es handelt sich dabei sicherlich um das kaiserzeitliche Gehniveau der Gebäudestrukturen hinter dem Nymphäum. Dieser Plattenboden dürfte wenig später entstanden sein als die darunter verlaufenden Rohrleitungen. Die Verbindung zu den östlich anschließenden Baustrukturen wurde durch eine später angelegte, halbrunde Treppe aus Ziegeln hergestellt.

- In der ersten Hälfte des 5. Jahrhunderts kam es zu einer zumindest teilweisen Aufgabe der Strukturen hinter dem Nymphäum. Eine Planierschicht wurde eingebracht und in der Südwestecke hinter der Rückwand des Nymphäums in einer Grube Kalk gelöscht, wofür Wasser aus dem Zufluss zum Nymphaeum Traiani entnommen und in ein rechteckiges Steinbecken umgeleitet worden war (Abb. 10).

- Im Laufe des 6. Jahrhunderts wurden das Steinbecken verfüllt und erneut mehrere Planierschichten eingebracht. In einer Grube fand sich umgelagerter Werkstattschutt des späten 6./frühen 7. Jahrhunderts, der neben Gusstiegeln u. a. auch mehrere Statuenfragmente umfasste, darunter das Kopffragment einer antoninischen Kopie des Doryphoros (S 1). Diese Aufschüttungen bilden die Unterkonstruktion für den Boden der Baustrukturen des 7. Jahrhunderts n. Chr., der selbst nicht mehr erhalten ist.

- Die letzte nachgewiesene Bauaktivität in diesem Bereich stellt die Mauer M 3 dar (Abb. 2. 12), für die aufgrund der Keramik der unmittelbar darunterliegenden Schicht sowie aus der Mauer selbst als terminus post quem die erste Hälfte des 7. Jahrhunderts n. Chr. gelten kann.

Im Folgenden werden die einzelnen Befunde mit ihrer Stratigraphie vorgestellt. Die Tabelle dient jeweils der besseren Übersicht und den Querverweisen auf die Abschnitte zu Keramik, Münz- und Skulpturenfunden. Zum besseren Verständnis sei kurz der Grabungsablauf skizziert: Zunächst wurde Schnitt 1 West bzw. Ost zu beiden Seiten der Mauer M 3 angelegt. Schnitt 2 befand sich östlich davon. In weiterer Folge wurden sowohl der Steg zwischen Schnitt 1 und 2 als auch M 3 zwischen den beiden Teilen von Schnitt 1 nach Dokumentation der Profile - abgetragen ${ }^{16}$, sodass im Endbefund eine durchgehende Fläche entstand (Abb. 2).

${ }^{16}$ Die Nummerierung der stratigraphischen Einheiten (SE) erfolgte fortlaufend für beide Sondagen; einzelne Straten konnten später zur Übereinstimmung gebracht werden, während hingegen andere Nummern nur in einem der beiden Schnitte vorkommen. Diese Vorgangsweise schien aus arbeitsökonomischen Gründen sinnvoll, führt in manchen Fällen aber zu einer inversen Nummerierung der Schichten. Die Besprechung der Befunde erfolgt gemäß der stratigraphischen Abfolge beginnend mit den ältesten nachweisbaren Befunden in diesem Bereich. 


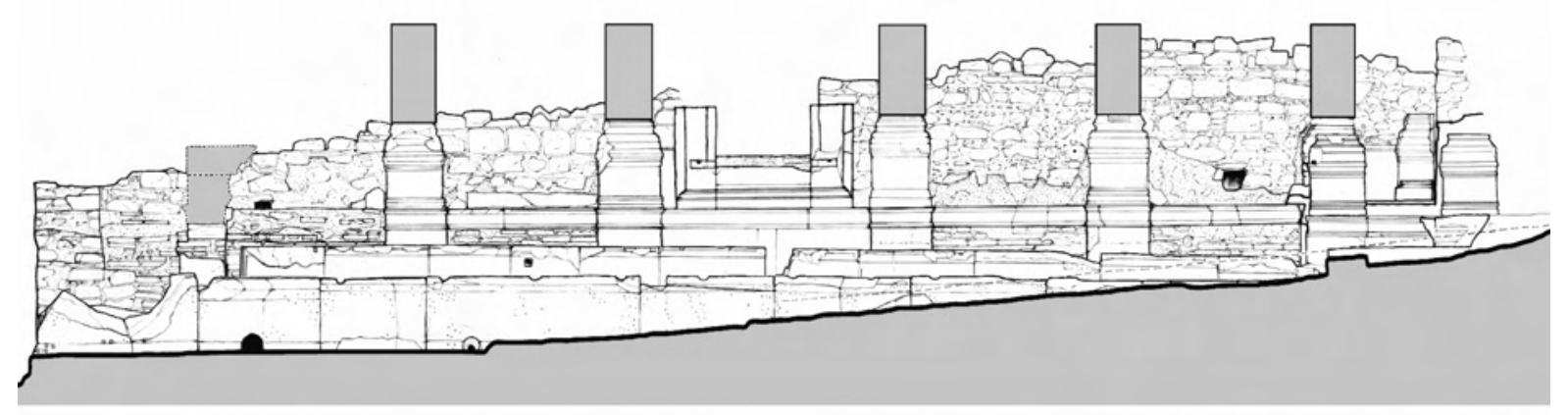

Nymphaeum Traiani, in situ erhaltener Baubefund

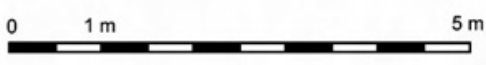

4 Die in situ erhaltenen Reste des Nymphaeum Traiani mit den Öffnungen in den beiden äußeren Tabernakeln der Rückwand

2.1 Befunde in Zusammenhang mit dem Nymphaeum Traiani

\begin{tabular}{|c|l|l|c|c|}
\hline SE & \multicolumn{1}{|c|}{ FK } & \multicolumn{1}{|c|}{ Kurzcharakterisierung } & Datierung & Querverweise \\
\hline 35 & $\begin{array}{l}58,60,63,64,65, \\
66,110,111\end{array}$ & $\begin{array}{l}\text { Verfüllung zwischen den Tonrohrleitungen: Bauschutt } \\
\text { mit Wandmalereifragmenten und Mosaiksteinen }\end{array}$ & severisch & Mü 2005/47, /48 \\
\hline 33 & 56,109 & $\begin{array}{l}\text { Mehrschichtiger Estrich als Gehniveau über den Ton- } \\
\text { rohrleitungen mit wenig, kleinteilig gebrochener Kera- } \\
\text { mik, flächig im Westteil des Schnittes }\end{array}$ & - & - \\
\hline
\end{tabular}

Im Westteil der Sondage wurden insgesamt drei Tonrohrleitungen angetroffen (Abb. 6. 7). Diese zweigen von der Zuleitung zum Nymphaeum Traiani ab, einer gemauerten Freispiegelleitung mit rechteckigem Querschnitt. Der Zufluss zur Brunnenanlage erfolgt auf wesentlich höherem Niveau (Sohle des Zuflusskanals: 21,96 m abs. H; Abzweigung auf max. 22,24 m abs. H) als das kaiserzeitliche Bodenniveau hinter der Nymphäumsrückwand (Plattenboden: 21,23 m abs. H). Dementsprechend befinden sich unmittelbar an der Außenseite des Zuflusses steile Fallrohre (Abb. 8), die unterhalb des einstigen Fußbodens in Leitungen mit schwachem Gefälle umknicken. Zwei Rohrleitungen verlaufen unterhalb des Marmorplattenbodens, der im Ostteil die Grabungsgrenze der Sondage darstellt.

Bei der die Rohre umgebenden Schicht SE 35 handelt es sich teilweise um Bauschutt mit Wandmalereifragmenten und Mosaiksteinen ${ }^{17}$. Aufgrund der Datierung der Keramikfunde ist nur eine allgemeine Einordnung vom 2. Jahrhundert n. Chr. bis spätestens in severische Zeit möglich. Die Fundmünze Mü 2005/4718 ist Iulia Domna zuzuordnen und verweist damit in severische Zeit. Daraus ergibt sich jedoch wahrscheinlich kein terminus post quem für das gesamte Leitungssystem hinter dem Nymphäum, sondern nur für seine letzte Reparaturphase in diesem Bereich.

Aus dieser Datierung und dem architektonischen Befund lässt sich Folgendes für die Wasserleitung des Nymphaeum Traiani und die chronologische Abfolge der Bauaktivitäten rekonstruieren: Gleichzeitig mit seiner Nutzung als Brunnen erfüllte das Nymphaeum Traiani eine Art Verteilerfunktion, indem Wasser vom Hauptzufluss noch vor dem Eintritt in die Brunnenfassade in andere Bereiche bzw. Gebäude umgeleitet wurde. Dies zeigen die beiden tiefer gelegenen Stränge, die vom Nymphäum in östlicher bzw. nordöstlicher Richtung abzweigen und somit nicht in Verbindung mit der Wasserversorgung der seitlichen Brunnennischen gesetzt werden können. Die Vorgängerbebauung dieses Bereichs ist noch im Nordostprofil in Form eines flachen, mit einer Steinplatte gedeckten Kanals erkennbar, der durch die Verlegung von Rohr 1 gestört wurde (Abb. 9). Die gedachte Verlängerung von Rohr 3 hingegen trifft exakt auf die Öffnung im östlichsten Tabernakel der Brunnenrückwand.

${ }_{17}$ Die Unterkante von SE 35 konnte aufgrund der vielen, in unterschiedliche Richtung verlaufenden Rohre nicht erreicht werden.

${ }^{18}$ Mü 2005/48 ist leider nicht bestimmbar, s. u. zu den Fundmünzen Kapitel 4 (M. Pfisterer). 


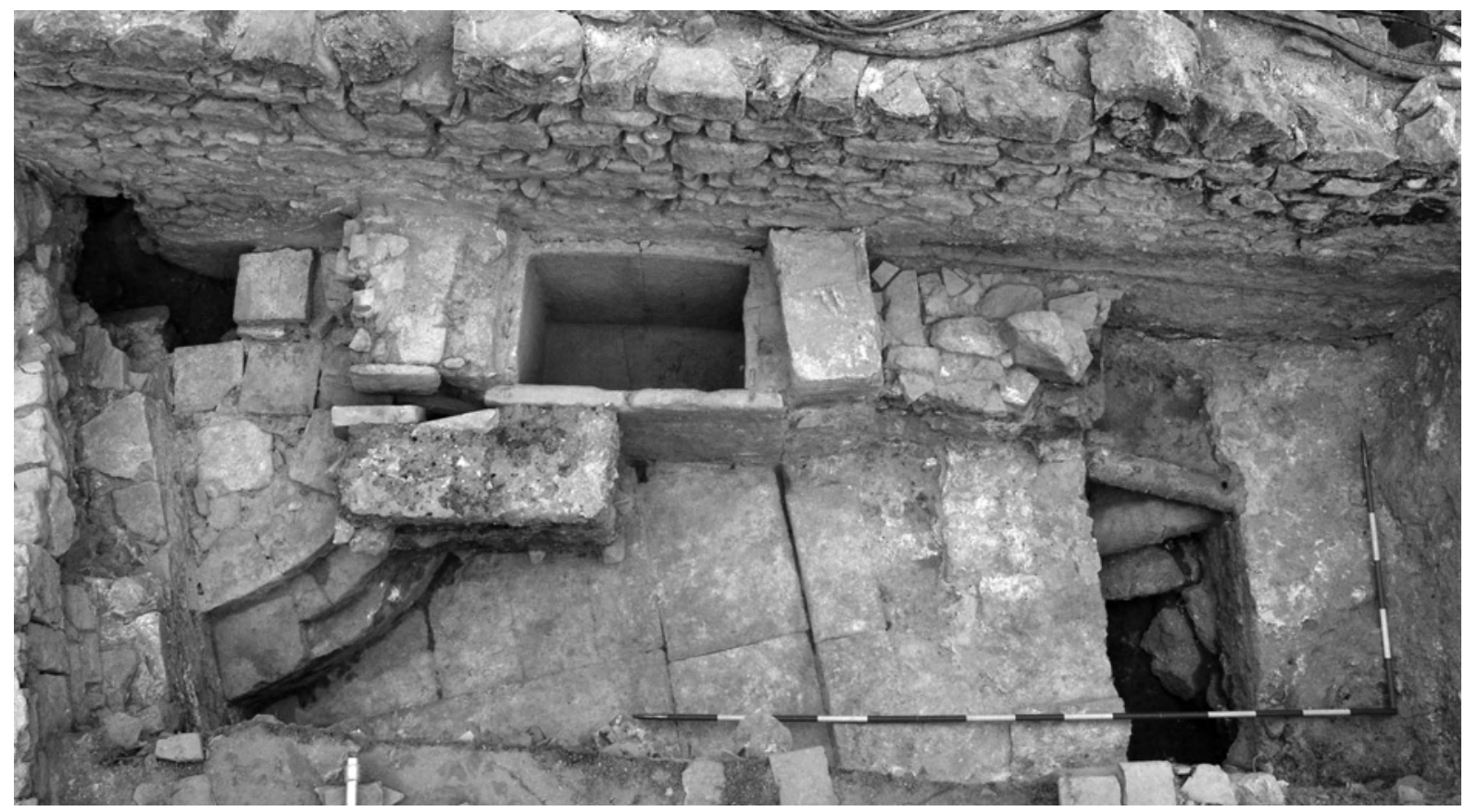

5 Das kaiserzeitliche Wasserverteilungssystem hinter der Rückwand des Nymphaeum Traiani

Der Boden aus fein gefügten Marmorplatten, der den Ostteil der Sondage nach unten hin abschließt, stellt mit hoher Wahrscheinlichkeit das kaiserzeitliche Nutzungsniveau des Areals hinter dem Nymphäum dar. Eine leichte Stufe als Westabschluss des Plattenbodens lässt vermuten, dass es sich dabei um einen Hofbereich (Peristyl?) gehandelt haben könnte. $\mathrm{Zu}$ den einen solchen Hof umgebenden Gängen könnte ein Mosaikboden gehört haben, von dem geringe Reste im Südwestteil der Sondage angetroffen wurden. Dieser Teil der kaiserzeitlichen Bebauung dürfte bei einer Reparatur der Tonrohre in severischer Zeit gestört und nicht wiederhergestellt worden sein. Als Gehniveau errichtete man vielmehr einen kompakten, mehrschichtigen Estrich (SE 33) von 8-12 cm Stärke. Dieser bestand aus grobem Untermörtel, geglätteter Zwischenschicht und einer abschließenden Mörtellage mit groben Ziegelbruchstücken und Marmorkorn. Holzkohlekomponenten sind in allen Lagen zu finden. Die oberste, ca. $2 \mathrm{~mm}$ dicke Schicht ist wohl aufgrund seiner hydraulischen Eigenschaften äußerst reich an Ziegelsplitt ${ }^{19}$.

Zusammenfassend kann festgehalten werden, dass der Marmorplattenboden - gemeinsam mit den möglicherweise zugehörenden weißen Mosaikresten - die älteste, in die römische Kaiserzeit einzuordnende

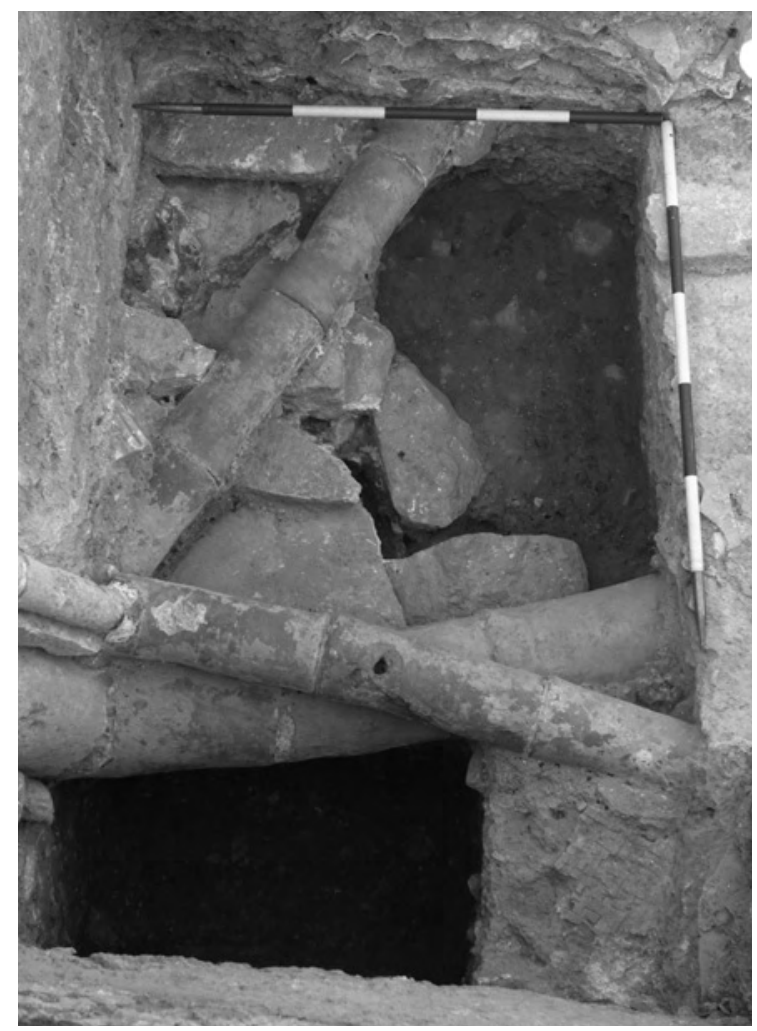

6 Nymphaeum Traiani, Tonrohrleitungen im Westteil der Sondage

\footnotetext{
${ }^{19}$ Für diese Analyse sei W. Prochaska sehr herzlich gedankt.
} 
Baumaßnahme hinter dem Nymphaeum Traiani darstellt ${ }^{20}$. Die auf dem Marmorboden aufliegende Ziegelsetzung dürfte zu einem späteren Zeitpunkt als Stufen zur Verbindung mit den umliegenden Baustrukturen gedient haben. Die Nutzung dieses Hofareals in Zusammenhang mit entsprechenden, bislang nicht bekannten architektonischen Strukturen erfolgte bis in die zweite Hälfte des 4. Jahrhunderts n. Chr. Danach wurde dieser Bereich nicht mehr für den ursprünglich intendierten Zweck genutzt, wie aus der Einbringung der Brandschuttschicht SE 34 im östlichen Teil der Sondage und der flächigen, kompakten Sinterschicht SE 22 im westlichen Bereich ersichtlich ist.

\subsection{Nutzung des Areals: Handwerkliche und bauliche Aktivitäten}

In weiterer Folge kam es zu einer handwerklichen Aktivität im Bereich nordöstlich des Nymphäums, die sich in zahlreichen Nutzungshorizonten und Aufschüttungen manifestiert.

\begin{tabular}{|c|c|c|c|c|}
\hline SE & FK & Kurzcharakterisierung & Datierung & Querverweise \\
\hline 22 & 36,108 & $\begin{array}{l}\text { Flächige Sinterablagerung im Westteil des Schnittes } \\
\text { mit Sinterbrocken und versinterter Keramik, unter- } \\
\text { halb der Kalklöschgrube }\end{array}$ & $\begin{array}{l}\text { 2. Hälfte } 4 . \text { Jh. oder } \\
\text { frühes } 5 . \mathrm{Jh} . \mathrm{n} \text {. Chr. }\end{array}$ & Mü 2005/24 \\
\hline 32 & $52,105,106$ & $\begin{array}{l}\text { Kompakter, mit Ziegeln und Holzkohle vermischter } \\
\text { Brandschutt, flächig im Ostteil des Schnittes, un- } \\
\text { mittelbar auf dem Plattenboden }\end{array}$ & Ende 4. Jh. n. Chr. & Mü 2005/46 \\
\hline 44 & 102 & $\begin{array}{l}\text { Abfallgrube mit lockerer Verfüllung, die Ziegel- } \\
\text { fragmente und Marmorplatten enthielt }\end{array}$ & - & - \\
\hline 17 & $30,40,44$ & $\begin{array}{l}\text { Kalklöschgrube: feste Schicht mit Kalkbrocken und } \\
\text { gelöschtem Kalk, Ziegelfragmenten und einer An- } \\
\text { zahl großer, eckiger Steine }\end{array}$ & $\begin{array}{l}\text { 4./beginnendes } 5 . \text { Jh. } \\
\text { n. Chr. }\end{array}$ & Mü 2005/20, /21, /22 \\
\hline 16 & & Verfüllung der Kalklöschgrube & - & S 9, S 10 \\
\hline 20 & $34,41,61,100$ & $\begin{array}{l}\text { Nutzungshorizont und Verfüllung der Kalklösch- } \\
\text { grube mit größere Steinen, Kalkbrocken und Resten } \\
\text { von Löschkalk; von Osten nach Westen stark ab- } \\
\text { fallend }\end{array}$ & 4. Jh. n. Chr. & S 11 \\
\hline 26 & $43,81,82,107$ & $\begin{array}{l}\text { Kalklöschgrube mit Resten von Löschkalk, Ziegel- } \\
\text { bruch und Steinen }\end{array}$ & 2. Hälfte 4. Jh. n. Chr. & Mü 2005/25, /39 \\
\hline 28 & 47,101 & $\begin{array}{l}\text { Nutzungshorizont der Kalklöschgrube: harte, teils } \\
\text { verziegelte Schicht mit Ziegelbruchstücken, Bruch- } \\
\text { steinen und Mörtel }\end{array}$ & $\begin{array}{l}\text { Ende 4./Anfang 5. Jh. } \\
\text { n. Chr. }\end{array}$ & - \\
\hline 31 & $50,103,104$ & $\begin{array}{l}\text { Nutzungshorizont der Kalklöschgrube: kompakter } \\
\text { Kies, mit Mörtel vermischt }\end{array}$ & $\begin{array}{l}\text { ausgehendes 3.-- } \\
\text { Anfang 5. Jh. n. Chr. }\end{array}$ & - \\
\hline 21 & 35 & $\begin{array}{l}\text { Abfallgrube (?), die sekundär in die Kalklöschgrube } \\
\text { eingetieft wurde: lockere Verfüllung mit Keramik, } \\
\text { Knochen, Eisen, Steinen, Mörtel- und Ziegel- } \\
\text { brocken }\end{array}$ & 5. Jh. n. Chr. & - \\
\hline
\end{tabular}

Im späten 4. oder frühen 5. Jahrhundert endete die Nutzung des Bereichs hinter dem Nymphaeum Traiani als Hofareal (Abb. 9. 10). Dies zeigt sich im Westteil der Sondage durch eine flächige, kompakte Sinterschicht, die neben größeren Sinterbrocken auch einige Fragmente stark versinterter Keramik enthielt. Die massive Ablagerung weist darauf hin, dass zu einem gewissen Zeitpunkt die Tonrohre undicht wurden und hier über einen längeren Zeitraum, wahrscheinlich mehrere Jahre hindurch ${ }^{21}$, immer wieder Wasser austrat. Dies trifft auch auf die dicke Versinterung der Fallrohre zu. SE 22 kann aufgrund der Keramik in die zweite Hälfte des 4. bzw. das frühe 5. Jahrhundert n. Chr. datiert werden, auch Mü 2005/24, eine Maiorina von Constantius II (351-354), liefert einen terminus post quem.

Im Ostteil des Areals, über dem Marmorplattenboden, wurde ein höchstwahrscheinlich umgelagerter,

${ }^{20}$ Die Marmorplatten des Bodens wurden nicht gehoben, weshalb eine nähere zeitliche Einordnung leider nicht möglich ist.

${ }^{21}$ Für diesen Hinweis danke ich wiederum W. Prochaska. 


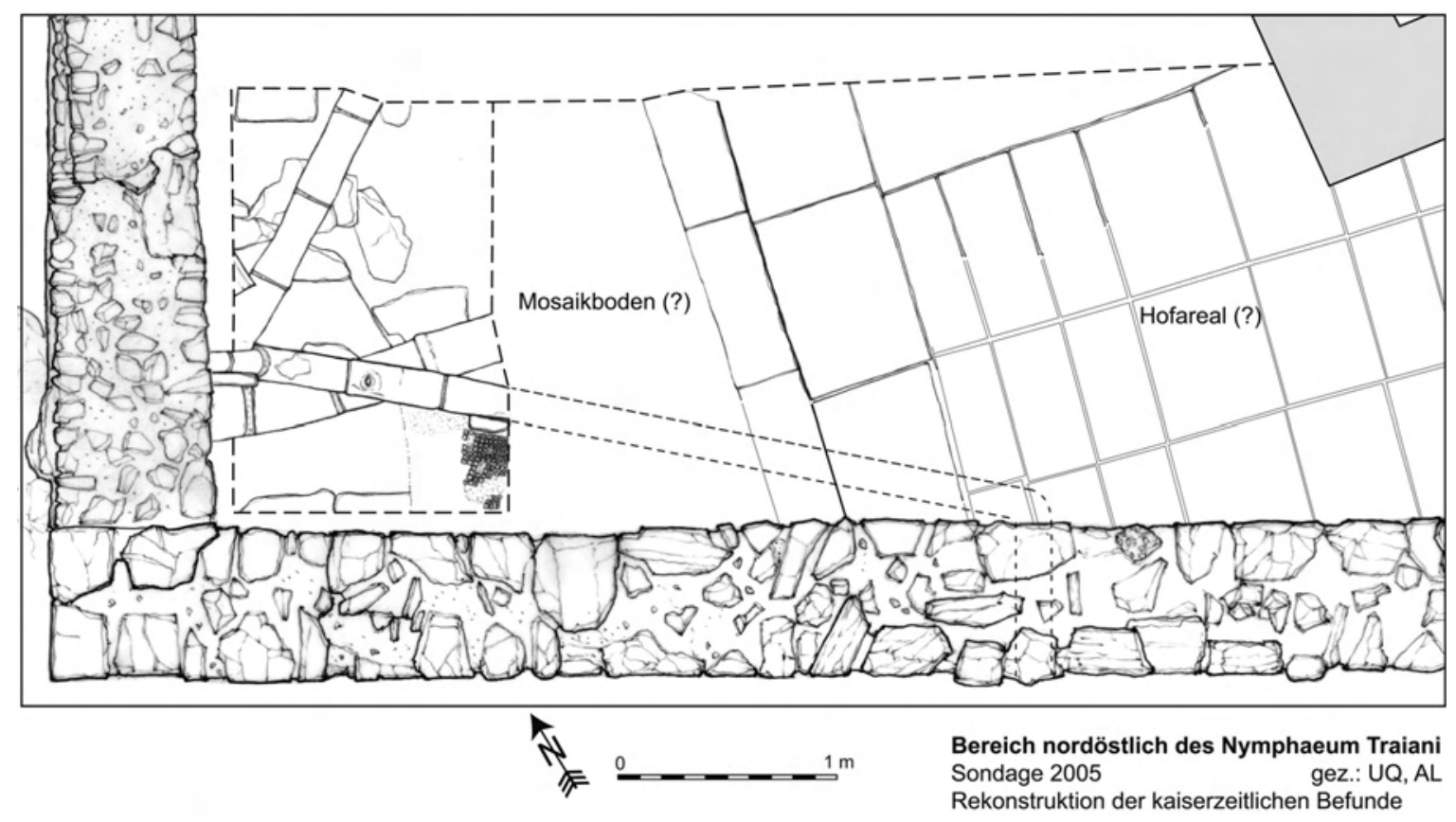

7 Nymphaeum Traiani, Tonrohrleitungen und Rekonstruktion der kaiserzeitlichen Befunde

kompakter, mit Ziegeln und Holzkohle gemischter Brandschutt über dem ehemaligen Hofpflaster eingebracht (SE 32 mit der darin eingetiefter Abfallgrube SE 44). Diese mehr als $30 \mathrm{~cm}$ starke Schicht kann an das Ende des 4. oder den Anfang des 5. Jahrhunderts n. Chr. datiert werden. Es handelt sich möglicherweise um umgelagerten Werkstattschutt ${ }^{22}$. Bevor der Schutt eingefüllt wurde, setzte man noch ein Marmorbecken auf das ehemalige Hofpflaster und schuf eine Unterkonstruktion für eine neue (Blei?-)Leitung, die Wasser vom Nymphäumszufluss in das Steinbecken leitete. Dieses wurde von einer noch in Resten erhaltenen Steinsetzung eingefasst und durch eine Ziegelsetzung unmittelbar vor dem Becken in Position gehalten (Abb. 11). Aus dieser Nutzungsphase stammt möglicherweise auch die flächige Sinterablagerung SE 22.

In weiterer Folge - noch in der zweiten Hälfte des 4. oder im frühen 5. Jahrhundert - wurde das Areal offenbar für Bauaktivitäten genutzt: In die Planierschicht wurde im Westteil der Sondage zwischen der Brunnenrückwand und dem Zufluss eine Grube eingetieft. Nachdem die Ränder grob mit Fragmenten von Marmorplatten verstärkt worden waren, verwendete man die Vertiefung zum Löschen bzw. `Einsumpfen von Kalk ${ }^{23}$. Diese Löschgrube wurde innerhalb kurzer Zeit offenbar mehrfach benutzt: Sie zeigt insgesamt drei farblich voneinander abgesetzte, ca. $10 \mathrm{~cm}$ starke Schichten (SE 17, 20 und 26), denen ebenso viele Nutzungsniveaus (SE 20, 28 und 31) zuzuweisen sind, wobei SE 20 im gesamten Bereich zu verfolgen ist. SE 17 stellt den obersten Abschluss der Kalklöschgrube dar, SE 31 den Arbeitsuntergrund in Form eines festen Kies-Mörtel-Gemisches. Alle diese Straten dürften aufgrund des praktischen Arbeitsablaufs in kurzer zeitlicher Abfolge entstanden sein, worauf auch die Ergebnisse der Keramikauswertung schließen lassen. Die Nutzung der Grube zum Kalklöschen, ein Vorgang, für den Wasser benötigt wird, steht in engem Zusammenhang mit dem steinernen Becken und der Zuleitung zum Nymphaeum Traiani: Gebrannter Stückkalk ist nur beschränkt lagerfähig und verliert für die Verwendung als Mörtel an Festigkeit. Deshalb wird er gelöscht und in eigenen Gruben >eingesumpftı, um zu einem späteren Zeitpunkt als Baumörtel oder Kalkverputz bzw. -anstrich verwendet werden zu können ${ }^{24}$. Aus der Verfüllung der Kalklöschgrube stammen auch Skulpturenfragmente (S 9-11), die Abfallprodukte des dem `Einsumpfen des Kalkbrennens sein könnten.

\footnotetext{
22 s. u. Kapitel 3.2 (A. Waldner).

${ }^{23}$ Für die Bestimmung der Proben aus diesem Bereich sei wieder W. Prochaska sehr herzlich gedankt.

24 Filgis - Blum - Aslan 2005, 235-238; s. auch Dix 1982, 337-339 mit Angaben zu literarischen und bildlichen Quellen.
} 


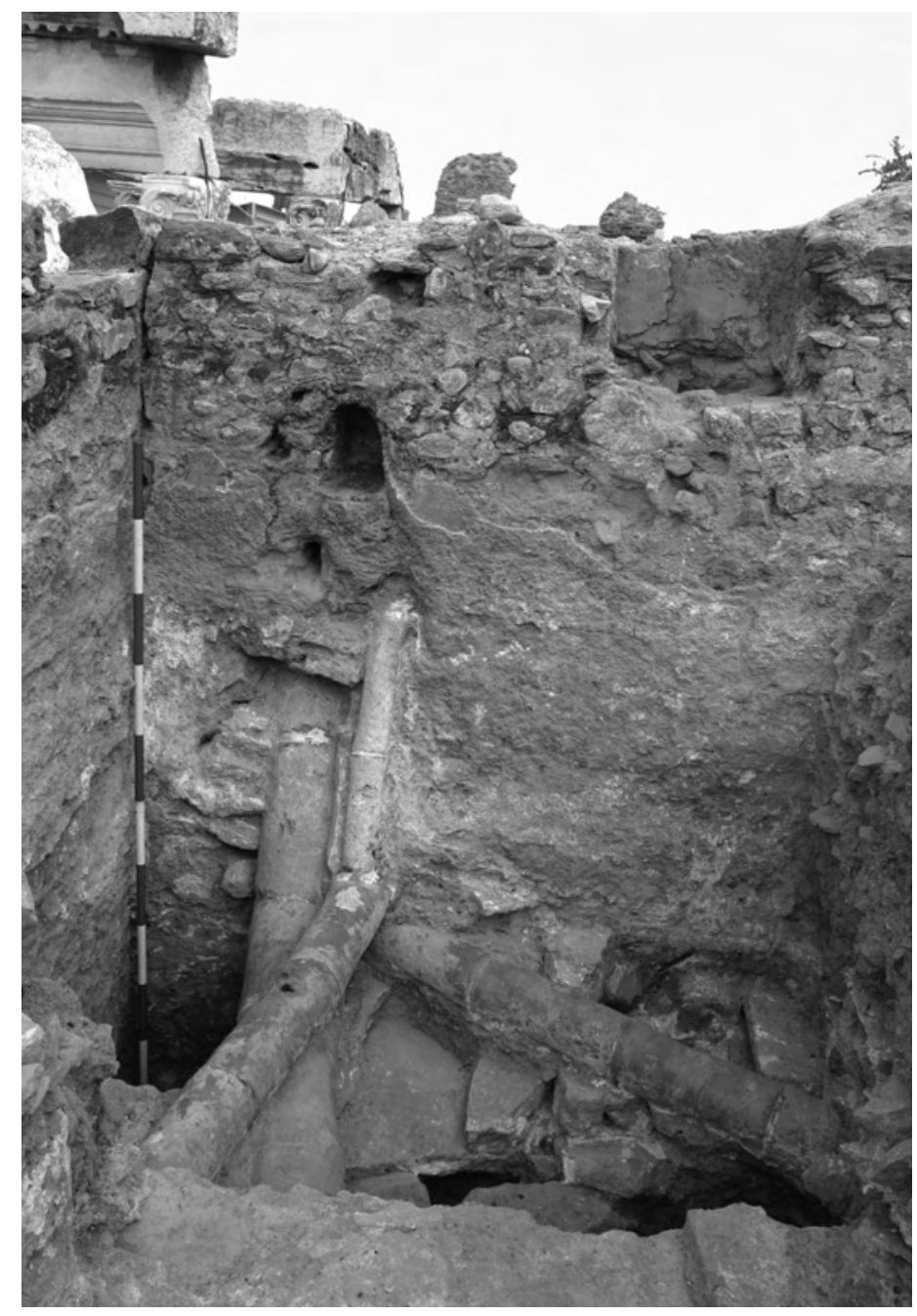

8 Die Fallrohre an der Ostseite des Zuflusses zum Nymphaeum Traiani

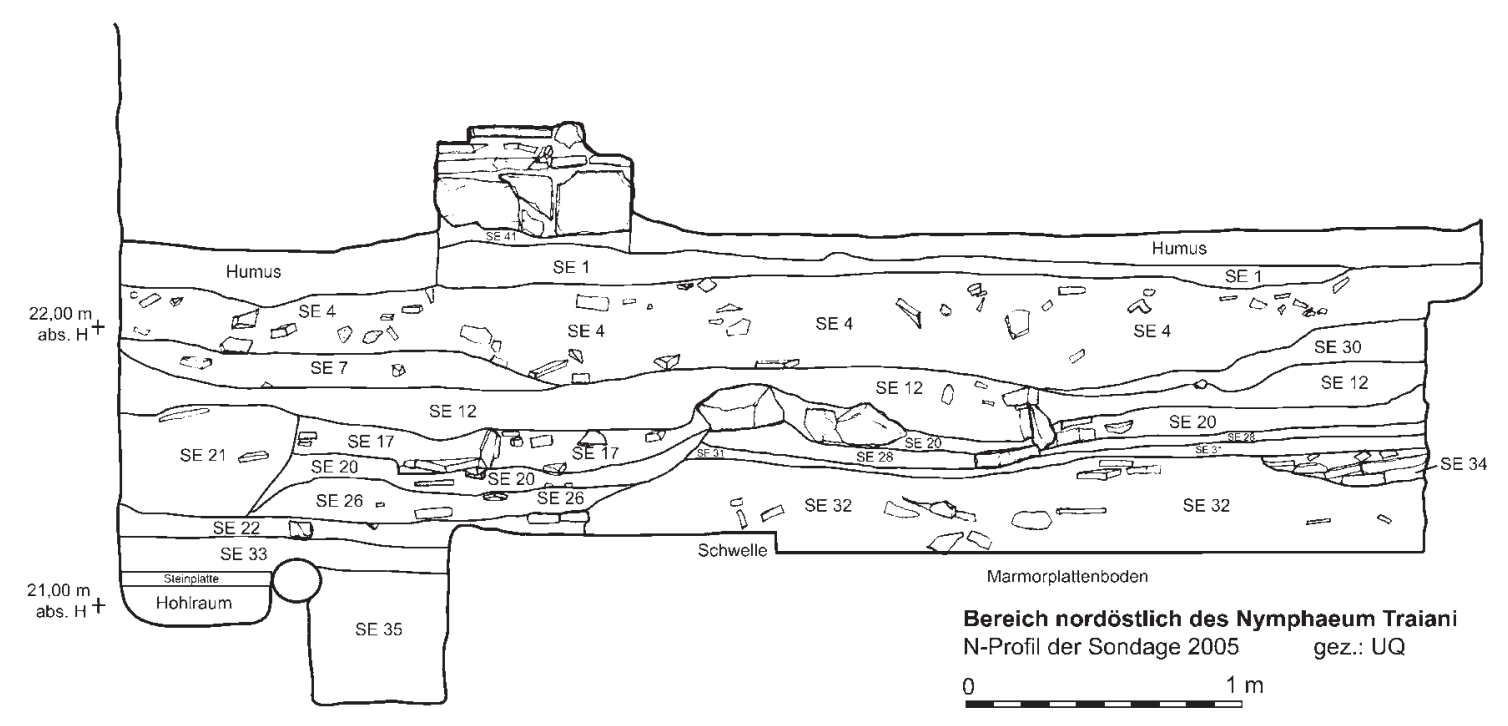

9 Nymphaeum Traiani, Nordprofil der Sondage 
Nach der Aufgabe der Kalklöschgrube wurde wiederum eine Schuttplanierung aufgebracht (SE 12, Abb. 9. 12), die den tiefer liegenden Bereich versiegelt und sich flächig über den gesamten Schnitt mit Ausnahme der Bereiche des Steinbeckens und seinem Zufluss erstreckte (s. u.). Die zeitliche Einordnung in das 5. Jahrhundert n. Chr. liefert einen terminus ante quem für die Aufgabe der Kalklöschgrube sowie die darin eingetiefte Abfallgrube SE 21. Das Steinbecken und seine Zuleitung hingegen bleiben frei und wurden offenbar auch danach weiter genutzt.

\subsection{Spätantike Verbauung}

\begin{tabular}{|c|c|c|c|c|}
\hline SE & FK & Kurzcharakterisierung & Datierung & Querverweise \\
\hline 12 & $19,27,80,98,99$ & $\begin{array}{l}\text { Eingefüllter Brandschutt: inhomogene Schicht mit } \\
\text { Ziegelschutt, Mörtelbröckchen, Holzkohle, Keramik } \\
\text { und kleinen Steinen }\end{array}$ & $\begin{array}{l}\text { 1. Hälfte 5. Jh. n. } \\
\text { Chr. }\end{array}$ & $\begin{array}{l}\text { Mü 2005/13, /14, } \\
/ 15, / 35, / 36 \mathrm{~A} \text { und B }\end{array}$ \\
\hline 14 & 22 & $\begin{array}{l}\text { Ausriss der ehemals vorhandenen Bleileitung (?): } \\
\text { lockere, dunkelbraune Erde mit Holzkohle und } \\
\text { Keramik }\end{array}$ & - & - \\
\hline 8 & $10,14,91,92$ & $\begin{array}{l}\text { Verfüllung des Wasserbeckens mit lockerer, hell- } \\
\text { brauner Erde, die Ziegelbrocken, Holzkohle, Kera- } \\
\text { mik, Glas und Knochen enthielt }\end{array}$ & 6. Jh. n. Chr. & Mü 2005/4 \\
\hline 34 & 57,67 & $\begin{array}{l}\text { Verfüllung des ausgerissenen Rohres im Anschluss } \\
\text { östlich an das Wasserbecken }\end{array}$ & $\begin{array}{l}\text { ausgehendes } \\
5 . / 1 \text {. Hälfte } 6 . \text { Jh. }\end{array}$ & S 8 \\
\hline 11 & $18,26,79$ & $\begin{array}{l}\text { Abfallgrube mit sehr dunkler, lockerer Verfüllung, } \\
\text { die viel Keramik enthielt }\end{array}$ & - & Mü 2005/10,/34 \\
\hline 10 & $12,21,25,27,78$ & $\begin{array}{l}\text { Aufschüttung bzw. Abfallgrube: sehr harte, kom- } \\
\text { pakte Schicht, die neben Steinen und Ziegelfrag- } \\
\text { menten mehrere Skulpturenfragmente enthielt, } \\
\text { darunter den Kopf einer Kopie des Doryphoros }\end{array}$ & $\begin{array}{l}\text { Ende 6./ Anfang } \\
\text { 7. Jh. n. Chr. }\end{array}$ & $\begin{array}{l}\text { S 1-6, Mü 2005/5, } \\
/ 6, / 8\end{array}$ \\
\hline 7 & $9,17,77,96$ & $\begin{array}{l}\text { Aufschüttung, flächig im nördlichen Teil des ge- } \\
\text { samten Sondagenbereichs }\end{array}$ & spätes 6. Jh. & Mü 2005/9 \\
\hline 9 & 11 & Aufschüttung & spätes 6. Jh. & - \\
\hline 30 & $49,51,53,54,97$ & $\begin{array}{l}\text { Inhomogene Aufschüttung im Nordostteil des } \\
\text { Schnittes }\end{array}$ & $\begin{array}{l}\text { ausgehendes } 6 . / \text { frü- } \\
\text { hes } 7 . \text { Jh. n. Chr. }\end{array}$ & S 7 \\
\hline 4 & $6,16,75,94$ & $\begin{array}{l}\text { Aufschüttung, mit auffallend vielen Marmorplatten- } \\
\text { fragmenten, flächig fast im gesamten Bereich }\end{array}$ & $\begin{array}{l}\text { ausgehendes } 6 . / \text { frü- } \\
\text { hes } 7 . \mathrm{Jh} \text {. }\end{array}$ & - \\
\hline 1 & $2,13,74,90$ & $\begin{array}{l}\text { Hellgraue, mörtelige Schicht, vermutlich Unter- } \\
\text { konstruktion eines Plattenbodens, flächig fast im } \\
\text { gesamten Schnitt }\end{array}$ & $\begin{array}{l}\text { 1. Hälfte 7. Jh. n. } \\
\text { Chr. }\end{array}$ & Mü 2005/31 \\
\hline 41 & 73 & $\begin{array}{l}\text { Dunkelbraune, inhomogene Schicht unterhalb der } \\
\text { Mauer M } 3\end{array}$ & - & Mü 2005/30 \\
\hline 2 & $3,46,86,87$ & $\begin{array}{l}\text { Oberster Abschluss über dem Wasserbecken: locke- } \\
\text { re, hellbraune Erde mit Ziegelbruch, kleinen Stei- } \\
\text { nen und Keramik }\end{array}$ & $\begin{array}{l}\text { 2. Hälfte 7. Jh. n. } \\
\text { Chr. }\end{array}$ & - \\
\hline
\end{tabular}

Nach der Aufgabe der Kalklöschgrube wurde nahezu flächig im gesamten Schnitt eine ca. $20 \mathrm{~cm}$ starke, mit Holzkohle durchsetzte Planierschicht eingebracht (SE 12, Abb. 9. 12), die in das 5. Jahrhundert n. Chr. datiert werden kann. Nach Auswertung des Fundmaterials dürfte es sich um Werkstattschutt - möglicherweise aus dem Bereich hinter dem Nymphaeum Traiani - handeln ${ }^{25}$. Das Steinbecken und seine Zuleitung blieben auch in der Spätantike von den Aufschüttungen in diesem Bereich ausgenommen; dies zeigt, dass das Nymphaeum Traiani noch als Brunnen bestand, zumal seine Wasserzuleitung offenbar noch intakt war und Wasser entnommen werden konnte. Die östliche Verklammerung des Steinbeckens hinter seiner Rück-

\footnotetext{
${ }^{25}$ s. u. Kapitel 3.3 (A. Waldner)
} 


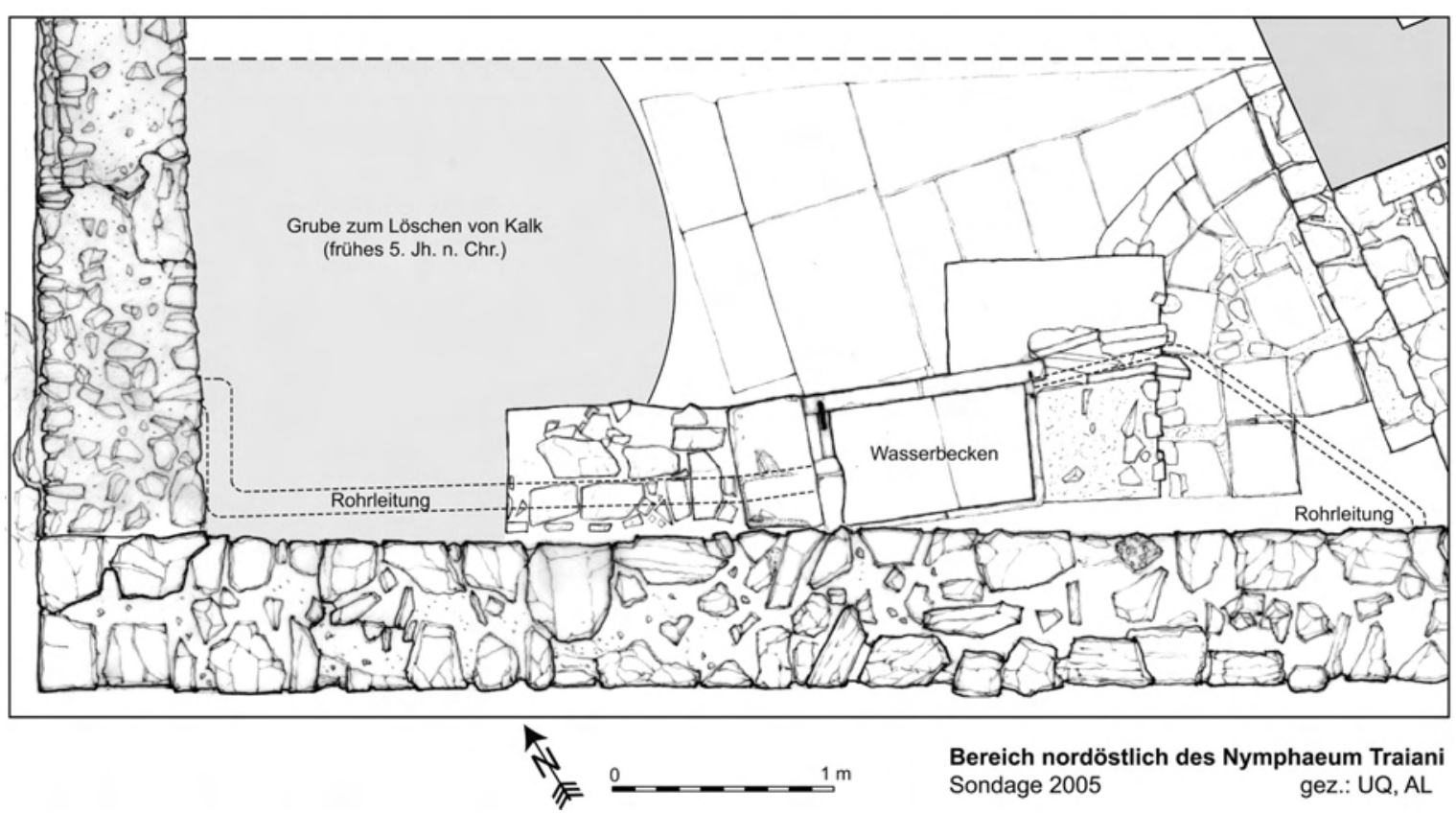

10 Spätantike Befunde und Rekonstruktion von Rohrleitungen im Bereich der Rückwand des Nymphaeum Traiani

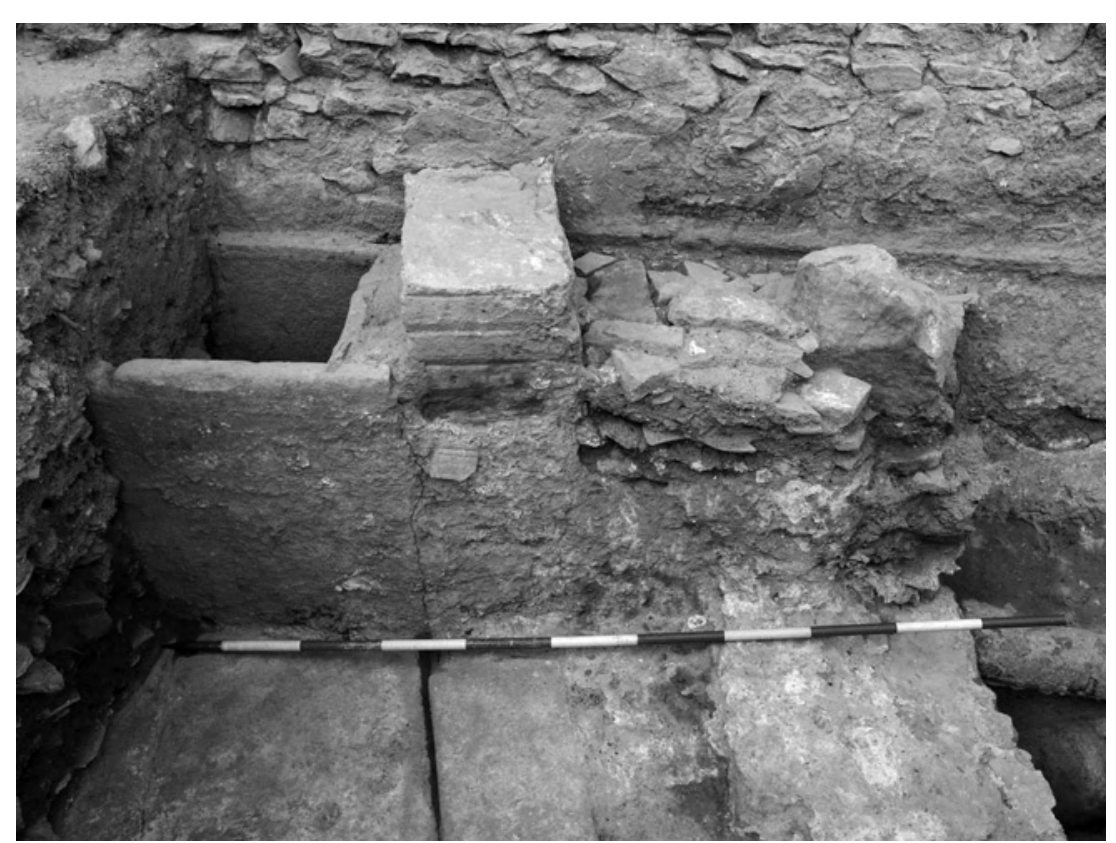

11 Nymphaeum Traiani, Wasserbecken und Bettung für eine Rohrleitung

wand wurde in der Spätantike durchschlagen und in der Planierschicht (SE 12) ein weiteres Rohr verlegt. Dieses führte $\mathrm{zu}$ einer sekundären Öffnung in der Rückwand der Fassade des Nymphäums im Bereich des östlichen Seitenflügels. $\mathrm{Ob}$ es sich um eine weitere Wasserableitung für Bauaktivitäten handelt, oder ob diese Leitung in $\mathrm{Zu}$ sammenhang mit der Wasserführung innerhalb der Brunnenfassade steht, lässt sich aus dem Befund nicht mehr erschließen.

Die Wasserinstallationen hinter der Brunnenrückwand wurden in weiterer Folge aufgegeben. Dies dürfte im 6. Jahrhundert erfolgt sein, wie die Verfüllung des Beckens (SE 8) nahelegt. Auch die oben angesprochene Weiterleitung des Wassers in den Ostflügel muss spätestens zu diesem Zeitpunkt, möglicherweise auch früher (ausgehendes 5. Jh./1. Hälfte 6. Jh.) aufgegeben, die Leitung ausgerissen und die Ausrissgrube verfüllt worden sein (SE 34). Darüber wurden wiederum mehrere Aufschüttungen bzw. Abfallgruben aufgebracht (SE 11). Von besonderem Interesse ist das unmittelbar über dem Ausriss der Bleileitung (SE 14) befindliche, ca. $20 \mathrm{~cm}$ starke Stratum (SE 10), das aufgrund der Funde an das Ende des 6./Anfang des 7. Jahrhunderts datiert werden kann. Es enthielt mehrere Skulpturenfragmente (S 1-6), darunter das Kopffragment einer Kopie des Doryphoros aus antonini- 


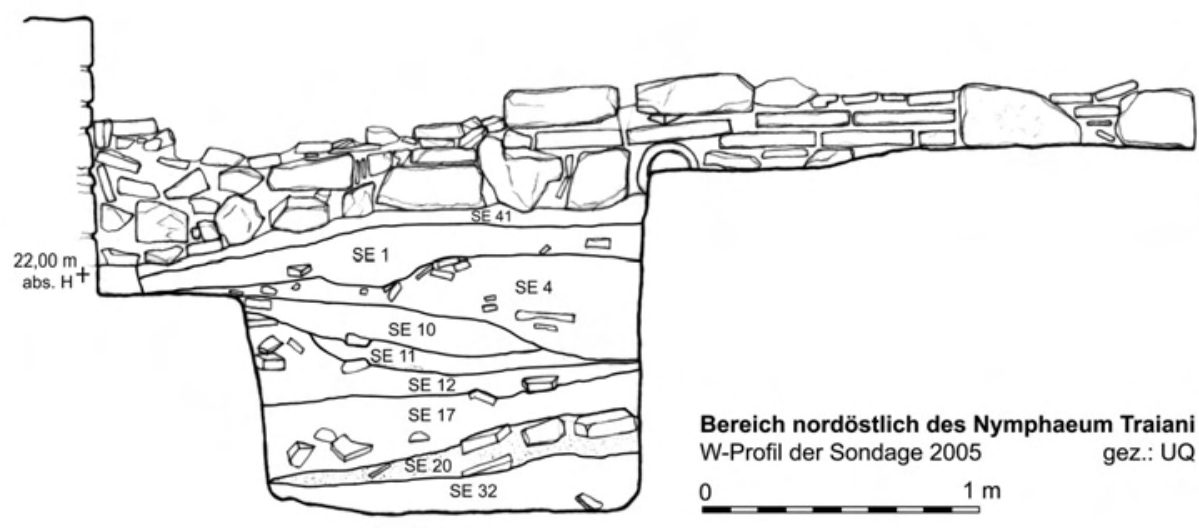

12 Nymphaeum Traiani, Westprofil unterhalb der später abgetragenen Mauer M 3

scher Zeit ${ }^{26}$. Weder die Befundsituation noch Thema und Datierung legen allerdings eine Zugehörigkeit zum Nymphaeum Traiani nahe. Die Keramikauswertung lässt vielmehr vermuten, dass es sich um umgelagerten Werkstattschutt gehandelt haben könnte. In diesem Zusammenhang können die Skulpturenfragmente möglicherweise als Überreste oder Abfall einer Kalkbrennerei gewertet werden. Die flächig in großen Teilen des Schnitts zu verfolgenden Aufschüttungen SE 7 und SE 9 (in der Südwestecke der Sondage) sind in das späte 6. Jahrhundert, SE 30 in das ausgehende 6. oder frühe 7. Jahrhundert zu datieren. Aus dieser Einordnung von SE 30 ergibt sich auch für SE 4 das ausgehende 6. oder frühe 7. Jahrhundert n. Chr. als terminus post quem (Abb. 9). Den obersten Abschluss dieser Niveauerhöhungen bildet die hellgraue, mörtelige, 10-15 cm starke Schicht (SE 1) aus der ersten Hälfte des 7. Jahrhunderts, die höchstwahrscheinlich den Untergrund für einen Plattenboden bildete. Sie ist nur im östlichen Teil durch SE 2 gestört und war sonst wohl ursprünglich im gesamten Bereich flächig anzutreffen. Das im Nordostprofil erkennbare Fehlen von SE 1 westlich der Mauer M 3 dürfte auf die Grabungsaktivitäten F. Miltners zurückgehen.

Die auf einer dünnen Schicht (SE 41) aufsitzende Mauer M 3 stellt die letzte Bauaktivität im Bereich hinter der Rückwand des Nyphäums dar. Für sie kann aufgrund von SE 1 das frühe 7. Jahrhundert n. Chr. als terminus post quem gelten ${ }^{27}$. Die Münzen Mü 2005/2 und Mü 2005/3 aus der Zeit des Heraclius ${ }^{28}$, die beim Putzen der Mauer gefunden wurden, weisen ebenfalls in diese Zeit, auch wenn ihre kontextuelle Aussagekraft stark eingeschränkt ist. Die allerletzte nachweisbare Aktivität stellt das in der Südostecke der Sondage angetroffene Stratum SE 2 dar, welches in die zweite Hälfte des 7. Jahrhunderts n. Chr. datiert werden kann ${ }^{29}$.

U. Quatember

\footnotetext{
${ }^{26}$ s. u. Kapitel 5 (M. Aurenhammer)

27 SE 41 ist nur unterhalb der Mauer erhalten und wurde höchstwahrscheinlich ebenso bei den Grabungsaktivitäten F. Miltners abgetragen wie SE 1 westlich von M 3 .

${ }^{28}$ Mü 2005/2: Heraclius; 40 Num; 613; Con 7h ; 10,76 g; Av.: Leg.?; zwei Kaiser frontal stehend; Rv.: M; 1. A/N/N/O, oben Chrismon, r. Jz., unten $\Delta$, i. A. CONB; MIB 160b. - Mü 2005/3: Heraclius: 40 Num; 614/615; Ms.?; 1 ; 11,65 g: Av.: Leg.?; zwei Kaiser frontal stehend; Rv.: M; r. Jz.; Details schwer lesbar; MIB 160a; doppelte Überprägung: 1. auf älteren 40er im Consulartyp (Phocas?) (Av./Av.); 2. auf älteren 40er CON (Av./Rv.). (Bestimmung M. Pfisterer).

${ }^{29}$ s. u. Kapitel 3 (A. Waldner).
} 


\section{Die Fundkomplexe}

Aus der Grabung nördlich des Nymphaeum Traiani wurden rund 5000 Einzelfunde statistisch erfasst, 640 diagnostische Fragmente wurden zeichnerisch dokumentiert ${ }^{30}$. Es handelt sich dabei meist um kleinteilig gebrochene Fragmente von Keramikgefäßen, die oftmals sekundär beeinträchtigt (z. B. versintert) waren. Das Keramikspektrum umfasst feinkeramische Waren der Kaiserzeit wie Eastern Sigillata B (ESB) und Eastern Sigillata C (ESC), vor allem aber handelt es sich um spätantik-frühbyzantinisches Tafelgeschirr, wobei Gefäße der Gattungen Late Roman C (LRC), Ephesian Red Slip Ware (ERSW), African Red Slip Ware (ARS) sowie >Mäandertalsigillata vertreten sind. Zum Fundspektrum gehören außerdem Amphoren; der Großteil der typologisch bestimmbaren Stücke ist den lokalen spätantiken Typen Peacock - Williams 45/Late Roman Amphora 3 (LRA 3) und Ephesos 56 zuzuordnen. Küchenware und Gebrauchskeramik sowie Lampen, Glasgefäße und verschiedene Kleinfunde aus Metall und Bein runden das Spektrum ab. Zum Inventar zählen außerdem Produktionszeugnisse wie Gusstiegel, ferner Mosaiktesserae, kleine Wandmalereifragmente, Verputzbrocken, Holzkohlefragmente und Tierknochen.

Im Folgenden soll nicht die gesamte Fundevidenz besprochen werden, sondern es erfolgt eine Konzentration auf ausgewählte Funde, die jeweils zur chronologische Einordnung der im Norden des Nymphaeum Traiani angetroffenen Strukturen herangezogen werden können, Rückschlüsse auf die Funktion der Befunde erlauben oder besondere Merkmale aufweisen ${ }^{31}$.

Der Aufbau der Fundbesprechung folgt - gleich jenem der Befund- und Stratigraphiebesprechung ${ }^{32}-$ der Untergliederung in die drei Phasen der Nutzung des Areals nördlich des Nymphaeum Traiani.

\subsection{Fundkomplexe in Zusammenhang mit dem Nymphaeum Traiani (SE 35, SE 33)}

Die Schicht zwischen den Wasserrohren im Westteil von Schnitt 1 (SE 35) enthielt rund 550 keramische Fragmente. Datierend sind zwei ESB-Ränder der Form Atlante $60^{33}$, die allerdings sehr stark versintert sind. Große Teller dieser Form wie Kat. 1 treten in Ephesos bis in die severische Zeit auf ${ }^{34}$. Zwei kleine, flache ESB-Bodenfragmente mit Resten konzentrischer Rillen sind vermutlich derselben Form zuzuordnen wie Kat. 2, sie sind ebenfalls versintert und stark verbrannt. Das gleichfalls sekundär beeinträchtigte Bodenfragment Kat. 3 ist hingegen der Form Atlante 68 oder 58 zuzuweisen $^{35}$ : Es hat einen mittigen sternförmigen Stempel auf der Bodeninnenseite. Das Stück ist in das 1./2. Jahrhundert zu datieren. Von einem ESC-Teller der Form Atlante L26 B haben sich zwei - allerdings nicht anpassende - Fragmente erhalten (wie Kat. 4) ${ }^{36}$. Die Form tritt ab der Mitte des 1. Jahrhunderts bis in die Mitte des 2. Jahrhunderts auf, aufgrund des Erhaltungszustandes ist auf eine mindestens einmalige Umlagerung zu schließen. Ein Schälchen mit breitem Rand und geschwungener Wand kann formtypologisch als ESC-Teller der Form Atlante L9 identifiziert werden $^{37}$ (Kat. 5). Man wird nicht fehlgehen, das versinterte Gefäßfragment, dessen Überzug stark abgerieben ist, in das 1./2. Jahrhundert n. Chr. zu datieren. Erwähnenswert ist außerdem ein großer Teller mit leicht eingebogenem Rand, der weiße und vulkanische Einschlüsse beinhaltet und vermutlich aus Çandarlı stammt (Kat. 6). Er ist der ESC-Form Atlante L6 zuzuweisen, die von der Mitte bis zum Ende des 1. Jahrhunderts n. Chr. auftritt ${ }^{38}$. Außerdem sind fünf Fragmente von Feinwarebechern mit Trichterrand vertreten,

\footnotetext{
${ }^{30}$ Die Finanzierung der Fundbearbeitung erfolgte über das FWF-Projekt Nr. P17617, die der Manuskripterstellung über das ÖAI. Für die zeichnerische Dokumentation im Depot des österreichischen Grabungshauses in Selçuk waren A. Lätzer (2006), J. Struber (2006/2007) und E. Profant (2007) verantwortlich. J. Struber übernahm zusätzlich die Digitalisierung der Zeichnungen. Ihnen sei an dieser Stelle herzlich gedankt. Die Tafeln wurden von der Verf. erstellt.

${ }^{31}$ Die jeweils angeführten Parallelen für die Stücke beziehen sich großteils auf Ephesos.

32 s. o. Kapitel 2 (U. Quatember).

${ }^{33}$ Hayes 1985, 64.

${ }^{34}$ Ladstätter 2000, 100; Ladstätter 2008, 99.

${ }^{35}$ Hayes 1985, 68 (Atlante 68). Zu der häufiger auftretenden Form Atlante 58 s. Hayes 1985, 63.

${ }^{36}$ Vgl. Beyll 1993, Taf. 7, 81.

${ }^{37}$ Hayes 1985, 76.

${ }^{38}$ Hayes $1985,75$.
} 
die im 2. und 3. Jahrhundert n. Chr. charakteristische Bestandteile des Trinkgeschirrs darstellen (wie Kat. 7. 8). In der Wohneinheit 4 des Hanghauses 2 von Ephesos treten sie vermehrt in Fundkomplexen ab der Bauphase II bzw. ab dem frühen 2. Jahrhundert n. Chr. auf ${ }^{39}$. Ein Randfragment einer Amphore vom Typ Peacock - Williams 45 (Kat. 10) ist aufgrund des außen umlaufenden Absatzes als früh- bis mittelkaiserzeitlich einzustufen, ebenso zwei geöffnete Amphorenfüße dieses Typs (Kat. 11. 12) ${ }^{40}$, wobei das flächig versinterte Fragment Kat. 12 sogar noch in das 1. Jahrhundert v. Chr. fallen könnte. Die ersten Vertreter dieser an ihrem bräunlich violetten, stark glimmerhaltigen Fabric leicht zu identifizierenden Amphorengattung erscheinen bereits am Ende des 1. Jahrhunderts v. Chr., der langlebige Typ bleibt aber unter Veränderung seiner Form bis in das 6./7. Jahrhundert verbreitet. Für die Form kann eine chronologisch relevante Entwicklung nachgezeichnet werden, so z. B. weisen frühe Vertreter des Amphorentyps nur einen Henkel (`One Handle Jar $২)^{41}$ auf, der Rand ist außen durch einen Absatz untergliedert. Außerdem schließt sich der Fuß zusehends und wird im 6. Jahrhundert zu einem massiven geschlossenen Knauf. Die späten Amphoren vom Typ Peacock Williams 45 werden auch unter der Bezeichnung Late Roman Amphora 3 (LRA 3) geführt ${ }^{42}$.

Mehrere Lampenfragmente runden das Spektrum ab, es handelt sich u. a. um einen Lampenboden, ein Schulterfragment und ein Wandfragment von Lampen des Typs Red-on-white (ROW, Kat. 13. 14), die ab tiberischer Zeit in der Region um Ephesos produziert werden und bis in das 3. Jahrhundert verbreitet sind ${ }^{43}$.

Aufgrund der Keramikfunde kann SE 35 in das 2. Jahrhundert n. Chr. bis maximal in die severische Zeit datiert werden. An das Ende des 2. Jahrhunderts verweist hingegen eine der beiden Münzen aus SE 35: Sie ist Iulia Domna (193-217 n. Chr.) zuzuweisen ${ }^{44}$ - demnach kann der Bereich zwischen den Wasserrohren frühestens in severischer Zeit verfüllt worden sein.

Aus dem Estrich SE 33, der im Westteil der Sondage das Gehniveau über den Tonrohrleitungen bildet, stammen bis auf ein kleines Bodenfragment einer Grauen Platte mit schwarzem Überzug ${ }^{45}$ keine chronologisch aussagekräftigen Funde. Platten dieser Ware zählen zu den wichtigsten ephesischen Keramikprodukten des 1. Jahrhunderts v. Chr. und des frühen 1. Jahrhunderts n. Chr. ${ }^{46}$. Ein Randfragment einer Schüssel im Küchenware-Fabric (Kat. 15) macht aufgrund seiner Beschaffenheit einen ebenfalls späthellenistischen Eindruck $^{47}$. Zwei Glasfunde - ein massiver Standring mit außen aufgelegtem dünnem Glasfaden (Kat. 16) ${ }^{48}$ sowie ein Fragment eines Bechers oder Kruges mit zylinderförmig aufsteigendem Hals (Kat. 17) ${ }^{49}$, leicht ausgebogenem Rand und verdickter Lippe - weisen in die frühe bis mittlere Kaiserzeit. In dem Estrich befanden sich außerdem Wandfragmente von Amphoren des Typs Peacock - Williams 45. Bedingt durch die lange Laufzeit dieser Amphorenform können sie nicht näher datiert werden. Der kleinteilige Erhaltungszustand der insgesamt 38 registrierten Einzelfunde ${ }^{50}$ und die frühe Datierung der wenigen bestimmbaren Fragmente lassen darauf schließen, dass es sich um mehrfach umgelagerten Schutt handelt, der für den Aufbau des Estrichs SE 33 verwendet wurde.

Folgerichtig kann für die Datierung des Estrichs nur ein terminus post quem im 2. Jahrhundert $\mathrm{n}$. Chr. (bzw. nachseverisch) angegeben werden, der sich aus der zeitlichen Einordnung der Füllung zwischen den Tonrohrleitungen darunter (SE 35) erschließen lässt.

39 Vgl. z. B. Ladstätter 2005, 239 K 470-473.

40 Vgl. z. B. Ladstätter - Steskal 2008, Nr. 1. 12.

41 Bezeczky 2005, $203 \mathrm{f}$.

42 Zur Entwicklung der Amphoren vom Typ LRA 3 s. Peacock - Williams 1986, 188; Bezeczky 2005, 204; Ladstätter 2007, 212; Ladstätter 2008, 180-182; Ladstätter - Steskal 2009.

${ }^{43} \mathrm{Zu}$ Chronologie und Typologie der ROW-Lampen s. Ladstätter 2005, u. a. 238; Ladstätter $2007,211$.

44 Mü 2005/47, s. die Münzliste in Kapitel 4 (M. Pfisterer).

45 Dieses wurde aufgrund seiner Kleinteiligkeit nicht in den Katalog aufgenommen.

${ }^{46}$ Ladstätter 2003, 24; Rogl 2004, 211-214. Die lokale Produktion Grauer Ware mit schwarzem Überzug ist durch chemische Analysen bezeugt; Zabehlicky-Scheffenegger u. a. 1996, 41-59.

${ }^{47}$ Kennzeichnend für hellenistische Küchenwaren ist der im Vergleich zu den kaiserzeitlichen Kochgefäßen grob gemagerte Ton, der u. a. durch Einschlüsse großer Glimmerplättchen gekennzeichnet ist, die auch an der Gefäßoberfläche sichtbar sind. Vgl. beispielsweise die Küchenware aus der Brunnenfüllung in SR 9C des Hanghauses 1 und deren Charakterisierung: Ladstätter $2003,37$.

48 Vgl. Harter 1999, Taf. 14, 311-313; Formentaf. 2, B 14b.

49 Vgl. Czurda-Ruth 2007, Kat. 387-397; Steskal 2008, Taf. 344, G 11.

50 Darunter auch Mosaiktesserae und Wandverputzbrocken. 
3.2 Fundkomplexe aus der Nutzung des Areals für handwerkliche und bauliche Aktivitäten (SE 22, 32, 44, 17, 20, 26, 28, 31, 21)

Die unmittelbar über dem Estrich SE 33 befindliche Sinterschicht SE 22 gehört offenbar bereits zu den nachnutzungszeitlichen Strukturen nördlich des Nymphaeum Traiani. Sie enthielt zwar insgesamt wenig Fundmaterial, das zudem kleinteilig gebrochen und versintert ist, allerdings tritt darin der Rand eines Kochtopfes bzw. einer Kasserolle auf, der zweifelsohne einer spätantiken Zeitstellung angehört (Kat. 18). Das Gefäß mit breitem ausgebogenem Rand, Deckelfalz und gerippter Wand kann - etwa nach Vergleichsbeispielen aus dem Stadion - in das späte 4. und 5. Jahrhundert datiert werden ${ }^{51}$. Hervorzuheben ist außerdem ein nahezu vollständig erhaltener Gusstiegel (Kat. 19), der von einer Metallverarbeitung zeugen könnte. Ein halb geschlossener Knauf einer Amphore vom Typ LRA 3 (Kat. 20) dürfte ebenfalls in das 4./5. Jahrhundert zu datieren $\operatorname{sein}^{52}$. Chronologisch aussagekräftig ist außerdem ein Lampenfragment (Kat. 21), dessen zentraler, gelochter Griff und die längliche Form des kantigen Lampenkörpers mit auf der Schulter umlaufendem Wulst darauf schließen lassen, dass es sich um eine Doppelschnauzenlampe handelt. Nach Vergleichen, beispielsweise einem Exemplar vom `kleinasiatischen Typ a aus dem Siebenschläfer-Coemeterium ${ }^{53}$ oder aus dem sog. Odeionkanal im Bereich der Basilika am Staatsmarkt ${ }^{54}$, kann die Lampe in das 5. Jahrhundert datiert werden ${ }^{55}$. Unter den Glasfunden ist ein kleines Wandfragment hervorzuheben, das einem Becher der Form Isings 106c (eiförmig) oder Isings 109 (konisch) ${ }^{56}$ zugewiesen werden kann (Kat. 22). Es weist außen Reste eines blauen Tropfendekors auf, wie er auf Glasbechern ab dem 4. Jahrhundert auftritt ${ }^{57}$.

Es ist insgesamt von einer Datierung der SE 22 in die zweite Hälfte des 4. Jahrhunderts bzw. in das frühe 5. Jahrhundert auszugehen. Die einzige Münze aus der Sinterschicht ist Constantius II zuzuweisen und wurde 351-355 n. Chr. geprägt ${ }^{58}$, spricht also nicht gegen die aus dem keramischen Material gewonnene Datierung.

Bei den insgesamt rund 330 registrierten Funden aus SE 32, einer massiven Brandschuttschicht über dem Marmorboden im Osten von Schnitt 1, handelt es sich großteils um keramische Wandfragmente verschiedener Gattungen. Es fanden sich aber auch typologisch bestimmbare, feinchronologisch relevante Stücke, darunter ein ESB-Randfragment, das der Form Atlante 14 zugeordnet werden kann (Kat. 23), welche in die frühe/mittlere Kaiserzeit datiert ${ }^{59}$. Ein weiteres ESB-Wandfragment ist höchstwahrscheinlich der Form Atlante 58 zuzuweisen (Kat. 24), die in der Zeit von $75-125$ n. Chr. produziert wird ${ }^{60}$. Ein Randfragment (Kat. 25) gehört zu einem wohl als bauchig zu rekonstruierenden Feinwarebecher mit Steilrand und eingeschnürter Schulter, wie sie in Ephesos vor allem in claudischen und flavischen Fundkomplexen zu finden sind ${ }^{61}$. Weitere Boden-, Wand- und Henkelfragmente sind dieser ab dem 1. Jahrhundert v. Chr. zu beobachtenden, aber besonders in der Kaiserzeit verbreiteten Gattung zuzuordnen. Ihr Auftreten ist wie das der ESB-Fragmente bemerkenswert, zumal die restlichen Funde wesentlich jünger sind. So handelt es sich bei den zuweisbaren Küchenware-Randfragmenten wie Kat. 26-28 um Kochtopfformen, die in Ephesos bislang erst ab ca. 400 n. Chr. nachgewiesen $\operatorname{sind}^{62}$. Zwei Knauffragmente von LR 3-Amphoren sind halb geöffnet und sind daher als spätkaiserzeitliche Vertreter dieser Amphoren anzusprechen (wie Kat. 29) ${ }^{63}$. Auch sieben Wandfragmente

\footnotetext{
51 Turnovsky 2005a, Abb. 1, 1.

52 Zur Entwicklung des Amphorentyps s. o. Anm. 42.

53 Miltner 1937, Nr. 198. 313.

${ }^{54}$ Mitsopoulos-Leon 2007, 87 L 250.

55 Ein weiteres Vergleichsexemplar aus einer Kanalverfüllung im Bereich der Tetragonos Agora datiert V. Gassner in das 5./6. Jh.: Gassner 1997, 203 Nr. 835 Taf. 65, 91.

56 Isings 1957, 106. 129-130.

57 Vgl. z. B. aus dem Hanghaus 1 in Ephesos: Czurda-Ruth 2007, 103 Kat. 367-368.

58 Mü 2005/24, s. die Münzliste Kapitel 4 (M. Pfisterer).

59 Hayes 1985, 56.

60 Hayes 1985, 63.

${ }^{61}$ Vgl. z. B. Meriç 2002, 75 K 443-450.

62 Vgl. Turnovsky 2005a, Abb. 1, 2.

${ }_{63}$ Vgl. Ladstätter - Steskal 2009, Nr. 13.
} 
von Amphoren des Typs LRA 1 weisen in das spätere 4. Jahrhundert bis in die Mitte des 7. Jahrhunderts ${ }^{64}$. Die Glasfunde - es handelt sich dabei durchweg um kleine Fragmente massiver, angesetzter Standringe von Schalen oder Bechern (Kat. 30-32) - finden in Ephesos beispielsweise im Fundspektrum des Hanghauses 1 Parallelen. Ähnlich gestaltete Böden treten hier vor allem in den durch das Erdbeben im dritten Viertel des 3. Jahrhunderts n. Chr. entstandenen Verschüttungsschichten auf ${ }^{65}$.

Bemerkenswert sind Fragmente von Gusstiegeln in der Brandschuttschicht, die teilweise innen noch Reste einer geschmolzenen Substanz aufwiesen. Bei den Gusstiegeln könnte es sich um Zeugnisse einer Produktion nördlich des Nymphaeum Traiani oder um umgelagerten Werkstattschutt handeln ${ }^{66}$.

Insgesamt ist SE 32 speziell aufgrund der darin auftretenden Küchenware-Formen an das Ende des 4./ Anfang des 5. Jahrhunderts zu datieren. Die einzige Fundmünze aus SE 32 gibt einen terminus post quem in der zweiten Hälfte des 3. Jahrhunderts. Sie ist Gallienus zuzuweisen und wurde 260-268 n. Chr. geprägt ${ }^{67}$. Auffallend ist jedoch das Auftreten älterer ESB- und Feinwareformen, das darauf schließen lässt, dass es sich dabei um umgelagerten Schutt aus dem 1./2. Jahrhundert handelt.

Die Abfallgrube SE 44 enthielt lediglich ein bestimmbares Randfragment einer feinkeramischen Schüssel, das jedoch kleinteilig gebrochen und feinchronologisch nicht aussagekräftig ist.

Ausschlaggebend für die Datierung der Kalklöschgrube (SE 17) ist u. a. ein ARS-Randfragment der Form Hayes 67, die von 360-470 n. Chr. produziert wird (Kat. 33) ${ }^{68}$. Das Stück ist der - nach J. W. Hayes frühen Gruppe 1 zuzuordnen, folglich kann die Datierung zwischen 360 und $420 \mathrm{n}$. Chr. eingegrenzt werden $^{69}$. Eine Amphore mit verdicktem Rand und gerippten Henkeln (Kat. 34) dürfte aufgrund des Fabrics aus dem Schwarzmeergebiet stammen, kann aber keinem bestimmten Amphorentyp zugewiesen werden ${ }^{70}$. Zwei halb geschlossene Amphorenknäufe (Kat. 35. 36) vom Typ LRA 3 deuten hingegen in das 3./4. Jahrhundert ${ }^{71}$. Die in der Grube enthaltenen Küchenwaren sind dem 4./frühen 5. Jahrhundert n. Chr. zuzuordnen. Es handelt sich dabei hauptsächlich um Töpfe/Kasserollen mit innen mehrfach abgestuftem Rand ${ }^{72}$ und um einen

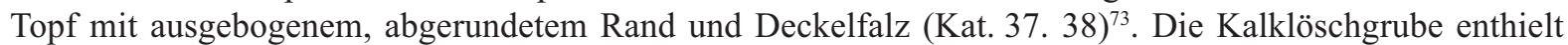
auch einige kleine, tongrundige oder mattrot überzogene Lampenfragmente. Zeitlich sind sie innerhalb der späten Kaiserzeit allerdings nicht näher einzuordnen. Gesondert hinzuweisen ist auf einen Lampenhenkel, dessen Anfertigung nicht sehr sorgfältig erfolgte (Kat. 39). Darauf lässt die intendierte Lochung des Henkels schließen, in welcher der Tonkern haften blieb. Es ist nicht davon auszugehen, dass ein so fehlerhaftes Stück importiert wurde, demnach muss es sich um ein Zeugnis einer lokalen ephesischen Lampenproduktion im 4./beginnenden 5. Jahrhundert handeln. Auch die glimmerhaltige Beschaffenheit des Fragments lässt auf eine Herstellung im lokal-regionalen Umkreis schließen.

Aufgrund der Fundzusammensetzung wird man nicht fehlgehen, die Anlage der Kalklöschgrube in das 4./beginnende 5. Jahrhundert zu setzen. Dies bedeutet allerdings, dass die darin sekundär eingetiefte Abfallgrube SE 21 nur wenig später entstanden sein kann.

Der aus größeren Steinen, Kalkbrocken und Resten von Löschkalk bestehende Nutzungshorizont der Kalkgrube SE 20 enthielt nur wenig feinchronologisch relevantes Fundmaterial, das zudem meist sekundär durch den Löschkalk - stark beeinträchtigt ist. Ein abgebrochener Knauf einer Amphore vom Typ LRA 3 (Kat. 40) kann als beinahe geschlossen rekonstruiert werden und ist demnach in das 4./5. Jahrhundert zu

\footnotetext{
${ }^{64}$ Bezeczky 2005, 205 f.; Peacock - Williams 1986, 187.

${ }_{65}$ Vgl. z. B. Czurda-Ruth 2007, Taf. 28, 576-600.

${ }^{66} \mathrm{Zu}$ Funden von Gusstiegeln im Bereich der Tabernen in der Nordhalle der Kuretenstraße vgl. auch Iro - Schwaiger - Waldner 2009.

${ }^{67}$ Mü 2005/46, s. die Münzliste in Kapitel 4 (M. Pfisterer).

${ }^{68}$ Hayes 1972, 112-116.

${ }^{69}$ Hayes 1972, 116.

${ }^{70}$ T. Bezeczky sei für die Herkunftsbestimmung des Stücks gedankt.

${ }^{71}$ s. o. Anm. 42.

${ }^{72}$ Unter den Funden des Stadions ist ein Auslaufen dieser Topfform in der Mitte des 5. Jhs. n. Chr. zu beobachten. Turnovsky 2005a, Abb. 1, 1; s. auch Ladstätter 2008, Abb. 31, 3. 6. 11.

${ }_{73}$ Diese Topfform tritt zwar vor allem im 5./6. Jh. n. Chr. auf (vgl. z. B. Turnovsky 2005a, Abb. 1, 14-16; Meriç 2002, K783-785), die abgerundete, >weiche` Form des hier vorliegenden Randes erlaubt aber durchweg eine etwas frühere Datierung.
} 
datieren ${ }^{74}$. Ein küchenkeramischer Deckel mit verdicktem Rand (Kat. 41) ist möglicherweise in dieselbe Zeit zu setzen, ebenso fünf gerippte Wandfragmente von Amphoren, die Parallelen im Hanghaus 2 (3. Viertel 3. Jh.) und im Vediusgymnasium ${ }^{75}$ sowie im Schiffswrack von Yassi Ada aus dem 4. Jahrhundert finden ${ }^{76}$. Vier kleine, flächig mit Kalk behaftete Lampenfragmente können hingegen innerhalb der Spätantike nicht genauer eingeordnet werden. Hervorzuheben ist ein kleiner Glasarmring (Dm 4,2 cm; Kat. 42) 7 $^{77}$. Auf umgelagerten Werkstattschutt lässt auch hier wieder ein Randfragment eines Gusstiegels schließen. Insgesamt ist trotz der aufgrund der wenigen feinchronologisch aussagekräftigen Funde gebotenen Vorsicht von einer Datierung der SE 20 in das 4. Jahrhundert auszugehen.

SE 26 enthielt nur kleinteilig gebrochene Amphorenwand- und Henkelfragmente ${ }^{78}$, ein nicht näher bestimmbares kleines Lampenfragment und zwei kleine bis mittelgroße Wandfragmente von Küchenwaregefäßen. Ein Röhrchenstandring eines Kelchglases ${ }^{79}$ verweist in die Spätantike (Kat. 43). Es kann also nur eine allgemeine Datierung in die Spätantike vorgeschlagen werden, genauere Aussagen lässt das spärliche Fundmaterial nicht zu. Aufschlussreicher sind hingegen die Münzen aus SE 26: Eine davon ist Constantius II (341-348 n. Chr.) zuzuweisen, die zweite ist ein Centenionalis-Fragment aus der zweiten Hälfte des 4. Jahrhunderts $^{80}$. Man wird also nicht fehlgehen, SE 26 in die zweite Hälfte des 4. Jahrhunderts zu datieren.

Aus dem Nutzungshorizont SE 28 stammen ebenfalls keine feinchronologisch aussagekräftigen Feinwaren. Der Rand eines Kochtopfes mit deutlich abgesetztem Rand und geripptem Wandansatz (Kat. 44) kann allerdings anhand von Vergleichen ${ }^{81}$ an das Ende des 4. bzw. in die erste Hälfte des 5. Jahrhunderts datiert werden. Ein Amphorenrandfragment mit leicht eingebogenem Rand und beidseitig verdickter Lippe (Kat. 45) ist hingegen typologisch nicht näher bestimmbar. SE 28 beinhaltete außerdem sechs kleine Lampenfragmente, die innerhalb der Spätantike nicht näher eingeordnet werden können (Kat. 46. 47). Das Henkelfragment Kat. 47 dürfte allerdings zu einer mehrschnauzigen Lampe gehören ${ }^{82}$. Der Röhrchenstandring eines Glasbechers (Kat. 48) stellt hingegen eine weitläufige Form dar und ist nicht näher zuzuweisen. Insgesamt wird auch dieser zur Kalklöschgrube gehörige Nutzungshorizont in das ausgehende 4. bzw. beginnende 5. Jahrhundert zu datieren sein.

Aus der SE 31 - einem weiteren Nutzungshorizont der Kalklöschgrube - stammen ca. 200 registrierte Funde hauptsächlich keramischer Natur, die meist kleinteilig gebrochen und teilweise versintert sind. Es wurde keine chronologisch aussagekräftige Feinware geborgen, wohl aber zwei Randfragmente von Kochtöpfen, die dem Ende des 4./Anfang des 5. Jahrhunderts zuzurechnen sind (Kat. 49. 50) 8. $^{83}$ Erwähnenswert ist außerdem ein gelochter Lampengriff mit (noch schwach sichtbarem) Tannenreisdekor und Resten eines matthellen Überzugs (Kat. 51). Außerdem wurden drei zusammengehörige Wandfragmente einer LR 1-Amphore geborgen, deren rötliches Fabric mit feinen weißen Einschlüssen möglicherweise auf einen samischen Produktionsort schließen lässt ${ }^{84}$; dennoch kann das Fragment nicht näher als zwischen dem 4. und 7. Jahrhundert eingeordnet werden. Viele der klein gebrochenen Amphorenwandfragmente entsprechen in ihrem Fabric dem Amphorentyp LRA 3, können aber innerhalb der Spätantike nicht näher zeitlich eingegrenzt werden. Die Glasfunde - u. a. ein kleiner Becher mit vollem Standring (Kat. 52) und ein weiterer Becher oder eine Schale mit einem blauen, massiven Standring (Nr. 53) ${ }^{85}$ - sind in das ausgehende 3. und in das 4. Jahrhundert zu datieren.

\footnotetext{
${ }^{74}$ Ladstätter 2008, 180-182; Bezeczky 2005, 204 f.; Ladstätter 2007, 212.

${ }^{75}$ Freundliche Information S. Ladstätter, unpubliziert.

${ }^{76}$ Bass - van Doorninck 1971, 34 Taf. 2, Abb. 8-12.

77 Vgl. z. B. Miltner 1937, Nr. 198; Mitsopoulos-Leon 2007, Taf. 33, L 250.

${ }_{78}$ Der Großteil der Amphoren-Wandfragmente kann dem Typ LRA 3 zugewiesen, jedoch nicht näher datiert werden.

${ }^{79}$ Vgl. z. B. Czurda-Ruth 2007, Taf. 32, 735. 738; 33, 644-646. 686. 707-710. 725. 727-729. 753. 754.

${ }^{80}$ Mü 2005/25, Mü 2005/39, s. die Münzliste in Kapitel 4 (M. Pfisterer).

${ }^{81}$ z. B. Turnovsky 2005a, Abb. 1, 2; Ladstätter 2008, Abb. 31, 1-2.

${ }^{82}$ Miltner 1937, Nr. 198; Mitsopoulos-Leon 2007, Taf. 33, L 250; Gassner 1997, Taf. 64, 821; 65, 835; Ladstätter 2005 , K 843.

83 z. B. Turnovsky 2005a, Abb. 1, 2; Ladstätter 2008, Abb. 31, 1-2.

${ }^{84}$ T. Bezeczky sei für diese Information gedankt. Zu Amphoren vom Typ LRA 1 s. Bezeczky 2005, 205 f.; Ladstätter 2008, $182 \mathrm{f}$.

${ }^{85}$ Vgl. z. B. Czurda-Ruth 2007, Taf. 18, 573.
} 
Insgesamt ist für den Nutzungshorizont SE 31 also ein relativ weiter Datierungsrahmen vom ausgehenden 3. bis an den Anfang des 5. Jahrhunderts gegeben. Dennoch passt auch SE 31 zu der Datierung der restlichen zur Kalklöschgrube gehörigen Horizonte oder ist zumindest nicht später zu datieren.

Die Verfüllung der sekundär in die Kalklöschgrube eingetieften Abfallgrube (?) (SE 21) enthielt ein teilweise verbranntes LRC-Randfragment der Form Hayes 4 mit Wandknick im oberen Gefäßdrittel, das in das 5. Jahrhundert datiert werden kann (Kat. 54) ${ }^{86}$. Es fanden sich darin außerdem Fragmente von sechs Lampen, die innerhalb der Spätantike allerdings nicht näher datiert werden können. Die einzige Ausnahme bildet das Fragment Kat. 55, das wohl zu einer Lampe mit Mittelgriff und Doppelschnauze gehört und in das 5. Jahrhundert datieren dürfte ${ }^{87}$.

Zusammenfassend kann festgehalten werden, dass die Kalklöschgrube und die zugehörigen Nutzungshorizonte dem Fundmaterial nach zu schließen in der zweiten Hälfte des 4. bzw. im beginnenden 5. Jahrhundert angelegt worden waren. Im fortgeschrittenen 5. Jahrhundert wurde darin eine Abfallgrube eingetieft, was impliziert, dass die Kalklöschgrube zu diesem Zeitpunkt nicht mehr in Verwendung stand. Der kleinteilige Erhaltungszustand des keramischen Fundmaterials weist auf eine mehrmalige Umlagerung hin. Die endgültige Aufgabe der Grube und der damit verbundenen Tätigkeit des Kalklöschens wird durch die eingebrachte Planierschicht SE 12 im 5. Jahrhundert besiegelt.

\subsection{Fundkomplexe aus der spätantiken Verbauung (SE 12, 8, 34, 11, 10, 7/9, 30, 4, 1, 41, 2/27)}

Die zeitliche Einordnung der Entstehung der Planierung SE 12 gestaltet sich aufgrund des wenigen feinchronologisch auswertbaren Fundmaterials als schwierig. Ein LRC-Randfragment der Form Hayes 3 (Kat. 56) ${ }^{88}$ weist in das 5. Jahrhundert, ein ARS-Bodenfragment, das der Form Hayes 50B zugeordnet werden kann (Kat. 57), datiert in die Zeit von 350-400 n. Chr. ${ }^{89}$. Der erhaltene Oberteil einer Amphore, deren Form zwischen dem >Samos Cistern Type $<$ und dem Typ >Agora M 273 $<$ liegt ${ }^{90}$ (Kat. 58), weist ebenfalls in das 5. Jahrhundert. Ein weiteres Amphorenfragment (Kat. 62) ist dem Typ Agora M273 zuzuordnen, der im 4./5. Jahrhundert auftritt ${ }^{91}$. Das glimmerhaltige Fabric lässt auf eine Herstellung in der lokal-regionalen Umgebung von Ephesos schließen ${ }^{92}$. Ein massiver, nahezu vollständig geschlossener Knauf einer Amphore vom Typ LRA 3 ist ebenfalls dem 5. Jahrhundert zuzuordnen (Kat. 59), für einen weiteren, nicht ganz geschlossenen Fuß ist eine frühe Datierung möglich (Kat. 60) ${ }^{93}$. Mehrere Randfragmente (wie Kat. 61) gehören gleichfalls zu Amphoren vom Typ LRA 3. Unklar ist die Zuordnung des Amphorenrandes Kat. 63, der das typische lokal-regionale spätantike Fabric aufweist, sich aber mit seiner hängenden Lippe und seinem Durchmesser keinem der bekannten lokalen Amphorentypen der Spätantike (LRA 3, Ephesos 56) zuweisen lässt. Zu ergänzen sind außerdem mehrere Fragmente von Lampen, darunter zwei Doppelschnauzenlampen mit jeweils zentralem, gelochtem Henkel und einem davor gesetzten Füllloch, und eine Doppelschnauzenlampe mit Warzendekor auf der Schulter, die in das 5. Jahrhundert datiert werden können (Kat. 64) ${ }^{94}$. Auch die vorhandenen Küchenwaren wie beispielsweise Töpfe/Kasserollen mit gerippter Wand und nach innen abgestuftem Rand (Kat. 65) ${ }^{95}$ und eine Pfanne mit knopfartig verdicktem Rand (Kat. 66) ${ }^{96}$ weisen in das 5. Jahrhundert. Sämtliche bestimmbare Münzfunde aus SE 12 sind Constantius II zuzuordnen und 351-361

\footnotetext{
${ }^{86}$ Vgl. Ladstätter - Sauer 2005, 79.

87 Vgl. Miltner 1937, Nr. 313; Mitsopoulos-Leon 2007, Taf. 33, L 250; Gassner 1997, Taf. 65, 385.

88 Vgl. Ladstätter - Sauer 2005, Taf. 7, 32; Hayes 1972, 329.

89 Hayes 1972, 68-73.

90 Das Fabric macht durch seine glimmrige Beschaffenheit und seine violettbraune Farbe einen lokalen Eindruck. Ich danke T. Bezeczky für den Hinweis. Zu den Amphorentypen s. Bezeczky 2005, 207 f. mit weiterführender Lit.

91 Zum Amphorentyp Agora M 273 s. Reynolds 1998, 157-184. Bonifay - Piéri 1995, 94-120.

92 Ich danke T. Bezeczky für die Diskussion des Stücks und für die Begutachtung der Probe im Polarisationsmikroskop.

93 Ladstätter 2008, 180-182; Ladstätter - Steskal 2009; Ladstätter 2007, 212; Bezeczky 2005, 204.

94 Miltner 1937, Nr. 313; Mitsopoulos-Leon 2007, 87 L 250; Gassner 1997, Taf. 65, 835.

95 Vgl. Turnovsky 2005b, Abb. 4, 1; Ladstätter 2008, K 177. 212. 526.

96 Vgl. Turnovsky 2005b, Abb. 6, 6; Ladstätter 2008, K 181.
} 
n. Chr. geprägt worden ${ }^{97}$. Auffallend ist das Auftreten von acht Gusstiegelfragmenten, die - wie jene Fragmente aus der SE darunter - möglicherweise darauf schließen lassen, dass sich in der Phase vor dem Einbringen der Schuttschicht nördlich des Nymphäums ein Werkstattareal befand, das neben der Kalklöschgrube noch weitere verarbeitende Einrichtungen umfasste. Es ist aber auch hier nicht auszuschließen, dass es sich um umgelagerten Werkstattschutt handelt.

Aus der Auswertung des Fundmaterials lässt sich eine Datierung von SE 12 in die erste Hälfte des 5. Jahrhunderts ermitteln. Durch die >Versiegelung « mit der Brandschuttschicht SE 12 ist ein terminus ante quem für die Aufgabe der Kalklöschgrube und die zugehörigen Nutzungshorizonte gegeben.

In der Verfüllung des Wasserbeckens (SE 8) wurden chronologisch empfindliche Feinwaren geborgen, die eine zeitliche Einordnung der Aufgabe des Wasserbeckens erlauben. SE 8 enthält zwei Randfragmente von LRC-Tellern der Form Hayes 3/10 (Kat. 67. 68) ${ }^{98}$, die in das 6. Jahrhundert zu datieren sind. Ein Rand- und ein Bodenfragment von ERSW-Tellern der Form Hayes 3 (Kat. 69. 70) sowie ein weiteres ERSW-Bodenfragment der Form Hayes 3 mit dem Rest eines Kreuzstempels (Kat. 71) weisen ebenfalls in das 6. Jahrhundert $^{99}$. Bei einem ARS-Bodenfragment, das höchstwahrscheinlich der Form Hayes 58 zugeschrieben werden kann, handelt es sich hingegen um ein >Altstück sind außerdem Fragmente von zwei großen Tellern unterschiedlicher Form, die einer bislang typochronologisch nicht näher definierten $>$ Mäandertalsigillata zugeordnet werden können ${ }^{101}$. Die Gefäße bestehen aus einem mit feinem Glimmer und feinen weißen Einschlüssen durchsetzten, porösen Ton und sind flächig mit einem dunkelroten, matt glänzenden Überzug versehen. Kat. 72 ist ein großer Teller mit eingebogenem Rand und außen verdickter, abgerundeter und facettenartig gestufter Lippe ${ }^{102}$. Auf der Bodeninnenseite weist das Gefäß einen feinen Ratterdekor auf ${ }^{103}$. Kat. 73 ist ebenfalls ein Teller, allerdings mit hängendem breitem, außen profiliertem Rand; für dieses Gefäß konnten bislang keine Parallelen gefunden werden. An Amphorenfragmenten ist ein Randfragment vom Typ LRA 1 zu nennen (Kat. 74), der vom ausgehenden 4. bis in die Mitte des 7. Jahrhunderts produziert wird ${ }^{104}$, außerdem ein Rand einer Amphore vom Typ LRA 3 (Kat. 75) sowie ein weiteres Randfragment vom Typ Ephesos 56 (Kat. 76) und zwei nicht zur Gänze geschlossene Knauffragmente vom Typ LRA $3^{105}$ (wie Kat. 77). Zu erwähnen ist zudem ein Wandfragment einer LR 3Amphore, das wiederum ein Graffito aufweist (Kat. 78). Es handelt sich - laut H. Taeuber - um einen Brief oder eine Botschaft; in der zweiten Zeile dürfte »ich [oder: sie] habe[n] ver[- bzw. hinter]lassen« gelesen werden ${ }^{106}$.

Aus der Zusammensetzung des Fundmaterials und speziell aufgrund der jüngsten in SE 8 auftretenden Funde ist insgesamt von einer Verfüllung des Wasserbeckens im 6. Jahrhundert auszugehen.

In das ausgehende 5. oder die erste Hälfte des 6. Jahrhunderts dürfte die Verfüllung der ausgerissenen Rohrgrube (SE 34) im Anschluss östlich an das Wasserbecken zu datieren sein. Dafür spricht vor allem das Randfragment eines ARS-Tellers, das entweder eine Variante der Form Hayes $91^{107}$ darstellt oder der Form Hayes $96^{108}$ zuzuordnen ist (Kat. 79). Zwei LRC-Bodenfragmente der Form Hayes 4 datieren in das 5. Jahrhundert (Kat. 80. 81) ${ }^{109}$. Ein ERSW-Bodenfragment (Kat. 82) ${ }^{110}$ und ein Wandfragment einer frühby-

\footnotetext{
${ }^{97}$ Mü 2005/13-15, Mü 2005/35-36, s. die Münzliste in Kapitel 4 (M. Pfisterer).

98 Vgl. Ladstätter - Sauer 2005, Taf. 6, 76. 77.

$99 \mathrm{Zu}$ Definition, Formen- und Dekorspektrum der ERSW s. Ladstätter - Sauer 2005, 152-154.

100 Wegen seines kleinteiligen Erhaltungszustandes wurde es nicht in den Katalog aufgenommen.

101 S. Ladstätter - B. Pichler - R. Sauer, Rohstoffsurvey 2001 und 2003 im Umland von Ephesos (unpubliziert). Zur >Mäandertalsigillata< s. Hayes 1972, 408-410; Ladstätter 2008, 115.

102 Die Form erinnert an spätantike Feinwareteller aus Hierapolis, die aus einem »Asia Minor fabric« bestehen: Cottica 2000, Abb. 3, 21-22.

103 Vgl. eventuell Turnovsky 2005b, Abb. 8, 1: »red slip ware with pricked decoration $\left(1^{\text {st }}\right.$ half $7^{\text {th }} \mathrm{c}$. AD) «.

104 Bezeczky 2005, 205 f.; Ladstätter 2008, 182 f.

105 Bezeczky 2005, 204; Ladstätter 2008, 180-182.

${ }^{106}$ Ich bedanke mich wiederum herzlich bei H. Taeuber für die Bestimmung des Graffitos.

107 Vgl. Hayes 1972, 144

108 Hayes 1972, 149 f. Abb. 27.

109 Hayes 1972, 338; Ladstätter - Sauer 2005, Taf. 6, 78-82.

${ }^{110}$ Lokale Imitationen von LRC-Gefäßen sind in Ephesos vor allem ab dem 6. Jh. ein Bestandteil des Feinwaregeschirrs. Ladstätter Sauer 2005, 153 .
} 
zantinischen Ampulle ${ }^{111}$ weisen hingegen wiederum in das 6. Jahrhundert. Auch ein Henkelfragment einer LR 2-Amphore (Kat. 83) ${ }^{112}$ widerspricht einer Datierung von SE 34 in das 6./7. Jahrhundert nicht. Von den kleinteilig gebrochenen Lampenfragmenten ist nur eine rot überfangene, kleine Lampe mit massivem Griffzapfen zu bestimmen (Kat. 84) ${ }^{113}$. Sie ist flächig versintert und kann innerhalb der Spätantike nicht näher datiert werden. Unter den Glasfunden befinden sich der Rand eines Bechers mit leicht verdicktem, eingebogenem Rand (Kat. 85) ${ }^{114}$ und ein Röhrchenstandring eines Kelchglases (Kat. 86) ${ }^{115}$. In SE 34 fanden sich im Übrigen wieder Hinweise auf Produktionsstätten in Form (umgelagerten?) Werkstattschutts, so z. B. Gusstiegelfragmente.

Insgesamt kann davon ausgegangen werden, dass die Grube der östlich des Wasserbeckens ausgerissenen Leitung im ausgehenden 5. Jahrhundert bzw. in der ersten Hälfte des 6. Jahrhunderts mit SE 34 verfüllt wurde.

Der Verfüllungszeitraum der Abfallgrube SE 11 kann durch die Auswertung des keramischen Fundmaterials nicht mit Sicherheit bestimmt werden, da feinchronologisch aussagekräftige Gattungen fehlen. Es kann jedoch auf ein Fragment einer sog. One Handle Jar verwiesen werden, welche die Vorläufer der LR 3-Amphoren darstellen und bis in das 4. Jahrhundert auftreten (Kat. 87) ${ }^{116}$. Ein Randfragment eines Topfes mit innen abgestuftem Rand kann nach Parallelen in das ausgehende 4. und die erste Hälfte des 5. Jahrhunderts datiert werden (Kat. 88) ${ }^{117}$. Erwähnenswert sind auch sieben Lampenfragmente, die in die Spätantike datiert werden können. Eines davon ist rot überzogen und zeigt den Rest eines planta pedis-Stempels auf der Unterseite (Kat. 89) ${ }^{118}$, ein weiteres, rot überfangenes Lampenfragment weist eine Rosette als Diskusdekor auf (Kat. 90) ${ }^{119}$

Zwei Münzen des Constantius II (351-355 n. Chr.) und des Valentinianus II (388-392 n. Chr.) ${ }^{120}$ weisen eindeutig in die zweite Hälfte bzw. an das Ende des 4. Jahrhunderts. Die Abfallgrube muss, der Fundzusammensetzung nach zu schließen, mit umgelagertem Schutt aus dem Ende des 4. Jahrhunderts verfüllt worden sein. Aus SE 12 ergibt sich die erste Hälfte des 5. Jahrhunderts als terminus post quem.

Maßgeblich für die zeitliche Einordnung der Aufschüttung/Abfallgrube (?), in welcher der Kopf einer Kopie des Doryphoros aus antoninischer Zeit ${ }^{121}$ gefunden wurde (SE 10), sind vor allem zwei Randfragmente Afrikanischer Sigillata-Teller der Formen Hayes $108^{122}$ bzw. Hayes $105^{123}$ (Kat. 91. 92) aus dem Westteil von Schnitt 1. Beide weisen an das Ende des 6. bzw. in die erste Hälfte des 7. Jahrhunderts. Älter ist hingegen Kat. 93, ebenfalls ein ARS-Teller, allerdings der Form Hayes 50A (ca. 300-360 n. Chr.) ${ }^{124}$. In SE 10 fand sich außerdem ein Tellerrand der LRC, welcher der Form Hayes 3/10 zuzurechnen ist. Der Rand ist blockartig verdickt, außen ist eine leichte Kerbreihe sichtbar (Kat. 94). Das Fragment dürfte aufgrund von Parallelen ${ }^{125}$ in das 6. Jahrhundert zu datieren sein. Kat. 95 ist hingegen der Form Hayes 3 zuzuordnen und datiert in das 5./6. Jahrhundert ${ }^{126}$. Ein Wandfragment einer frühbyzantinischen Ampulle sowie ein wohl zu einer Amphore vom Typ Ephesos 56 gehöriges, dickwandiges Knauffragment (Kat. 96) ${ }^{127}$ weisen in das

${ }_{111}$ Das vorliegende Stück ist aufgrund seiner glimmerlosen Beschaffenheit eindeutig als Import anzusprechen. Vgl. Metaxas 2005, 101-103; Ladstätter - Steskal 2009.

112 Bezeczky 2005, $206 \mathrm{f}$.

113 Vgl. Miltner 1937, 243; Ladstätter 2008, 117 f. (Typ I).

114 Vgl. Isings 1957, 106 a/b; Steskal 2008, G 148.

115 Vgl. z. B. Czurda-Ruth 2007, Taf. 32. 33.

116 Vgl. Bezeczky 2005, $203 \mathrm{f}$.

117 Vgl. Turnovsky 2005a, Abb. 1, 1. Vgl. auch Gassner 1997, Nr. 723. 724. 726; Ladstätter 2008, Abb. 31, 3.

118 Miltner 1937, Typ III Form 3; Mitsopoulos-Leon 2007, L 239.

${ }_{119}$ Vgl. Ladstätter - Sauer 2008, K 169.

${ }^{120}$ Mü 2005/10, Mü 2005/34, s. die Münzliste in Kapitel 4 (M. Pfisterer).

${ }^{121}$ s. Beitrag M. Aurenhammer.

${ }^{122}$ Hayes 1972, 171.

${ }^{123}$ Hayes 1972, 169.

124 Hayes 1972, 73.

${ }^{125}$ Vgl. Ladstätter 2005, Taf. 6, 76 (allerdings ohne Kerbdekor).

${ }^{126}$ Hayes 1972, 329-338; Ladstätter - Sauer 2005, 159 f.

${ }^{127}$ Vgl. etwa Bezeczky 2005, Taf. 2, Abb. 17. Aufgrund der Dickwandigkeit des Stücks ist allerdings auch nicht auszuschließen, dass es sich um einen sog. Ephesischen Amphoriskos aus dem 6./7. Jh. handelt, selbst wenn der für diese Gattung typische grobe, gedrehte Fuß fehlt. Zu Definition, Typologie und Datierung >Ephesischer Amphoriskoi々s. Metaxas 2005, 97-100. 
6. Jahrhundert. Die restlichen Amphorenfragmente sind meist nicht zuordenbar oder innerhalb des späten 4. und beginnenden 5. Jahrhunderts bis Anfang des 7. Jahrhunderts nicht näher einzuordnen. Vertreten sind aber beispielsweise zwei (späte) Ränder vom Typ LRA 3 (Kat. 97. 98) ${ }^{128}$. Es konnten außerdem insgesamt 47 Fragmente spätantiker Lampen in dieser Schicht geborgen werden, darunter mehrere gelochte Griffe und Schulterfragmente von Mehrfachschnauzenlampen ${ }^{129}$ (Kat. 99), ein Schnauzenfragment mit Resten eines Fischgrätmusters (Kat. 100) ${ }^{130}$, Fragmente von Lampen mit Warzendekor sowie ein Lampenboden mit Bodenrosette (Kat. 101) ${ }^{131}$. Unter den Glasfunden ist ein abgerundeter Boden zu nennen, der wohl zu einer Lampe mit Stielfuß gehört, wie sie ab dem 4./5. Jahrhundert verbreitet sind (Kat. 102) ${ }^{132}$. Auch in SE 10 sind wieder Produktionszeugnisse in Form von Rand-, Boden- und Wandfragmenten von Gusstiegeln vorhanden. Das Gebrauchskeramik- und Küchenwarespektrum entspricht - soweit zuordenbar - dem spätantiken Inventar.

Insgesamt enthielt diese Schicht ca. 1100 Objekte, wobei es sich vielfach um kleinteilig gebrochene, abgenutzte, nicht anpassende Fragmente handelt. Es ist also auch hier eine mindestens einmalige Umlagerung vorauszusetzen, ehe die Funde in die Aufschüttung SE 10 gelangten. Diese kann daher erst am Ende des 6. oder Anfang des 7. Jahrhunderts entstanden sein, worauf auch die jüngsten Funde schließen lassen. Möglicherweise handelt es sich einmal mehr um umgelagerten Werkstattschutt, wofür das Auftreten der Gusstiegelfragmente sprechen würde.

Die Aufschüttung SE 7/SE 9 ist - vor allem aufgrund des Fundes eines LRC-Tellers der Form Hayes $10 \mathrm{~A}^{133}$ - dem späten 6. Jahrhundert zuzuordnen (Kat. 103). Das Feinkeramikspektrum umfasst außerdem mehrere LRC-Tellerränder der Form Hayes 3 (Kat. 104. 105), die in das fortgeschrittene 5. und frühe 6. Jahrhundert datiert werden können ${ }^{134}$, mehrere Böden solcher Teller (Kat. 106. 107) sowie ein kleines, stark versintertes Schälchen der Form Hayes 2, das bereits in das späte 4. oder in die erste Hälfte des 5. Jahrhunderts datiert (Kat. 108) $)^{135}$. In das ausgehende 5./6. Jahrhundert verweist außerdem ein Fragment einer frühbyzantinischen Ampulle (Kat. 109) ${ }^{136}$. Dieser Gefäßtyp wurde ab dem späten 5. Jahrhundert nach Ephesos importiert ${ }^{137}$ und schließlich auch lokal imitiert ${ }^{138}$. Ein Amphorenrand vom Typ Ephesos 56 (Kat. 110) kann in das 6. Jahrhundert datiert werden ${ }^{139}$, außerdem treten mehrere Randfragmente von Amphoren des Typs LRA 3 auf (wie Kat. 111). Hinzuweisen ist auch auf einen eiförmigen Glasbecher mit ausbiegendem, außen durch Schliffrillen profiliertem Rand (Kat. 112) ${ }^{140}$. Im Übrigen wurden auch in dieser Schicht wieder Fragmente von Gusstiegeln gefunden.

Die einzige Münze aus SE 7 datiert zwar in die Zeit von 348-355 n. Chr. ${ }^{141}$, insgesamt ist die Aufschüttung SE 7/SE 9 aber aufgrund des keramischen Fundmaterials in das späte 6. Jahrhundert zu setzen.

Ausschlaggebend für die Datierung von SE 30 ist besonders der Rand eines Afrikanischen Sigillata (ARS)-Tellers, der zwar aufgrund seiner Kleinteiligkeit nicht in den Katalog aufgenommen wurde, aber der Form Hayes 105 zugeschrieben werden kann. Das stark verbrannte Stück datiert in die Zeit um 580/600-660 n. Chr. ${ }^{142}$. Der ARS-Teller Kat. 113 entspricht hingegen der Form Hayes 57 und wurde höchstwahrschein-

\footnotetext{
128 Vertreten sind auch Wandfragmente von Amphoren der Typen LRA 1-4, vgl. Bezeczky 2005, 204 f. 208.

129 Vgl. z. B. Miltner 1937, Nr. 313.

${ }^{130} \mathrm{Zu}$ diesem Fragment konnte bislang keine exakte Parallele gefunden werden.

131 Vgl. Miltner 1937, Taf. 10, 412; Mitsopoulos-Leon 2007, L 250.

132 Vgl. z. B. Czurda-Ruth 2007, 181-184 bes. Taf. 21, 823.

133 Vgl. Ladstätter - Sauer 2005, Taf. 7, 89; Hayes 1972, 343-346.

134 Vgl. Hayes 1972, 329-338; Ladstätter - Sauer 2005, 159 f.

135 Hayes 1972, 327-329.

136 J. W. Hayes führte die Fundgattung als »Late Roman unguentaria« in die Forschung ein, da die Form der Gefäße an hellenistische Unguentaria erinnert. Hayes 1968, 203-216; Hayes 1971, 243-247. Die aktuellsten wissenschaftlichen Arbeiten über frühbyzantinische Ampullen und Amphoriskoi stellen Metaxas 2005 und Sauer - Ladstätter 2005 dar.

137 Aufgrund mineralogisch-petrographischer Analysen kommen Nordsyrien, Kilikien, Rhodos oder Zypern als Produktionsgegenden in Frage: S. Ladstätter, Archäologische Interpretation, in: Sauer - Ladstätter 2005, 133.

${ }^{138} \mathrm{Zu}$ den >ephesischen< frühbyzantinischen Ampullen s. Metaxas 2005, 95-97.

139 Bezeczky 2005, 204 f.; Ladstätter - Steskal 2009.

140 Vgl. Harter 1999, 70-72 (Form 815); Czurda-Ruth 2007, Taf. 14, 376-381.

141 Mü 2005/9, s. die Münzliste in Kapitel 4 (M. Pfisterer).

${ }^{142}$ Hayes 1972, 166-169.
} 
lich bereits in der Zeit von 325-400 n. Chr. produziert ${ }^{143}$. Fragmente von LRC-Gefäßen, die hauptsächlich unterschiedlichen Varianten der Form Hayes 3 zugeschrieben werden können (vertreten ist aber auch eine Variante der Tellerform Hayes 8), weisen zumindest in die zweite Hälfte des 5. Jahrhunderts ${ }^{144}$. Eine ERSW-Steilrandschale (Kat. 114), die möglicherweise die ARS-Form Hayes 91/92 imitiert ${ }^{145}$, und eine Imitation eines Tellers der Form Hayes 1B (Kat. 115) ${ }^{146}$ deuten wiederum in das 5.-6. Jahrhundert. Auch zwei geschlossene Knäufe von Amphoren des Typs LRA 3 können dem 6. Jahrhundert zugewiesen werden ${ }^{147}$. Hervorzuheben sind außerdem ein Randfragment (Kat. 116) und ein aus mehreren Stücken bestehendes Wandfragment frühbyzantinischer Ampullen ${ }^{148}$. Die Stücke aus SE 30 können aufgrund ihres glimmerlosen Tons als Importe gelten. Es fanden sich in SE 30 außerdem mehrere kleine Fragmente spätantiker Lampen, aber auch eine ganz erhaltene, rot überzogene Lampe mit Griffzapfen (Kat. 117) ${ }^{149}$. Das Glasspektrum, das u. a. zwei Standfüße von Kelchgläsern sowie mehrere Becherränder umfasst, fügt sich in den zeitlichen Rahmen ein.

Man wird insgesamt nicht fehlgehen, SE 30 in das ausgehende 6. und beginnende 7. Jahrhundert zu setzen.

Für eine absolutchronologische Einordnung der massiven Aufschüttung SE 4 zeichnen in erster Linie die darin vorkommenden feinkeramischen Waren verantwortlich. Dazu gehören drei Randstücke von LRC-Tellern der Form Hayes 3, wobei Kat. 118 und 119 der Variante Hayes $3 \mathrm{~F}$ zugeordnet und in das 6. Jahrhundert datiert werden können ${ }^{150}$. Das jüngste Stück, Kat. 120, entspricht der Variante Hayes 3 H, die ebenfalls eine späte Ausbildung darstellt und im 6. Jahrhundert auftritt ${ }^{151}$. Drei Bodenfragmente (wie Kat. 121) sind allgemein der Form Hayes 3 zuzuordnen ${ }^{152}$. Bereits in das fortgeschrittene 6. Jahrhundert dürften zwei lokale Imitationen (ERSW) der LRC-Tellerformen Hayes 8 (Kat. 122) ) $^{153}$ und Hayes 10 (Kat. 123) ) $^{154}$ weisen. Auch ein Randfragment (Kat. 124) sowie der abgebrochene Knauf einer Amphore vom Typ Ephesos 56 (Kat. 125) datieren in das 6./7. Jahrhundert ${ }^{155}$. Das hier nicht näher behandelte Küchenware- und Gebrauchskeramikspektrum entspricht dieser Zeitstellung. In ein Wandfragment einer Amphore vom Typ LRA 3 ist offensichtlich ein Brief eingeritzt (Kat. 126). Als Inhalt schlägt H. Taeuber »Er möge sich um die üblen (Kerle?) kümmern« vor ${ }^{156}$.

Es kann zweifelsohne davon ausgegangen werden, dass die Aufschüttung SE 4 im ausgehenden 6./beginnenden 7. Jahrhundert erfolgte. Durch SE 10, jener Aufschüttung, in welcher der Kopf des Doryphoros gefunden wurde, und SE 30, die jeweils unter SE 4 liegen, ist ein terminus post quem gegeben. Die Aufschüttungen dürften also unmittelbar aufeinander erfolgt sein.

Die mörtelige Unterkonstruktion für den letzten Boden, SE 1, bildet eine der quantitativ umfangreichsten fundführenden Schichten der spätantiken Nutzung des Areals nördlich des Nymphaeum Traiani ${ }^{157}$. Sie enthält u. a. LRC-Tellerrandfragmente der Formen Hayes 3F (Kat. 127. 128) ) $^{158}$ und Hayes 3/10 (Kat. 129) ein Randfragment eines LRC-Tellers der Form Hayes 6 (Kat. 130) ${ }^{160}$ und zwei Bodenfragmente von LRC-

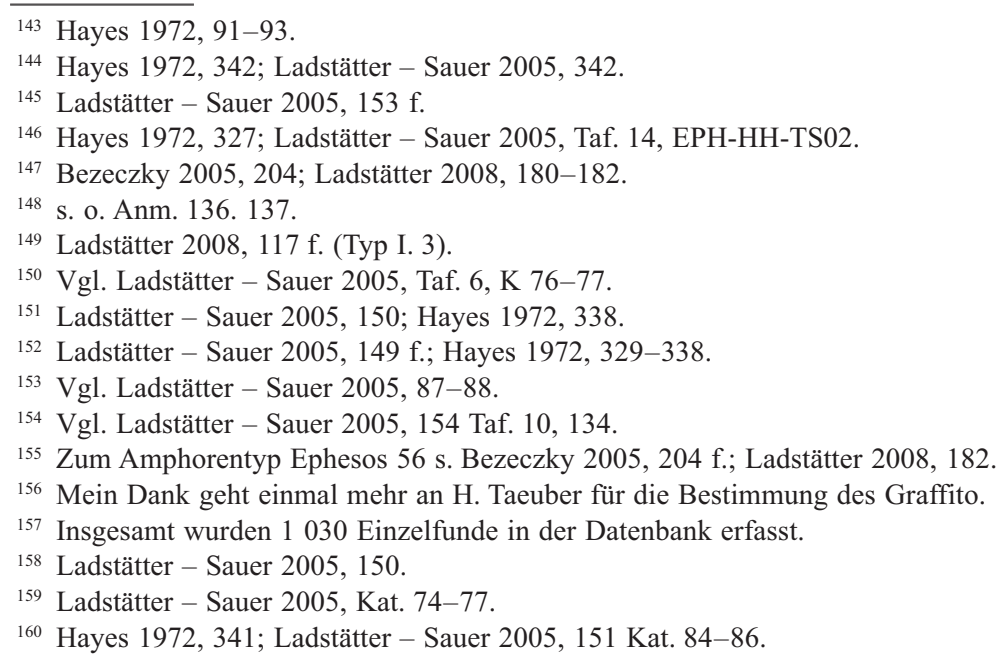


Tellern, die jeweils Stempelreste aufweisen (Kat. 131. 132). Vertreten sind auch Imitationen von LRC-Tellern der Form Hayes 3 in lokaler ERSW (Kat. 133. 135) ${ }^{161}$. Ein Randfragment, das einen Kerbdekor an der Randaußenseite aufweist, stellt eine Imitation/Variante der LRC-Form Hayes 8 dar (Kat. 134) ${ }^{162}$. Die Originalform tritt bereits in der zweiten Hälfte des 5. Jahrhunderts auf, die Imitation ist wohl in das 6. Jahrhundert zu datieren. Die LRC- und ERSW-Funde sind insgesamt dem 6. bzw. fortgeschrittenen 6. Jahrhundert $\mathrm{n}$. Chr. zuzuordnen. Maßgeblich für die absolutchronologische Einordnung der SE 1 sind jedoch ein Fragment einer ARS-Schüssel der Form Hayes 108, die vom Ende des 6. bis in die Mitte des 7. Jahrhunderts hergestellt wird (Kat. 136) ${ }^{163}$, und ein ARS-Bodenfragment der Form Hayes 104C (Kat. 137), welches der Zeit von der Mitte des 6. bis zum Anfang des 7. Jahrhunderts zugerechnet werden kann ${ }^{164}$. In diesem Zusammenhang ist ein weiteres Randfragment eines rötlich überzogenen Tellers lokalen Fabrics anzuführen, das möglicherweise eine ältere ARS-Form (Hayes 9B ${ }^{165}$ ) imitiert (Kat. 138). In das 6./7. Jahrhundert ist das Randfragment einer frühbyzantinischen Ampulle zu datieren, die aufgrund ihres geringen Glimmergehaltes als Import angesprochen werden kann (Kat. 139) ${ }^{166}$. Ein geschlossener Knauf gehört zu einer lokal produzierten Amphore vom Typ LRA 3 (Kat. 140) ${ }^{167}$. Hinzuweisen ist außerdem auf ein weiteres LRA 3-Wandfragment mit einem Graffito (Kat. 141). Dieses beinhaltet möglicherweise eine Mitteilung über einen »-os, der einen Polos [Sonnenuhr] geschickt hat «168.

Zusammenfassend kann festgehalten werden, dass SE 1 in der ersten Hälfte des 7. Jahrhunderts entstanden sein muss. Dafür sprechen vor allem die enthaltenen Fragmente von ARS-, LRC- und ERSW-Gefäßen, aber auch die im Fundspektrum vertretenen Amphoren. Das Küchenware- und Gebrauchskeramikspektrum sowie die zahlreichen kleinen Fragmente spätantiker Lampen aus SE 1 entsprechen ebenfalls dem typischen Fundbild des 6./beginnenden 7. Jahrhunderts ${ }^{169}$ und bestätigen somit die aus der Feinkeramik gewonnene Datierung. Das Spektrum der Glasfunde ${ }^{170}$ spricht ebenfalls nicht gegen eine Zuordnung der Schicht zum ausgehenden 6. und frühen 7. Jahrhundert. Eine in SE 1 gefundene Münze datiert hingegen früher: Sie ist Honorius zuzuweisen und wurde $395-401 \mathrm{n}$. Chr. geprägt ${ }^{171}$.

Der Großteil des Materials ist klein gebrochen, es haben sich keine Ganzgefäße und nur selten anpassende Stücke erhalten, wenige Fragmente ließen sich als zusammengehörig identifizieren. Daher ist davon auszugehen, dass es sich um einen mindestens einmal umgelagerten spätantiken Hausrat handelt, der im Zuge der Fundamentierung für einen Plattenboden (?) in die sandige, mörtelige Erdschicht gelangte. Die zeitliche Stellung des keramischen Materials ist in sich relativ homogen, bis auf wenige Ausnahmen sind keine Funde vor dem ausgehenden 6. Jahrhundert anzusiedeln.

Aus SE 41 (FK 73) unterhalb der Mauer M3 stammen nur wenige Funde, die der römischen Kaiserzeit zugeordnet, feinchronologisch aber nicht näher datiert werden können. Die mit dem keramischen und gläsernen Fundmaterial vergesellschaftete Münze weist hingegen eindeutig in die Spätantike, sie ist der Regierungszeit Arcadius', Theodosius' oder Honorius' zuzuweisen und wurde 404-406 n. Chr. geprägt ${ }^{172}$. Auf jeden Fall ist durch die Datierung von SE 1 in das ausgehende 6./beginnende 7. Jahrhundert ohnehin ein terminus post quem für SE 41 gegeben.

\footnotetext{
${ }^{161}$ Ladstätter - Sauer 2005, $153 \mathrm{f}$.

${ }^{162}$ Hayes 1972, 342; Ladstätter - Sauer 2005, 151 Kat. 88.

${ }^{163}$ Hayes 1972, 172.

164 Hayes 1972, 166.

${ }^{165}$ Hayes 1972, 35-37 (2. Hälfte 2. Jh. n. Chr.).

166 Metaxas 2005, 102 f. s. auch o. Anm. 136. 137.

167 Ladstätter 2008, 180-182; Bezeczky 2005, 204.

${ }_{168}$ Transkription s. Katalog. Für die Bestimmung des Graffitos bin ich H. Taeuber zu Dank verpflichtet.

${ }^{169}$ Funde mehrerer Kochtöpfe mit aufgebogenem Rand und Deckelfalz haben z. B. Parallelen in Fundkomplexen der Marienkirche aus der 1. Hälfte des 7. Jhs. Vgl. Turnovsky 2005a, 636.

170 Vertreten sind beispielsweise drei Standringe von Kelchgläsern (vgl. z. B. Czurda-Ruth 2007, Taf. 18, 626 - Taf. 20, 782) und ein Stabhenkel einer Glaslampe (vgl. z. B. Czurda-Ruth 2007, 177 f. Taf. 21, 798).

${ }^{171}$ Mü 2005/31, s. die Münzliste in Kapitel 4 (M. Pfisterer).

172 Mü 2005/30, s. die Münzliste in Kapitel 4 (M. Pfisterer).
} 
Ausschlaggebend für die Datierung der SE 2 sind die jüngsten Funde aus den entsprechenden Fundkomplexen, wobei besonders ein LRC-Randfragment der Form Hayes $10 \mathrm{C}$ (Kat. 142) sowie eine Imitation eines solchen Tellers (Kat. 143) ${ }^{173}$ zu nennen sind, die jeweils in die erste Hälfte des 7. Jahrhunderts verweisen. Ein LRC-Bodenfragment der Form Hayes 3-6, dessen Innenseite Reste eines zoomorphen Stempels in Form eines Pfaus aufweist ${ }^{174}$ (Kat. 144), ein ERSW-Bodenfragment der Form Hayes 3 (Kat. 145) ${ }^{175}$ und ein geschlossener Knauf einer Amphore vom Typ LRA 3 (Kat. 146) ${ }^{176}$ sind zumindest in das 6. Jahrhundert zu datieren. Ein Randfragment eines kleinen Spatheions (Kat. 147) ${ }^{177}$ stellt den spätesten Fund aus SE 2/27 dar. Es kann dem kleinen Spatheion-Typ 3/Variante C nach M. Bonifay zugeordnet werden, der ab der zweiten Hälfte des 7. Jahrhunderts produziert wird ${ }^{178}$. Eine rot überfangene Lampe mit vollem Griffzapfen und einem feinen fünfreihigen Warzendekor auf der Schulter (Kat. 148) kann in das 6./7. Jahrhundert datiert werden ${ }^{179}$.

Es ist insgesamt also festzuhalten, dass SE 2/27 offensichtlich erst in der zweiten Hälfte des 7. Jahrhunderts entstand und die jüngste nachgewiesene Aktivität im Bereich nördlich des Nymphaeum Traiani darstellt. Obwohl es sich um einen Einzelbefund handelt, ist er dennoch beachtlich, reichen doch die Funde aus dem Bereich der im Osten an das Nymphäum anschließenden Tabernenreihe in der Nordhalle der Kuretenstraße gesichert bislang nur bis in die erste Hälfte des 7. Jahrhunderts ${ }^{180}$.

Zusammenfassend ist zu sagen, dass sich über die Auswertung der Fundkomplexe drei Phasen der Nutzung des Areals nördlich des Nymphaeum Traiani nachweisen lassen, die von der severischen Zeit über die zweite Hälfte des 4. Jahrhunderts und das 5. Jahrhundert bis in das 6. Jahrhundert/die erste Hälfte des 7. Jahrhunderts reichen. Eine letzte Aktivität ist für die zweite Hälfte des 7. Jahrhunderts bezeugt (SE 2).

Das Fundmaterial ist mit wenigen Ausnahmen kleinteilig gebrochen, was impliziert, dass der ursprüngliche Verwendungskontext für die meisten Gefäße außerhalb des jeweiligen Fundzusammenhangs zu suchen sein dürfte.

Was die Zusammensetzung des Fundmaterials in den spätantik-byzantinischen Schichten betrifft, so ergeben sich durchweg Parallelen zu ephesischen Fundkontexten ähnlicher Zeitstellung ${ }^{181}$. Auffallend ist das gehäufte Auftreten von Gusstiegeln, die bisweilen gut erhalten sind und z. T. noch Reste bislang unbestimmter geschmolzener Substanzen enthalten. Dies ist kein vereinzelter Befund, sondern aufgrund weiterer zahlreicher Gusstiegelfunde in den Taberneneingängen unmittelbar östlich des Nymphaeum Traiani muss für die letzten Phasen der Kuretenstraße wohl von einer großflächigen Nutzung der die Straße flankierenden Bereiche als Handwerksareal ausgegangen werden ${ }^{182}$. Der Befund einer Kalkgrube im Bereich nördlich des Nymphäums spricht ebenfalls für eine solche Interpretation.

\footnotetext{
173 Vgl. Ladstätter 2005, 154 Kat. 92.

174 Stempelstil Gruppe III, vgl. Ladstätter - Sauer 2005, 152 Kat. 86. 105; Hayes 1972, Stempel Typ 48.

175 Ladstätter - Sauer 2005, 153 f.

176 Ladstätter 2008, 180-182; Bezeczky 2005, 204

177 Hiermit danke ich T. Bezeczky für Hinweise zu diesem spätesten Spatheion-Typ.

178 Bonifay 2004, 127-129 bes. 129.

179 Vgl. Ladstätter 2008, Kat. 331-333; Meriç 2002, L 109; vgl. Bass - van Doorninck 1982, 12.

180 Iro - Schwaiger - Waldner 2009.

181 Vgl. Ladstätter 2008; Iro - Schwaiger - Waldner 2009.

182 Iro -Schwaiger - Waldner 2009.
} 


\section{Katalog}

Die Anordnung des Katalogs entspricht dem Aufbau des Textes. Die Farbbestimmung der keramischen Funde erfolgte nach Munsell, Soil Color Charts (New York, Edition 1992), die Tonbeschreibungen basieren, wenn nicht anders angeführt, auf makroskopischen Beobachtungen. Die Farbbestimmung für Glasfunde folgt H. Küppers, DuMont's Farbenatlas ${ }^{7}($ Köln 1995).

\section{Kat. 1}

Gattung: ESB; Form: Teller Atlante 60

Inv. 2005/63/4, FO: NT 05, SE 35

Erh.: 1 Rfrgt.

Ton: hart, fein, feinporös; Glimmer, sehr fein, mittel; weiße

Einschlüsse, fein, vereinzelt

Farbe Kern: 10YR4/4, Farbe Rand: 10YR4/4

Maße: RDm $26 \mathrm{~cm}$

Sek. Ofl.: flächig versintert

Überzug flächig, Farbe innen: 5YR6/4, Farbe außen: 5YR6/4.

Dat.: 1./2. Jh. n. Chr. (bis severisch)

\section{Kat. 2}

Abb. 13

Gattung: ESB; Form: Teller Atlante 60

Inv. 2005/58/2, FO: NT 05, SE 35

Bemerkungen: Bodeninnenseite mit 4 konzentrischen Rillen, violettes, stark glimmriges Fabric, orange Überzug

Erh.: 1 Bfrgt.

Ton: hart, sehr fein, feinporös; Glimmer, sehr fein, häufig

Farbe Kern: 2.5YR4/4, Farbe Rand: 2.5YR4/4

Maße: erh. L ca. $5 \mathrm{~cm}$

Sek. Ofl.: versintert, verbrannt

Überzug flächig, Farbe innen: 2.5YR6/8, Farbe außen: 2.5YR6/8, matt glänzend, kompakt

Dat.: 2. Jh. n. Chr.

\section{Kat. 3}

Abb. 13

Gattung: ESB; Form: Atlante 68/58

Inv. 2005/114/10, FO: NT 05, SE 35

Bemerkungen: Atlante 68 oder 58; mit Stempel (Stern) auf der Bodeninnenseite

Erh.: 1 Bfrgt.

Ton: mittelhart, fein, feinporös; Glimmer, fein, mittel

Farbe Kern: 2.5YR6/6, Farbe Rand: 2.5YR6/6

Maße: BDm 11,6 cm

Sek. Ofl.: versintert

Überzug flächig, Farbe innen: 2.5YR6/8, Farbe außen: 2.5YR6/8, matt glänzend, teilweise abgerieben Dat.: 1./2. Jh. n. Chr.

\section{Kat. 4}

Gattung: ESC; Form: Teller Atlante L26B

Inv. 2005/58/1, FO: NT 05, SE 35

Bemerkungen: 2 Fragmente, nicht anpassend

Erh.: 1 Rfrgt.

Ton: hart, fein, feinporös; weiße Einschlüsse, fein, mittel

Farbe Kern: 10R5/6, Farbe Rand: 10R5/6

Maße: RDm $18 \mathrm{~cm}$
Sek. Ofl.: versintert

Überzug, flächig, Farbe innen: 2.5YR6/8, Farbe außen: 2.5YR6/8, matt, abgerieben

Datierung: Mitte 1.- Mitte 2. Jh. n. Chr.

Kat. 5

Abb. 13

Gattung: ESC; Form: Schale Atlante L9

Inv. 2005/58/6, FO: NT 05, SE 35

Bemerkungen: breiter Rand, geschwungene Wand, Fabric: vereinzelt Glimmereinschlüsse

Erh.: 1 Rfrgt.

Ton: hart, fein, feinporös; Glimmer, fein, vereinzelt; weiße Einschlüsse, fein bis mittel, mittel

Farbe Kern: 2.5YR4/6, Farbe Rand: 2.5YR4/6

Maße: RDm $16 \mathrm{~cm}$

Sek. Ofl.: versintert

Überzug, flächig, Farbe innen: 2.5YR5/6, Farbe außen: 2.5YR5/6, matt, abgerieben

Dat.: spätes 1./2. Jh. n. Chr.

Kat. 6

Abb. 13

Gattung: ESC; Form: Teller Atlante L6

Inv. 2005/111/1, FO: NT 05, SE 35

Bemerkungen: Teller mit leicht eingebogenem Rand; grob, weiße und vulkanische Einschlüsse: Çandarlı; Überzug außen mit schwarzen Streifen (5YR3/1)

Erh.: 1 Rfrgt.

Ton: hart, mittelfein, mittelporös; schwarze Einschlüsse, graue Einschlüsse, weiße Einschlüsse: fein, häufig; graue Einschlüsse, mittel, mittel; weiße Einschlüsse, mittel, vereinzelt; Quarz, groß, vereinzelt (Polarisationsmikroskop, Vergrößerung: 40fach)

Farbe Kern: 2.5YR5/6, Farbe Rand: 2.5YR5/6

Maße: RDm $26 \mathrm{~cm}$

Überzug innen, Farbe innen: 10R4/8, Farbe außen: 5YR4/2, matt, kompakt

Dat.: Mitte - Ende 1. Jh. n. Chr.

Kat. 7

Abb. 13

Gattung: Feinware; Form: Becher

Inv. 2005/63/1, FO: NT 05, SE 35

Bemerkungen: mit Trichterrand

Erh.: 1 Rfrgt.

Ton: hart, fein, feinporös; weiße Einschlüsse, fein, mittel

Farbe Kern: 2.5YR4/6, Farbe Rand: 2.5YR4/6

Maße: RDm $10 \mathrm{~cm}$

Überzug flächig, Farbe innen: 2.5YR6/6, Farbe außen: 7.5YR6/3, matt, kompakt

Dat.: 2./3. Jh. n. Chr. 


\section{Kat. 8}

Gattung: Feinware; Form: Becher

Inv. 2005/60/1, FO: NT 05, SE 35

Bemerkungen: mit Trichterrand

Erh.: 1 Rfrgt.

Ton: hart, fein, feinporös; Glimmer, fein, mittel; graue Einschlüsse, fein, vereinzelt

Farbe Kern: 5YR5/6, Farbe Rand: 5YR5/6

Maße: RDm $9 \mathrm{~cm}$

Farbe innen: 5YR6/6, Farbe außen: 5YR6/6

Dat.: 2./3. Jh. n. Chr.

\section{Kat. 9}

Abb. 13

Gattung: Feinware; Form: Becher

Inv. 2005/60/2, FO: NT 05, SE 35

Bemerkungen: mit Trichterrand

Erh.: 1 Rfrgt.

Ton: hart, fein, feinporös; Glimmer, fein, mittel; helle Einschlüsse, fein, vereinzelt

Farbe Kern: 2.5YR4/6, Farbe Rand: 2.5YR4/6

Maße: RDm $9 \mathrm{~cm}$

Überzug außen, Farbe innen: 2.5YR5/6, Farbe außen:

7.5YR6/6, matt, teilweise abgerieben

Dat.: 2./3. Jh. n. Chr.

\section{Kat. 10}

Gattung: Amphore; Form: Peacock - Williams 45

Abb. 13

Inv. 2005/60/5, FO: NT 05, SE 35

Bemerkungen: frühes Rfrgt. (Absatz außen)

Erh.: 1 Rfrgt.

Ton: hart, fein, feinporös; Glimmer, fein, mittel; helle Einschlüsse, fein, vereinzelt

Farbe Kern: 5YR4/4, Farbe Rand: 5YR4/4

Maße: RDm 4 cm

Sek. Ofl.: -

Überzug flächig, Farbe innen: 5YR3/1, Farbe außen: 5YR3/1, matt

Dat.: 1./2. Jh. n. Chr.

\section{Kat. 11}

Abb. 13

Gattung: Amphore; Form: Peacock - Williams 45 Inv. 2005/63/5, FO: NT 05, SE 35

Bemerkungen: geöffneter Knauf

Erh.: 1 Knauf

Ton: hart, fein, feinporös; Glimmer, fein, viel; weiße Einschlüsse, fein, mittel

Farbe Kern: 2.5Y4/1, Farbe Rand: 2.5Y4/1

Maße: BDm $5 \mathrm{~cm}$

Sek. Ofl.: -

Überzug flächig, Farbe innen: 7.5YR5/3, Farbe außen: 7.5YR3/4, gefleckt mit 7.5YR2.5/1, matt, teilweise abgerieben

Dat.: 1./2. Jh. n. Chr.

\section{Kat. 12}

Abb. 13

Gattung: Amphore; Form: Peacock - Williams 45 Inv. 2005/111/5, FO: NT 05, SE 35
Bemerkungen: geöffneter, breiter Knauf (Standring)

Erh.: 1 Knauf

Ton: hart, fein, kompakt; Glimmer, fein, viel; weiße Einschlüsse, sehr fein, vereinzelt

Farbe Kern: 7.5YR7/4, Farbe Rand: 5YR6/6

Maße: BDm $5 \mathrm{~cm}$

Sek. Ofl.: flächig versintert

flächig, Farbe außen: 7.5YR7/3

Dat.: 2. Hälfte 1. Jh. v. Chr. - 1. Jh. n. Chr.

\section{Kat. 13}

Abb. 13

Gattung: Lampe; Typ: ROW

Inv. 2005/111/7, FO: NT 05, SE 35

Bemerkungen: gerillter Übergang zum Diskus

Erh.: 1 Schulter und Diskus

Ton: hart, fein, feinporös; Glimmer, fein, mittel; weiße Einschlüsse, fein bis mittel, selten

Farbe Kern: 7.5YR4/1, Farbe Rand: 7.5YR4/1

Maße: erh. L $4 \mathrm{~cm}$; erh. H $3 \mathrm{~cm}$

Sek. Ofl.: -

Farbe innen: 2.5YR5/6, Farbe außen: 2.5YR5/6

Dat.: 1.-3. Jh. n. Chr.

\section{Kat. 14}

Abb. 13

Gattung: Lampe; Typ: ROW

Inv. 2005/58/11, FO: NT 05, SE 35

Bemerkungen: mit Ringhenkel, total versintert bzw. mit Kalkmörtel aufgefüllt, zugehöriges Bodenfragment

Erh.: Schulter und Diskus

Ton: hart, fein, feinporös; Glimmer, fein, mittel; Quarz, mittel, mittel

Farbe Kern: 10R5/6, Farbe Rand: 10R5/6

Maße: RDm $4 \mathrm{~cm}$

Sek. Ofl.: versintert

Überzug außen, Farbe innen: 2.5YR4/8, Farbe außen: 2.5YR4/8, matt glänzend, teilweise abgerieben

Dat.: 1.-3. Jh. n. Chr.

\section{Kat. 15}

Abb. 14

Gattung: Küchenware; Form: Schüssel

Inv. 2005/109/1, FO: NT 05, SE 33

Bemerkungen: gröberes Fabric, große Glimmerplättchen; ausgestellte Lippe (abgebrochen)

Erh.: 1 Rfrgt.

Ton: sehr hart, mittelfein, feinporös; Glimmer, mittel, häufig; weiße Einschlüsse, fein, mittel; graue Einschlüsse, fein, mittel; Quarz, fein bis mittel, vereinzelt

Farbe Kern: 10R4/4, Farbe Rand: 2.5YR4/8

Maße: RDm $23 \mathrm{~cm}$

Sek. Ofl.: -

Farbe innen: 2.5YR5/6, Farbe außen: 2.5YR5/6

Dat.: hellenistisch

Kat. 16

Abb. 14

Gattung: Glas; Form: Schale

Inv. 2005/109/G3, FO: NT 05, SE 33 (leichte Fundvermischung mit SE 35) 
Bemerkungen: über Boden aufgelegter, dünner Glasfaden Erh.: 1 Bfrgt.

Farbe: S00/M00/C00

Maße: BDm $6 \mathrm{~cm}$

Sek. Ofl.: weiße Patina, haftend

Dat.: 1.-3. Jh. n. Chr.

\section{Kat. 17}

Abb. 14

Gattung: Glas; Form: Becher Inv. 2005/109/G3, FO: NT 05, SE 33 (leichte Fundvermischung mit SE 35)

Bemerkungen: mit leicht verdicktem Rand; Luftbläschen, mittel

Erh.: 1 Rfrgt.

Farbe: S00/C10/Y10

Maße: RDm $8 \mathrm{~cm}$

Dat.: 1. -3 . Jh. n. Chr.

\section{Kat. 18}

Abb. 14

Gattung: Küchenware; Form: Kasserolle

Inv. 2005/36/1, FO: NT 05, SE 22

Bemerkungen: mit breitem ausgebogenem Rand, Deckelfalz und gerippter Wand

Erh.: 1 Rfrgt.

Ton: hart, mittelfein, feinporös; Glimmer, mittel, mittel; gelbe Einschlüsse, fein, vereinzelt; weiße Einschlüsse, mittel, vereinzelt

Farbe Kern: 7.5YR3/2, Farbe Rand: 7.5YR3/2

Maße: RDm $24 \mathrm{~cm}$

Farbe innen: 5YR4/1, Farbe außen: 5YR4/1,

Dat.: 4./5. Jh. n. Chr.

\section{Kat. 19}

Abb. 14. 25

Gattung: Sonstiges; Form: Gusstiegel

Inv. 2005/36/2, FO: NT 05, SE 22

Bemerkungen: Pfanne/Gusstiegel mit unregelmäßigem Rand Erh.: zur Hälfte erhalten

Ton: hart, grob, feinporös; Glimmer, mittel, häufig; weiße Einschlüsse, grob, häufig

Farbe Kern: 2.5YR5/8, Farbe Rand: 5YR3/4

Maße: RDm $16 \mathrm{~cm}, \mathrm{BDm} 5,5 \mathrm{~cm}$

Farbe innen: 2.5YR5/6, Farbe außen: 5YR5/6

Dat.: -

\section{Kat. 20}

Abb. 14

Gattung: Amphore; Form: LRA 3

Inv. 2005/108/1, FO: NT 05, SE 22

Bemerkungen: halb geschlossener Knauf

Erh.: 1 Knauf

Ton: hart, fein, feinporös; Glimmer, fein, häufig; weiße Einschlüsse, fein, vereinzelt

Farbe Kern: 10YR4/1, Farbe Rand: 10R4/6

Maße: BDm 3,2 cm

Farbe innen: 2.5YR5/6, Farbe außen: 2.5YR5/6

Dat.: 4./5. Jh. n. Chr.

\section{Kat. 21}

Abb. 14. 25

Gattung: Lampe; Form: Doppelschnauzenlampe

Inv. 2005/36/3, FO: NT 05, SE 22

Bemerkungen: Doppelschnauzenlampe, 1 zugehöriges, aber nicht anpassendes Fragment

Erh.: 1 Schulter und Diskus

Ton: Glimmer, fein, mittel; dunkelgraue Einschlüsse, fein, vereinzelt

Farbe Kern: 5YR5/6, Farbe Rand: 5YR5/6

Maße: erh. H 2,8 cm; erh. B 4,4 cm

Farbe innen: 5YR7/6, Farbe außen: 5YR7/6

Dat.: 5. Jh. n. Chr.

\section{Kat. 22}

Abb. 14. 25

Gattung: Glas; Form: Becher Isings 106c/109

Inv. 2005/36/10 (G2), FO: NT 05, SE 22

Bemerkungen: Inv. G2; mit blauem Tropfendekor; S00/C10/ Y10 mit Tropfen Y10/M60/C80

Erh.: 1 Wfrgt.

Maße: $4 \times 2 \mathrm{~cm}$

Dat.: 4.-6. Jh. n. Chr.

\section{Kat. 23}

Abb. 14

Gattung: ESB; Form: Teller Atlante 14

Inv. 2005/106/1, FO: NT 05, SE 32

Erh.: 1 Rfrgt.

Ton: hart, fein, feinporös; Glimmer, fein, mittel; weiße Einschlüsse, fein bis mittel, vereinzelt

Farbe Kern: 5YR5/6, Farbe Rand: 5YR5/6

Maße: RDm $9 \mathrm{~cm}$

Überzug flächig, Farbe innen: 2.5YR5/6, Farbe außen: 2.5YR5/6, matt glänzend, kompakt

Dat.: 1. Jh. n. Chr.

\section{Kat. 24}

Abb. 14

Gattung: ESB; Form: Teller Atlante 58

Inv. 2005/105/1, FO: NT 05, SE 32

Erh.: 1 Wfrgt.

Ton: mittelhart, sehr fein, feinporös; mit freiem Auge keine Einschlüsse erkennbar

Farbe Kern: 5YR5/6, Farbe Rand: 5YR5/6

Maße: Dm Wandabsatz ca. $12 \mathrm{~cm}$

Überzug flächig, Farbe innen: 10R5/8, Farbe außen: 10R5/8, glänzend, abgerieben

Dat.: 2. Hälfte 1.-1. Hälfte 2. Jh. n. Chr.

\section{Kat. 25}

Abb. 14

Gattung: Feinware; Form: Becher

Inv. 2005/105/2, FO: NT 05, SE 32

Bemerkungen: Becher mit Trichterrand

Erh.: 1 Rfrgt.

Ton: hart, sehr fein, feinporös; weiße Einschlüsse, fein, vereinzelt

Farbe Kern: 5YR4/1, Farbe Rand: 5YR5/6

Maße: RDm $12 \mathrm{~cm}$

Farbe innen: 5YR5/6, Farbe außen: 10YR5/2

Dat.: 2./3. Jh. n. Chr. 


\section{Kat. 26}

Gattung: Küchenware; Form: Topf

Inv. 2005/106/3, FO: NT 05, SE 32

Bemerkungen: 2 zugehörige, nicht anpassende Wfrgte.

Erh.: 1 Rfrgt.

Ton: hart, fein, feinporös; weiße Einschlüsse, fein, mittel; wei-

ße Einschlüsse, mittel, vereinzelt; Glimmer, fein, vereinzelt

Farbe Kern: 7.5YR4/4, Farbe Rand: 7.5YR4/4

Maße: RDm $15 \mathrm{~cm}$

Farbe innen: 10YR4/1, Farbe außen: 10YR4/1

Dat.: Ende 4./1. Hälfte. 5. Jh. n. Chr.

\section{Kat. 27}

Gattung: Küchenware; Form: Topf/Kasserolle Inv. 2005/52/2, FO: NT 05, SE 32

Bemerkungen: total versintert; ausgebogener Rand, gerippte Wand

Erh.: 1 Rfrgt.

Ton: hart, fein, feinporös; weiße Einschlüsse, fein, selten; rötliche Einschlüsse, fein, selten; graue Einschlüsse, fein, selten Farbe Kern: 2.5YR4/1, Farbe Rand: 2.5YR4/1

Maße: RDm $18 \mathrm{~cm}$

Sek. Ofl.: flächig versintert

Farbe innen: 2.5YR5/4, Farbe außen: 2.5YR5/4

Dat.: 4./5. Jh. n. Chr.

\section{Kat. 28}

Abb. 15

Gattung: Küchenware; Form: Topf

Inv. 2005/52/1, FO: NT 05, SE 32

Bemerkungen: Topf/Schüssel mit ausgebogenem Rand und Deckelfalz; spätantikes Fabric

Erh.: 1 Rfrgt.

Ton: mittelhart, grob, mittelporös; schwarze Einschlüsse, grob, vereinzelt; weißgelbliche Einschlüsse, grob, vereinzelt; weiße Einschlüsse, klein, viel

Farbe Kern: 5YR3/4, Farbe Rand: 5YR3/4

Maße: RDm $24 \mathrm{~cm}$

Farbe innen: GLEY1 3/N, Farbe außen: GLEY1 3/N

Dat.: 4./5. Jh. n. Chr.

Kat. 29

Abb. 15

Gattung: Amphore; Form: LRA 3

Inv. 2005/105/6, FO: NT 05, SE 32

Bemerkungen: nicht ganz geschlossener Knauf

Erh.: 1 Knauf

Ton: hart, fein, feinporös; Glimmer, fein, häufig; weiße Einschlüsse, fein, vereinzelt

Farbe Kern: 10YR4/1, Farbe Rand: 10YR4/1

Maße: BDm $4 \mathrm{~cm}$

Farbe innen: 2.5YR5/6, Farbe außen: 2.5YR5/6

Dat.: 4./5. Jh. n. Chr.

\section{Kat. 30}

Abb. 15

Gattung: Glas; Form: Becher/Schale

Inv. NT05/52/G1, FO: NT 05, SE 32

Bemerkungen: massiver Standring, feine Bearbeitungsspuren

Maße: BDm $7 \mathrm{~cm}$

Farbe: Y30/M00/C20

Dat.: 3./4. Jh. n. Chr. (?)
Kat. 31

Abb. 15

Gattung: Glas; Form: Becher/Schale

Inv. NT05/106/G1, FO: NT 05, SE 32

Bemerkungen: massiver, kleiner Standring

Maße: BDm $3 \mathrm{~cm}$

Sek. Ofl.: schwarze Punkte (Patina)

Farbe: S10/C00/Y50

Dat.: 3./4. Jh. n. Chr. (?)

Kat. 32

Abb. 15

Gattung: Glas; Form: Becher/Schale Inv. NT05/106/G2, FO: NT 05, SE 32

Bemerkungen: massiver, kleiner Standring

Maße: BDm 4,4 cm

Sek. Ofl.: dunkle Patina

Farbe: Y10/M00/C10

Dat.: 3./4. Jh. n. Chr. (?)

Kat. 33

Abb. 15

Gattung: ARS; Form: Platte Hayes 67

Inv. 2005/30/1, FO: NT 05, SE 17

Bemerkungen: wohl Gruppe 1: 360-420 n. Chr.

Erh.: 1 Rfrgt.

Ton: hart, fein, fein bis mittelporös, Glimmer, fein, vereinzelt; weiße Einschlüsse, fein bis mittel, vereinzelt

Farbe Kern: 10R6/8, Farbe R: 10R6/8

Maße: RDm $44 \mathrm{~cm}$

Überzug flächig, Farbe innen: 10R6/8, Farbe außen: 2.5YR6/8, matt glänzend, kompakt

Dat.: $360-470$ n. Chr.

Kat. 34

Abb. 15

Gattung: Amphore

Inv. 2005/30/9, FO: NT 05, SE 17

Bemerkungen: mit verdicktem Rand und an der Oberseite geripptem, an der Unterseite unregelmäßig gekehltem Henkel; wohl Import aus Schwarzmeerraum; innen am Rand abrinnender mattheller Überzug

Erh.: 1 Rfrgt.

Ton: hart, fein, mittelporös; Glimmer, fein, vereinzelt; weiße Einschlüsse, fein, mittel; schwarze Einschlüsse, fein, mittel Farbe Kern: 2.5YR6/8, Farbe Rand: 2.5YR6/8

Maße: RDm 8,8 cm

Überzug, partiell, Farbe innen: 10R6/8, Farbe außen: 10R6/6, matt

Dat.: -

Kat. 35

Abb. 16

Gattung: Amphore; Form: LRA 3

Inv. 2005/44/3, FO: NT 05, SE 17

Bemerkungen: noch leicht geöffneter Knauf

Erh.: 1 Knauf

Ton: mittelhart, fein, feinporös; Glimmer, fein, viel; weiße Einschlüsse, fein, vereinzelt

Farbe Kern: 10R4/6, Farbe Rand: 10R4/6

Maße: BDm 4,2 cm,

Farbe innen: 10R4/4, Farbe außen: 10R4/4 
Sek. Ofl.: versintert

Dat.: 4./5. Jh. n. Chr.

\section{Kat. 36}

Gattung: Amphore; Form: LRA 3

Inv. 2005/30/11, FO: NT 05, SE 17

Bemerkungen: noch leicht geöffneter Knauf

Erh.: 1 Knauf

Ton: hart, fein, feinporös; Glimmer, fein, viel; weiße Einschlüsse, fein, vereinzelt; schwarze Einschlüsse, fein, vereinzelt

Farbe Kern: 2.5YR3/6, Farbe Rand: 2.5YR3/6

Maße: BDm 2,6 cm

Farbe innen: 2.5YR5/6, Farbe außen: 2.5YR6/6

Sek. Ofl.: versintert

Dat.: 4./5. Jh. n. Chr.

\section{Kat. 37}

Gattung: Küchenware; Form: Kasserolle/Topf

Inv. 2005/30/6, FO: NT 05, SE 17

Bemerkungen: mit nach innen abfallendem, profiliertem Rand und gerippter Wand

Erh.: 1 Rfrgt.

Ton: mittelhart, fein bis mittelfein, feinporös; Glimmer, fein, viel; weiße Einschlüsse, fein bis mittel, vereinzelt

Farbe Kern: 5YR4/6, Farbe Rand: 10YR4/2

Maße: RDm $17 \mathrm{~cm}$

Farbe innen: 2.5YR7/4, Farbe außen: 75YR5/3-4/1

Sek. Ofl.: innen stark versintert

Dat.: 4./5. Jh. n. Chr.

\section{Kat. 38}

Abb. 16

Gattung: Küchenware; Form: Topf

Inv. 2005/30/7, FO: NT 05, SE 17

Bemerkungen: mit ausgebogenem abgerundetem Rand und Deckelfalz

Erh.: 1 Rfrgt.

Ton: hart, fein bis mittelfein, mittelporös; Glimmer, sehr fein, vereinzelt; weiße Einschlüsse, fein, vereinzelt

Farbe Kern: 10YR3/2, Farbe Rand: 10YR3/2

Maße: RDm $22 \mathrm{~cm}$

Farbe innen: GLEY1 4/N-3/N, Farbe außen: GLEY1 4/N-3/N

Dat.: 4./5. Jh. n. Chr.

\section{Kat. 39}

Abb. 16

Gattung: Lampe

Inv. 2005/30/16, FO: NT 05, SE 17

Bemerkungen: Loch schlampig ausgestochen, sodass Füllung noch steckt; wohl lokale Produktion

Erh.: 1 Henkel

Ton: hart, fein, feinporös; Glimmer, fein, vereinzelt; weiße Einschlüsse, fein, vereinzelt

Farbe Kern: 7.5YR3/1, Farbe Rand: 7.5YR6/6

Maße: erh. H 3,2 cm; erh. B ca. $3 \mathrm{~cm}$

Farbe innen: 7.5YR7/4, Farbe außen: 7.5YR7/4

Dat.: spätantik

\section{Kat. 40}

Abb. 16

Gattung: Amphore; Form: LRA 3

Inv. 2005/34/1, FO: NT 05, SE 20

Bemerkungen: Knauf abgebrochen, fast geschlossen

Erh.: 1 Knauf

Ton: hart, fein, feinporös; Glimmer, fein, viel; helle Einschlüsse, fein, vereinzelt

Farbe Kern: 2.5YR3/6, Farbe Rand: $2.5 Y R 3 / 6$

Maße: BDm ca. $3,8 \mathrm{~cm}$

Farbe innen: 5YR5/3, Farbe außen: 2.5YR5/6

Sek. Ofl.: flächig versintert

Dat.: 4./5. Jh. n. Chr.

Kat. 41

Abb. 16

Gattung: Küchenware; Form: Deckel

Inv. 2005/34/2, FO: NT 05, SE 20

Bemerkungen: mit verdicktem Rand

Erh.: 1 Rfrgt.

Ton: hart, fein, feinporös; Glimmer, fein, viel; helle Einschlüsse, fein bis mittel, mittel; dunkle Einschlüsse, fein, vereinzelt Farbe Kern: 5YR3/4, Farbe Rand: 5YR3/4

Maße: RDm 12,6 cm

Farbe innen: 5YR5/4, Farbe außen: 2.5YR3/3

Sek. Ofl.: partiell versintert

Dat.: spätantik

Kat. 42

Abb. 16. 25

Gattung: Glas; Form: (Arm-)Ring

Inv. NT05/100/G5

Bemerkungen: Luftbläschen, fein, vereinzelt

Farbe: Y99/M80/C70 (blaubraun)

Maße: Dm max. 4,2 cm

Sek. Ofl.: weiße Patina

Dat.: -

Kat. 43

Abb. 16

Gattung: Glas; Form: Becher

Inv. 2005/107/G1, FO: NT 05, SE 26 (Putzen auf SE 22, Reste der SE 26)

Bemerkungen: Röhrchenstandring eines Kelchglases

Farbe: $\mathrm{S} 00 / \mathrm{C} 00 / \mathrm{Y} 00$

Erh.: 1 Bfrgt.

Maße: BDm 5,2 cm

Dat.: spätantik

Kat. 44

Gattung: Küchenware; Form: Topf

Inv. 2005/47/1, FO: NT 05, SE 28

Bemerkungen: mit ausgebogenem, innen deutlich abgesetztem Rand und gerippter Wand

Erh.: 1 Rfrgt.

Ton: hart, fein, feinporös; Glimmer, fein, viel; weiße Einschlüsse, fein, vereinzelt

Farbe Kern: 2.5YR4/4, Farbe Rand: 2.5YR4/4

Maße: RDm $18 \mathrm{~cm}$

Farbe innen: GLEY1 3/N, Farbe außen: GLEY1 3/N

Dat.: Ende 4./Anfang 5. Jh. n. Chr. 


\section{Kat. 45}

Abb. 16

Gattung: Amphore

Inv. 2005/47/2, FO: NT 05, SE 28

Bemerkungen: mit innen und außen verdicktem Rand, mattheller, kompakter Überzug

Erh.: 1 Rfrgt.

Ton: hart, fein, feinporös; Glimmer, fein, vereinzelt; weiße Einschlüsse, fein, vereinzelt; dunkle Einschlüsse, fein, vereinzelt; rote Einschlüsse, fein, vereinzelt

Farbe Kern: 2.5YR7/6, Farbe Rand: 10R7/4

Maße: RDm $12 \mathrm{~cm}$

Überzug, flächig, Farbe innen: 2.5Y8/2, Farbe außen: 2.5Y8/2, matt, kompakt

Dat.: -

Kat. 46

Abb. 16

Gattung: Lampe

Inv. 2005/47/7, FO: NT 05, SE 28

Bemerkungen: mit Kerbdekor auf Schulter

$\mathrm{Fi} / \mathrm{Fa} / \mathrm{FB}$ nicht mehr erkennbar

Erh.: 1 Schulter

Ton: mittelhart, fein, feinporös; Glimmer, fein, viel

Farbe Kern: -, Farbe Rand:-

Maße: Dm Diskus 3,2 cm

Sek. Ofl.: verbrannt

Dat.: spätantik

\section{Kat. 47}

Abb. 16

Gattung: Lampe; Form: Doppelschnauzenlampe

Inv. 2005/101/3, FO: NT 05, SE 28

Bemerkungen: gelochter Lampenhenkel, wohl von mehrschnauziger Lampe

Erh.: 1 Henkel

Ton: mittelhart, fein, feinporös; Glimmer, fein, viel

Farbe Kern: 2.5YR5/6, Farbe Rand: 2.5YR5/6

Maße: erh. H ca. $2 \mathrm{~cm}$

Sek. Ofl:: abgerieben

Farbe innen: 2.5YR6/6, Farbe außen: 2.5YR6/6

Dat.: 5. Jh. n. Chr

\section{Kat. 48}

Abb. 16

Gattung: Glas; Form: Becher/Schale

Inv. 2005/47/4, FO: NT 05, SE 28

Bemerkungen: Röhrchenstandring, 2 zusammengehörige, aber nicht anpassende Fragmente

Erh.: 1 Bfrgt.

Farbe: S00/C10/Y10

Maße: BDm 4,6 cm

Dat:: spätantik

\section{Kat. 49}

Abb. 17

Gattung: Küchenware; Form: Topf

Inv. 2005/103/1, FO: NT 05, SE 31

Bemerkungen: mit ausgebogenem, innen deutlich abgesetztem

Rand und gerippter Wand

Erh.: 1 Rfrgt.

Ton: hart, fein, feinporös; Glimmer, vereinzelt, fein
Farbe Kern: 5YR3/2, Farbe Rand: 10R4/6

Maße: RDm $17 \mathrm{~cm}$

Sek. Ofl.: versintert

Farbe innen: 7.5YR3/1, Farbe außen: 7.5YR3/1

Dat.: Ende 4./5. Jh. n. Chr.

Kat. 50

Abb. 17

Gattung: Küchenware; Form: Topf

Inv. 2005/104/1, FO: NT 05, SE 31

Bemerkungen: mit ausgebogenem, innen deutlich abgesetztem

Rand und gerippter Wand

Erh.: 1 Rfrgt.

Ton: sehr hart, fein, feinporös; Glimmer, fein, mittel; helle Einschlüsse, fein, vereinzelt; dunkle Einschlüsse, fein, vereinzelt Farbe Kern: 7.5YR4/2, Farbe Rand: 2.5YR3/6

Maße: RDm 19,4 cm

Farbe innen: 2.5YR4/4, Farbe außen: 5YR4/2

Dat.: Ende 4./Anfang 5. Jh. n. Chr.

Kat. 51

Abb. 17

Gattung: Lampe

Inv. 2005/104/3, FO: NT 05, SE 31

Bemerkungen: gelochter Lampengriff mit noch schwach sichtbarem Tannenreisdekor; Reste eines matthellen Überzugs

Erh.: 1 Henkel

Ton: weich, fein, feinporös; Glimmer, fein, mittel; helle Einschlüsse, fein, mittel; dunkle Einschlüsse, fein, vereinzelt

Farbe Kern: 5YR6/6, Farbe Rand: 5YR6/6

Maße: erh. $\mathrm{H} 3 \mathrm{~cm}$

Farbe innen: 5YR6/6, Farbe außen: 5YR6/6

Dat.: spätantik

Kat. 52

Abb. 17

Form: Becher/Schale

Inv. NT05/104/G2

Bemerkungen: kleiner, massiver Standring, vereinzelt feine Luftbläschen

Erh.: 1 Bfrgt.

Maße: BDm 2,8 cm

Farbe: Y20/M30/C90 (Standring)

Dat.: 3./4. Jh. n. Chr.

Kat. 53

Abb. 17

Form: Becher/Schale

Inv. NT05/104/G3

Bemerkungen: an der Ofl. vereinzelte Luftbläschen

Erh.: 1 Bfrgt.

Maße: BDm $7 \mathrm{~cm}$

Farbe: Y10/M00/C10

Dat: $3 . / 4$. Jh. n. Chr.

Kat. 54

Abb. 17

Gattung: LRC; Form: Teller Hayes 4

Inv. 2005/35/1, FO: NT 05, SE 21 (Grube)

Bemerkungen: vgl. Ladstätter 2005, 79; teilweise verbrannt, Wandknick im oberen Gefäßdrittel; sehr groß 
Erh.: 1 Rfrgt.

Ton: hart, fein, feinporös; Glimmer, fein, vereinzelt; weiße Einschlüsse, fein, vereinzelt

Farbe Kern: 10R5/6, Farbe Rand: 10R5/6

Maße: RDm $34 \mathrm{~cm}$

Sek. Ofl.: verbrannt

Überzug, flächig, Farbe innen: 10R5/6, Farbe außen: 10R5/6, matt, kompakt

Dat.: 5. Jh. n. Chr.

\section{Kat. 55}

Abb. 17

Gattung: Lampe; Form: Doppelschnauzenlampe

Inv. 2005/35/3, FO: NT 05, SE 21 (Grube)

Bemerkungen: Doppelschnauzenlampe

Erh.: 1 Schulter und Diskus

Ton: hart, fein, feinporös; Glimmer, fein, vereinzelt; weiße Einschlüsse, fein, vereinzelt

Farbe Kern: 7.5YR5/6, Farbe Rand: 7.5YR5/4

Maße: erh. H ca. 1,4 cm; erh. B Diskus/Schulter 5,2 cm

Sek. Ofl.: -

Farbe innen: 5YR7/6, Farbe außen: 5YR7/6

Dat.: spätantik (5. Jh. n. Chr.)

\section{Kat. 56}

Abb. 17

Gattung: LRC; Form: Teller Hayes 3

Inv. 2005/80/1, FO: NT 05, SE 12

Bemerkungen: hoher Rand

Erh.: 1 Rfrgt.

Ton: hart, fein, feinporös; Glimmer, fein, vereinzelt; weiße Einschlüsse, fein, mittel

Farbe Kern: 2.5YR6/8, Farbe Rand: 2.5YR6/8

Maße: RDm $30 \mathrm{~cm}$

Überzug, flächig, Farbe innen: 10R5/8, Farbe außen: 10R5/8, matt, kompakt

Dat.: 6. Jh. n. Chr.

\section{Kat. 57}

Abb. 17

Gattung: ARS; Form: Teller Hayes 50B

Inv. 2005/19/1, FO: NT 05, SE 12

Bemerkungen: vgl. bes. Hayes 1972, Abb. 12, 56.

Erh.: 1 Bfrgt.

Ton: hart, fein, feinporös; weiße Einschlüsse, fein, vereinzelt; graue Einschlüsse, fein, vereinzelt

Farbe Kern: 10R6/8, Farbe Rand: 10R6/8

Maße: BDm $28 \mathrm{~cm}$

Überzug flächig, Farbe innen: 10R6/8, Farbe außen: 10R6/8, matt glänzend, kompakt

Dat.: $350-400$ n. Chr.

\section{Kat. 58}

Abb. 17

Gattung: Amphore; Form: Agora M 273/Samos Cistern Type Inv. 2005/99/4, FO: NT 05, SE 12

Bemerkungen: zwischen Samos Cistern Type und Agora M 273, Ofl. glimmrig, weiße Einschlüsse, teilweise versintert Erh.: 1 Rfrgt.

Ton: hart, fein, feinporös; Glimmer, fein bis mittel, mittel; weiße Einschlüsse, mittel, vereinzelt; schwarze Einschlüsse, fein, vereinzelt

Farbe Kern: 10R3/1, Farbe Rand: 2.5YR4/6

Maße: RDm $9 \mathrm{~cm}$

Sek. Ofl.: versintert

Farbe innen: 10R5/6, Farbe außen: 2.5YR5/6

Dat.: 5./6. Jh. n. Chr. (?)

\section{Kat. 59}

Abb. 17

Gattung: Amphore; Form: LRA 3

Inv. 2005/19/3, FO: NT 05, SE 12

Bemerkungen: nicht ganz geschlossen

Erh.: 1 Knauf

Ton: hart, fein, feinporös; Glimmer, fein, viel

Farbe Kern: 5YR4/6, Farbe Rand: 5YR4/6

Maße: BDm 2,8 cm

Farbe innen: 2.5YR6/6, Farbe außen: 2.5YR6/6

Dat.: 5. Jh. n. Chr.

Kat. 60

Gattung: Amphore; Form: LRA 3

Inv. 2005/19/4, FO: NT 05, SE 12

Bemerkungen: nicht ganz geschlossen

Erh.: 1 Knauf

Ton: hart, fein, feinporös; Glimmer, fein, viel

Farbe Kern: 2.5YR5/6, Farbe Rand: 2.5YR5/6

Maße: BDm $3 \mathrm{~cm}$

Farbe innen: 5YR5/6, Farbe außen: 5YR5/6

Dat.: 5. Jh. n. Chr.

Abb. 17

Kat. 61

Abb. 17

Gattung: Amphore; Form: LRA 3

Inv. 2005/19/9, FO: NT 05, SE 12

Erh.: 1 Rfrgt.

Ton: hart, fein, feinporös; Glimmer, fein, mittel; dunkle Einschlüsse, fein, vereinzelt

Farbe Kern: 5YR4/6, Farbe Rand: 5YR4/6

Maße: RDm 3,4 cm

Farbe innen: 2.5YR6/6, Farbe außen: 2.5YR6/4

Dat.: spätantik

\section{Kat. 62}

Abb. 18

Gattung: Amphore; Form: Agora M 273

Inv. 2005/19/8, FO: NT 05, SE 12

Bemerkungen: mit verdicktem Rand, matthelle Ofl., lokales Fabric

Erh.: 1 Rfrgt.

Ton: hart, fein, feinporös; Glimmer, fein, viel; Glimmer, groß, vereinzelt; schwarze Einschlüsse, fein, viel; schwarze Einschlüsse, mittel, vereinzelt; weiße Einschlüsse, mittel, mittel; weiße Einschlüsse, groß, vereinzelt; rötliche Einschlüsse, mittel, mittel; rötliche Einschlüsse, groß, vereinzelt (Polarisationsmikroskop, 4-fache Vergrößerung)

Farbe Kern: 10YR7/4, Farbe Rand: 10YR7/4

Maße: RDm 10,2 cm

Sek. Ofl.: versintert

Überzug, flächig, Farbe innen: 10YR7/4, Farbe außen: 10 YR $7 / 4$, matt, teilweise abgerieben

Dat.: 4./5. Jh. n. Chr. 


\section{Kat. 63}

Abb. 18

Gattung: Amphore

Inv. 2005/98/2, FO: NT 05, SE 12

Bemerkungen: mit hängender Lippe, Ofl. leicht glimmrig, Farbe ähnlich LRA 3, aber auch mittelgroße weiße und dunkle Einschlüsse

Erh.: 1 Rfrgt.

Ton: hart, fein, feinporös; Glimmer, fein, vereinzelt; weiße Einschlüsse, mittel, vereinzelt; dunkle Einschlüsse, fein, vereinzelt

Farbe Kern: 2.5YR4/6, Farbe Rand: 2.5YR4/6

Maße: RDm $12 \mathrm{~cm}$

Farbe innen: 10R5/6, Farbe außen: 10R5/6

Dat.: spätantik

\section{Kat. 64}

Abb. 18

Gattung: Lampe; Form: Doppelschnauzenlampe

Inv. 2005/99/1, FO: NT 05, SE 12

Bemerkungen: mit zentralem gelochtem Henkel, auf Schulter umlaufende Warzenreihe

Erh.: 1 Schulter und Diskus

Ton: hart, fein, feinporös; Glimmer, fein, vereinzelt; weiße Einschlüsse, fein, vereinzelt

Farbe Kern: 7.5YR6/6, Farbe Rand: 7.5YR6/6

Maße: erh. H 1,2 cm; erh. B (Schulter) ca. $6 \mathrm{~cm}$

Farbe innen: 5YR7/3, Farbe außen: 7.5YR7/3

Dat.: spätantik (5. Jh.)

\section{Kat. 65}

Abb. 18

Gattung: Küchenware; Form: Kasserolle

Inv. 2005/80/7, FO: NT 05, SE 12

Bemerkungen: mit innen abgestuftem Rand und gerippter Wand

Erh.: 1 Rfrgt.

Ton: sehr hart, mittelfein, mittelporös; Glimmer, fein, viel; weiße Einschlüsse, fein, vereinzelt

Farbe Kern: 5YR4/1, Farbe Rand: 5YR6/4

Maße: RDm $22 \mathrm{~cm}$

Farbe innen: 2.5YR4/4, Farbe außen: 2.5YR2.5/1

Dat.: 5. Jh. n. Chr.

\section{Kat. 66}

Abb. 18

Gattung: Küchenware; Form: Schüssel

Inv. 2005/80/8, FO: NT 05, SE 12

Bemerkungen: Schüssel/Pfanne mit knopfartig verdicktem

Rand

Erh.: 1 Rfrgt.

Ton: sehr hart, mittelfein, mittelporös; Glimmer, fein, viel; weiße Einschlüsse, fein, vereinzelt

Farbe Kern: 2.5YR4/1, Farbe Rand: 10R4/4

Maße: RDm $30 \mathrm{~cm}$

Sek. Ofl:: versintert

flächig, Farbe innen: GLEY1 3/N, Farbe außen: GLEY1 3/N

Dat.: 5. Jh. n. Chr.

Kat. 67

Abb. 18

Gattung: LRC; Form: Teller Hayes 3/10

Inv. 2005/10/4, FO: NT 05, SE 8
Erh.: 1 Rfrgt.

Ton: sehr hart, fein, feinporös; Glimmer, fein, vereinzelt; helle Einschlüsse, fein, viel

Farbe Kern: 2.5YR4/8, Farbe Rand: 2.5YR4/8

Maße: RDm $20 \mathrm{~cm}$

Überzug, flächig, Farbe innen: 5YR4/6, Farbe außen: 2.5YR5/6, matt, kompakt

Datierung: 6. Jh. n. Chr.

\section{Kat. 68}

Abb. 18

Gattung: LRC; Form: Teller Hayes 3/10

Inv. 2005/10/5, FO: NT 05, SE 8

Erh.: 1 Rfrgt.

Ton: hart, fein, feinporös; Glimmer, sehr fein, vereinzelt; dunkle Einschlüsse, sehr fein, vereinzelt

Farbe Kern: 2.5YR5/8, Farbe Rand: 2.5YR5/8

Maße: RDm $18 \mathrm{~cm}$

Überzug flächig, Farbe innen: 5YR4/6, Farbe außen: 2.5YR5/6, matt, teilweise abgerieben

Dat.: 6. Jh. n. Chr.

\section{Kat. 69}

Abb. 18

Gattung: ERSW; Form: Teller Hayes 3

Inv. 2005/10/3, FO: NT 05, SE 8

Erh.: 1 Ganzgefäß

Ton: hart, fein, feinporös; Glimmer, fein, vereinzelt; helle Einschlüsse, fein, vereinzelt

Farbe Kern: 7.5YR5/6, Farbe Rand: 7.5YR5/6

Maße: RDm $19 \mathrm{~cm}$

Überzug flächig, Farbe innen: 2.5YR4/8, Farbe außen: 2.5YR4/8, matt, teilweise abgerieben

Dat.: 6. Jh. n. Chr.

Kat. 70

Abb. 6

Gattung: ERSW; Form: Teller Hayes 3

Inv. 2005/10/6, FO: NT 05, SE 8

Bemerkungen: Bodeninnenseite abgebrochen

Erh.: 1 Bfrgt.

Ton: hart, fein, feinporös; Glimmer, fein, mittel

Farbe Kern: 2.5YR5/6, Farbe Rand: 5YR5/2

Maße: BDm 12,2 cm

Sek. Ofl.: abgesplittert

Überzug flächig, Farbe innen: 2.5YR5/2, Farbe außen: 7.5YR5/6, matt, abgerieben

Dat.: 5./6. Jh. n. Chr.

Kat. 71

Abb. 18. 25

Gattung: ERSW; Form: Teller Hayes 3

Inv. 2005/92/1, FO: NT 05, SE 8

Bemerkungen: mit Rest eines Stempels (Kreuz)

Erh.: 1 Bfrgt.

Ton: hart, fein, feinporös; Glimmer, sehr fein, mittel

Farbe Kern: 5YR5/4, Farbe Rand: 5YR5/4

Maße: BDm $8 \mathrm{~cm}$

Überzug flächig, Farbe innen: 10R5/8, Farbe außen: 10R5/8, matt, teilweise abgerieben

Dat.: 6. Jh. n. Chr. 
Kat. 72

Abb. 19. 25

Gattung: Mäandertalsigillata; Form: Schüssel/Teller

Inv. 2005/10/1, FO: NT 05, SE 8

Bemerkungen: Mäandertalsigillata, mit eingezogenem, innen abgesetztem Rand, außen verdickter facettierter Rundlippe; auf der Bodeninnenseite feines Punktrouletting

Erh.: 1 Rfrgt./1 Bfrgt.

Ton: hart, fein, feinporös; Glimmer, sehr fein, mittel; Glimmer/ transparente Einschlüsse, mittel, vereinzelt; helle Einschlüsse, fein bis mittel, mittel; dunkle Einschlüsse, mittel, vereinzelt (Polarisationsmikroskop, Vergrößerung: 40-fach)

Farbe Kern: 10YR5/3, Farbe Rand: 7.5YR6/6

Maße: RDm $36 \mathrm{~cm}$

Überzug flächig, Farbe innen: 10R4/6, Farbe außen: 10R4/6, matt glänzend, teilweise abgerieben

Dat.: spätantik/frühbyzantinisch

\section{Kat. 73}

Abb. 19

Gattung: Mäandertalsigillata; Form: Teller/ Schüssel

Inv. 2005/10/2, FO: NT 05, SE 8

Bemerkungen: Mäandertalsigillata, breiter, nach außen hin leicht abfallender, leicht gewellter Rand, dunkelrot überzogen Erh.: 1 Rfrgt. und 1 Wfrgt.

Ton: hart, fein, feinporös; Poren, mittel, mittel; Glimmer, sehr fein bis fein, vereinzelt; helle Einschlüsse, sehr fein bis fein, viel; dunkle Einschlüsse, fein, vereinzelt (Polarisationsmikroskop, Vergrößerung: 40-fach)

Farbe Kern: 10YR4/2, Farbe Rand: 5YR5/6

Maße: RDm $34 \mathrm{~cm}$

Überzug flächig, Farbe innen: 10R4/6, Farbe außen: 10R4/6, matt glänzend, kompakt

Dat.: spätantik/frühbyzantinisch

\section{Kat. 74}

Abb. 19

Gattung: Amphore; Form: LRA 1

Inv. 2005/10/8, FO: NT 05, SE 8

Bemerkungen: LRA 1 mit kantig abgestrichenem Rand, Brandspuren auf einer Seite des Randes

Erh.: 1 Rfrgt.

Ton: hart, fein, feinporös; helle Einschlüsse, fein, mittel; dunkle Einschlüsse, fein, mittel

Farbe Kern: 7.5YR6/6, Farbe Rand: 7.5YR6/6

Maße: RDm $11 \mathrm{~cm}$

Sek. Ofl.: partiell verbrannt

Farbe innen: 10YR7/2, Farbe außen: 10YR7/2

Dat.: 5. - Mitte 7. Jh. n. Chr.

Kat. 75

Abb. 19

Gattung: Amphore; Form: LRA 3

Inv. 2005/10/9, FO: NT 05, SE 8

Erh.: 1 Rfrgt.

Ton: hart, fein, feinporös; Glimmer, sehr fein, mittel

Farbe Kern: 2.5 YR $4 / 8$, Farbe Rand: 2.5 YR $4 / 8$

Maße: RDm 3,2 cm

\footnotetext{
${ }_{183}$ Lesung H. Taeuber.
}

Farbe innen: 2.5YR5/6, Farbe außen: 2.5YR5/6

Dat.: 4.-6. Jh. n. Chr.

Kat. 76

Abb. 19

Gattung: Amphore; Form: Ephesos 56 (?)

Inv. 2005/92/3, FO: NT 05, SE 8

Erh.: 1 Rfrgt.

Ton: hart, fein, feinporös; Glimmer, sehr fein, viel

Farbe Kern: 2.5YR4/6, Farbe Rand: 2.5YR4/6

Maße: RDm $3 \mathrm{~cm}$

Farbe innen: 10R4/6, Farbe außen: 10R4/6

Dat.: 5./6. Jh. n. Chr.

Kat. 77

Abb. 19. 25

Gattung: Amphore; Form: LRA 3

Inv. 2005/92/4, FO: NT 05, SE 8

Bemerkungen: fast geschlossener Knauf

Erh.: 1 Knauf

Ton: hart, fein, feinporös; Glimmer, sehr fein, vereinzelt

Farbe Kern: 2.5YR3/6, Farbe Rand: 2.5YR3/6

Maße: BDm $1 \mathrm{~cm}$

Sek. Ofl.: -

Farbe innen: 2.5 YR $4 / 4$, Farbe außen: 2.5YR4/4

Dat.: 4./5. Jh. n. Chr.

Kat. 78

Abb. 19. 25

Gattung: Amphore; Form: LRA 3

Inv. 2005/14/1, FO: NT 05, SE 8

Bemerkungen: Fabric ähnlich LRA 3 (etwas rauer), mit Graffito:

-e(?)nteton (Mitte Eta)

$-1(?)$ is e (= Eta) elipo-

-lis

(Abstand)

-eis ${ }^{183}$

Ton: hart, fein, porös; Glimmer, fein, mittel; helle Einschlüsse, fein, vereinzelt

Farbe Kern: 2.5YR4/6, Farbe Rand: 2.5YR4/6

Maße: erh. $\mathrm{H}$ ca. $7 \mathrm{~cm}$; erh. B ca. $5,4 \mathrm{~cm}$

Farbe innen: 2.5YR5/4, Farbe außen: 5YR4/3

Dat.: spätantik

Kat. 79

Abb. 19

Gattung: ARS; Form: Schale Hayes 91C

Inv. 2005/57/1, FO: NT 05, SE 34

Bemerkungen: Hayes 91, Variante

Erh.: 1 Rfrgt.

Ton: hart, fein, feinporös; weiße Einschlüsse, fein, vereinzelt;

Glimmer, fein, vereinzelt

Farbe Kern: 2.5YR6/8, Farbe Rand: 2.5YR6/8

Maße: RDm $10 \mathrm{~cm}$

Überzug, flächig, Farbe innen: 10R5/8, Farbe außen: 10R5/8, matt glänzend, kompakt

Dat.: 5./frühes 6. Jh. n. Chr. 
Kat. 80

Gattung: LRC; Form: Teller Hayes 2A/4

Abb. 19

Inv. 2005/57/2, FO: NT 05, SE 34

Erh.: 1 Bfrgt.

Ton: hart, fein, feinporös; weiße Einschlüsse, fein, mittel

Farbe Kern: 2.5YR6/8, Farbe Rand: 2.5YR6/8

Maße: BDm $10 \mathrm{~cm}$

Sek. Ofl.: -

Überzug flächig, Farbe innen: 10R5/8, Farbe außen: 10R5/8, matt, kompakt

Dat.: 5. Jh. n. Chr.

\section{Kat. 81}

Abb. 19

Gattung: LRC; Form: Teller Hayes 4

Inv. 2005/67/1, FO: NT 05, SE 34

Erh.: 1 Bfrgt.

Ton: hart, fein, feinporös; weiße Einschlüsse, fein, häufig

Farbe Kern: 10R5/6, Farbe Rand: 10R5/6

Maße: BDm $10 \mathrm{~cm}$

Überzug flächig, Farbe innen: 10R5/8, Farbe außen: 10R5/8, matt glänzend, kompakt

Dat.: Mitte 5. Jh. n. Chr. (?)

\section{Kat. 82}

Abb. 19

Gattung: ERSW; Form: Teller

Inv. 2005/57/3, FO: NT 05, SE 34

Erh.: 1 Bfrgt.

Ton: mittelhart, fein, feinporös; weiße Einschlüsse, fein, vereinzelt

Farbe Kern: 5YR6/4, Farbe Rand: 5YR6/4

Maße: BDm $12 \mathrm{~cm}$

Sek. Ofl.: -

Überzug flächig, Farbe innen: 7.5YR4/3, Farbe außen: 7.5YR4/3, matt, abgerieben

Dat:: 6. Jh. n. Chr.

\section{Kat. 83}

Abb. 19

Gattung: Amphore; Form: LRA 2

Inv. 2005/57/8, FO: NT 05, SE 34

Bemerkungen: LRA 2

Erh.: 1 Henkel

Ton: hart, mittelfein, mittelporös; Glimmer, fein, vereinzelt; weiße Einschlüsse, fein, viel; weiße Einschlüsse, mittel, mittel; weiße Einschlüsse, groß, vereinzelt

Farbe Kern: 5YR6/6, Farbe Rand: 5YR6/6

Maße: erh. H 6 cm; erh. L 9 cm

Sek. Ofl.: -

Farbe innen: 7.5YR8/4, Farbe außen: 7.5YR8/4

Dat.: Ende 4.-7. Jh. n. Chr.

\section{Kat. 84}

Abb. 19. 25

Gattung: Lampe

Inv. 2005/67/2, FO: NT 05, SE 34

Bemerkungen: rot überfangene Lampe, klein, mit vollem Griff und rotem Überzug

Erh.: zur Hälfte

Ton: hart, fein, feinporös; Glimmer, fein, viel
Farbe Kern: 2.5YR5/6, Farbe Rand: 2.5YR5/6

Maße: BDm 2,4 cm

Sek. Ofl.: -

Überzug außen, Farbe innen: 2.5YR5/6, Farbe außen: 10R5/8

Dat.: spätantik

Kat. 85

Abb. 19

Form: Becher

Inv. NT05/57/G2

Bemerkungen: mit leicht eingebogenem, leicht verdicktem Rand; feine Bläschen, wenig

Erh.: 1 Rfrgt.

Maße: RDm 6,4 cm

Farbe: Y10/M00/C10

Dat.: spätantik

Kat. 86

Abb. 19

Form: Kelchglas

Inv. NT05/57/G1

Bemerkungen: Röhrchenstandring, sehr feine Bläschen

Erh.: 1 Bfrgt.

Maße: BDm 5,4 cm

Farbe: S10/C00/Y60

Dat.: spätantik

Kat. 87

Abb. 20

Gattung: Amphore; Form: Peacock - Williams 45

Inv. 2005/18/2, FO: NT 05, SE 11

Bemerkungen: One Handle Jar

Erh.: 1 Rfrgt.

Ton: hart, fein, feinporös; Glimmer, fein, viel; weiße Einschlüsse, fein, vereinzelt

Farbe Kern: 10R5/8, Farbe Rand: 2.5 YR5/6

Maße: RDm $3 \mathrm{~cm}$

Sek. Ofl.: -

Farbe innen: 10R6/6, Farbe außen: 10R5/6

Dat.: 1. Jh. v. Chr. - 4. Jh. n. Chr.

Kat. 88

Abb. 20

Gattung: Küchenware; Form: Kasserolle

Inv. 2005/26/2, FO: NT 05, SE 11

Bemerkungen: mit innen abgestuftem Rand

Erh.: 1 Rfrgt.

Ton: hart, fein, fein bis mittelporös; Glimmer, fein, vereinzelt; weiße Einschlüsse, fein, vereinzelt

Farbe Kern: 5YR3/3, Farbe Rand: 5YR4/6

Maße: RDm $22 \mathrm{~cm}$

Dat.: 4./5. Jh. n. Chr.

Kat. 89

Abb. 20

Gattung: Lampe

Inv. 2005/79/3, FO: NT 05, SE 11

Bemerkungen: Reste eines mattrötlichen Überzugs und planta pedis-Stempels

Erh.: 1 Bfrgt.

Ton: hart, fein, feinporös; Glimmer, fein, vereinzelt 
Farbe Kern: 2.5YR6/8, Farbe Rand: 2.5YR6/8

Maße: erh. H 0,6 cm; erh. L 2,9 cm; erh. B 2,2 cm

Farbe innen: 2.5YR6/6, Farbe außen: 2.5YR6/6

Sek. Ofl.: teilweise versintert

planta pedis

Stempelverzierung, Überzug außen, Farbe innen: 2.5YR6/6,

Farbe außen: 10R5/4, metallisch glänzend, kompakt

Dat.: spätantik

\section{Kat. 90}

Abb. 20

Gattung: Lampe

Inv. 2005/26/1, FO: NT 05, SE 11

Bemerkungen: mit Bodenrosette, eingefasst von Wulst, davon ausgehend 2 Rippen

Erh.: 1 Bfrgt.

Ton: mittelhart, fein, feinporös; Glimmer, fein, vereinzelt

Farbe Kern: 2.5YR6/6, Farbe Rand: 2.5YR6/6

Maße: erh. H 0,9 cm; erh. L $4 \mathrm{~cm}$

Überzug, außen, Farbe innen: 5YR6/6, Farbe außen: 10R5/6, matt, teilweise abgerieben

Datierung: -

\section{Kat. 91}

Abb. 20

Gattung: ARS; Form: Teller Hayes 108

Inv. 2005/12/3, FO: NT 05, SE 10

Bemerkungen: ARS 2 (relativ grobes Fabric)

Erh.: 1 Rfrgt.

Ton: hart, mittelfein, mittelporös; weiße Einschlüsse, fein, mittel

Farbe Kern: 10R5/6, Farbe Rand: 10R5/6

Maße: RDm $22 \mathrm{~cm}$

Überzug flächig, Farbe innen: 10R5/8, Farbe außen: 10R5/8, matt glänzend, kompakt

Dat.: frühes 7. Jh. n. Chr.

\section{Kat. 92}

Abb. 20

Gattung: ARS; Form: Hayes 105

Inv. 2005/12/4, FO: NT 05, SE 10

Bemerkungen: kleines Rfrgt., verbrannt

Erh.: 1 Rfrgt.

Ton: hart, fein, feinporös; Glimmer, fein, vereinzelt; weiße Einschlüsse, fein, vereinzelt; graue Einschlüsse, fein, vereinzelt; rote Einschlüsse, fein, vereinzelt

Farbe Kern: 7.5YR3/2, Farbe Rand: 7.5YR3/2

Maße: RDm $18 \mathrm{~cm}$

Sek. Ofl.: verbrannt

Überzug flächig, Farbe innen: 2.5YR5/4; Farbe außen:

2.5YR5/4, matt glänzend, kompakt

Dat.: spätes 6.-7. Jh. n. Chr

\section{Kat. 93}

Abb. 20

Gattung: ARS; Form: Teller Hayes 50B

Inv. 2005/27/1, FO: NT 05, SE 10

Erh.: 1 Rfrgt.

Ton: hart, sehr fein, sehr feinporös

Farbe Kern: 10R5/8, Farbe Rand: 10R5/8

Maße: RDm $20 \mathrm{~cm}$
Überzug flächig, Farbe innen: 10R5/8, Farbe außen: 10R5/8, matt glänzend, kompakt

Dat.: 4. Jh. n. Chr.

Kat. 94

Abb. 20

Gattung: LRC; Form: Teller Hayes 3/10

Inv. 2005/12/2, FO: NT 05, SE 10

Bemerkungen: am Rand außen leichte Kerbreihe sichtbar

Erh.: 1 Rfrgt.

Ton: hart, fein, feinporös; Glimmer, fein, vereinzelt; weiße

Einschlüsse, fein, vereinzelt

Farbe Kern: 10R5/6, Farbe Rand: 10R5/6

Maße: RDm $25 \mathrm{~cm}$

Dekor: Kerben, Überzug flächig, Farbe innen: 10R5/8, Farbe außen: 10R5/8, matt, teilweise abgerieben

Dat.: 6. Jh. n. Chr.

Kat. 95

Abb. 20

Gattung: LRC; Form: Teller Hayes 3C

Inv. 2005/12/1, FO: NT 05, SE 10

Erh.: 1 Rfrgt.

Ton: hart, fein, feinporös; Glimmer, fein, vereinzelt; weiße Einschlüsse, sehr fein, viel

Farbe Kern: 10R5/6, Farbe Rand: 10R5/6

Maße: RDm $19 \mathrm{~cm}$

Überzug flächig, Farbe innen: 10R4/6, Farbe außen: 10R4/6, matt glänzend, kompakt

Dat.: spätes 5. Jh. n. Chr.

Kat. 96

Abb. 20

Gattung: Amphore; Form: Ephesos 56

Inv. NT05/21/2, FO: NT 05, SE 10

Erh.: 1 Bfrgt.

Ton: sehr hart, fein, feinporös; Glimmer, fein, viel

Farbe Kern: 10YR6/4, Farbe Rand: 10YR6/4

Maße: BDm ca. $1,5 \mathrm{~cm}$

Farbe innen: 10R4/6, Farbe außen: 10R4/6

Dat.: 6. Jh. n. Chr.

Kat. 97

Abb. 20

Gattung: Amphore; Form: LRA 3

Inv. 2005/12/21, FO: NT 05, SE 10

Bemerkungen: spätes Rfrgt. (ab 4. Jh.)

Erh.: 1 Rfrgt.

Ton: hart, fein, feinporös; Glimmer, fein bis mittel, viel; gelbliche Einschlüsse, mittel, selten

Farbe Kern: 2.5YR6/6, Farbe Rand: 2.5YR6/6

Maße: RDm 4 cm

Dat.: 4.-6. Jh. n. Chr.

Kat. 98

Abb. 20

Gattung: Amphore; Form: LRA 3

Inv. 2005/27/10, FO: NT 05, SE 10

Erh.: 1 Rfrgt.

Ton: hart, fein, feinporös; Glimmer, fein, viel

Farbe Kern: 5YR3/4, Farbe Rand: 5YR3/4 
Maße: RDm 4,4 cm

Dat.: 4.-6. Jh. n. Chr.

\section{Kat. 99}

Gattung: Lampe; Form: Doppelschnauzenlampe

Inv. 2005/25/1, FO: NT 05, SE 10

Bemerkungen: Reste von 2 Fülllöchern, kantig

Erh.: 1 Henkel

Ton: hart, sehr fein, feinporös; Glimmer, fein, mittel

Farbe Kern: 5YR6/6, Farbe Rand: 5YR6/6

Maße: erh. H 3,5 cm; erh. L 5,8 cm

Sek. Ofl.: -

Farbe innen: 7.5YR7/4, Farbe außen: 7.5YR7/4

Dat.: spätantik (5. Jh. n. Chr.)

\section{Kat. 100}

Abb. 21. 25

Gattung: Lampe

Inv. 2005/78/12, FO: NT 05, SE 10

Bemerkungen: mit Fischgrätmuster, innen mattroter Überzug Erh.: 1 Schnauze

Ton: hart, fein, feinporös; weiße Einschlüsse, sehr fein, vereinzelt

Farbe Kern: 2.5YR5/6, Farbe Rand: 2.5YR5/6

Maße: erh. H ca. $1 \mathrm{~cm}$; erh. L $5 \mathrm{~cm}$

Sek. Ofl.: -

Überzug flächig, Farbe innen: 10R4/4, Farbe außen: 2.5YR6/6, matt, abgerieben

Dat.: spätantik (5. Jh. n. Chr.)

\section{Kat. 101}

Abb. 21

\section{Gattung: Lampe}

Inv. 2005/78/8, FO: NT 05, SE 10

Bemerkungen: rot überfangen, mit Rosette am Boden

Erh.: 1 Bfrgt.

Ton: hart, fein, feinporös; dunkelgraue Einschlüsse, fein, vereinzelt; weiße Einschlüsse, fein, vereinzelt

Farbe Kern: 2.5YR5/8, Farbe Rand: 2.5YR5/8

Maße: BDm $4 \mathrm{~cm}$

Sek. Ofl.: -

Überzug außen, Farbe innen: 10R6/8, Farbe außen: 10R5/8, matt, abgerieben

Dat.: spätantik (?)

\section{Kat. 102}

Abb. 21

Gattung: Glas; Form: Lampe

Inv. NT05/12/G1

Bemerkungen: Glaslampe mit Stielfuß, vereinzelt feine Luftbläschen

Erh.: 1 Bfrgt.

Farbe: Y10/M00/C10

Maße: BDm ca. $0,6 \mathrm{~cm}$

Dat.: ab 4./5. Jh. n. Chr.

\section{Kat. 103}

Abb. 21
Erh.: 1 Rfrgt.

Ton: hart, fein, feinporös; Glimmer, fein, vereinzelt; weiße Einschlüsse, fein, vereinzelt

Farbe Kern: 2.5YR6/6, Farbe Rand: 2.5YR6/8

Maße: RDm $18 \mathrm{~cm}$

Überzug flächig, Farbe innen: 10R5/8, Farbe außen: 10R5/8, matt, abgerieben

Dat.: 6. Jh. n. Chr.

Kat. 104

Abb. 21

Gattung: LRC; Form: Teller Hayes 3

Inv. 2005/96/1, FO: NT 05, SE 7

Erh.: 1 Rfrgt.

Ton: hart, fein, feinporös; Glimmer, fein, vereinzelt

Farbe Kern: 10R5/6, Farbe Rand: 10R6/8

Maße: RDm $20 \mathrm{~cm}$

Überzug flächig, Farbe innen: 10R5/8, Farbe außen: 10R5/8, matt, kompakt

Dat.: 5. Jh. n. Chr.

\section{Kat. 105}

Abb. 21

Gattung: LRC; Form: Teller Hayes 3

Inv. 2005/96/2, FO: NT 05, SE 7

Erh.: 1 Rfrgt.

Ton: hart, fein, feinporös; Glimmer, fein, vereinzelt; weiße Einschlüsse, fein, vereinzelt

Farbe Kern: 5YR6/6, Farbe Rand: 5YR6/6

Maße: RDm $22 \mathrm{~cm}$

Überzug, flächig, Farbe innen: 10R6/8, Farbe außen: 10R6/8, matt, teilweise abgerieben

Dat.: 5. Jh. n. Chr.

\section{Kat. 106}

Abb. 21

Gattung: LRC; Form: Teller Hayes 3

Inv. 2005/11/1, FO: NT 05, SE 9

Erh.: 1 Bfrgt.

Ton: hart, fein, feinporös; Glimmer, fein, vereinzelt; weiße Einschlüsse, fein, vereinzelt

Farbe Kern: 10R6/8, Farbe Rand: 10R6/8

Maße: BDm 6,2 cm

Sek. Ofl.: teilweise versintert

Überzug, flächig, Farbe innen: 10R6/8, Farbe außen: 10R6/8, matt, kompakt

Dat.: 5./6. Jh. n. Chr.

Kat. 107

Abb. 21

Gattung: LRC; Form: Teller Hayes 3

Inv. 2005/96/3, FO: NT 05, SE 7

Erh.: 1 Bfrgt.

Ton: hart, fein, feinporös; Glimmer, fein, vereinzelt; weiße Einschlüsse, fein, vereinzelt

Farbe Kern: 2.5YR6/8, Farbe Rand: 2.5YR6/8

Maße: BDm $10 \mathrm{~cm}$

Überzug flächig, Farbe innen: 10R6/8, Farbe außen: 10R6/8, matt, kompakt

Dat.: 5./6. Jh. n. Chr. 


\section{Kat. 108}

Gattung: LRC; Form: Schälchen Hayes 2

Inv. 2005/9/1, FO: NT 05, SE 7

Bemerkungen: kleines Schälchen, vgl. Ladstätter - Sauer 2005, 14; stark versintert

Erh.: 1 Rfrgt.

Ton: hart, fein, feinporös; Glimmer, fein, vereinzelt; weiße Einschlüsse, fein, vereinzelt

Farbe Kern: 2.5YR6/8, Farbe Rand: 10R6/8

Maße: RDm $10 \mathrm{~cm}$

Sek. Ofl.: versintert

Überzug flächig, Farbe innen: 2.5YR4/6, Farbe außen: 2.5YR4/6, matt, kompakt

Dat.: spätes 4./1. Hälfte 5. Jh. n. Chr.

\section{Kat. 109}

Abb. 21

Gattung: Gebrauchskeramik; Form: frühbyzantinische Ampulle

Inv. 2005/96/5, FO: NT 05, SE 7

Bemerkungen: mit flachem Boden, kein Stempel, Überzugrest außen abgeronnen

Erh.: 1 Bfrgt.

Ton: hart, fein, feinporös; Glimmer, fein, vereinzelt; weiße Einschlüsse, fein, vereinzelt

Farbe Kern: 5YR7/4, Farbe Rand: 5YR7/4

Maße: BDm 2,6 cm

Überzug, partiell, Farbe innen: 5YR6/4, Farbe außen: 5YR7/4, matt, teilweise abgerieben

Dat.: 5./6. Jh. n. Chr.

\section{Kat. 110}

Abb. 21

Gattung: Amphore

Inv. 2005/9/7, FO: NT 05, SE 7

Bemerkungen: mit verdicktem, außen abgesetztem Rand, Ofl. glimmrig, Rest des Henkelansatzes; wohl lokal (Ofl. glimmrig wie LRA 3, Fabric mit Glimmer)

Erh.: 1 Rfrgt.

Ton: hart, fein, feinporös; Glimmer, fein, vereinzelt

Farbe Kern: 5YR6/4, Farbe Rand: 5YR4/3

Maße: RDm 4,3 cm

Sek. Ofl.: -

Farbe innen: 5YR6/6, Farbe außen: 7.5YR7/4

Dat.: -

\section{Kat. 111}

Gattung: Amphore; Form: LRA 3

Inv. 2005/9/15, FO: NT 05, SE 7

Erh.: 1 Rfrgt. und 2 Hfrgte.

Ton: mittelhart, fein, feinporös; Glimmer, fein, viel; weiße Einschlüsse, fein, vereinzelt

Farbe Kern: 2.5YR4/8, Farbe Rand: 2.5YR4/8

Maße: RDm 3,7 cm

Farbe innen: 10R5/4, Farbe außen: 10R5/4

Dat.: 4.-6. Jh. n. Chr.

\section{Kat. 112}

Gattung: Glas; Form: Becher Inv. 2005/11/14, FO: NT 05, SE 9
Bemerkungen: mit Schliffrillen am Rand außen; blasig, schlierig

Erh.: 1 Rfrgt.

Farbe: S00/C00/Y20

Maße: RDm $8 \mathrm{~cm}$

Sek. Ofl.: Patina

Dat.: kaiserzeitlich - spätantik

\section{Kat. 113}

Abb. 22

Gattung: ARS; Form: Teller Hayes 57

Inv. 2005/97/1, FO: NT 05, SE 30

Erh.: 1 Rfrgt.

Ton: hart, fein, feinporös, mit freiem Auge keine Einschlüsse erkennbar

Farbe Kern: 10R6/8, Farbe Rand: 10R6/8

Maße: RDm $23 \mathrm{~cm}$

Überzug flächig, Farbe innen: 10R5/8, Farbe außen: 10R6/8, matt glänzend, kompakt

Dat.: $325-400$ n. Chr.

Kat. 114

Abb. 22

Gattung: ERSW; Form: Schale Hayes 91/92

Inv. 2005/51/1, FO: NT 05, SE 30

Bemerkungen: Steilrandschale, Imitation ARS Hayes 91/92; Rand weiß (2.5YR8/1) gebrannt; Ofl. glimmrig; vgl. Ladstätter - Sauer 2005, Taf. 14 EPH-HH-TS 02

Erh.: 1 Ganzform

Ton: hart, fein, feinporös; Glimmer, sehr fein, vereinzelt; weiße Einschlüsse, sehr fein, vereinzelt

Farbe Kern: 2.5YR6/6, Farbe Rand: 2.5YR6/6

Maße: RDm $12 \mathrm{~cm}, \mathrm{BDm}$ 4,6 cm

Sek. Ofl.: versintert

Überzug flächig, Farbe innen: 10R6/6, Farbe außen: 2.5YR7/4, matt, abgerieben

Dat.: 6. Jh. n. Chr.

Kat. 115

Abb. 22

Gattung: ERSW; Form: Teller Hayes 1B

Inv. 2005/51/2, FO: NT 05, SE 30

Erh.: 1 Rfrgt.

Ton: mittelhart, fein, feinporös; Glimmer, fein, vereinzelt

Farbe Kern: 2.5YR6/6, Farbe Rand: 2.5YR6/6

Maße: RDm $24 \mathrm{~cm}$

Sek. Ofl.: -

Überzug flächig, Farbe innen: 2.5YR7/6, Farbe außen: 10R5/8, matt, abgerieben

Dat.: 6. Jh. n. Chr.

Kat. 116

Abb. 22. 25

Inv. NT05/53/x; Form: frühbyzantinische Ampulle

Erh.: 1 Rfrgt.

Ton: hart, fein, feinporös; weiße Einschlüsse, sehr fein, vereinzelt

Farbe Kern: 10R5/1, Farbe Rand: 10R6/6-5/6

Maße: RDm $4 \mathrm{~cm}$

Überzug flächig, Farbe innen: 10R6/6, Farbe außen: 10R6/6

Dat.: 6. Jh. n. Chr. 


\section{Kat. 117}

Abb. 22

Gattung: Lampe

Inv. 2005/51/3 (Museum KF NT 2005/29), FO: NT 05, SE

30

Bemerkungen: rot überfangene Lampe, Brandspuren an der Schnauze

Erh.: 1 Ganzform

Ton: nicht zu beurteilen, da Überzug kompakt

Maße: H 3,5 cm; L 7,7 cm; B 5,7 cm

Überzug flächig, Farbe außen: 10YR5/6, matt, kompakt

Dat.: spätantik

\section{Kat. 118}

Gattung: LRC; Form: Teller Hayes 3

Abb. 22

Inv. 2005/6/3, FO: NT 05, SE 4

Erh.: 1 Rfrgt.

Ton: hart, fein, feinporös; Glimmereinschlüsse, fein, vereinzelt; weiße Einschlüsse, fein, vereinzelt

Farbe Kern: 2.5YR6/6, Farbe Rand: 2.5YR6/6

Maße: RDm $23 \mathrm{~cm}$

Überzug flächig, Farbe innen: 10R5/8, Farbe außen: 10R5/6, matt, kompakt

Dat.: 5./6. Jh. n. Chr.

\section{Kat. 119}

Abb. 22

Gattung: LRC; Form: Teller Hayes 3

Inv. 2005/94/3, FO: NT 05, SE 4

Bemerkungen: klein, Rand außen leicht gekehlt

Erh.: 1 Rfrgt.

Ton: mittelhart, fein, feinporös; Glimmer, fein, vereinzelt; weiße Einschlüsse, fein, vereinzelt

Farbe Kern: 2.5YR6/8, Farbe Rand: 2.5YR6/8

Maße: RDm 14,8 cm

Überzug flächig, Farbe innen: 2.5YR6/8, Farbe außen: 10R6/8, matt, kompakt

Dat.: 5. Jh. n. Chr.

\section{Kat. 120}

Gattung: LRC; Form: Teller Hayes 3H

Inv. 2005/94/4, FO: NT 05, SE 4

Erh.: 1 Rfrgt.

Ton: hart, fein, feinporös; Glimmer, sehr fein, vereinzelt; weiße Einschlüsse, fein, vereinzelt

Farbe Kern: 2.5YR6/8, Farbe Rand: 2.5YR6/8

Maße: RDm $28 \mathrm{~cm}$

Überzug, flächig, Farbe innen: 2.5YR6/8, Farbe außen: 10R6/8, matt, teilweise abgerieben

Dat.: 6. Jh. n. Chr.

Kat. 121

Abb. 23

Gattung: LRC; Form: Teller Hayes 3

Inv. 2005/94/2, FO: NT 05, SE 4

Erh.: 1 Bfrgt.

Ton: hart, fein, feinporös; Glimmer, sehr fein, vereinzelt; weiße Einschlüsse, fein, vereinzelt

Farbe Kern: 2.5YR5/6, Farbe Rand: 2.5YR5/6

Maße: BDm $8 \mathrm{~cm}$
Überzug flächig, Farbe innen: 10R5/8, Farbe außen: 10R5/8, matt, kompakt

Dat:: 5./6. Jh. n. Chr.

Kat. 122

Abb. 23

Gattung: ERSW; Form: Teller Hayes 8

Inv. 2005/16/2, FO: NT 05, SE 4

Erh.: 1 Rfrgt.

Ton: hart, fein, feinporös; Glimmer, fein, mittel

Farbe Kern: 2.5YR6/8, Farbe Rand: 2.5YR6/8

Maße: RDm 16,4 cm

Sek. Ofl.: versintert

Überzug Rand innen und außen, Farbe innen: 2.5YR7/6-6/6, Farbe außen: 2.5YR6/4-6/6 matt, teilweise abgerieben

Dat.: 6./7. Jh. n. Chr.

\section{Kat. 123}

Abb. 23

Gattung: ERSW; Form: Teller Hayes 10

Inv. 2005/16/3, FO: NT 05, SE 4

Bemerkungen: Rand heller gebrannt, grob

Erh.: 1 Rfrgt.

Ton: hart, fein, feinporös; Glimmer, fein, mittel

Farbe Kern: 5YR5/8, Farbe Rand: 5YR5/8

Maße: RDm $29 \mathrm{~cm}$

Überzug flächig, Farbe innen: 10R5/4-5/6; Farbe außen: 10R5/4-5/6, matt, teilweise abgerieben Dat.: 6./7. Jh. n. Chr.

Kat. 124

Abb. 23

Gattung: Amphore; Form: Ephesos 56

Inv. 2005/94/17, FO: NT 05, SE 4

Erh.: 1 Rfrgt.

Ton: hart, fein, feinporös; Glimmer, fein, vereinzelt; weiße Einschlüsse, fein, vereinzelt; schwarze Einschlüsse, fein, vereinzelt

Farbe Kern: 2.5YR6/8, Farbe Rand: 2.5YR6/8

Maße: RDm $5 \mathrm{~cm}$

Sek. Ofl.: flächig versintert

Farbe innen: 10R6/8, Farbe außen: 10R6/8

Dat.: 6. Jh. n. Chr.

\section{Kat. 125}

Abb. 23

Gattung: Amphore; Form: Ephesos 56

Inv. 2005/94/16, FO: NT 05, SE 4

Bemerkungen: abgebrochen; Ofl. glimmrig

Erh.: 1 Bfrgt.

Ton: hart, fein, feinporös; Glimmer, fein, vereinzelt

Farbe Kern: 2.5YR5/2, Farbe Rand: 5YR4/6

Maße: BDm 2,8 cm

Farbe innen: 2.5YR6/4, Farbe außen: 2.5YR6/6

Dat.: 6. Jh. n. Chr.

Kat. 126

Abb. 23

Gattung: LRC; Form: Teller Hayes 3/10

Inv. 2005/74/3, FO: NT 05, SE 1

Erh.: 1 Rfrgt. 
Ton: sehr hart, fein, feinporös; Glimmer, fein, vereinzelt; helle Einschlüsse, fein, vereinzelt; dunkle Einschlüsse, fein, vereinzelt

Farbe Kern: 2.5YR5/6, Farbe Rand: 2.5YR5/6

Maße: RDm $26 \mathrm{~cm}$

Überzug, flächig, Farbe innen: 10R5/6, Farbe außen: 10R5/8, matt, kompakt

Dat.: 6. Jh. n. Chr.

\section{Kat. 127}

Gattung: Amphore; Form: LRA 3

Inv. 2005/6/17, FO: NT 05, SE 4

Bemerkungen: Fabric ähnlich LRA 3, mit Graffito (wohl ein Brief oder eine Mitteilung):

meleie (eta) is

$$
\begin{aligned}
& \text { poneron (Eta, Omega) } \\
& \text {-eios } \\
& \text {-stra(?) } \\
& \text {-po- }^{184}
\end{aligned}
$$

Erh.: 1 Wfrgt.

Ton: mittelhart, fein, feinporös; Glimmer, fein, mittel; weiße Einschlüsse, fein, vereinzelt

Farbe Kern: 10YR4/4, Farbe Rand: 2.5YR4/8

Maße: erh. H 5 cm; erh. B $6 \mathrm{~cm}$

Sek. Ofl.: -

Farbe innen: 10R4/6, Farbe außen: 2.5YR5/4

Dat.: spätantik

\section{Kat. 128}

Abb. 23

Gattung: LRC; Form: Teller Hayes 3F oder 3/10

Inv. 2005/2/5, FO: NT 05, SE 1 (mörtelige Erde, flächig im Schnitt)

Erh.: 1 Rfrgt.

Ton: sehr hart, fein, mittelporös; Glimmer, sehr fein, vereinzelt; helle Einschlüsse, fein, mittel

Farbe Kern: 2.5YR5/6, Farbe Rand: 2.5YR5/6

Maße: RDm $29 \mathrm{~cm}$

Überzug, flächig, Farbe innen: 10R5/8, Farbe außen: 10R5/8, matt, kompakt

Dat.: 6. Jh. n. Chr

\section{Kat. 129}

Abb. 23

Gattung: LRC; Form: Teller Hayes 3/10

Inv. 2005/74/2, FO: NT 05, SE 1

Bemerkungen: außen und über Rand: fleckig (10R4/6-

2.5YR6/6), innen: matt, teilweise abgerieben

Erh.: 1 Rfrgt.

Ton: sehr hart, fein, feinporös; Glimmer, fein, vereinzelt; helle Einschlüsse, sehr fein, mittel; dunkle Einschlüsse, sehr fein, mittel

Farbe Kern: 2.5YR6/8, Farbe Rand: 2.5YR6/8

Maße: RDm $25 \mathrm{~cm}$

Überzug, flächig, Farbe innen: 10R5/8, Farbe außen: 10R4/6, matt, kompakt

Dat.: 6. Jh. n. Chr

\section{Kat. 130}

Gattung: LRC; Form: Hayes 6

Inv. 2005/90/1, FO: NT 05, SE 1

Erh.: 1 Rfrgt.

Ton: hart, fein, feinporös; weiße Einschlüsse, fein, viel

Farbe Kern: 2.5YR5/8, Farbe Rand: 2.5YR5/8

Maße: RDm $26 \mathrm{~cm}$

Überzug: flächig; Farbe innen: 10R4/8, Farbe außen: 10R4/8, matt glänzend, kompakt

Dat.: 6. Jh. n. Chr.

\section{Kat. 131}

Gattung: LRC; Form: Teller Hayes 3

Abb. 23

Inv. 2005/74/4, FO: NT 05, SE 1

Bemerkungen: kleiner Rest eines nicht näher identifizierbaren Stempels in der Bodenmitte

Erh.: 1 Bfrgt.

Ton: hart, fein, feinporös; Glimmer, fein, vereinzelt; helle Einschlüsse, fein, viel

Farbe Kern: 2.5YR5/4, Farbe Rand: 2.5YR5/4

Maße: BDm 13,4 cm

Sek. Ofl.: -

Überzug, flächig, Farbe innen: 10R5/6, Farbe außen: 10R5/6, matt, kompakt

Dat.: 6. Jh. n. Chr.

Kat. 132

Abb. 23

Gattung: LRC; Form: Teller

Inv. 2005/2/13, FO: NT 05, SE 1 (mörtelige Erde, flächig im Schnitt)

Bemerkungen: Stempel Hayes A

Erh.: 1 Bfrgt.

Ton: hart, fein, feinporös; Glimmer, sehr fein, vereinzelt; helle Einschlüsse, fein, vereinzelt; dunkle Einschlüsse, sehr fein, vereinzelt

Farbe Kern: 2.5YR6/8, Farbe Rand: 2.5YR6/8

Maße: erh. H ca. 0,4 cm; erh. L/B 5,4 cm $\times 2,8 \mathrm{~cm}$

Stempelverzierung, Überzug flächig, Farbe innen: 2.5YR6/8, Farbe außen: 2.5YR6/8, matt, abgerieben

Dat.: 6. Jh. n. Chr.

Kat. 133

Abb. 23

Gattung: ERSW; Form: Teller Hayes 3

Inv. 2005/74/1, FO: NT 05, SE 1

Erh.: 1 Rfrgt.

Ton: hart, fein, feinporös; Glimmer, sehr fein, viel; dunkle Einschlüsse, sehr fein, vereinzelt

Farbe Kern: 5YR5/6, Farbe Rand: 5YR5/6

Maße: RDm 18,8 cm

Überzug innen und Rand außen, Farbe innen: 2.5YR5/6, Farbe außen: 2.5 YR5 5 , matt, teilweise abgerieben

Dat.: 6. Jh. n. Chr.

Abb. 23

Gattung: ERSW; Form: Hayes 8

Inv. 2005/13/7, FO: NT 05, SE 1

\footnotetext{
${ }^{184}$ Lesung H. Taeuber.
} 
Bemerkungen: am Rand außen Kerbdekor

Erh.: 1 Rfrgt.

Ton: hart, sehr fein, feinporös; Glimmer, fein, vereinzelt

Farbe Kern: 5YR6/4, Farbe Rand: 5YR6/4

Maße: RDm $12 \mathrm{~cm}$

Kerben, Überzug innen und Rand außen, Farbe innen: Farbe außen: 5YR7/4-4/2, Farbe außen: 5YR6/4, matt, kompakt

Dat.: 6. Jh. n. Chr.

\section{Kat. 135}

Gattung: ERSW; Form: Hayes 3

Inv. 2005/90/3, FO: NT 05, SE 1

Bemerkungen: grob, rau, kaum Überzugreste

Erh.: 1 Rfrgt.

Ton: hart, fein, feinporös; Glimmer, fein, mittel; weiße Einschlüsse, fein, mittel; Quarz, fein, vereinzelt

Farbe Kern: 5YR5/3, Farbe Rand: 5YR5/3

Maße: RDm $20 \mathrm{~cm}$

Dat.: 6. Jh. n. Chr.

\section{Kat. 136}

Abb. 24

Gattung: ARS; Form: Teller Hayes 108

Inv. 2005/2/4, FO: NT 05, SE 1 (mörtelige Erde, flächig im Schnitt)

Erh.: 1 Rfrgt.

Ton: hart, mittelfein, mittelporös; helle Einschlüsse, fein, vereinzelt; dunkle Einschlüsse, fein, mittel; Glimmer, sehr fein, viel

Farbe Kern: 10R5/6, Farbe Rand: 2.5YR5/8

Maße: RDm $28 \mathrm{~cm}$

Überzug flächig, Farbe innen: 10R5/8, Farbe außen: 10R5/8, matt glänzend, kompakt

Dat.: Anfang 7. Jh. n. Chr.

Kat. 137

Abb. 24

Gattung: ARS; Form: Teller Hayes 104C

Inv. 2005/2/9, FO: NT 05, SE 1 (mörtelige Erde, flächig im Schnitt)

Bemerkungen: außen über dem Standring Rillen

Erh.: 1 Bfrgt.

Ton: hart, fein, feinporös; Glimmer, sehr fein, vereinzelt; helle Einschlüsse, fein, vereinzelt; graue Einschlüsse, fein, vereinzelt

Farbe Kern: 2.5YR6/8, Farbe Rand: 2.5YR6/8

Maße: BDm $11 \mathrm{~cm}$

Überzug flächig, Farbe innen: 2.5YR5/8, Farbe außen:

2.5YR5/8, matt, kompakt

Dat.: Mitte 6./Anfang 7. Jh. n. Chr.

Kat. 138

Gattung: ERSW; Form: Schale Hayes 9B

Inv. 2005/13/2, FO: NT 05, SE 1

Bemerkungen: Rand außen: 10YR7/3

Erh.: 1 Rfrgt.
Ton: hart, sehr fein, feinporös; gelblich weiße Einschlüsse, fein, vereinzelt

Farbe Kern: 2.5YR5/6, Farbe Rand: 2.5YR5/6

Maße: RDm $14 \mathrm{~cm}$

Überzug flächig, Farbe innen: 10R5/6, Farbe außen: 10R6/6, matt, teilweise abgerieben

Dat.: 6. Jh. n. Chr

Kat. 139

Abb. 24

Gattung: Gebrauchskeramik; Form: frühbyzantinische Ampulle Inv. 2005/74/5, FO: NT 05, SE 1

Erh.: 1 Rfrgt.

Ton: hart, fein, feinporös; Glimmer, fein, vereinzelt; helle Einschlüsse, sehr fein, vereinzelt

Farbe Kern: 2.5YR4/4, Farbe Rand: 2.5 YR5/6

Maße: RDm $3 \mathrm{~cm}$

Überzug partiell, Farbe innen: 7.5YR4/6, Farbe außen: GLEY1

$3 / \mathrm{N}$, matt, teilweise abgerieben

Dat.: 5./6. Jh. n. Chr.

Kat. 140

Abb. 24

Gattung: Amphore; Form: LRA 3

Inv. 2005/13/6, FO: NT 05, SE 1

Bemerkungen: geschlossener Knauf, unten abgebrochen

Erh.: 1 Knauf

Ton: hart, fein, feinporös; Glimmer, fein, viel

Farbe Kern: 2.5YR4/4, Farbe Rand: 2.5YR4/4

Maße: Dm Knaufabsatz $4 \mathrm{~cm}$

Überzug, flächig, Farbe innen: 2.5YR4/3, Farbe außen: 2.5YR4/3, matt, teilweise abgerieben

Dat.: 5./6. Jh. n. Chr.

\section{Kat. 141}

Abb. 24. 25

Gattung: Amphore; Form: LRA 3

Inv. 2005/2/11, FO: NT 05, SE 1 (mörtelige Erde, flächig im Schnitt)

Bemerkungen: Graffito (wohl Mitteilung):

-os epe(m)pse polo(n)

-s to chron(i?)o (am Schluss Omega) ${ }^{185}$

Erh.: 1 Wfrgt.

Ton: hart, fein, feinporös; Glimmer, sehr fein, vereinzelt; helle Einschlüsse, fein, vereinzelt; dunkle Einschlüsse, fein, vereinzelt

Farbe Kern: 5YR4/6, Farbe Rand: 10YR5/3

Maße: erh. H 8 cm; erh. B ca. $6 \mathrm{~cm}$

Überzug, flächig, Farbe innen: 10YR5/6, Farbe außen: 5YR6/4, matt, teilweise abgerieben Dat: $5 . / 6$. Jh. n. Chr.

Kat. 142

Gattung: LRC; Form: Teller Hayes 10C

Abb. 24 Inv. 2005/3/1, FO: NT 05, SE 2

Erh.: 1 Rfrgt.

\footnotetext{
185 Lesung H. Taeuber.
} 
Ton: hart, fein, feinporös; Glimmer, fein, vereinzelt; weiße Einschlüsse, fein, vereinzelt; schwarze Einschlüsse, fein, vereinzelt

Farbe Kern: 2.5YR6/8, Farbe Rand: 2.5YR6/8

Maße: RDm $24 \mathrm{~cm}$

Sek. Ofl.: versintert

Überzug, flächig, Farbe innen: 10R6/8, Farbe außen: 10R6/8, matt, kompakt

Dat.: 1. Hälfte 7. Jh. n. Chr.

\section{Kat. 143}

Abb. 24

Gattung: ERSW; Form: Teller Hayes 10

Inv. 2005/3/5, FO: NT 05, SE 2

Bemerkungen: Ofl. glimmrig

Erh.: 1 Rfrgt.

Ton: weich, fein, feinporös; Glimmer, fein, viel

Farbe Kern: 5YR6/6, Farbe Rand: 5YR6/6

Maße: RDm $32 \mathrm{~cm}$

Überzug, flächig, Farbe innen: 10R4/8, Farbe außen: 10R4/8, matt, abgerieben

Dat.: 1. Hälfte 7. Jh. n. Chr.

\section{Kat. 144}

Abb. 24

Gattung: LRC; Form: Teller Hayes 3-6

Inv. 2005/86/16, FO: NT 05, SE 2

Bemerkungen: Stempelstil Gruppe III, Pfau

Erh.: 1 Bfrgt.

Ton: hart, fein, feinporös; Glimmer, sehr fein, vereinzelt; weiße Einschlüsse, fein, vereinzelt

Farbe Kern: 2.5YR6/6, Farbe Rand: 2.5YR6/6

Maße: erh. L $6 \mathrm{~cm}$

Stempelverzierung figürlich, Überzug flächig, Farbe innen: 10R4/8, Farbe außen: 10R5/6, matt glänzend, kompakt

Dat.: 6. Jh. n. Chr.

\section{Kat. 145}

Abb. 24

Gattung: ERSW; Form: Teller Hayes 3

Inv. 2005/3/6, FO: NT 05, SE 2

Erh.: 1 Bfrgt.

Ton: hart, fein, fein bis mittelporös; Glimmer, fein, vereinzelt; weiße Einschlüsse, fein, vereinzelt

Farbe Kern: 5YR6/6, Farbe Rand: 5YR6/6

Maße: BDm $13 \mathrm{~cm}$

Überzug flächig, Farbe innen: 10R4/6, Farbe außen: 10R4/6, matt, teilweise abgerieben

Dat.: 6. Jh. n. Chr.

\section{Kat. 146}

Gattung: Amphore; Form: LRA 3

Inv. 2005/87/2, FO: NT 05, SE 2

Bemerkungen: geschlossener Knauf

Erh.: 1 Knauf

Ton: hart, fein, feinporös; Glimmer, fein, viel

Farbe Kern: 2.5 YR6/8, Farbe Rand: 2.5YR6/8

Maße: BDm 2,1 cm

Sek. Ofl.: partiell versintert

Farbe innen: 7.5YR6/4, Farbe außen: GLEY1 5/N

Dat.: 6. Jh. n. Chr.

Abb. 24
Kat. 147

Abb. 24. 25

Gattung: Amphore; Form: Spatheion

Inv. 2005/86/3, FO: NT 05, SE 2

Bemerkungen: kleines Spatheion M. Bonifay Typ 3/Variante C

Erh.: 1 Rfrgt.

Ton: hart, fein, feinporös; Glimmer, fein, vereinzelt; dunkle Einschlüsse, fein, mittel; weiße Einschlüsse, fein, vereinzelt

Farbe Kern: 7.5YR7/4, Farbe Rand: 7.5YR8/4

Maße: RDm $6 \mathrm{~cm}$

Überzug, flächig, Farbe innen: 10YR8/4, Farbe außen: 10YR8/4, matt, kompakt

Dat.: 2. Hälfte 7. Jh. n. Chr

\section{Kat. 148}

Abb. 24. 25

Gattung: Lampe

Inv. 2005/3/16, FO: NT 05, SE 2

Bemerkungen: Schnauze abgebrochen, voller Griff, auf der Schulter 6-reihiger Warzendekor; leichter Rippendekor auch vom Griff zum Boden, außen und partiell innen matt rötlich überzogen

Erh.: fast vollständig

Ton: mittelhart, fein, feinporös; Glimmer, fein, vereinzelt Farbe Kern: 2.5YR6/6, Farbe Rand: 2.5YR6/6

Maße: erh. H 6,4 cm; BDm ca. 2,4 cm

Überzug außen, Farbe innen: 2.5YR6/6, Farbe außen: 10R5/8, matt, teilweise abgerieben

Dat.: 6. Jh. n. Chr 

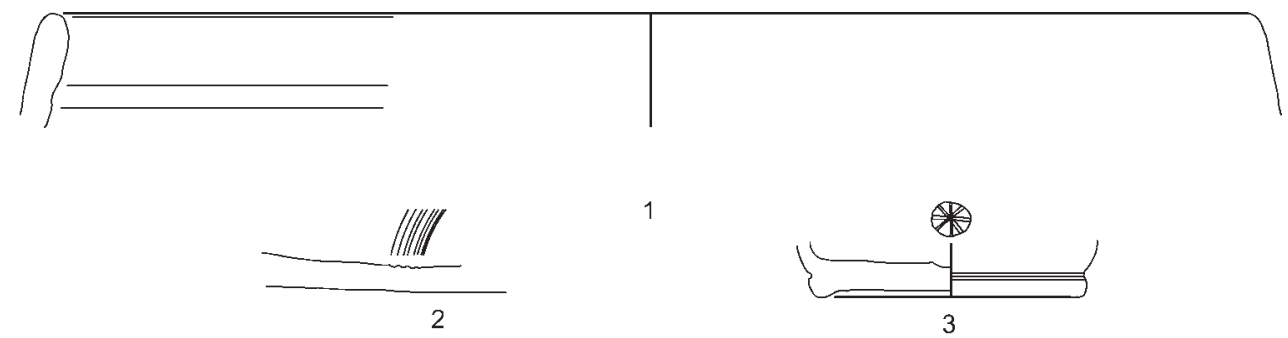

1
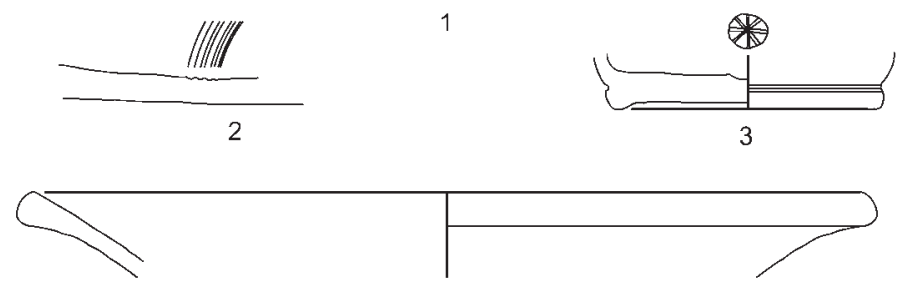

4
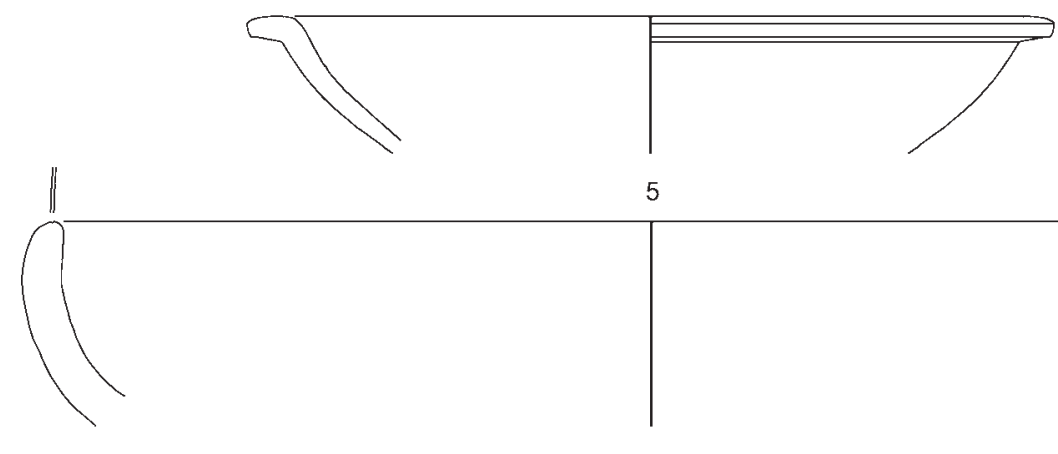

5

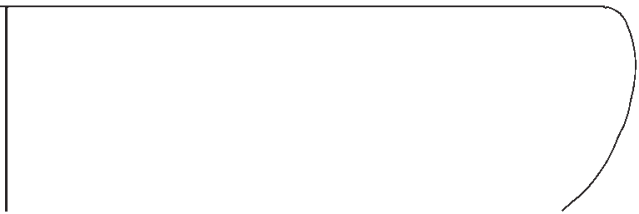

6
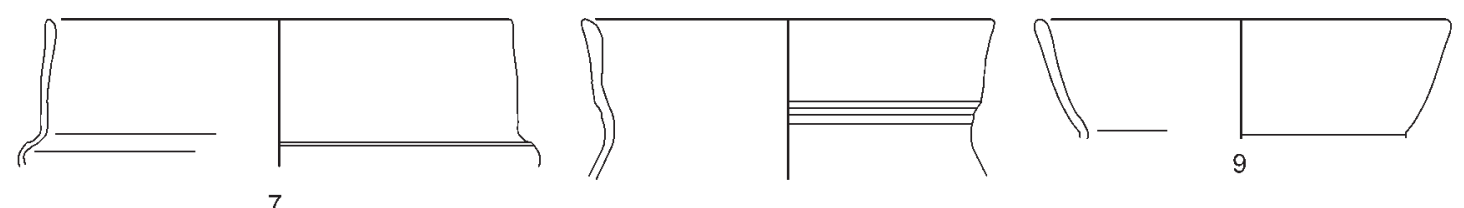

8
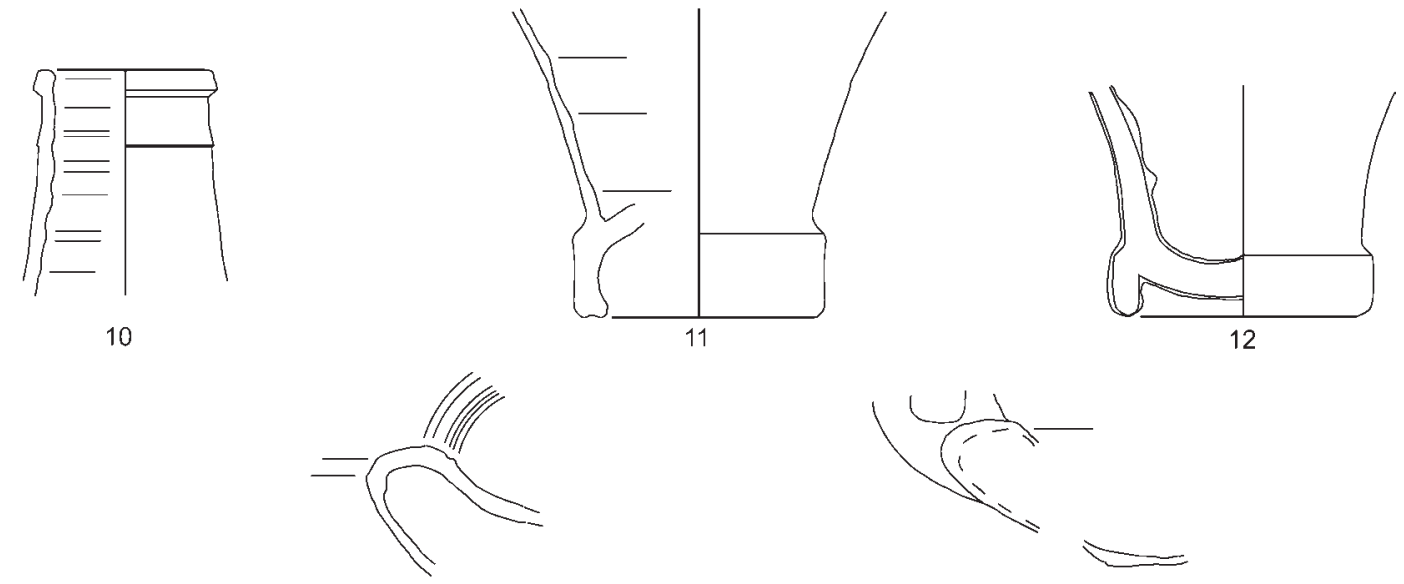

13

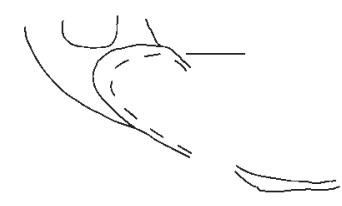

14

SE 35

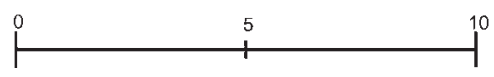

13 Nymphaeum Traiani, Sondage NT 05. Fundauswahl (Kat. 1-14) 
SE 33
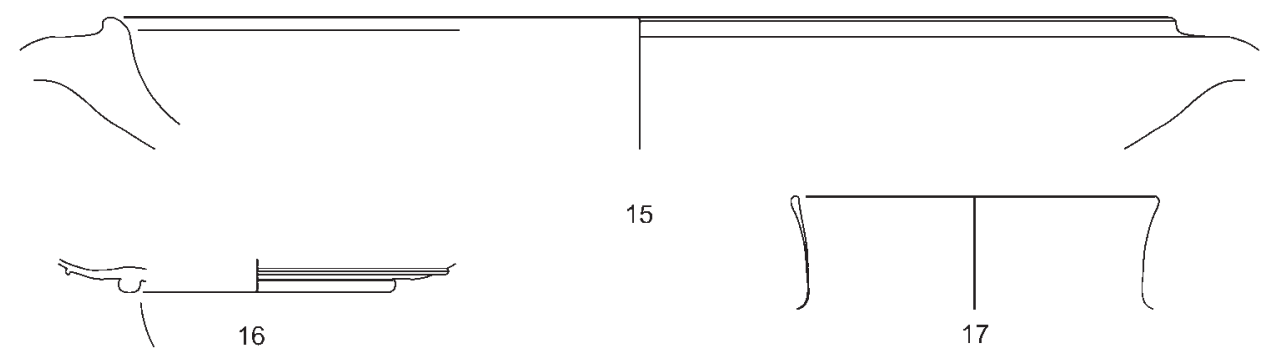

15
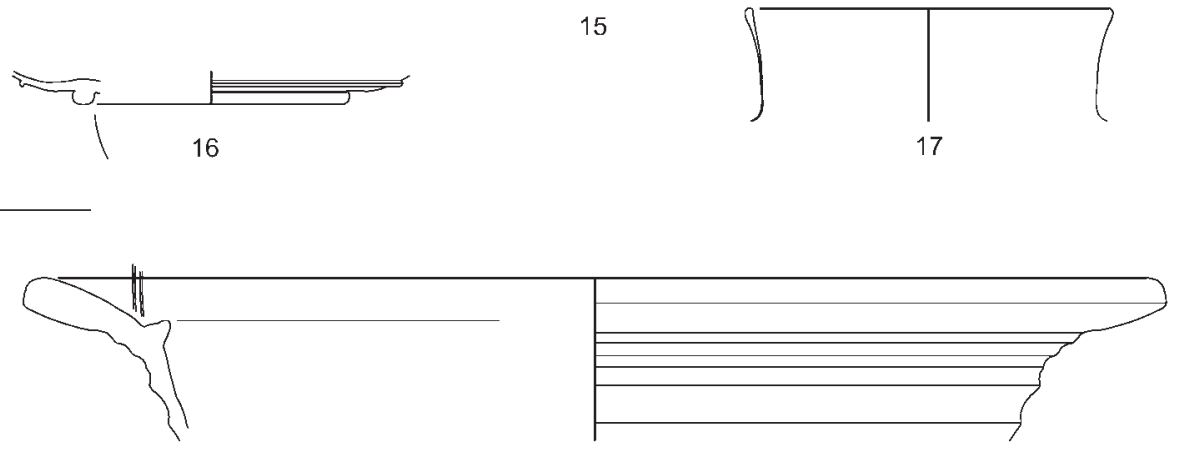

18

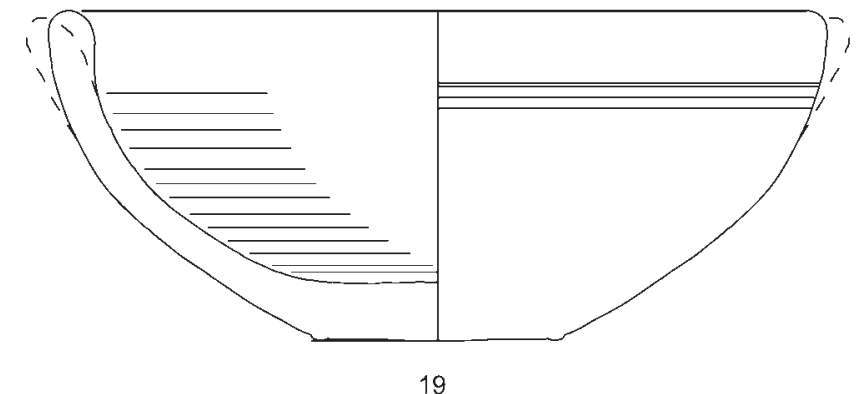

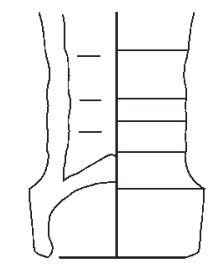

20

SE 22
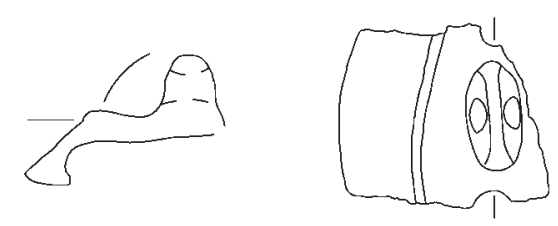

21
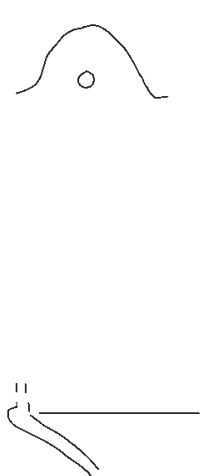

23

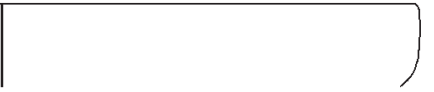

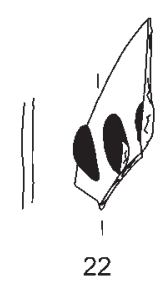

22
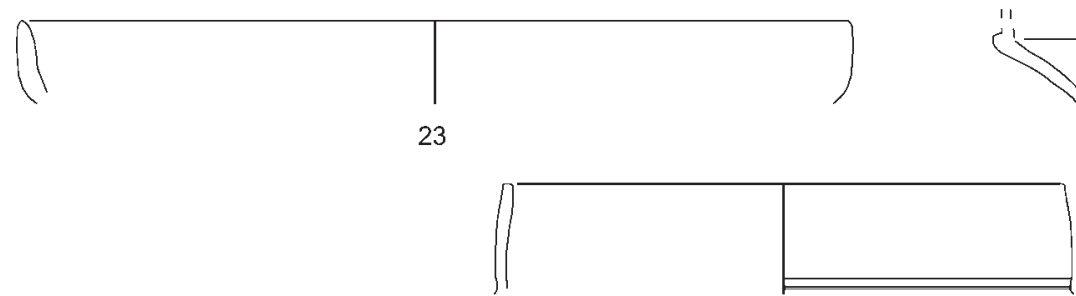

25

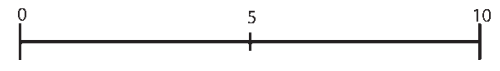

14 Nymphaeum Traiani, Sondage NT 05. Fundauswahl (Kat. 15-25) 

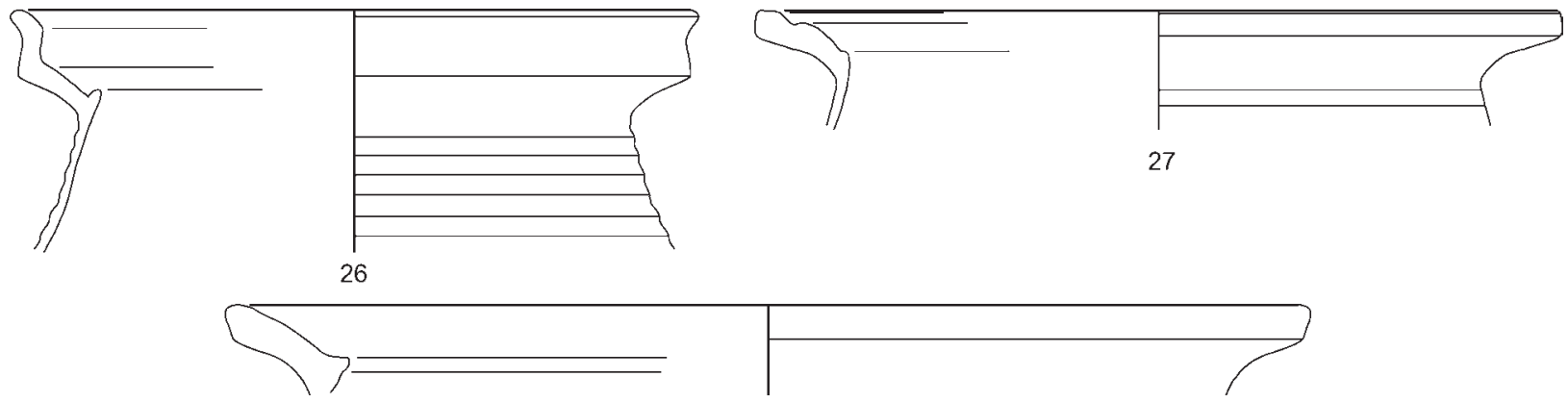

28
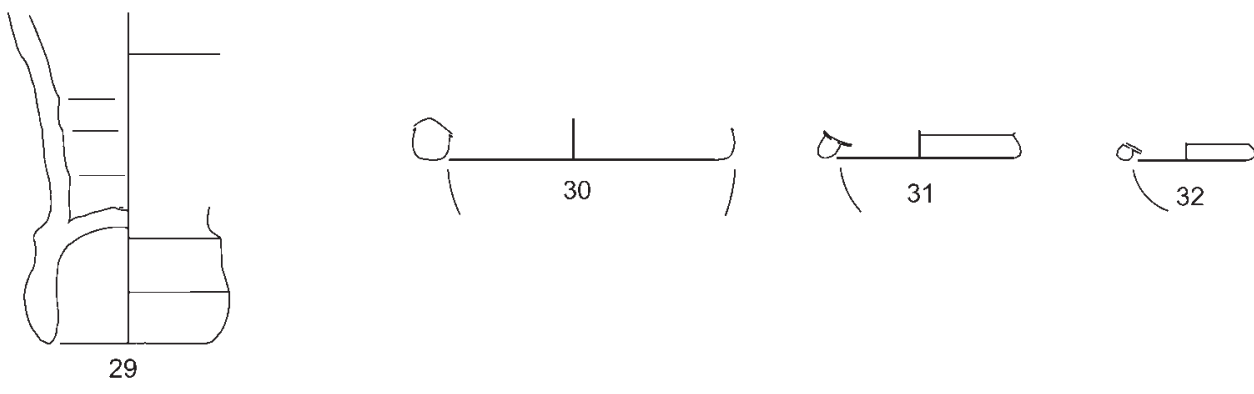

SE 32
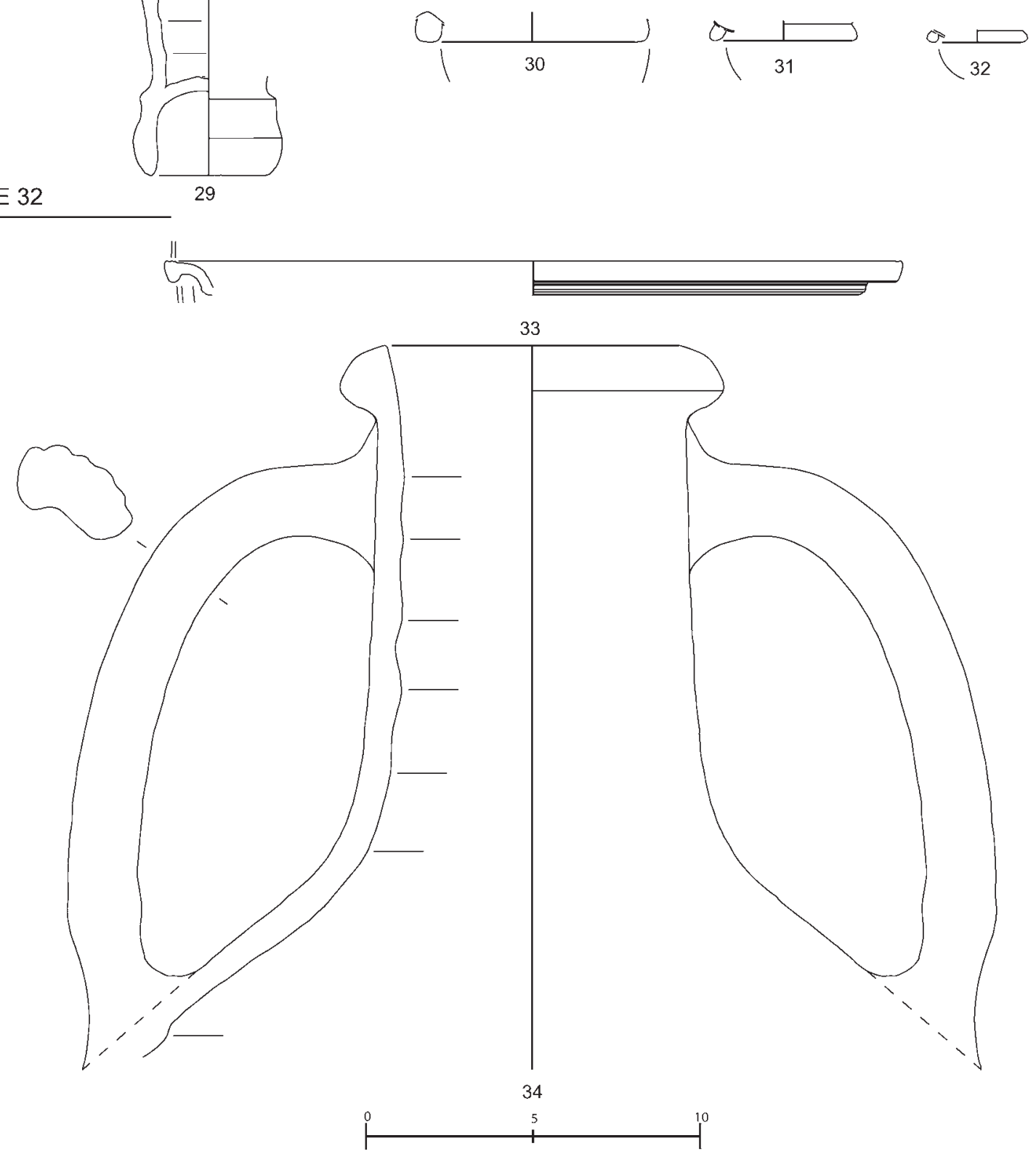

15 Nymphaeum Traiani, Sondage NT 05. Fundauswahl (Kat. 26-34) 

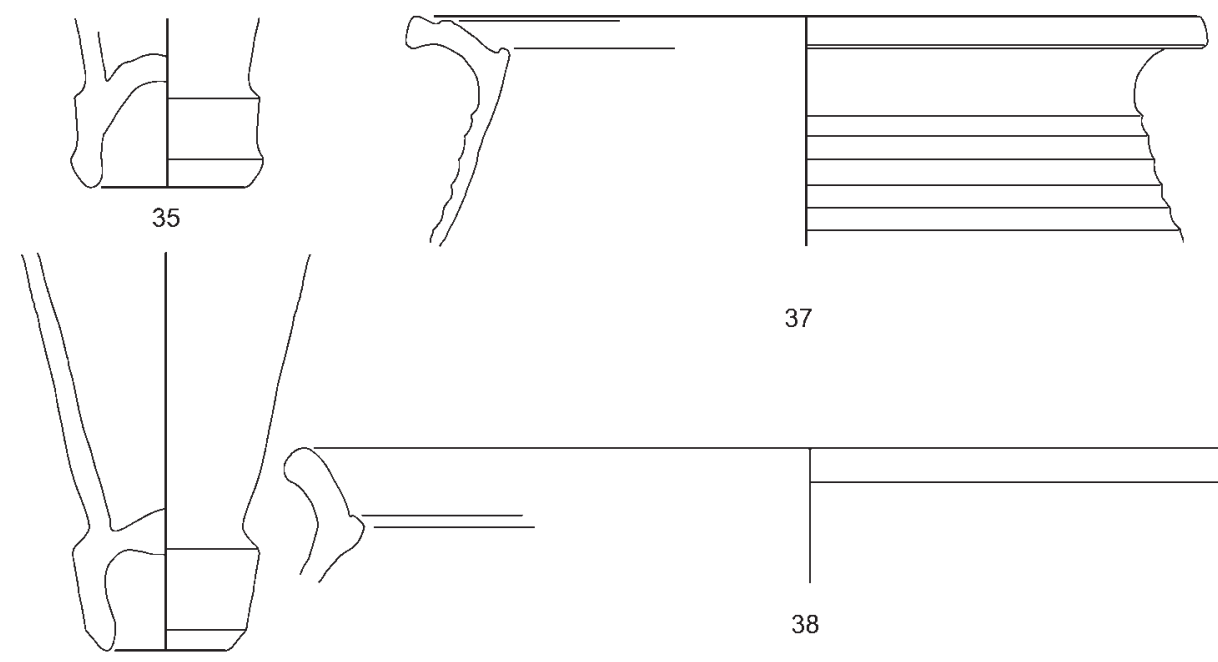

37

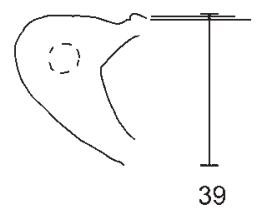

36

SE 17

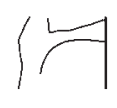

40

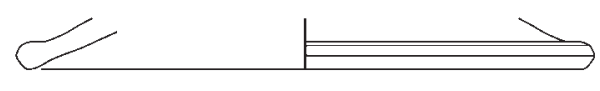

41

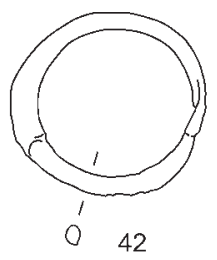

SE 20

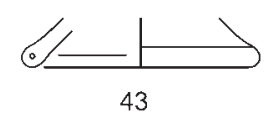

SE 26

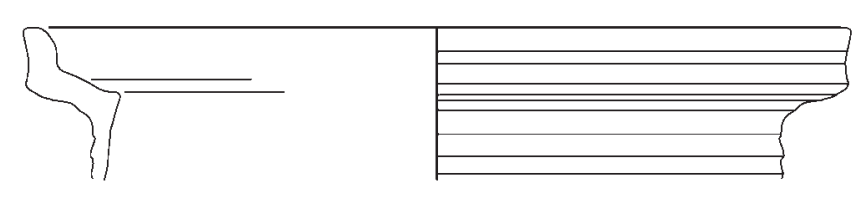

44

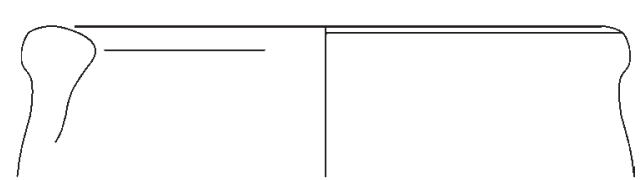

45
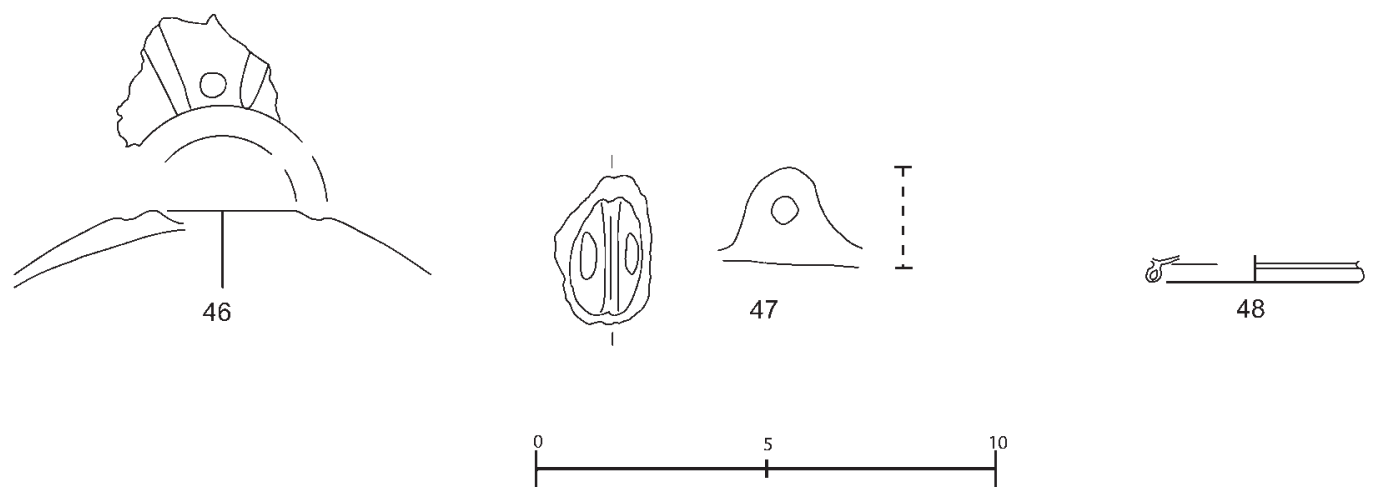

16 Nymphaeum Traiani, Sondage NT 05. Fundauswahl (Kat. 35-48) 


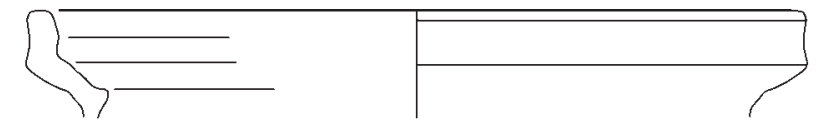

49

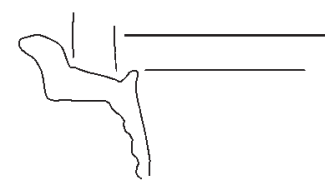

SE 31

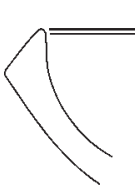

SE 21

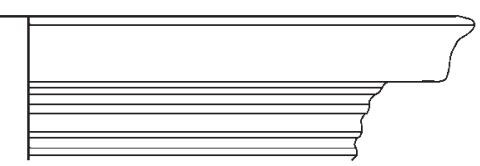

50

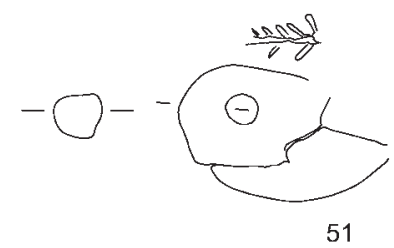

$v=\longmapsto$

52

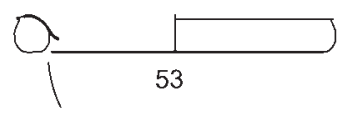

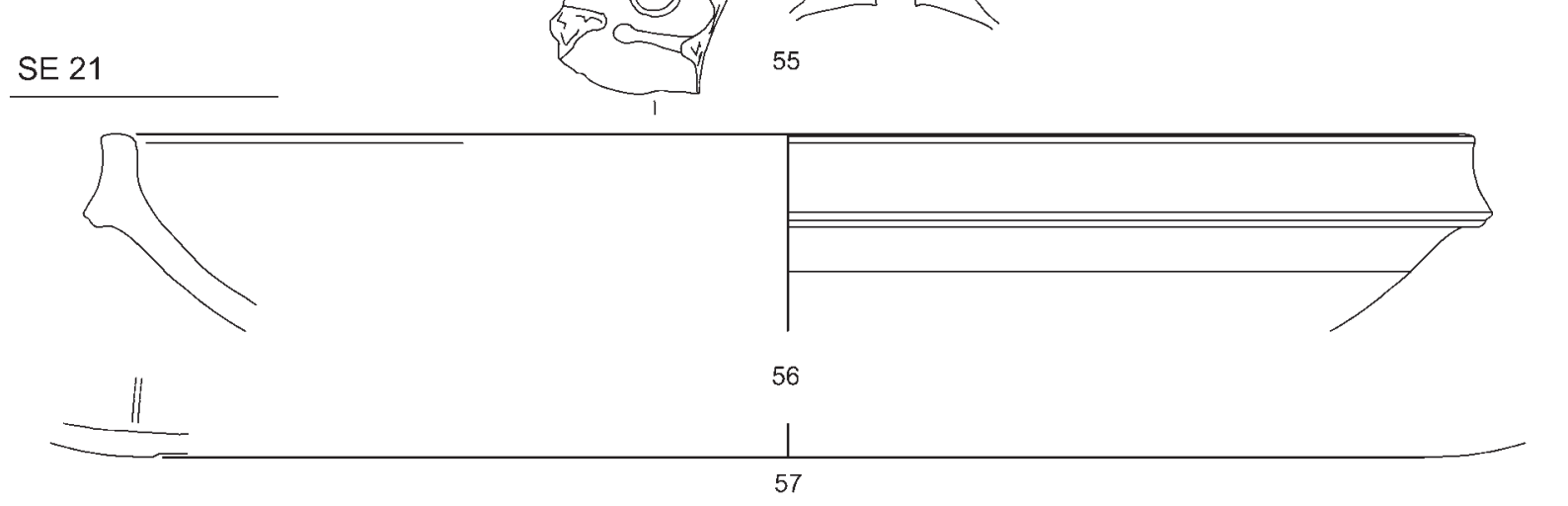

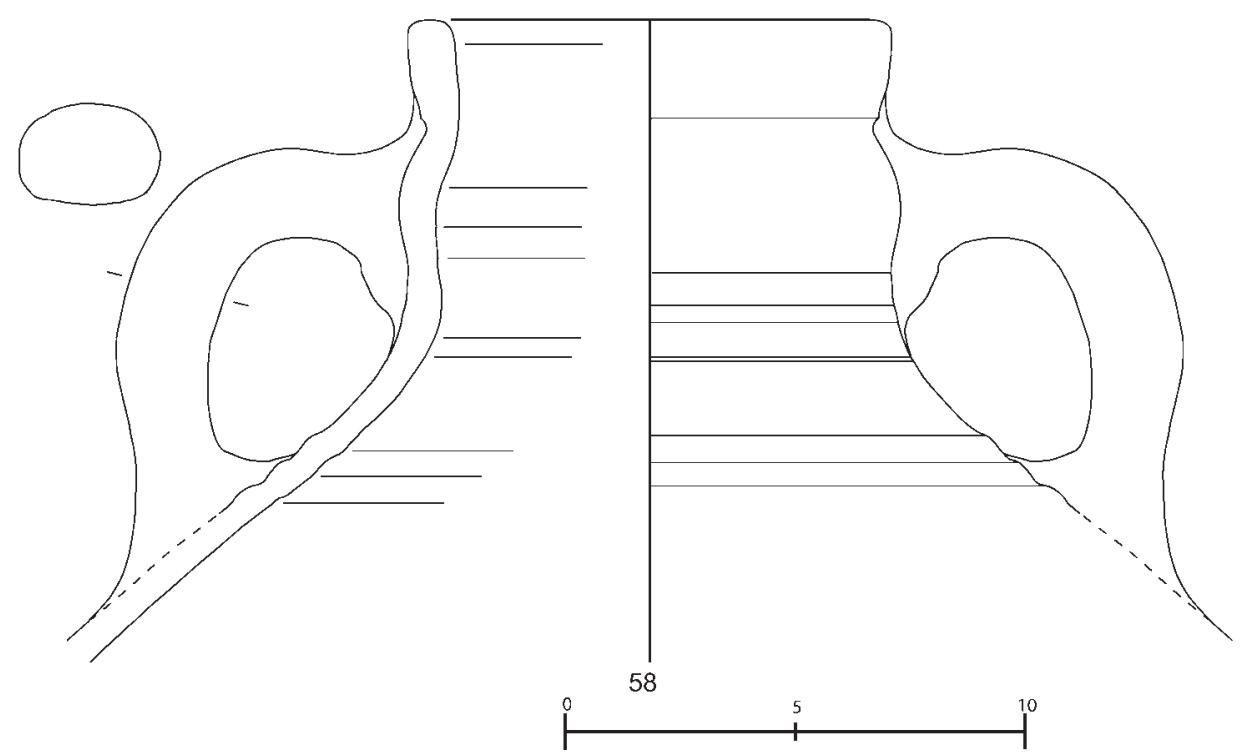

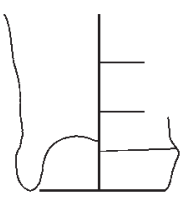

59

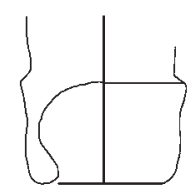

60

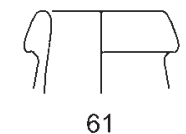

17 Nymphaeum Traiani, Sondage NT 05. Fundauswahl (Kat. 49-61) 

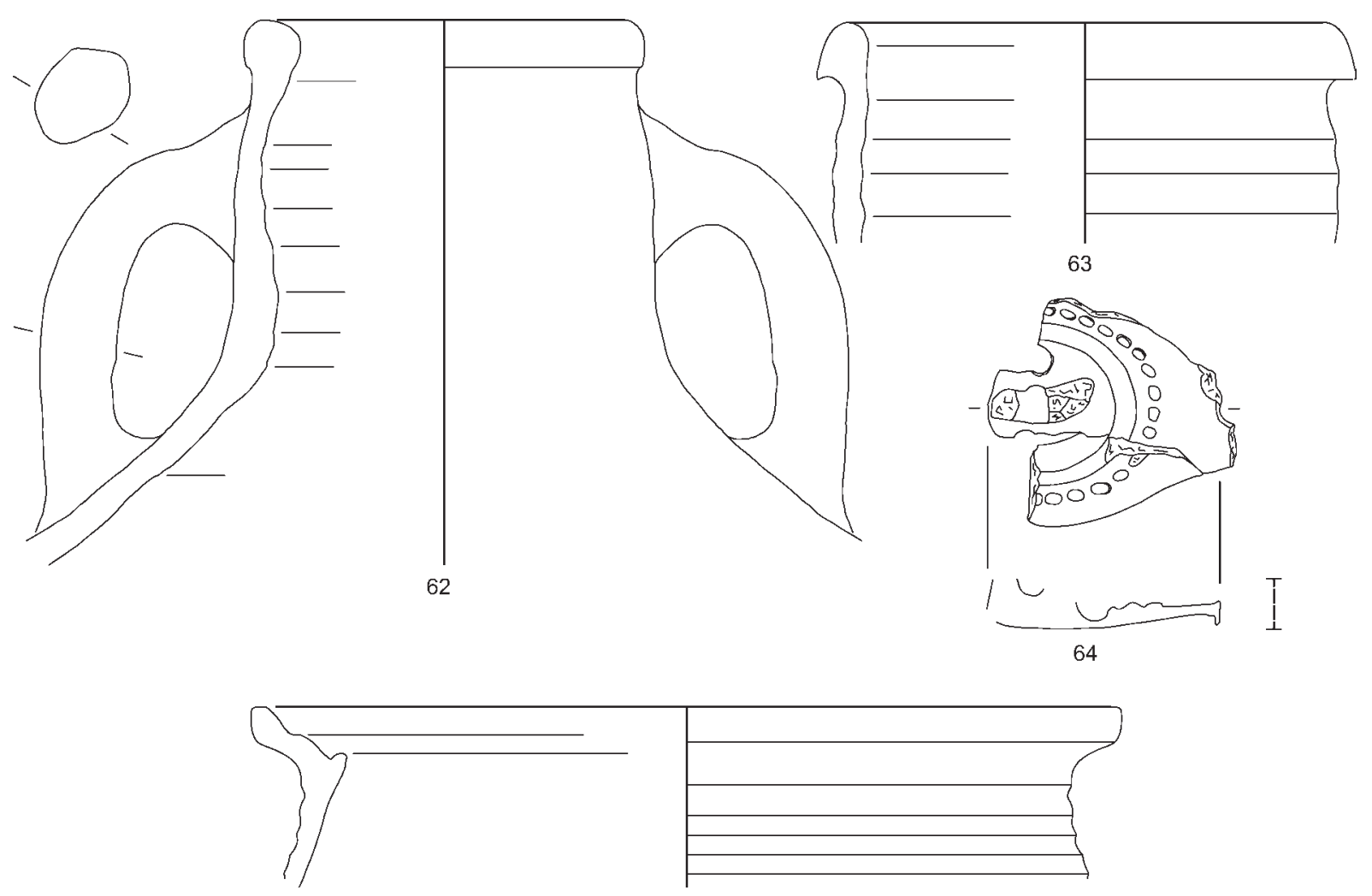

65

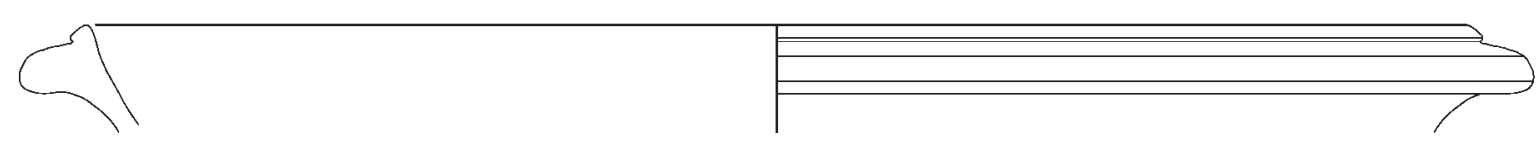

SE 12

66

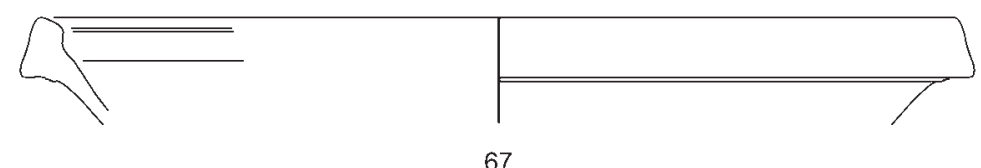

67

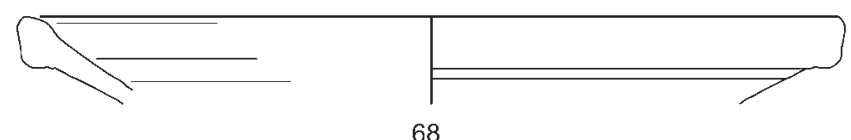

68

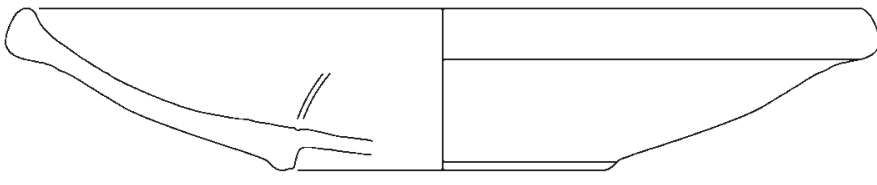

69
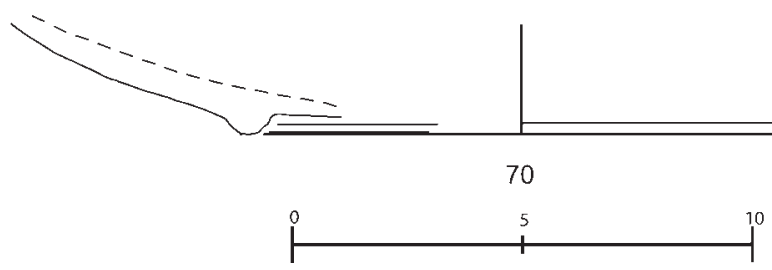

18 Nymphaeum Traiani, Sondage NT 05. Fundauswahl (Kat. 62-71)

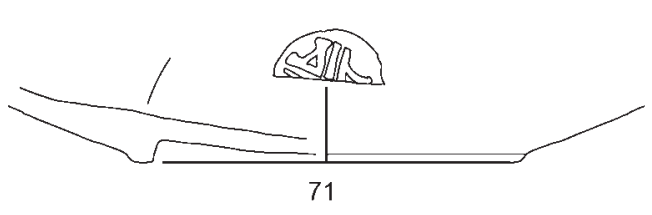



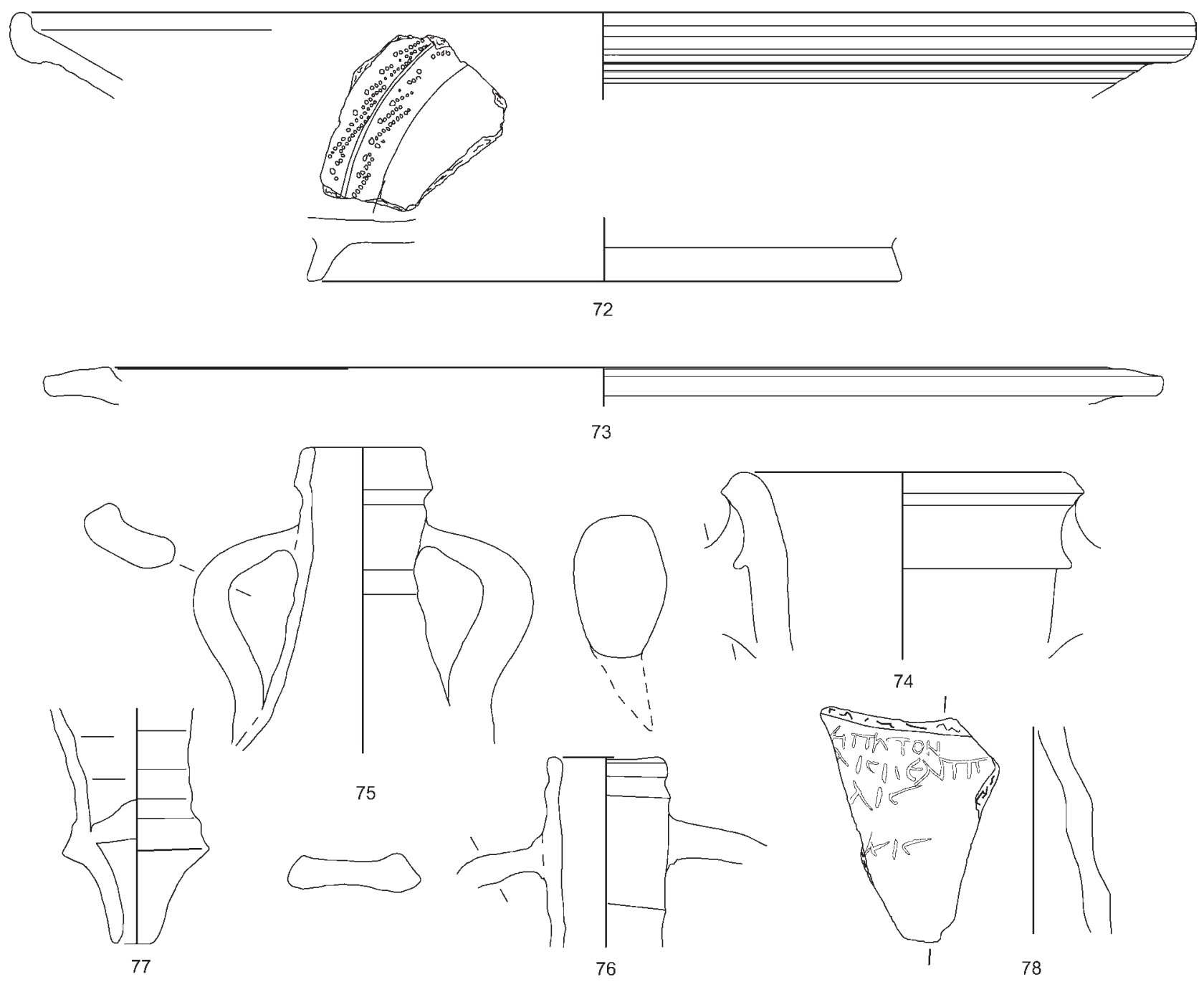

SE 8
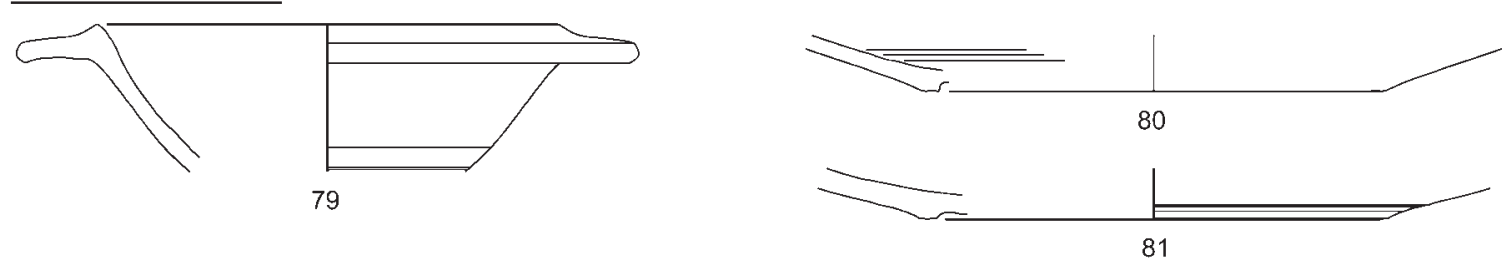

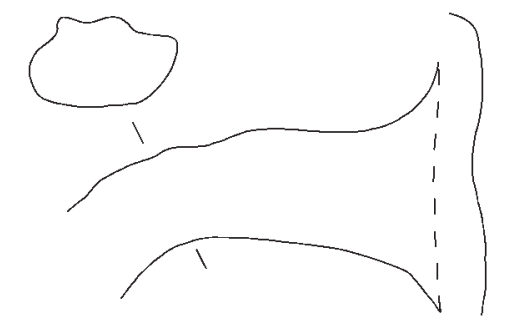

83

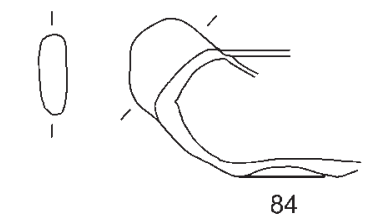

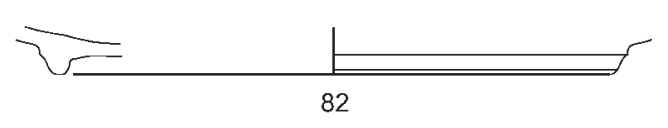

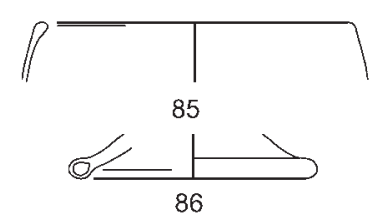

SE 34

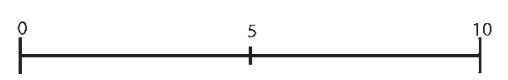

19 Nymphaeum Traiani, Sondage NT 05. Fundauswahl (Kat. 72-86) 


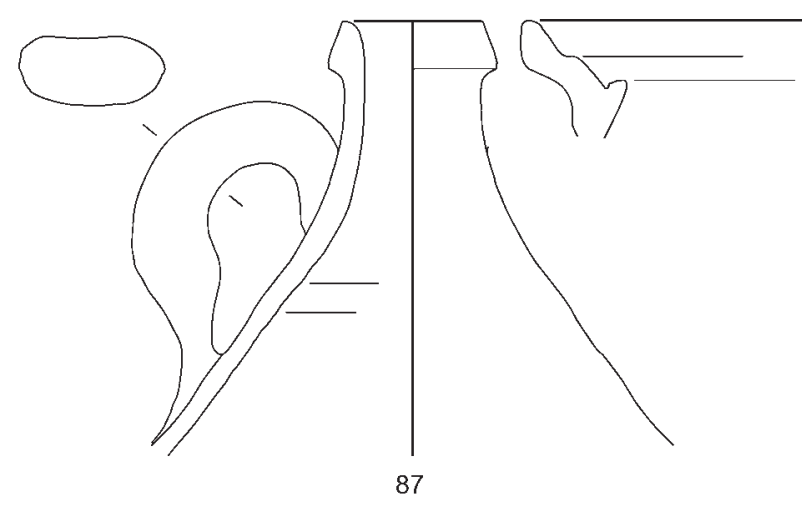

SE 11
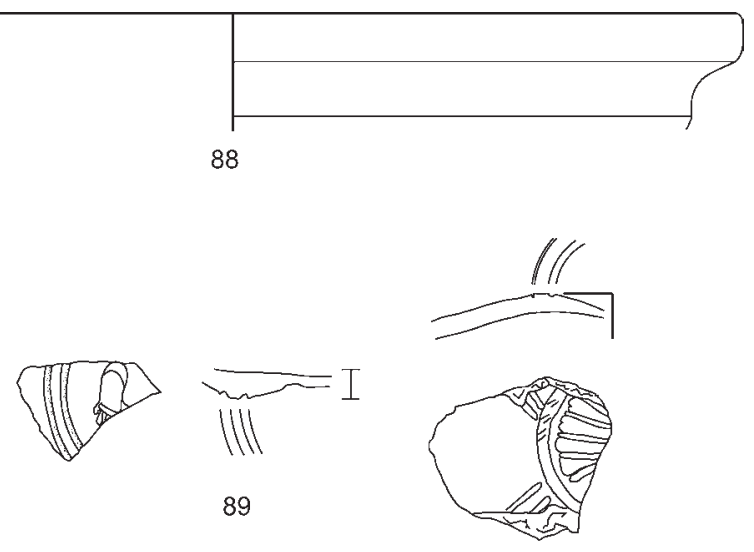

90

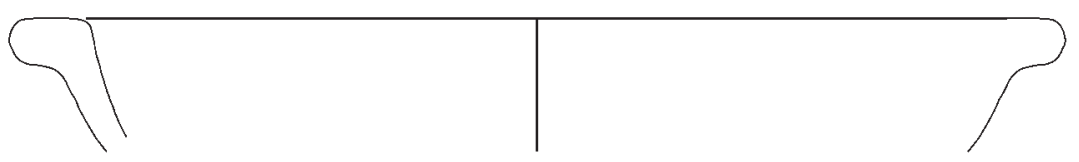

91

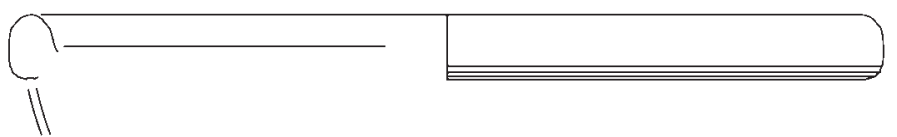

92

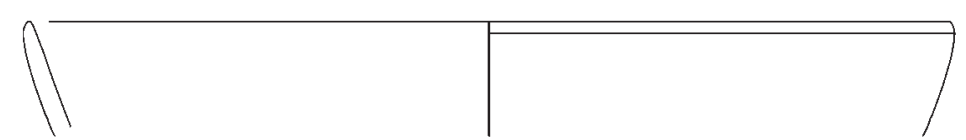

93
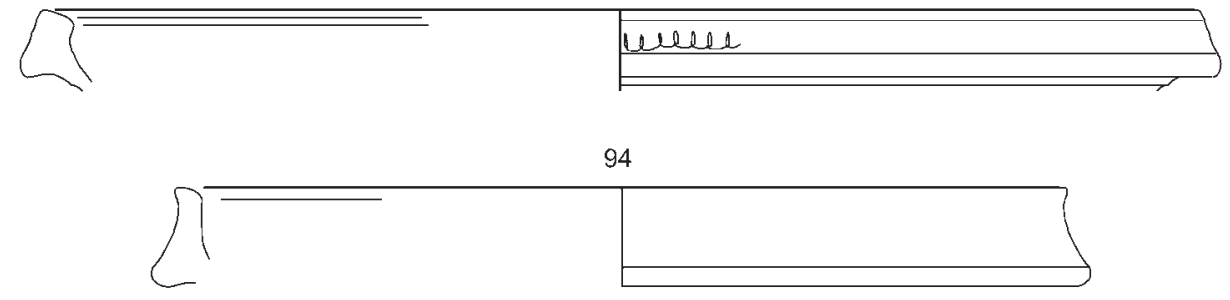

95

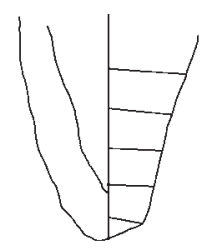

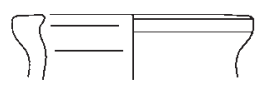

97

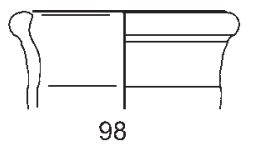

96

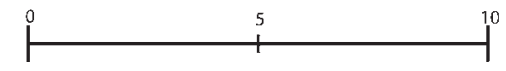

20 Nymphaeum Traiani, Sondage NT 05. Fundauswahl (Kat. 87-98) 


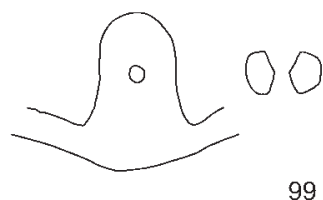

99
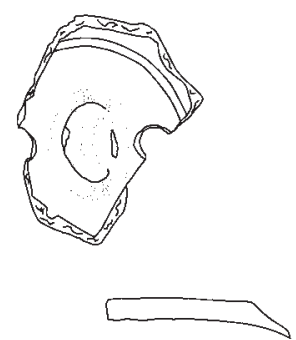

SE 10

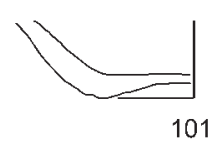

101
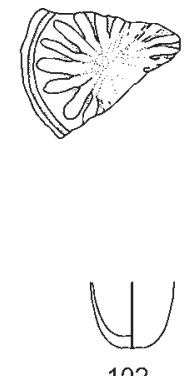

102

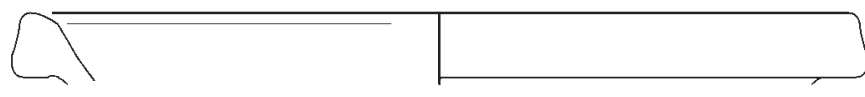

103
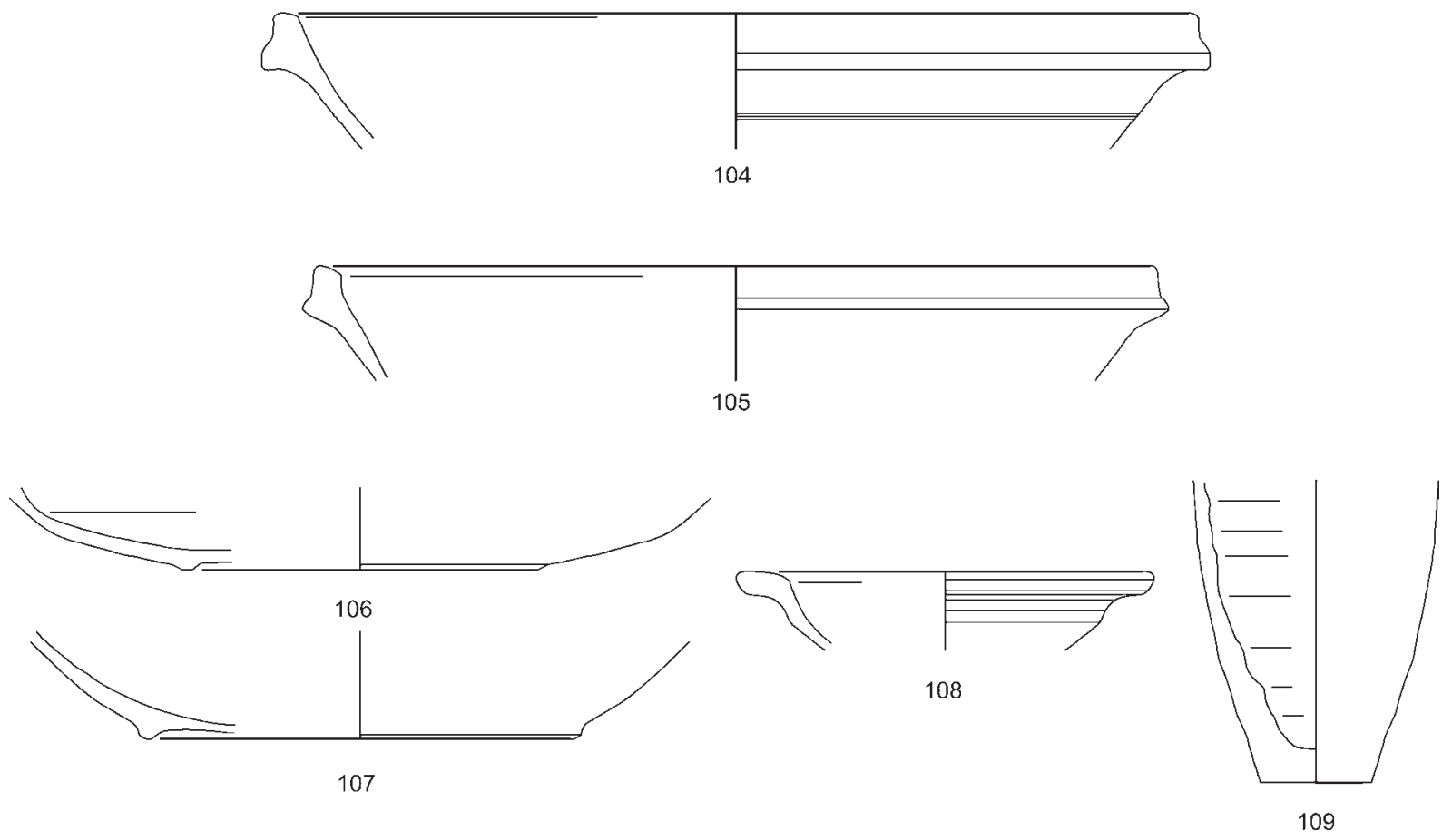

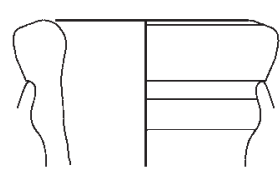

110

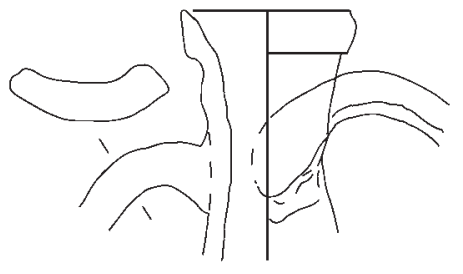

111

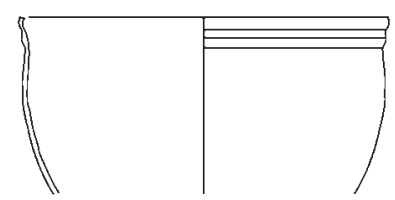

112

SE $7 / 9$

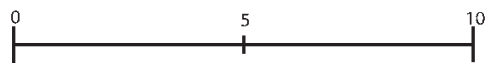

21 Nymphaeum Traiani, Sondage NT 05. Fundauswahl (Kat. 99-112) 


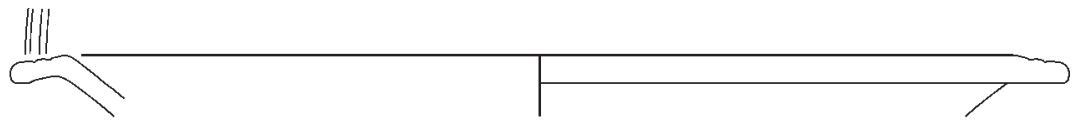

113

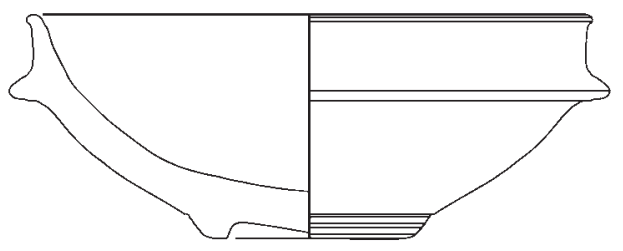

114
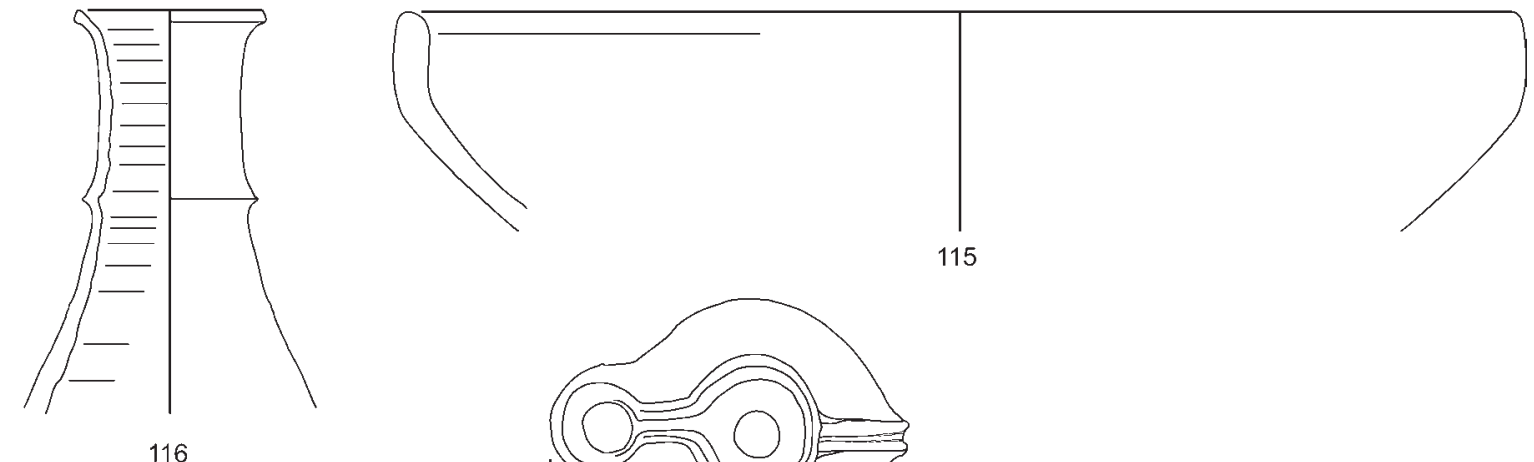

SE 30
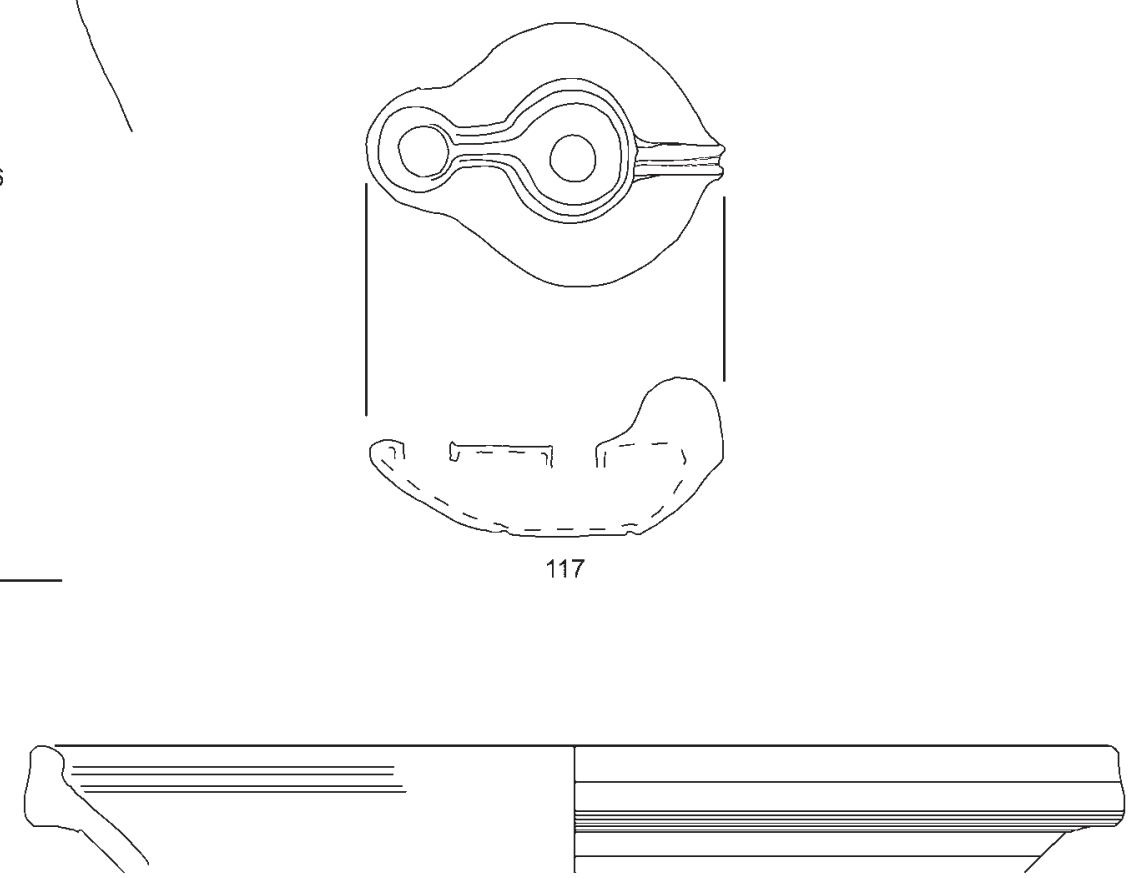

118

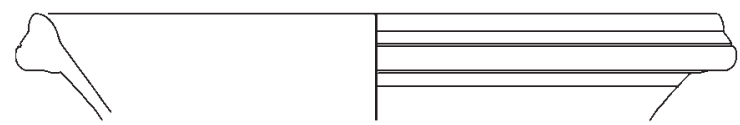

119
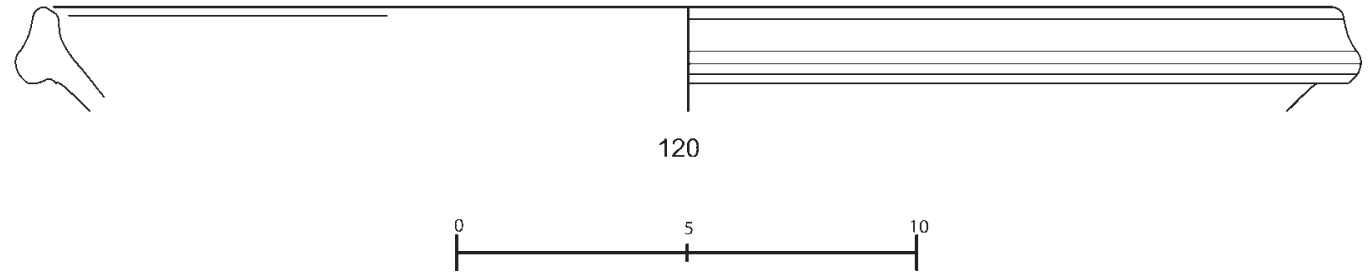

22 Nymphaeum Traiani, Sondage NT 05. Fundauswahl (Kat. 113-120) 

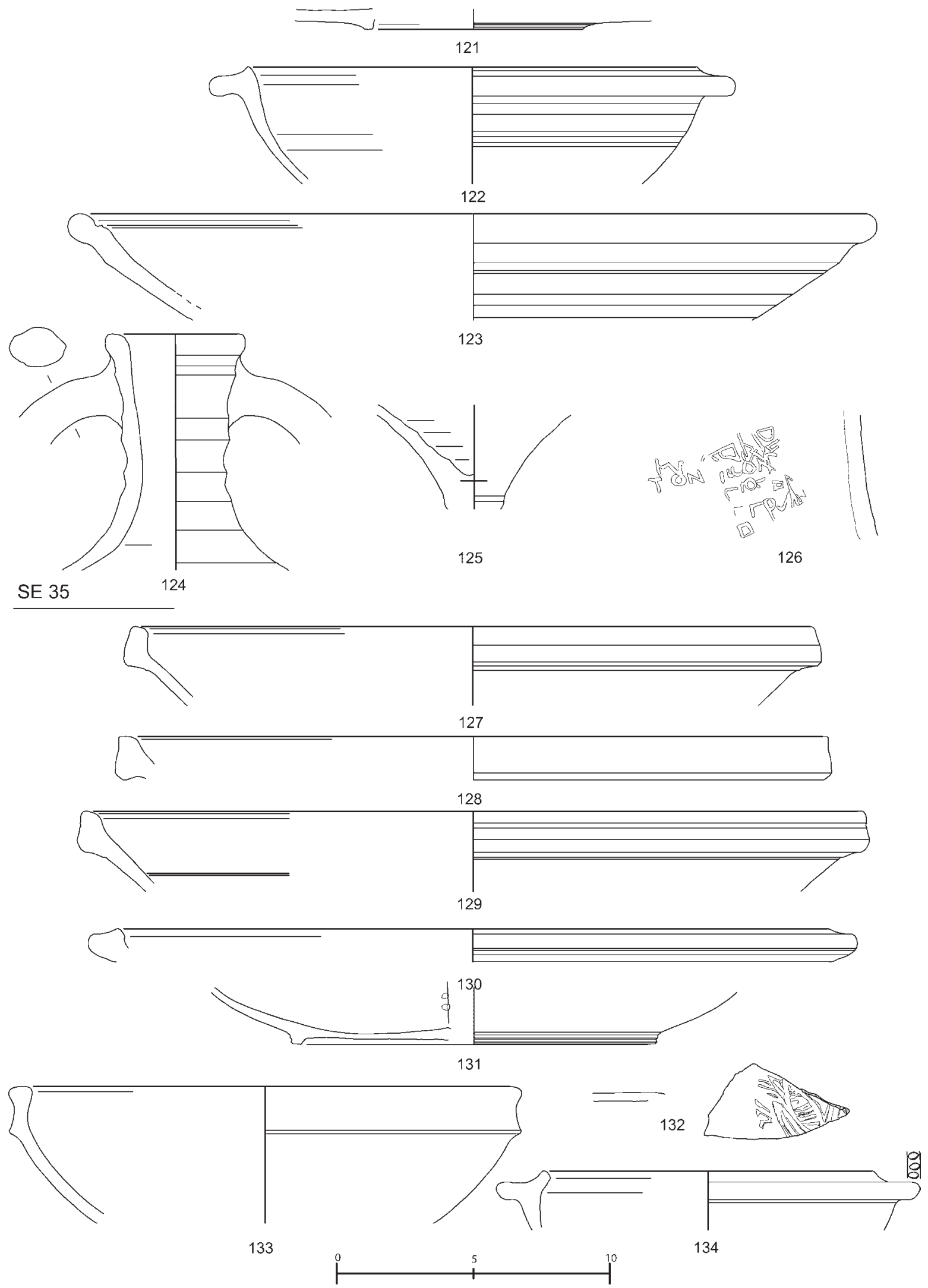

23 Nymphaeum Traiani, Sondage NT 05. Fundauswahl (Kat. 121-134) 


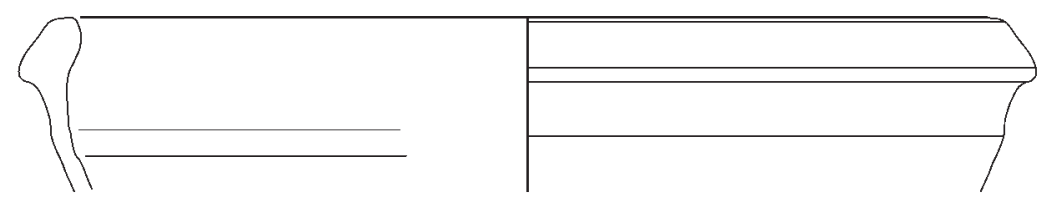

135

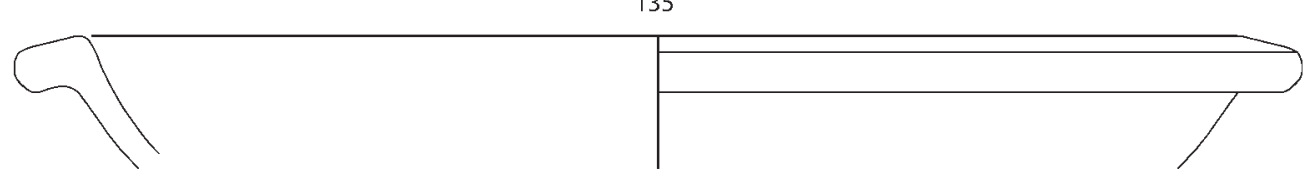

136

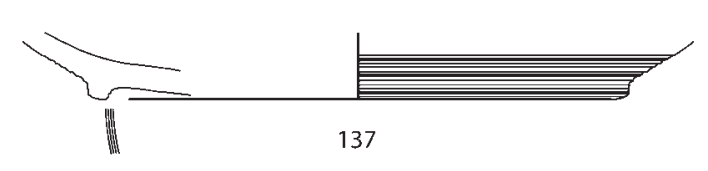

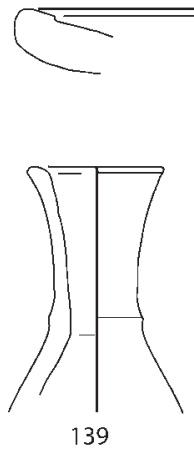

139

SE 1
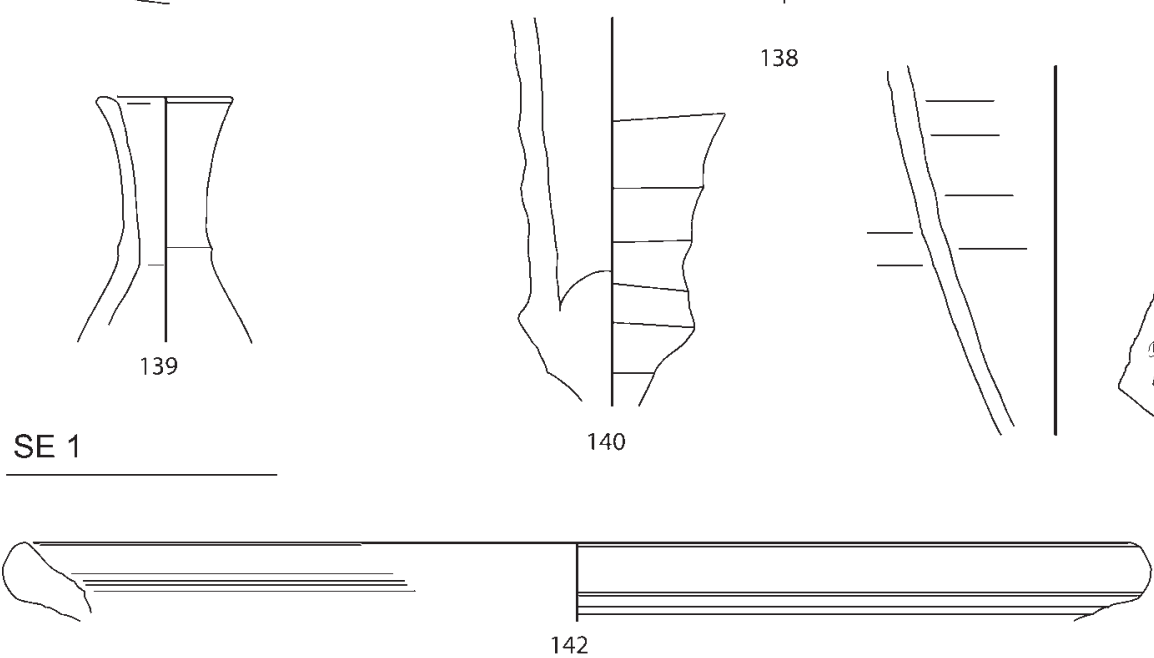

140

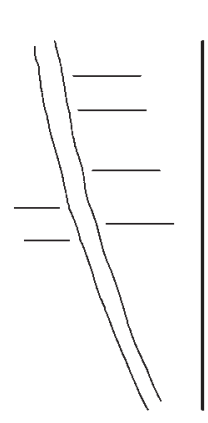

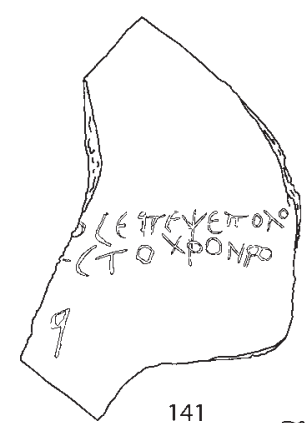

141
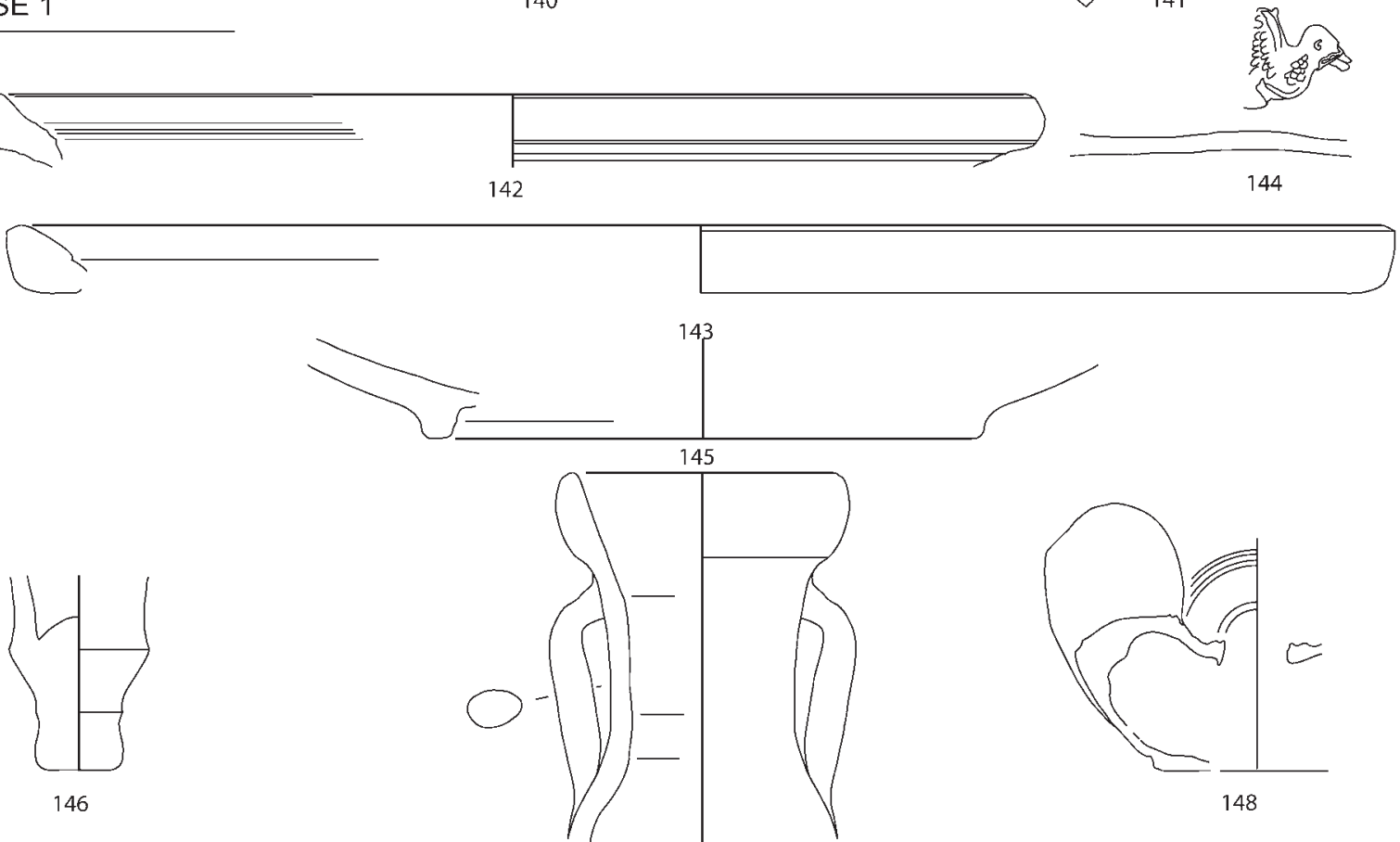

SE $2 / 27$

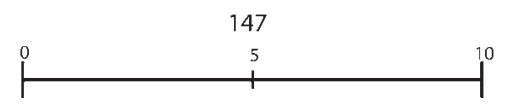

24 Nymphaeum Traiani, Sondage NT 05. Fundauswahl (Kat. 135-148) 

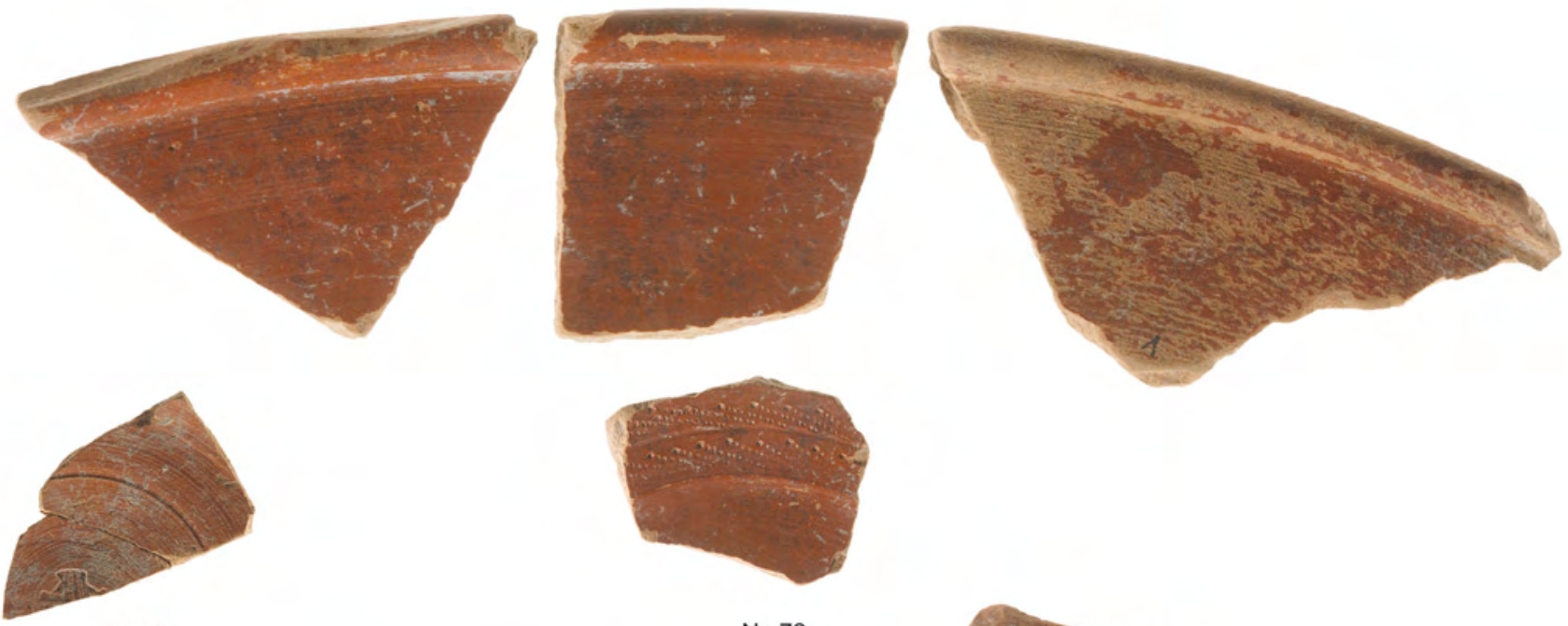

Nr. 71

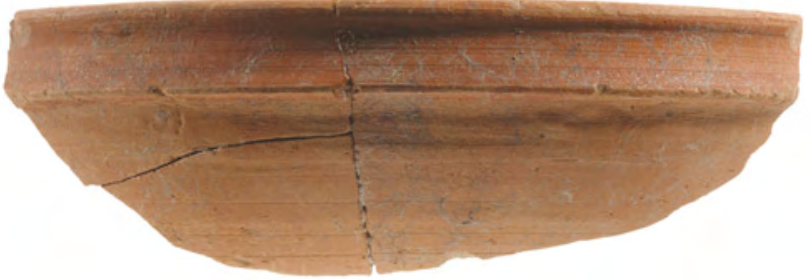

Nr. 133

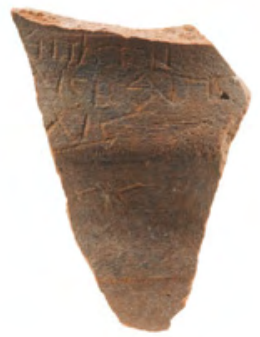

Nr. 78

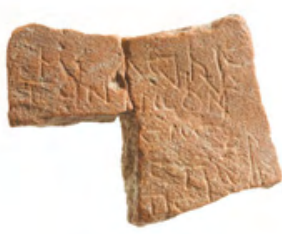

Nr. 127

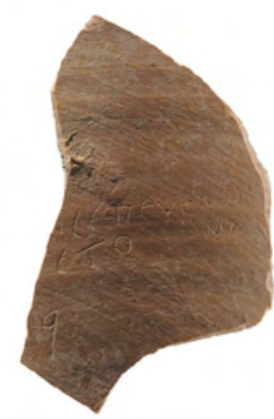

Nr. 141

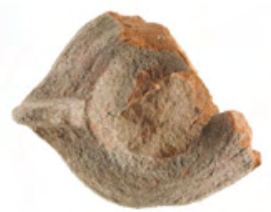

Nr. 84

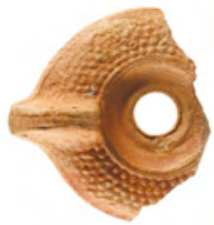

Nr. 148

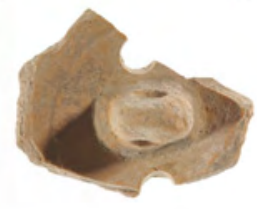

Nr. 21

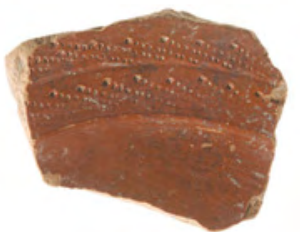

Nr. 72

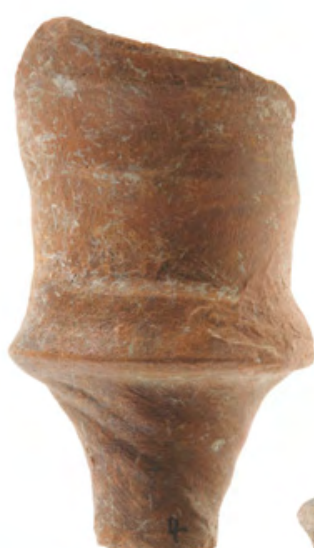

Nr. 77

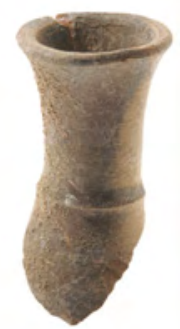

Nr. 116
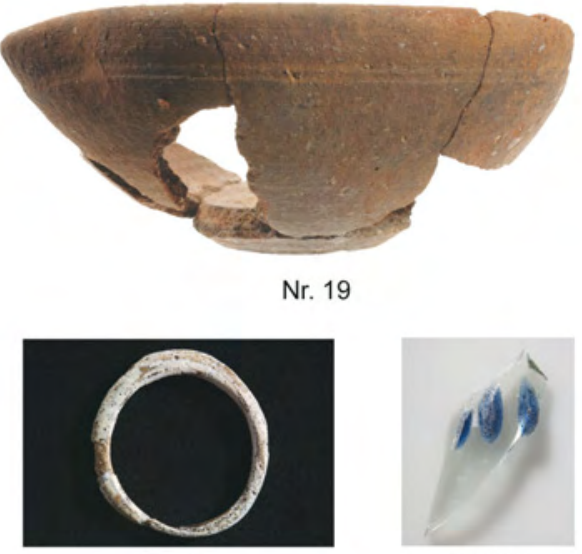

Nr. 42

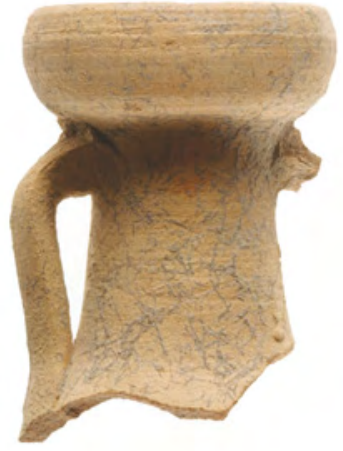

Nr. 147

Nymphaeum Traiani, Sondage NT 05. Ausgewählte Funde 


\section{Fundmünzen ${ }^{186}$}

\begin{tabular}{|c|c|c|}
\hline Mü & Bestimmung & SE \\
\hline $2005 / 4$ & $\begin{array}{l}\text { für Divus Claudius II } \\
\text { Rad; ab 270; Rom oder Nordwestprovinzen; } 6^{\text {h }} ; 1,58 \mathrm{~g} \\
\text { Av.: Leg.?; Stk? } \\
\text { Rv.: Leg.?; Altar }\end{array}$ & 8 \\
\hline $2005 / 5$ & $\begin{array}{l}\text { Unbestimmbar } \\
\text { Fol oder Cen; 4. Jh. n. Chr.; ?h; }(0,68 \text { g) } \\
\text { Fragment }\end{array}$ & 10 \\
\hline $2005 / 6$ & $\begin{array}{l}\text { Unbestimmbar } \\
?^{\text {h}} ;(0,71 \mathrm{~g}) \\
\text { Fragment }\end{array}$ & 10 \\
\hline $2005 / 8$ & $\begin{array}{l}\text { Unbestimmbar } \\
?^{\text {h }} ;(3,82 \mathrm{~g}) \\
\text { stark korrodiert }\end{array}$ & 10 \\
\hline $2005 / 9$ & $\begin{array}{l}\text { Prägeherr unbestimmbar } \\
\text { Mai; } 348-355 \text { n. Chr.; Ms.?; 6 } \text {; }(0,74 \text { g) } \\
\text { Av.: ...]AVG; Büste r. } \\
\text { Rv.: Leg.?; Typ fel temp reparatio (Reitersturz) }\end{array}$ & 7 \\
\hline $2005 / 10$ & $\begin{array}{l}\text { Constantius II } \\
\text { Cen; } 351-355 \mathrm{n} . \text { Chr.; Con; } 6^{\text {h; }} 2,02 \mathrm{~g} \\
\text { Av.: DNCONSTAN-TIVSPFAVG; PDiad2PCv } \\
\text { Rv.: FELTEMPRE-PARATIO, - -//CONSA; Reitersturz } \\
\text { RIC } 118\end{array}$ & 11 \\
\hline $2005 / 13$ & $\begin{array}{l}\text { Constantius II } \\
\text { Cen; } 351-361 \mathrm{n} . \text { Chr.; Ms.?; 5 } ;(1,51 \mathrm{~g}) \\
\text { Av.: Leg. ?; PDiad2PCv } \\
\text { Rv.: Leg.?; Reitersturz } \\
\text { Fragment }\end{array}$ & 7 \\
\hline $2005 / 14$ & $\begin{array}{l}\text { Unbestimmbar } \\
?^{\text {h }} ;(0,71 \mathrm{~g}) \\
\text { Fragment }\end{array}$ & 12 \\
\hline $2005 / 15$ & $\begin{array}{l}\text { Constantius II } \\
\text { Cen; } 351-361 \mathrm{n} . \text { Chr.; Ms.?; } 6^{\text {h}} ;(1,51 \mathrm{~g}) \\
\text { Av.: Leg.?; PDiad2PCv } \\
\text { Rv.: Leg.?; Reitersturz } \\
\text { Ausbrüche }\end{array}$ & 12 \\
\hline $2005 / 20$ & $\begin{array}{l}\text { Theodosius I } \\
1 / 2 \text { Cen; } 388-395 \text { n. Chr.; Con; } 1^{\text {h}} ; 1,08 \mathrm{~g} \\
\text { Av.: DNTHEODO-SIVSPFAVG; PDiad2PCv } \\
\text { Rv.: SALVSREI-PVBLICAE, Chrismon -//CONSA; Victoria schleppt Gefangenen n. } 1 . \\
\text { RIC } 86 \mathrm{~b}=88 \mathrm{a}\end{array}$ & 17 \\
\hline $2005 / 21$ & $\begin{array}{l}\text { Constantius II oder Constans I } \\
\text { Fol; } 341-348 \mathrm{n} . \text { Chr.; Nic; } 12^{\mathrm{h}} ; 1,59 \mathrm{~g} \\
\text { Av.: Leg.?; Büste r. } \\
\text { Rv.: VOT/XX/MVLT/XXX in Kranz, - -SMN?([) } \\
\text { LRBC 1149. 1150. } 1156 \text { oder } 1157\end{array}$ & 17 \\
\hline $2005 / 22$ & $\begin{array}{l}\text { Constantius II } \\
\text { Cen; } 350-355 \text { n. Chr.; Tes; } 6^{\text {h}} ; 2,06 \mathrm{~g} \\
\text { Av.: DNCONSTAN-TIVSPFAVG; PDiad2PCv } \\
\text { Rv.: FELTEMP-REPARATIO, } \Gamma \text {-//SMTS; Reitersturz FH3 }\end{array}$ & 17 \\
\hline
\end{tabular}

${ }^{186}$ Aus dem Gesamtmaterial wurden die für eine kontextuelle Auswertung relevanten Fundmünzen der Grabung 2005 ausgewählt. Die in diesem Abschnitt verwendeten Abkürzungen folgen den Regeln der Wiener Schule, wie sie in F. Schmidt-Dick, Die römischen Münzen des Medagliere im Castelvecchio zu Verona, Thesaurus Nummorum Romanorum et Byzantinorum IX (Wien 1995) 15-18 festgelegt sind. 


\begin{tabular}{|c|c|c|}
\hline $2005 / 24$ & $\begin{array}{l}\text { Constantius II } \\
\text { Mai; } 351-354 \text { n. Chr.; Kyz; 12h; 3,69 g } \\
\text { Av.: DNCONSTAN-TIVSPFAVG; PDiad2PCv } \\
\text { Rv.: FELTEMPRE-PARATIO, } \Gamma \text {-//@SMKГ } \\
\text { RIC } 95\end{array}$ & 22 \\
\hline $2005 / 25$ & $\begin{array}{l}\text { Constantius II } \\
\text { Fol; } 341-348 \text { n. Chr.; Ms.?; } 7^{\text {h. }} \text { 0,89 g } \\
\text { Av.: Leg.? (nach Resten Constantius II); PDiad2 } \\
\text { Rv.: VOT/XX/MVLT/XXX in Kranz, Sigle? }\end{array}$ & 26 \\
\hline $2005 / 30$ & $\begin{array}{l}\text { Arcadius, Honorius oder Theodosius II } \\
\text { 1/2 Cen; 404-406 n. Chr.; Ms.?; 2 } ; 0,68 \mathrm{~g} \\
\text { Av.: Leg.?; Büste r. } \\
\text { Rv.: Leg.?; Kreuz (Typ concordia aug) }\end{array}$ & 41 \\
\hline $2005 / 31$ & $\begin{array}{l}\text { Honorius } \\
\text { Cen; 395-401 n. Chr.; Ms.?; } 12^{\text {h }} ; 2,20 \mathrm{~g} \\
\text { Av.: .]NH[...]VSPF[...; PDiad2PCV } \\
\text { Rv.?: Leg. und Sigle unkenntlich; Kaiser 1. stehend, von Victoria bekränzt (Typ virtus exerciti) }\end{array}$ & 1 \\
\hline $2005 / 34$ & $\begin{array}{l}\text { Valentinianus II } \\
\text { 1/2 Cen; 388-395 n. Chr.; Con; 7 } ; 0,97 \mathrm{~g} \\
\text { Av.: DNVALENTINIANV[...; PDiad2PCV } \\
\text { Rv.: Leg.?, Chrismon -//SM[; Victoria schleppt Gefangenen n. 1. (Typ salus rei publicae) }\end{array}$ & 11 \\
\hline $2005 / 35$ & $\begin{array}{l}\text { Constantius II } \\
\text { Cen; } 351-361 \text { n. Chr.; Con; } 12^{\mathrm{h}} ; 1,87 \mathrm{~g} \\
\text { Av.: DNCONSTAN-TIVSPFAVG; PDiad2PCv } \\
\text { Rv.: FELTEMPRE-PARATIO, - -//CONS[; Reitersturz FH3 } \\
\text { RIC } 118.125 \text { oder } 135\end{array}$ & 12 \\
\hline $2005 / 36 \mathrm{~A}$ & $\begin{array}{l}\text { Constantius II } \\
\text { Cen; 351-361 n. Chr.; Ms.?; 5 } ; \text { (0,87 g) } \\
\text { Av.: Leg.? (nach Resten Constantius II); ?Diad?PCv } \\
\text { Rv.: Leg. und Sigle unkenntlich; Reitersturz FH? } \\
\text { Fragment } \\
\end{array}$ & $\wedge 1$ \\
\hline $2005 / 36 \mathrm{~B}$ & $\begin{array}{l}\text { Constantius II } \\
\text { Cen; 351-361 n. Chr.; Ms.?; 6' (0,53 g) } \\
\text { Av.: Leg.? (nach Resten Constantius II); PDiad?PCv } \\
\text { Rv.: Leg. und Sigle unkenntlich; Reitersturz FH? } \\
\text { Randausbrüche }\end{array}$ & 12 \\
\hline $2005 / 39$ & $\begin{array}{l}\text { Unbestimmbar } \\
\text { Cen; ?h: }(0,40 \mathrm{~g}) \\
\text { Av.: Leg.?; PDiad?Büste? } \\
\text { Rv.: Unkenntlich } \\
\text { Fragment } \\
\end{array}$ & 26 \\
\hline $2005 / 46$ & $\begin{array}{l}\text { Gallienus } \\
\text { An; 260-268 n. Chr.; Ms.?; 6 } ; 1,13 \text { g } \\
\text { Av.: Leg.?; Stk } \\
\text { Rv.: Leg.?, H -//-; stehende Figur }\end{array}$ & 32 \\
\hline $2005 / 47$ & $\begin{array}{l}\text { Ephesos: Iulia Domna } \\
\text { AE; 193-217 n. Chr.; Eph; 2 } 2^{\text {h. } 3,25 g} \\
\text { Av.: Leg.?; Büste r. } \\
\text { Rv.: EФE [...; Darstellung unkenntlich }\end{array}$ & 35 \\
\hline $2005 / 48$ & $\begin{array}{l}\text { Unbestimmbar } \\
\text { ?h; 4,35 g }\end{array}$ & 35 \\
\hline
\end{tabular}




\section{Funde aus Marmor: Skulptur und Mobiliar ${ }^{187}$}

Der herausragende Fund dieser Grabung ist die Kopfreplik des Doryphoros ${ }^{188}$ des Polyklet (S 1 Abb. 26-31). Dieser ist Teil des im späten 6./frühen 7. Jahrhundert umgelagerten Werkstattschutts und stammt vielleicht von einer Kalkbrennerei ${ }^{189}$.

Die Abmessungen des Kopfes liegen im Rahmen der Maße, die an anderen Repliken genommen wurden ${ }^{190}$. Kopf- sowie Gesichtshöhe und Details wie die Mundbreite oder der Abstand der äußeren Augenwinkel entsprechen denen der Bronzeherme des Apollonios ${ }^{191}$.

Der Kopf - aus grobkörnigem, weißem Marmor mit grauen Adern gearbeitet - ist etwa in Kinnhöhe abgeschlagen. Sein Erhaltungszustand ist gut, abgesehen von der beschädigten mittleren Partie des Gesichts und kleineren Bestoßungen. Das Inkarnat des Kopfes ist geglättet, die einzelnen Locken sind an allen Seiten ausgearbeitet und - mit graduellen Unterschieden - durch Binnenzeichnung gestaltet.

Der Kopf ist zu seiner Rechten gewendet und geneigt; dabei war er offenbar etwas stärker geneigt als an der Apolloniosherme und an den Statuenrepliken in Minneapolis und Neapel ${ }^{192}$. Eine Reihe von Details der Ausarbeitung, die auf den Kopisten zurückgehen, nimmt auf die Wendung des Kopfes und dessen Ansichtsseite Rücksicht: Das rechte Auge liegt etwas tiefer, es ist etwas weiter geöffnet, dagegen ist der innere Winkel des linken Auges stärker >ausgezogen`, nur dort ist auch die Karunkel mittels einer Kerbe angegeben. Das linke Unterlid ist konturiert. Das flach anliegende Haar rund um das Gesicht und an der linken Seite, der Ansichtsseite, ist besonders sorgfältig herausgemeißelt, stellenweise in scharf durchgezogenen Linien, die Metallbearbeitung nachahmen. Auch die Ausarbeitung der Lockenzungen an der Oberseite ist von guter Qualität. Hinter dem rechten Ohr und an der Rückseite des Kopfes ist das Haar in Form von dickeren Lockenbüscheln gebildet, doch sind diese Büschel auch an der Rückseite vielfach unterteilt.

An dem ovalen Gesicht ${ }^{193}$ ist der Großteil der Stirn vorgewölbt, unter der horizontalen Linie, die durch das Mittelmotiv des Stirnhaars vorgegeben ist. Die Jochbeine und die Einsenkungen zu Seiten der Nasenflügel sind modelliert, das Philtrum ist nur schwach angegeben. Die mandelförmigen Augen sind von schweren Oberlidern bedeckt, die ihrerseits außen von der Orbitalfalte überlagert werden; die Oberlider überschneiden die Unterlider am äußeren Augenwinkel. Die schweren Unterlider sind von den Wangen abgesetzt, doch ist der Übergang gleitend gestaltet. Beide Brauen überspannen die Augen in einem kontinuierlich ansteigenden, weiten Bogen. Die Brauen sind als Grate gebildet, der untere Rand der Oberlider ist als Kante gestaltet. An den Augen ist die Iris durch einen geritzten Dreiviertelkreis, die Pupille durch eine seichte, bohnenförmige

${ }^{187}$ Ich danke dem damaligen Grabungsleiter F. Krinzinger und der Schnittleiterin U. Quatember für die Überlassung der Funde zur Publikation.

188 Zum Doryphoros in jüngerer Zeit (Auswahl): Kreikenbom 1990, 59-94; Beck - Bol - Bückling 1990 mit mehreren Beiträgen; Beck - Bol 1993 und Moon 1995 mit einer Reihe von Beiträgen; Steuben 1995; Borbein 1996, 71-81; Borbein 2002, 356-359; Bol 2004, 127-129. Folgende Aufsätze zum Doryphoros wurden mir erst im Fahnenstadium des Artikels bekannt: M. Papini, Il Doriforo di Policleto: »Nur künstliche Gedanken«?, in: N. Kreutz - B. Schweizer, Tekmeria. Beiträge für Werner Gauer (Münster 2006) 245-261; J. Lenaghan, On the Use of Roman Copies. Two new Examples of the Doryphoros and Westmacott Ephebe, Eidola 4, 2007, 147-172. Zur Deutung des Doryphoros vgl. Hinweise und Lit. in Franciosi 2003, 51-52 und Cesare 2005, 721-723. Zu einer neuen Rekonstruktion des Typus und einer neuen Identifikation des Doryphoros des Polyklet jetzt Franciosi 2003, Franciosi 2003/2004 und Franciosi 2004, mit Lit.; Franciosi 2006 (die 2. Auflage von Franciosi 2003) war mir nicht zugänglich. Auf diese neue Theorie wird hier nicht eingegangen, da das Ziel dieses Beitrags die Vorstellung der ephesischen Replik ist. - Zum Kopftypus u. a. Steuben 1973, 11-31; Kreikenbom 1990, 81-108 (mit Kat.); Meyer 1995, 73-75; Hartswick 1995.

189 Vgl. o. Kapitel 2.3 (U. Quatember) und 3.3 (A. Waldner). - Zu den technischen Daten des Kopfes vgl. den Katalog am Ende dieses Abschnitts.

${ }^{190} \mathrm{Zu}$ den Abmessungen einiger Kopfrepliken Kalkmann 1893, 17-18; anhand der Apolloniosherme in Neapel, Steuben 1973, 14-15. 26. Zur Kopf- und Gesichtshöhe Berger 1990, 162 f. Zu den einzelnen Repliken vgl. auch die Maßangaben im Katalog von Kreikenbom 1990. - Auf das Maßsystem und den Kanon kann hier nicht eingegangen werden.

191 Steuben 1973, 11-27 Taf. 1-7; Kreikenbom 1990, 174 Kat. III 42 Taf. 172-175.

192 Zur Kopfwendung vgl. Kreikenbom 1990, 81; Meyer 1995, 88. 89. Apolloniosherme: Kreikenbom 1990, Kat. III 42 Taf. 172-175; Statue in Minneapolis: Kreikenbom 1990, Kat. III 1 Taf. 104-107 sowie Hallet 1995a und Meyer 1995; Statue in Neapel: Kreikenbom 1990, Kat. III 2 Taf. 108-112. Vgl. die Zusammenstellung bei Berger 1990, $170 \mathrm{f}$.

193 Zum Gesichtsschnitt Steuben 1973, 14-16; Kreikenbom 1990, 85; Meyer 1995, 74. 


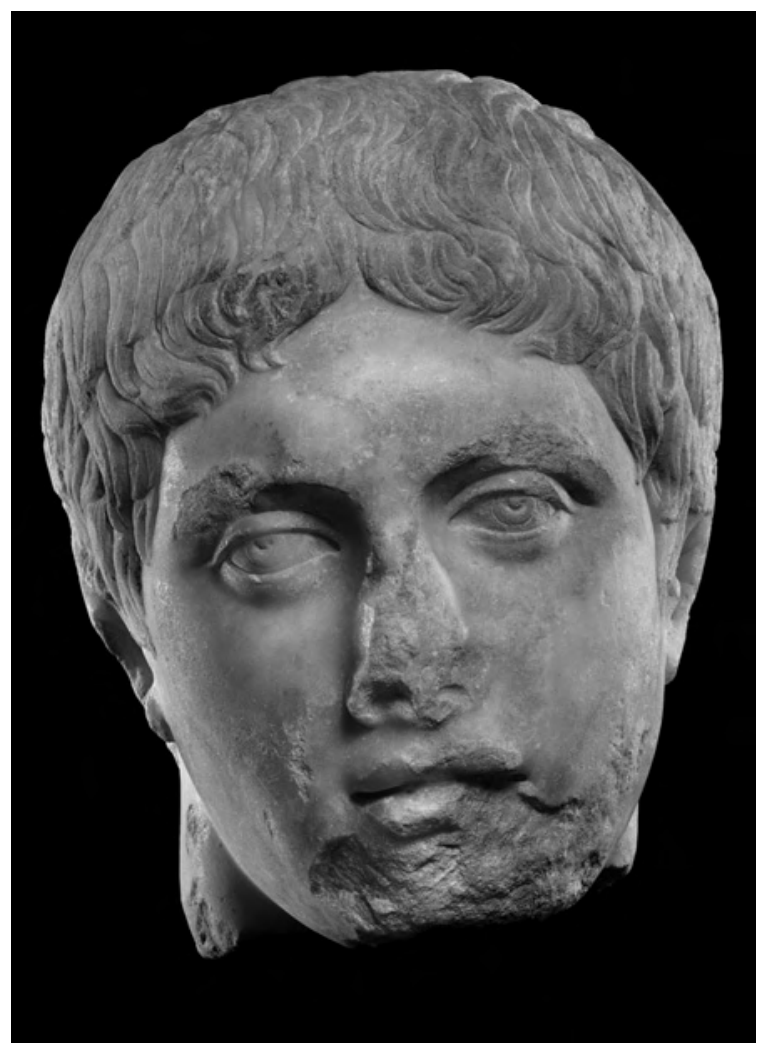

26 Nymphaeum Traiani, Replik des Doryphoros, Vorderseite

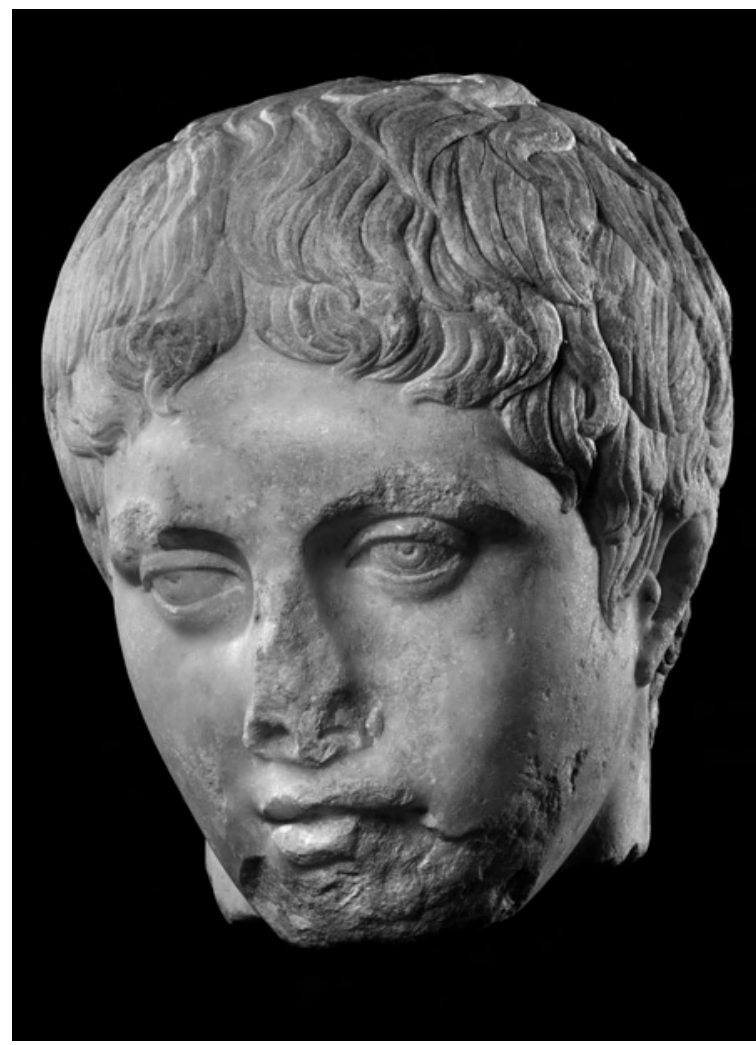

27 Replik des Doryphoros, Hauptansicht

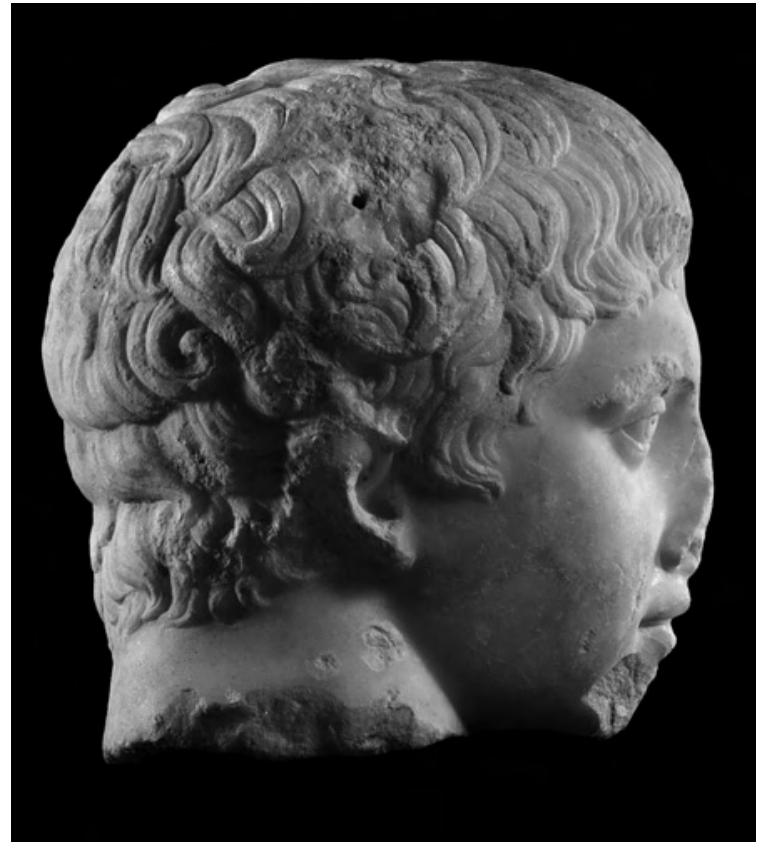

28 Replik des Doryphoros, rechte Kopfseite

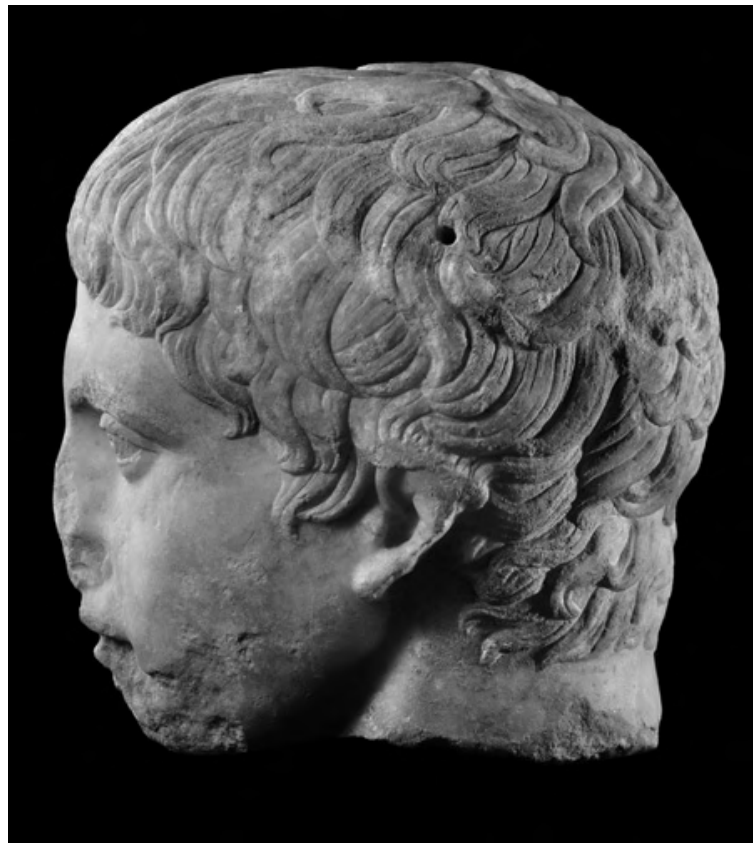

29 Replik des Doryphoros, linke Kopfseite 


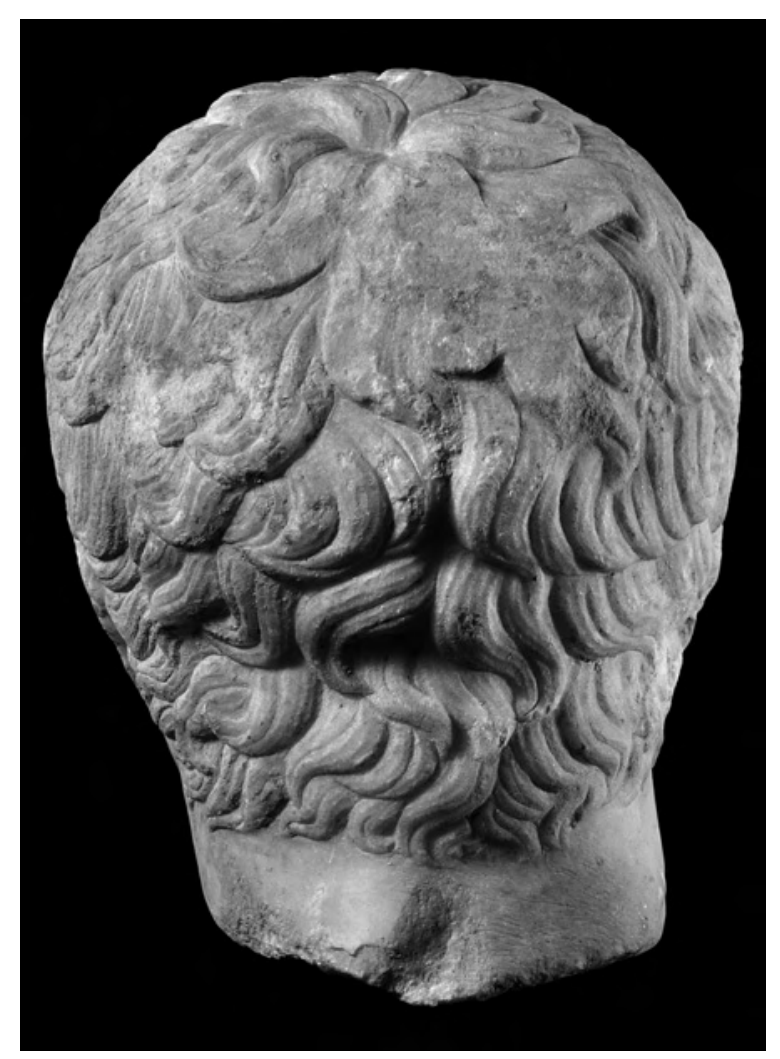

30 Replik des Doryphoros, Rückseite

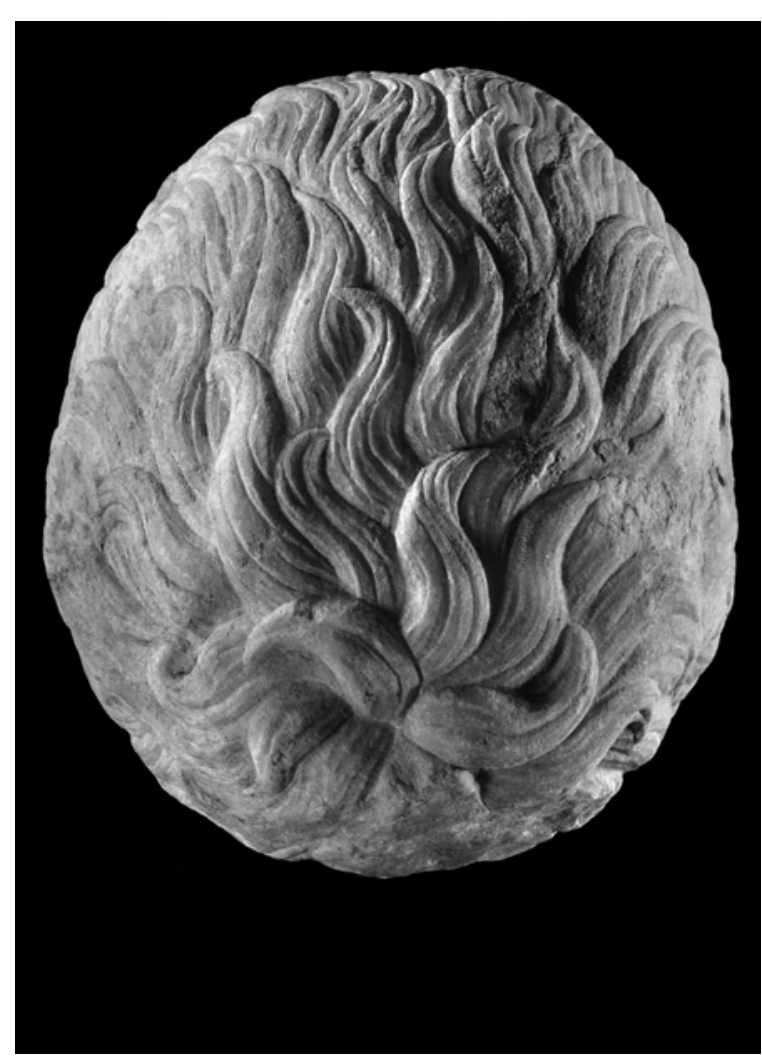

31 Replik des Doryphoros, Oberseite

Kerbung am Rand des oberen Lids markiert. Die Mundspalte ist unter Aussparung des Mittelteils gebohrt, der erhaltene Teil der Oberlippe ist konturiert. Das Haar ist ausschließlich mit dem Meißel herausgearbeitet.

Was das Haar betrifft, so folgte der Bildhauer vor allem in der Gestaltung des Stirnhaars, der linken Kopfseite und der Oberseite in großen Zügen den Systemen des Vorbildes, während sich an der rechten Seite nur vereinzelte Motive des Originals wiederfinden und die Rückseite weitgehend frei gestaltet ist ${ }^{194}$. Vereinfachungen sind an allen Seiten festzustellen. Bereits das Mittelmotiv des Stirnhaars ${ }^{195}$ ist verändert und vereinfacht: Während der rechte Teil der Zange den großen Lockenschwung mit dem kleinen, in die Stirn gedrehten Löckchen - hier verdoppelt zu zwei Löckchen - bewahrt, gefolgt von der kleinen Gabel, sind die Motive im linken Teil der Zange ızusammengezogen` und schematisiert, wodurch das Motiv der weitgespannten Mittelzange verlorengegangen ist. Zwei Bündel von links- bzw. rechtsläufigen kurzen Locken stoßen hier in einer >gefüllten ২ Zange dicht aufeinander, das in die Stirn eingerollte Zangenende - Pendant zum eingerollten Löckchen über dem rechten Auge - fehlt, und von dem folgenden Zangenmotiv mit dem zur Schläfe weisenden Löckchen ist hier nur die unterste Locke geblieben, die horizontal über die Stirn gelegt ist ${ }^{196}$. Dieser Schematisierung entspricht die Verbreiterung der über dem rechten äußeren Augenwinkel in die Stirn gestrichenen Locken (die hier den unteren Arm der kleinen Gabel darstellen) ${ }^{197}$. Die leicht zur

\footnotetext{
${ }_{194}$ Verglichen wird hier vor allem mit der Bronzeherme des Apollonios in Neapel, dem Kopf der Statue in Minneapolis und dem Marmorkopf in Neapel, vgl. Kreikenbom 1990, 81-83 mit Kat. III 42 Taf. 172-175; Kat. III 1 Taf. 104-107 und Kat. III 43 Taf. 176-177. Zur Statue in Minneapolis s. Meyer 1995 und Hallett 1995a. Zum Haarsystem anhand der Bronzeherme vgl. Steuben 1973, 11-14. 21-25.

195 Vgl. Steuben 1973, 11-14 Schema Abb. 1. 2; Hartswick 1995, 163 Abb. 9, 5.

196 Vgl. Hartswick 1995, 163. 164 Abb. 9, 5 (Type 2). Der ephesische Kopf entspricht diesem >Typus in den in die Stirn gestrichenen Locken Nr. 5 und 6. Eine dem ephesischen Kopf vergleichbare Ausbildung des linken Zangenarms an der knabenhaften Umbildung ehemals im Kunsthandel Neapel, Kreikenbom 1990, Kat. III 8 Taf. 129, außerdem >verkümmert an der Replik in Korinth, Kreikenbom 1990, Kat. III 45 Taf. 180-181.

197 Vgl. Hartswick 1995, 163 Abb. 9, 5, die Locken 2-3.
} 
rechten Seite hin verschobene Gabel im zweiten Register über der Mittelzange orientiert sich am Vorbild. Die Gabelarme werden jedoch zu beiden Seiten von weitgehend gleich ausgerichteten und gleichartig gebildeten Sichellocken begleitet, die somit ein durchgehendes zweites Register von Locken über dem Großteil der Stirn bilden, was dem Vorbild nicht entspricht - hier fallen lange Sichellocken in großem Schwung vom Scheitel in die Stirn, über die nur vereinzelte Locken gelegt sind, und zwei von ihnen bilden die Gabel. Auch die vereinzelten rechtsläufigen Locken am Scheitel des Vorbilds sind am ephesischen Kopf zu einem Block rechtsläufiger Sichellocken verdichtet. Diese verdichtenden Elemente tragen zum Eindruck der kompakten Haarkappe bei, aus der sich in der Vorderansicht nur die eingerollten Löckchen über dem rechten Auge lösen. Die prononciert eingerollten Lockenspitzen, die so charakteristisch für die Apolloniosherme $\operatorname{sind}^{198}$, finden an der ephesischen Herme ein Echo nur in den Schläfenlöckchen, in einigen wenigen Motiven an der linken Kopfseite und im Nackenhaar. Die Schläfenlocken an der linken Kopfseite ${ }^{199}$ folgen, wenn auch vereinfacht, dem Schema, die wenigen Löckchen an der rechten Schläfe sind schlicht abwärts, nur die untersten sind nach vorn in die Wange gestrichen ${ }^{200}$.

An der linke Kopfseite ${ }^{201}$ hat sich der Bildhauer insgesamt am Engsten an dem Lockensystem des Vorbilds orientiert: Vorhanden sind charakteristische Motive wie das >gefüllte` Lockenauge schräg über dem Ohr, die hakenförmige Locke links davon (vom Betrachter aus gesehen), die in die Schläfenlocken greift, die Ausrichtung der Lockenbündel über und rechts vom Lockenauge. Die große, hakenförmige Locke rechts vom Lockenauge, die von oben kommend auf den obersten Rand des Ohrs trifft ${ }^{202}$, ist hier allerdings in zwei Reihen gegensätzlich orientierter Locken aufgelöst. Auch hinsichtlich der Löckchen am Nacken hinter dem Ohr folgte der Bildhauer dem Vorbild.

An der rechten Seite ${ }^{203}$ erinnert dagegen nur die Ausrichtung mancher Locken an das hier stark vereinfachte und verkürzte Schema, so die lange schräge Locke, die sich über dem Ohr einrollt, aber - die Stelle ist beschädigt - möglicherweise geteilt ist und in die andere Richtung eingerollt war; die Strähnen, die sich vor und etwa parallel zu dieser langen Locke bewegen und davor auf den oberen Rand der Ohrmuschel treffen, die nach vorn eingerollten Locken hinter dem Ohr, die in die andere Richtung gebogene Locke rechts neben dem Ansatz der langen, schrägen Locke. Am Übergang zur Rückseite setzen sich die dicken, nach vorn eingerollten Büscheln in zwei Registern fort; in der untersten Reihe ist das Haar in kleine Löckchen aufgelöst, was in dieser Form mit dem Entwurf des Vorbilds nichts gemein hat.

Auch in der im mittleren Bereich symmetrisch aufgebauten Gestaltung der Rückseite ${ }^{204}$ entfernt sich der Bildhauer sehr weit vom Vorbild, geblieben ist nur ein zentrales Motiv im dritten Register von unten, das eine Zange mit tiefer ausgreifendem rechtem Zangenarm zeigt. Darüber sitzt, etwa in derselben Achse und vom Vorbild abweichend, eine Gabel. Die Locken zu Seiten dieser Motive sind jeweils nach außen orientiert. Auch die verkürzten Register unter der Zange entsprechen nicht dem Vorbild.

Die Form der Haarspinne und der Duktus der über dem Scheitel zur Vorderseite hin >züngelnden`, flammenartigen, langen Locken orientieren sich jedoch wieder am Vorbild ${ }^{205}$.

Am ephesischen Kopf ist also das Haarsystem des polykletischen Vorbilds an der Ansichts- und auch an der Oberseite recht getreu übernommen, während das System an den anderen Seiten, darunter auch das Stirnhaarmotiv, stark vereinfacht und verändert is $\mathrm{t}^{206}$. Stilistisch gesehen sind die Haare der Vorder- und

198 Vgl. dazu Kreikenbom 1990, 81-83; Hallett 1995b, 151-156; Meyer 1995, 74.

199 Vgl. die Abbildung von vier Repliken bei Hartswick 1995, 164. Dazu den Kopf der Statue in Minneapolis, Meyer 1995 , 70 Abb. 6. 11 und Kreikenbom 1990, Taf. 107 b.

${ }^{200}$ Im Gegensatz zum Lockenduktus an den beiden Köpfen in Neapel und der Statue in Minneapolis, wo die untersten Löckchen zurückgebogen sind. Für Abbildungen vgl. die in Anm. 194 angegebene Lit.

201 Vgl. Steuben 1973, 21-23; Kreikenbom 1990, 84. Zu den Abb. vgl. hier Anm. 199.

${ }^{202}$ Kreikenbom 1990, 84. Deutlich ausgebildet wieder an den beiden Hermen und der Statue in Minneapolis, vgl. Anm. 194.

${ }^{203}$ Vgl. Steuben 1973, 21-23; Kreikenbom 1990, 84. Vgl. hier die beiden Köpfe in Neapel, Anm. 194. Die rechte Seite des Kopfes der Statue in Minneapolis ist durch einen glimmerhältigen Streifen beeinträchtigt; vgl. Meyer 1995, 69 Abb. 6. 9.

${ }^{204}$ Zum Hinterkopf Steuben 1973, 25. Abbildung der Rückseiten der Bronzeherme und der Statue in Minneapolis: Kreikenbom Taf. 175 b und Meyer 1995, 70 Abb. 6. 11.

${ }^{205}$ Zur Haarspinne Steuben 1973, 23-25 Abb. 7. Zusammenstellung von Abbildungen der Oberseiten mehrerer Repliken: Meyer 1995, 84-85.

${ }^{206}$ Vgl. Kreikenbom 1990, 18-19 zur Terminologie der Kopienkritik; 88-94 zu Wiederholungen und Umbildungen im Gegensatz zur >Kernüberlieferung`; Maderna-Lauter 1990, 329-330. 340-341 zur Terminologie, 340-345 zu Nachbildungen nach Werken Polyklets. Hallett 1995 b zur Kopienkritik anhand von Werken Polyklets. 
der Nebenseiten in Form von breiten, an ihrer Oberseite leicht gerundeten Lockenbündeln wiedergegeben, die in ihrer Binnenzeichnung weniger differenziert, weniger von graphischen Werten bestimmt sind als die frühkaiserzeitlichen Repliken. Die scharf durchgezogenen Linien an der linken Seite wirken hier wie saufgesetzt $\measuredangle$. Die plastische Wiedergabe der Locken steigert sich vom Lockengekringel hinter den Ohren bis zu den kräftigen Büscheln an der fast zur Gänze frei geformten Rückseite. Anhand der Gestaltung der besonders plastischen Lockenbüschel direkt unter der Haarspinne lässt sich ein Hinweis für die Datierung gewinnen: Diese sind von einem Netz feiner Grate überzogen, das sich in ähnlicher Form an Porträts Hadrians, frühen Porträts des Antoninus Pius sowie frühantoninischen Prinzenbildnissen findet (wobei die eingerollten Locken an der Rückseite am besten zu vergleichen sind ${ }^{207}$.

Im Gesicht sind - im Vergleich mit der Herme des Apollonios ${ }^{208}$ - die Proportionen durch die Verkleinerung der Augen und wohl auch des Mundes sowie durch die volleren Wangen dahingehend verändert, dass der Eindruck eines knabenhaften Jünglings entsteht. Diese Tendenz zur Verjüngung polykletischer Athleten ist in der kaiserzeitlichen Rezeption oft festzustellen ${ }^{209}$. Das Gesicht zeigt in manchen Details sanfte Übergänge, wie an den Senken unter den Unterlidern und zu Seiten der Nasenflügel; auch das Philtrum ist nur angedeutet. Dagegen wird in den Brauengraten das klassische Vorbild zitiert, das Oberlid ist kantig abgesetzt, linkes Unterlid und Oberlippe sind konturiert. Der im Ausdruck stimmungsvolle, auch stärker geneigte Kopf entspricht dem Zeitsti ${ }^{210}$. Ausdrucksträger sind hier in erster Linie die schmalen Augen mit ihrem unbestimmten Blick, die von schweren Lidern bedeckt sind. Verwandte Augenbildung findet sich bei den frühen antoninischen Prinzenbildnissen ${ }^{211}$. Auch die Augen der ephesischen Replik des lysippischen Eros mit dem Bogen lassen sich vergleichen ${ }^{212}$. Aufgrund seiner Datierung in frühantoninische Zeit gehört der ephesische Kopf in die späteste Epoche der Rezeptionsgeschichte des Doryphoros ${ }^{213}$. Zwei ähnlich stimmungsvolle Wiederholungen oder umgearbeitete Varianten befinden sich in Salt Lake City und - ein Knabenkopf - im New Yorker Privatbesitz ${ }^{214}$.

Der ephesische Kopf nimmt einen wichtigen Platz ein in der Reihe der Kopfrepliken und Wiederholungen aus dem östlichen Bereich des Mittelmeerraums ${ }^{215}$, ist aber auch von besonderer Bedeutung für das Kopienrepertoire, das wir bis jetzt aus Ephesos kennen. Bisher fanden sich in Ephesos vergleichsweise wenige Zeugnisse für die Auseinandersetzung mit dem polykletischen Erbe. Zu nennen sind hier vor allem der nach dem `Diskophoros` gearbeitete Torso und die Kopfreplik des Herakles aus dem Hafengymnasium, umgebildet zu einer Hermesdarstellung. Letztgenannte ist, im Unterschied zur >weich ‘ gearbeiteten ephesischen Kopfreplik des Doryphoros, in der Wiedergabe der Gesichtsdetails und der Haarlocken durchweg von klassizistischer Härte geprägt, deren Anordnung für die Erzielung eines Hell-Dunkel-Effekts aufgelöst ist ${ }^{216}$. Das Fragment eines erhobenen, angewinkelten Armes aus dem Vediusgymnasium ist mit einer Replik des Diadoumenos zu verbinden ${ }^{217}$. Eine Plinthe mit dem Beinpaar einer Statue im polykletischen Standmotiv -

\footnotetext{
${ }^{207}$ Vgl. z. B. zwei Porträtbüsten des Hadrian im Thermenmuseum und in Neapel sowie ein frühes Porträt des Antoninus Pius im Kapitolinischen Museum, Fittschen - Zanker 1994², Beil. 23 und 32 sowie S. 63 Nr. 59 Taf. 68. Zu dieser 〉Schraffur〈Fittschen Zanker 1994², 63 und Fittschen 1999, 34 Taf. 58-59 zur Knabenbüste des Lucius Verus in Kopenhagen im 1. Knabenporträt.

${ }^{208}$ Vgl. Anm. 191 und 192.

${ }^{209} \mathrm{Zu}$ einer durchgreifenden Umbildung des Doryphoros in einen Epheben vgl. die Statue ehemals im Neapler Kunsthandel, Kreikenbom 1990, Kat. III 8 Taf 128. 129; Maderna-Lauter 1990, 345-346, zur >Verjüngung`polykletischer Werke S. 351. 382-383.

${ }^{210}$ Zanker 1974, 44-45. 118.

${ }^{211}$ Vgl. das 1. Knabenbildnis des Marc Aurel oder den Bildnistypus Kopenhagen NCG 713a, Fittschen 1999, 13-21 und 46-49, beide vor oder um $140 \mathrm{n}$. Chr.

${ }^{212}$ Aurenhammer 1990, 85 f. Nr. 64 Taf. 45. Eine Neupublikation des Kopfes und der zugehörigen Fragmente ist geplant.

${ }^{213}$ Vgl. Kreikenbom 1990, 89-90 zu Wiederholungen des 2. Jhs. n. Chr., zur Rezeptionsgeschichte 93-94.

${ }^{214}$ Kopffragment in Salt Lake City und Knabenkopf im New Yorker Privatbesitz, Kreikenbom 1990, Kat. III 60. 65 Taf. 201 b und 207-208; D. Kreikenbom in: Beck - Bol - Bückling 1990, 550-551 Nr. 57. 58; D. Kreikenbom in: Beck - Bol 1993, 337.

${ }^{215}$ Repliken in Korinth, Thera, ehemals in Smyrna, Evangelische Schule, in Damaskus und in Tripolis, Kreikenbom 1990, Kat. III 45 Taf.180. 181 a; III 64 Taf. 205-206; III 52 Taf. 189 a. b; III 7 Taf. 123-127; III 6 Taf. 122.

${ }^{216}$ Aurenhammer 1990, Nr. 159 Taf. 122 a. b; 123 sowie Nr. 7 Taf. 6-7; Kreikenbom 1990, Kat. I 13 Taf. 26 b-27; Kat. IV 13 Taf. 233 b-234; D. Kreikenbom in: Beck - Bol - Bückling 1990, 552 f. Nr. 62.

217 J. Auinger wird dieses Fragment in einem 2009 erscheinenden Supplementband der JRA publizieren (Akten des Convegno $\Vdash L a$ Scultura Romana in Asia Minore«, Mai 2007 in Lecce).
} 


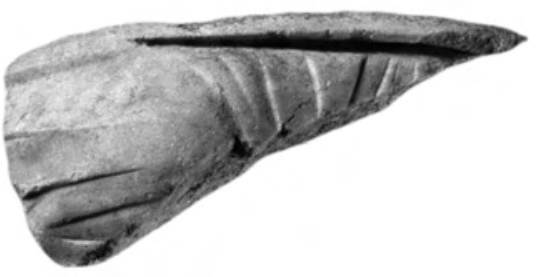

32

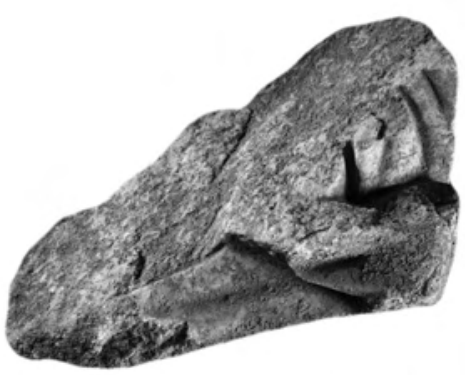

33 Nymphaeum Traiani, Büstenfragment

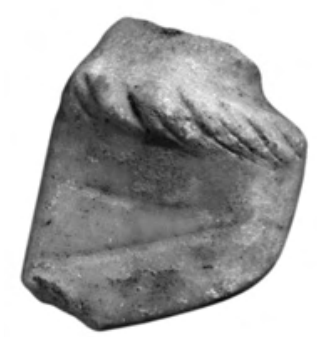

34 Nymphaeum Traiani, Relieffragment: Pferdehuf

mit schräg gestelltem rechten Bein - gehörte zur Ausstattung des flavischen Nymphäums des C. Laecanius Bassus $^{218}$.

In das Haar hoch über den Ohren der ephesischen Replik sind zwei Stiftlöcher für die Anbringung eines Attributs (eines Kranzes?) gebohrt. Stiftlöcher finden sich auch im Haar der Statue aus Leptis Magna (dort im Haar über der rechten Stirnhälfte) ${ }^{219}$. Für die Anstiftung von Flügeln sitzen die Löcher am ephesischen Kopf zu weit außen ${ }^{220}$. Einen Hinweis auf die Deutung gibt aber der dreifach gekerbte, d. h. gebrochene Knorpel der linken Ohrmuschel - das rechte Ohr kann dafür nicht herangezogen werden, da es zur Hälfte abgebrochen ist. Das Pankratiastenohr ist auch an anderen Repliken belegt, an diesen aber zumeist am rechten Ohr deutlich ausgebildet ${ }^{221}$. Ausgehend von diesem Detail schlug in jüngerer Zeit P. C. Bol eine Deutung des Doryphoros als Dioskur Polydeukes, des mythischen Faustkämpfers, vor ${ }^{222}$. Die mythologischen Interpretationen des Doryphoros umfassen mittlerweile - neben Achill - eine Reihe von Helden ${ }^{223}$, in letzter Zeit auch Theseus, anhand einer Statuengruppe im Gymnasium von Messene, zu der ein Torso im Typus gehörte $^{224}$. In jüngster Zeit hat schließlich V. Franciosi, ausgehend von der literarischen Überlieferung und von Beobachtungen an der Statue in Neapel, den Statuentypus ganz von dem überlieferten >Doryphoros des Polyklet getrennt und vorgeschlagen, in dem Typus vielmehr den bei Plinius erwähnten nudus telo incessens zu erkennen 225 .

Zusammen mit der ephesischen Kopfreplik des Doryphoros wurde eine Reihe kleiner Gliedmaßenfragmente (S 2-6) gefunden, deren Zugehörigkeit jedoch nicht nachgewiesen werden kann.

Aus dem Grabungsareal stammen zwei Büstenfragmente. Das größere (S 7 Abb. 32) wurde in SE 30 gefunden, als Teil der Ende des 6./Anfang des 7. Jahrhunderts aufgebrachten Aufschüttungen hinter der Brunnenrückwand ${ }^{226}$. Dieses Fragment einer großen, bekleideten Büste stellt den Nackenteil und den Ansatz der rechten Schulter dar (in der Abb. 32 in einer Ansicht von oben). Bei dem eng anliegenden Gewand handelt es sich offensichtlich um das Untergewand. An der Schulter sind horizontale bzw. leicht schräg verlaufende, flache Falten mit feinen Faltengraten angegeben. An der Nackenpartie breitet sich ein gleichsam symmetrisches System schräger Falten aus. Der Büstenrand dahinter - als Übergang zur konvex gewölbten Rückseite -

\footnotetext{
${ }^{218}$ Aurenhammer 1990, Nr. 156 Taf. 120 b. E. Rathmayr wird die Ausstattung dieses Brunnens in der Publikation M. Aurenhammer K. Jung (Hrsg.), Das Nymphaeum des C. Laecanius Bassus in Ephesos, FiE (in Vorbereitung) vorlegen.

${ }^{219}$ Kreikenbom 1990, Kat. III 6 Taf. 122; Hartswick 1995, 166 Abb. 9. 11.

${ }^{220}$ Vgl. die Flügeln an den Repliken des Hermestypus, Kreikenbom 1990, Kat. II 10 (Einlassungen antik?); II 12. 15.

${ }^{221}$ Hartswick 1995, 165; Bol 2001, 163-169.

${ }^{222}$ Bol 2001, 168-169.

${ }^{223}$ Vgl. Franciosi 2003, 51-52 mit Lit. und die Rezension zu diesem Werk, Cesare 2005, 721-723 mit ausführlicher Lit. zu dieser Frage.

224 Themelis 2001.

${ }^{225}$ Franciosi 2003; Franciosi 2003/2004; Franciosi 2004; Franciosi 2006. Vgl. die Rezension zur 1. Auflage: Cesare 2003, 720-723; vgl. o. Anm. 188.

${ }^{226}$ s. o. Kapitel 2.3 (U. Quatember) und 3.3 (A. Waldner).
} 


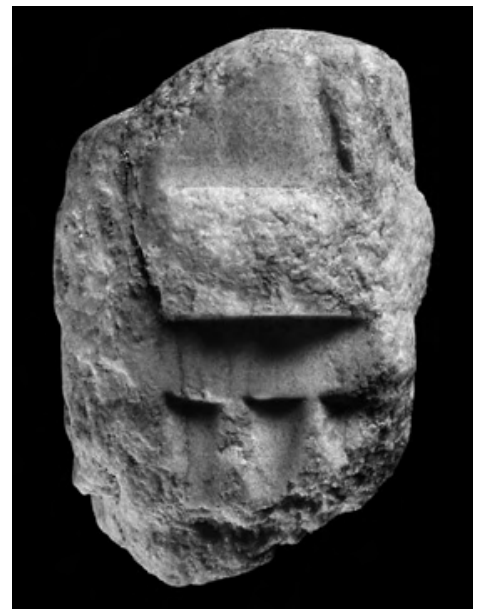

35 Nymphaeum Traiani, Tischfuß, Vorderseite

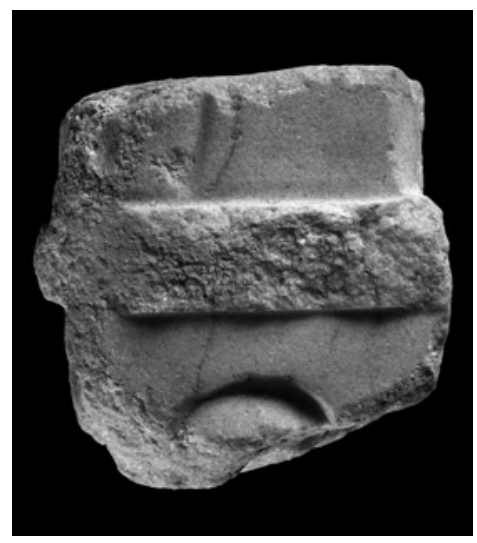

37 Tischfuß, Nebenseite

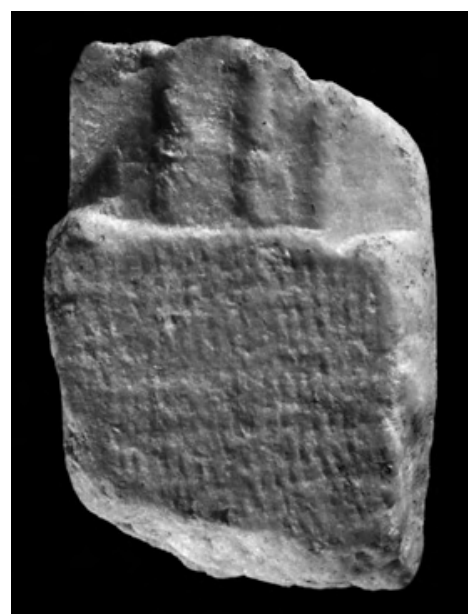

36 Tischfuß, Rückseite

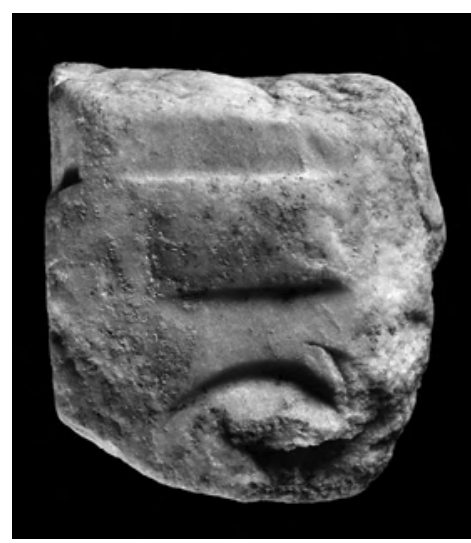

38 Tischfuß, Nebenseite

ist tief unterschnitten. Denkbar ist, angesichts der Größe der Büste und der flachen, durch feine Grate und Ritzungen abgegrenzten Falten, eine spätkaiserzeitliche bis spätantike Datierung.

Das kleinere Büstenfragment S 8 (Abb. 33) stammt aus der Verfüllung (SE 34), einer östlich des Wasserbeckens ausgerissenen Rohrleitung, welche zu einer sekundären Öffnung in der Rückwand der Brunnenfassade im Bereich des östlichen Flügels führte $^{227}$. Der Fundkontext weist in das ausgehende 5 . oder in die erste Hälfte des 6. Jahrhunderts. Bei dem Objekt handelt es sich um ein seitliches Randfragment einer Gewandbüste (ein Rest der Kante hat sich erhalten) - möglicherweise vom linken Rand, wenngleich die Interpretation des Objekts angesichts seines Zustands und der nur schwach konvexen Rundung an der Rückseite schwierig ist. Vielleicht haben wir den Rest einer jener mittel- bis spätkaiserzeitlichen weiblichen Büsten vor uns, an denen der Mantel vor oder unter der Brust verschlungen ist $^{228}$.

Aus der Kalklöschgrube und ihren Nutzungshorizonten (SE 16) stammen ein kleiner Pferdehuf (S 9)

und ein Lockenfragment (S 10); der Fundkontext weist hier an das Ende des 4. oder den Beginn des 5. Jahrhunderts $^{229}$. Das nur $4 \mathrm{~cm}$ hohe, aber nichtsdestoweniger auffallende Fragment mit dem Pferdehuf (S 9 Abb. 34) war offenbar Teil eines Hochreliefs: Es ist rundum ausgearbeitet (die Tiefe beträgt am Huf selbst nur 1,6 cm), abgesehen von einem abgebrochenen Ansatz im oberen Teil der Rückseite, der auf die Verbindung des Fragments mit einem weiteren Reliefteil oder dem Reliefhintergrund weist. Der Huf ist am Übergang zum Pferdebein abgeschlagen, über seinen Ansatz fallen Haarbüschel. Zwei seichte Kerben sind an der Vorderseite des Hufes, der wohl zu dem angehobenen Vorderbein eines nach links bewegten Pferdes gehörte, eingetieft. Auch hier käme eventuell auch eine spätantike Datierung in Frage, wenn man entsprechende Reliefs oder die filigranen Darstellungen auf Elfenbein-Diptychen betrachtet ${ }^{230}$.

Das kleine Lockenfragment eines lebensgroßen, kaiserzeitlichen Kopfes (S 10) zeigt zwei Reihen breiter, nur mit dem Meißel bearbeiteten Sichellocken übereinander, wobei die Ausrichtung der Reihen divergiert.

Das Fragment eines im Querschnitt rechteckigen Tischbeins (S 11) schließlich wurde ebenfalls im Bereich des Nutzungshorizontes der Kalklöschgrube (SE 20) gefunden, in einem Fundkontext des 4. Jahrhun-

\footnotetext{
227 s. o. Kapitel 2.3 (U. Quatember) und 3.3 (A. Waldner).

228 Vgl. z. B. Smith 2006, 243-244 Nr. 128-129 Taf. 100 (J. Lenaghan).

229 s. o. Kapitel 2.2 (U. Quatember) und 3.2 (A. Waldner).

${ }^{230}$ Vgl. z. B. ein Relief in Thessaloniki, Simon 2006, 75 Abb. 46, oder das Wagenrennen auf dem Diptychon der Lampadii in Brescia, Ensoli - La Rocca 2000, Abb. auf S. 121. 123-125 und S. 445-447 Kat. 33.
} 
derts (Abb. 35-38) ${ }^{231}$. Das Fragment ist oben und unten abgeschlagen, zwei der Seitenkanten sowie die vorspringende Leiste an der Vorder- und den Nebenseiten sind großteils weggebrochen. Da alle Seiten dekoriert sind, handelt es sich bei dem Fragment um den Teil eines vierbeinigen Tisches ${ }^{232}$. Die Seite mit dem Ansatz der drei Kanneluren unter der Leiste ist die Vorderseite (Abb. 35). Der Großteil der Rückseite ist als rechteckiges, mit dem Zahneisen bearbeitetes Feld ausgeführt, darüber sind drei vertikale Leisten in flachem Relief angegeben (Abb. 36). Die etwas längeren Nebenseiten weisen unter der Leiste den Rest eines gekerbten kreisförmigen Ornaments auf (Abb. 36. 37). Erhalten hat sich jener Teil des Tischbeins, der den Übergang vom Schaft zum Ansatz der Felidenpranke markiert; dieser wird oft durch eine vorspringende Leiste betont $^{233}$. Dieses Detail zeigen auch die Tischbeine des massiven Tisches aus dem Peristylhof der Wohneinheit 7 des Hanghauses 2 in Ephesos, die Vorderseite der Schäfte ist hier mit Kanneluren verziert ${ }^{234}$. Die Vergleichsbeispiele für das Tischbeinfragment weisen in die Kaiserzeit. Vierbeinige Tische sind in Ephesos durch den späthellenistischen Tisch mit vegetabilem Dekor aus dem späthellenistisch-frühkaiserzeitlichen Peristylhaus unter der Domus des Hanghauses 1 belegt $^{235}$.

\section{Katalog der Marmorfunde (Skulptur und Mobiliar)}

\section{S 1}

Kopfreplik des Doryphoros

FO: SE 10, Aufschüttung über dem Ausriss der Bleileitung

AO: Selçuk, Efes Müzesi 23/4/05

Grobkörniger, weißer Marmor mit grauer Äderung (eine dunkle Ader verläuft z. B. von der r. Schläfe zur Wange), gelblich braune Patina; Wurzelfasern. Die bei der Auffindung stark versinterte Oberfläche wurde mittels Laser freigelegt.

H26,8 cm;HKinnboden-Scheitel26,4 cm;Hdes Gesichts $19 \mathrm{~cm}$ (Kinnbruch - Zangenmitte des Stirnhaars); B max. 21,7 cm; T max. 25,5 cm (Stirn - Hinterkopf); B der Schläfen 14,7 cm; Abstand der inneren Augenwinkel 3,5 cm; Abstand der äußeren Augenwinkel 10,1 cm; Abstand der Lider außen $11 \mathrm{~cm}$; B des Mundes 5,1 cm; L der Nase $7 \mathrm{~cm}$; Abstand der Stiftlöcher im Haar 19,7 cm; Stiftloch r.: T 2,2 cm, Dm 0,5 cm; Stiftloch 1.: T 2,7 cm, Dm 0,7 cm.

In Kinnhöhe etwa horizontal abgeschlagen. Mittellinie des Gesichts beschädigt: abgebrochen Großteil der Nase und des Mundes, Kinnspitze, Oberfläche des Kinns und der 1. Wange. Bestoßungen an Brauen, 1. Lid, Wangen, Hals, Rändern der Ohren. Haar stellenweise bestoßen, bes. um das Stiftloch an der $r$. Seite und am hinteren Teil des Wirbels.

Inkarnat gut geglättet. Augenzeichnung: Iris als feiner Dreiviertelkreis, Pupille durch seichte, bohnenförmige Kerbe markiert. Im 1. inneren Augenwinkel Karunkel angegeben. L. Unterlid und Oberlippe konturiert. Mundspalte gebohrt. Im Haar nur Meißelarbeit; an der 1. Kopfseite Locken stellenweise durch scharfe Rillen unterteilt. Je ein Stiftloch sitzt etwa $8 \mathrm{~cm}$ über den Ohren im Haar.

Dat. des Fundkontexts: Ende 6./Anfang 7. Jh. n. Chr.

Dat.: frühantoninisch
S 2-6

Fragmente von Gliedmaßen

FO: wie $\mathrm{S} 1$

AO: Depot des österreichischen Grabungshauses Grobkörniger hellgrauer Marmor, Mörtelreste, Wurzelfasern

Alle Fragmente sind abgesehen von einer bearbeiteten Fläche an allen Seiten gebrochen.

S 2: L $11,5 \mathrm{~cm} ; \mathrm{S} 3: \mathrm{L} 7,5 \mathrm{~cm} ; \mathrm{S} 4: \mathrm{L} 4,5 \mathrm{~cm}$

$\mathrm{S} 3$ und 4 könnten aufgrund von Marmor und Erhaltungszustand zusammengehören.

S 5: L 7,5 cm; $\mathrm{S}$ 6: L $5 \mathrm{~cm}$

Dat. des Fundkontexts: frühantoninisch

\section{S 7}

Abb. 32

Fragment einer Gewandbüste

FO: SE 30, Aufschüttung

AO: Depot des österreichischen Grabungshauses

Grobkörniger, hellgrauer Marmor, vor allem an der Rückseite starke Mörtelreste; Wurzelfasern

H $12 \mathrm{~cm}$ (?) (die H hängt von der Position des Fragments ab); B 30,3 cm; B des Büstenrandes 2,5-3 cm

Erhalten Nackenpartie und Ansatz der r. Schulter. Gewandfalten und Büstenrand bestoßen.

Faltenpartien geglättet, Büstenrand und Rückseite aufgeraut. Büstenkante am Übergang zur Nackenpartie unterschnitten.

Dat. des Fundkontexts: Ende 6./Anfang 7. Jh. n. Chr.

Dat.: späte Kaiserzeit oder spätantik

\section{S 8}

Abb. 33

Fragment einer Gewandbüste

FO: SE 34, Rohrgrube

\footnotetext{
${ }^{231}$ s. o. Kapitel 2.2 (U. Quatember) und 3.2 (A. Waldner).

${ }^{232} \mathrm{Zu}$ vierbeinigen Tischen vgl. Moss 1988, 44-50; Carolis 2007, 93-95. 111. 163-165 A 1 Taf. 6.

${ }^{233}$ Moss 1988, 48.

${ }^{234}$ Vetters 1982, 74 Taf. 10. 11. Diesen Hinweis verdanke ich U. Quatember, die das Marmorinventar aus den Hanghäusern in Ephesos bearbeitet, vgl. Quatember 2003, 2005b und Quatember (in Druckvorbereitung) b.

${ }^{235}$ Lang-Auinger 2003, 122-124. 135-136 M1 Taf. 52-54. 159-161.
} 
AO: Depot des österreichischen Grabungshauses Grobkörniger, hellgrauer Marmor, dicht von grauen Ablagerungen überzogen

H $15 \mathrm{~cm}$; B 23,5 cm; T max. 7,5 cm

Erhalten offensichtlich Fragment des 1. Büstenteils (?) mit Rest der 1. Kante. Oben, unten und an r. Seite (?) gebrochen. Falten beschädigt. Zahneisen an der Rückenseite und am Büstenrand.

Dat. des Fundkontexts: Ende 5./Anfang 6. Jh. n. Chr.

Dat.: mittlere bis spätere Kaiserzeit

\section{S 9}

Abb. 34

Kleinformatiger Pferdehuf, Relieffragment FO: SE 16, Kalklöschgrube und Nutzungshorizonte AO: Depot des österreichischen Grabungshauses Mittelkörniger, weißer Marmor, Wurzelfasern H $4 \mathrm{~cm}$; B max. 3,5 cm; T max. 2,05 cm inkl. Ansatz $(1,6 \mathrm{~cm}$ direkt am Huf)

Erhalten hat sich scheibenförmiger Pferdehuf von geringer Tiefe; oben am Ansatz des Beins abgeschlagen. Spitze des Hufs abgebrochen. An Rückseite abgebrochener Ansatz (Verbindung zu Reliefgrund oder weiterem Teil der Figur?). Oberfläche geglättet, auch an Rückseite gut ausgearbeitet.

Dat. des Fundkontexts: Ende 4. Jh. n. Chr.

Dat.: kaiserzeitlich oder spätantik?

\section{S 10}

Lockenfragment von lebensgroßem Kopf

FO: SE 16, Kalklöschgrube und Nutzungshorizonte

AO: Depot des österreichischen Grabungshauses

Grobkörniger, hellgrauer Marmor, Mörtelreste, Wurzelfasern

L $9,5 \mathrm{~cm} ; \mathrm{B} 5,5 \mathrm{~cm} ; \mathrm{T} 5,3 \mathrm{~cm}$

Rund um die erhaltene ausgearbeitete Fläche abgebrochen bis auf ein Stück einer Fläche, die evemtuell als Anstückungsfläche geglättet wurde. Locken mit dem Meißel herausgearbeitet. Dat. des Fundkontexts: Ende 4. Jh. n. Chr. Dat.: kaiserzeitlich

\section{S 11}

Abb. 35-38

Fragment eines Tischbeins

FO: SE 20, Nutzungshorizont der Kalklöschgrube

AO: Depot des österreichischen Grabungshauses

Grobkörniger, hellgrauer Marmor, Wurzelfasern; bei der Auffindung von Mörtelpackung bedeckt

H 14,4 cm; B 10,5 und 9 bzw. 9,4 cm (an der Leiste und am Schaft gemessen); T 13,6 bzw. 12,4 cm (an Leiste und Schaft)

Erhalten hat sich der Teil am Übergang von Schaft zu Felidenpranke. Oben und unten abgeschlagen. Beschädigt sind zwei Längskanten sowie die vorspringende Leiste an Vorder- und Nebenseiten. Bestoßungen, Sprünge im Stein. Alle Seiten geglättet bis auf unteren Teil der Rückseite (Zahneisen).

Dat. des Fundkontexts: 3./4. Jh. n. Chr.

Dat.: kaiserzeitlich

M. Aurenhammer

\section{Zusammenfassung}

Die Befunde im Bereich der Strukturen nordöstlich des Nymphaeum Traiani lassen sich im Wesentlichen in drei Perioden untergliedern: Die ergrabenen kaiserzeitlichen Strukturen entziehen sich aufgrund der kleinen Grabungsfläche einer eindeutigen Interpretation; es könnte sich jedoch um ein - möglicherweise zu einem Wohnbau gehörendes - Hofareal gehandelt haben. Die zur Wasserführung des Nymphaeum Traiani gehörenden Tonleitungen wurden offenbar in severischer Zeit einer Reparatur unterzogen. Im 5./6. Jahrhundert wurden im Areal handwerkliche Tätigkeiten durchgeführt, wobei man in der Südwestecke in einer Grube Kalk löschte bzw. seinsumpfteく. Im 6./7. Jahrhundert wurden mehrere Aufschüttungen eingebracht, darunter ein Stratum, das auffallend viele Marmorfragmente beinhaltete, unter ihnen den Kopf einer Replik des Doryphoros. Die letzte Bauaktivität in diesem Areal lässt sich mit hoher Wahrscheinlichkeit auf die erste Hälfte des 7. Jahrhunderts $\mathrm{n}$. Chr. eingrenzen; dies stimmt mit anderen bislang von der Kuretenstraße bekannten Befunden überein ${ }^{236}$.

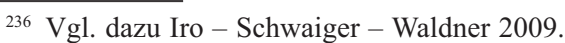




\section{Abkürzungsverzeichnis}

Zusätzlich zu den vom Österreichischen Archäologischen Institut <http://www.oeai.at/publik/autoren.html> empfohlenen Abkürzungen werden folgende verwendet:

$\begin{array}{llll}\text { AO } & \text { Aufbewahrungsort } & \text { Mü } & \text { Münze } \\ \text { BDm } & \text { Bodendurchmesser } & \mathrm{NT} & \text { Nymphaeum Traiani } \\ \text { Bfrgt. } & \text { Bodenfragment } & \mathrm{R} & \text { Rand } \\ \text { erh. } & \text { erhalten } & \text { RDm } & \text { Randdurchmesser } \\ \text { FB } & \text { Farbe Bruch } & \text { Rfrgt. } & \text { Randfragment } \\ \text { Fa } & \text { Farbe außen } & \text { S } & \text { Skulptur } \\ \text { Fi } & \text { Farbe innen } & \text { SE } & \text { Stratigraphische Einheit } \\ \text { FK } & \text { Fundkomplex } & \text { Sek. Ofl. } & \text { sekundäre Oberfläche } \\ \text { Hfrgt. } & \text { Henkelfragment } & \text { Wfrgt. } & \text { Wandfragment } \\ \text { M } & \text { Mauer } & & \end{array}$

\section{Abgekürzt zitierte Literatur}

Auinger 2009

J. Auinger, Zum Umgang mit Statuen hoher Würdenträger in spätantiker und nachantiker Zeit entlang der Kuretenstraße in Ephesos. Fundorte und Fundumstände, in: Ladstätter 2009 (in Druck).

Aurenhammer 1990 M. Aurenhammer, Die Skulpturen von Ephesos. Idealplastik I, FiE 10, 1 (Wien 1990).

Aurenhammer (in Druckvorbereitung) M. Aurenhammer (Hrsg.), Die Skulpturen von Ephesos. Bildwerke aus Stein und Bronze. Die Porträts I, FiE 10, 3 (in Druckvorbereitung).

Bammer 1972-1975 A. Bammer, Grabungen in Ephesos von 1960-1969 bzw. 1970. Architektur, ÖJh 50, 1972-1975, Beibl. 381-406.

Bammer - Fleischer - Knibbe 1974 A. Bammer - R. Fleischer - D. Knibbe, Führer durch das Archäologische Museum in Selçuk - Ephesos (Wien 1974).

Bass - van Doorninck 1971 G. F. Bass - F. H. van Doorninck Jr., A Fourth-Century Shipwreck at Yassi Ada, AJA 75, 1971, $27-37$.

Bass - van Doorninck 1982 G. F. Bass - F. H. van Doorninck Jr., Yassi Ada. A Seventh Century Byzantine Shipwreck, The Nautical Archaeology Series 1 (Texas 1982).

Beck - Bol $1993 \quad$ H. Beck - P. C. Bol (Hrsg.), Polykletforschungen (Berlin 1993).

Beck - Bol - Bückling 1990 H. Beck - P. C. Bol - M. Bückling, Polyklet. Ausstellung Frankfurt am Main (Mainz 1990).

Berger 1990 E. Berger, Zum Kanon des Polyklet, in: Beck - Bol - Bückling 1990, 156-184.

Beyll 1993 D. Beyll, Terra Sigillata aus der Marienkirche in Ephesos, BerMatÖAI 5 (Wien 1993) 5-45.

Bezeczky 2005 T. Bezeczky, Late Roman Amphorae from the Tetragonos-Agora in Ephesus, in: F. Krinzinger (Hrsg.), Spätantike und mittelalterliche Keramik aus Ephesos, DenkschrWien $332=$ AForsch 13 (Wien 2005) 203-229.

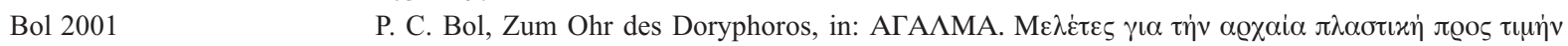

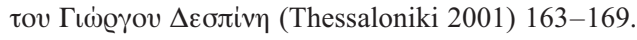

Bol 2004 P. C. Bol, Polyklet, in: P. C. Bol (Hrsg.), Die Geschichte der Bildhauerkunst II. Klassische Plastik (Mainz 2004) 123-132.

Bonifay 2004 M. Bonifay, Études sur la céramique romaine tardive d'Afrique, BAR IntSer 1301 (Oxford 2004).

Bonifay - Piéri 1995 M. Bonifay - D. Piéri, Amphores du Ve au VIIe s. à Marseille: nouvelles données sur la typologie et le contenu, JRA 8, 1995, 94-120.

Borbein 1996 A. H. Borbein, Polykleitos, in: O. Palagia - J. J. Pollitt (Hrsg.), Personal Styles in Greek Sculpture, Yale Classical Studies 30 (Cambridge 1996) 66-90.

Borbein 2002 A. H. Borbein, Polyklet, in: Die griechische Klassik. Idee oder Wirklichkeit. Ausstellung und Katalog Berlin/Bonn (Mainz 2002) 354-363.

Carolis 2007

E. de Carolis, Il mobile a Pompei ed Ercolano, Studia Archeologica 151 (Rom 2007).

Cesare 2005

R. di Cesare, Rezension zu Franciosi 2003, ASAtene 8, 2005, 720-723.

Cottica 2000

D. Cottica, Late Roman Imported and Locally produced Pottery from Hierapolis (Pamukkale, Turkey): Preliminary Evidence, ReiCretActa 36, 2000, 49-56.

Czurda-Ruth 2007

B. Czurda-Ruth, Hanghaus 1 in Ephesos. Die Gläser, FiE 8, 7 (Wien 2007).

Dix 1982

B. Dix, The Manufacture of Lime and Its Uses in the Western Roman Provinces, OxfJArch 1, 1982, $331-345$. 
Ensoli - La Rocca 2000

Fittschen 1999

Fittschen - Zanker $1994^{2}$

Filgis - Blum - Aslan 2005

Franciosi 2003

Franciosi 2003/2004

Franciosi 2004

Franciosi 2006

Gassner 1997

Groh 2006

Hallett 1995a

Hallett $1995 b$

Harter 1999

Hartswick 1995

Hayes 1968

Hayes 1971

Hayes 1972

Hayes 1985

İnan - Rosenbaum 1966

İnan - Alföldi-Rosenbaum 1979

S. Ensoli - E. La Rocca. Aurea Roma. Dalla città pagana alla città Cristiana. Ausstellungskatalog Rom (Rom 2000).

K. Fittschen, Prinzenbildnisse antoninischer Zeit (Mainz 1999).

K. Fittschen - P. Zanker, Katalog der römischen Porträts in den Capitolinischen Museen und den anderen kommunalen Sammlungen der Stadt Rom I ²(Mainz 1994).

M. N. Filgis - S. W. E. Blum - R. Aslan, Traditionelle Kalkbrennerei in Gökçebayır, StTroica 15, 2005, 221-245.

V. Franciosi, Il >Doriforo< di Policleto (Napoli 2003).

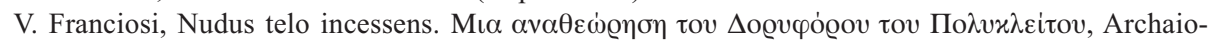
gnosia 12, 2003/2004, 277-295.

V. Franciosi, Il Doriforo di Policleto. Un'indagine filologica a 140 anni dall'identificazione, Eidola 1, 2004, 61-89.

V. Franciosi, Il >Doriforo< di Policleto ${ }^{2}$ (Napoli 2006).

V. Gassner, Das Südtor der Tetragonos-Agora. Keramik und Kleinfunde, FiE 13, 1, 1 (Wien 1997).

S. Groh, Neue Forschungen zur Stadtplanung in Ephesos, ÖJh 75, 2006, 47-116.

C. H. Hallett, The Replica of Polykleitos' Doryphoros in the Minneapolis Institute of Arts: An Archaeological Description, in: Moon 1995, 116-120.

C. H. Hallett, Kopienkritik and the Works of Polykleitos, in: Moon 1995, 121-160.

G. Harter, Römische Gläser des Landesmuseums Mainz (Wiesbaden 1999).

K. J. Hartswick, Head Types of the Doryphoros, in: Moon 1995, 161-176.

J. W. Hayes, A Seventh Century Pottery Group, DOP 22, 1968, 203-216.

J. W. Hayes, A New Type of Early Christian Ampulla, BSA 66, 1971, 243-247.

J. W. Hayes, Late Roman Pottery (London 1972).

J. W. Hayes, Sigillate orientali, in: Atlante delle forme ceramiche II, EAA Suppl. (Rom 1985).

J. İnan - E. Rosenbaum, Roman and Early Byzantine Portrait Sculpture in Asia Minor (London 1966).

Iro - Schwaiger - Waldner 2009 kei. Neue Funde (Mainz 1979).

Isings 1957

Kalkmann 1893

Kreikenbom 1990

Ladstätter 2000

Ladstätter 2003

Ladstätter 2005

Ladstätter 2007

Ladstätter 2008

Ladstätter 2009 (in Druck)

Ladstätter - Sauer 2005

Ladstätter - Steskal 2009

Lang-Auinger 2003

Maderna-Lauter 1990

Meriç 2002

Metaxas 2005

Meyer 1995
D. Iro - H. Schwaiger - A. Waldner, Die Grabungen des Jahres 2005 in der Süd- und Nordhalle der Kuretenstraße. Ausgewählte Befunde und Funde, in: Ladstätter 2009 (in Druck).

C. Isings, Roman Glass from Dated Finds (Groningen 1957).

A. Kalkmann, Proportionen des Gesichts in der griechischen Kunst, BWPr 53 (Berlin 1893).

D. Kreikenbom, Bildwerke nach Polyklet (Berlin 1990).

S. Ladstätter, Ein flavischer Fundkomplex aus dem Hanghaus 2 in Ephesos, ReiCretActa 36, 2000, 97-107.

S. Ladstätter u. a., Ein hellenistischer Brunnen in SR 9C, in: C. Lang-Auinger (Hrsg.), Das Hanghaus 1 in Ephesos. Ausstattung und Funde, FiE 8, 4 (Wien 2003) 22-70.

S. Ladstätter, Keramik, in: H. Thür, Das Hanghaus 2 in Ephesos. Die Wohneinheit 4. Baubefund, Ausstattung, Funde, FiE 8, 6 (Wien 2005) 230-358.

S. Ladstätter, Mode oder politisches Manifest? Überlegungen zur Übernahme römischen Formenguts in der frühkaiserzeitlichen Keramik von Ephesos, in: M. Meyer (Hrsg.), Neue Zeiten - Neue Sitten. $\mathrm{Zu}$ Rezeption und Integration römischen und italischen Kulturguts in Kleinasien, WForsch 12 (Wien 2007) 203-220.

S. Ladstätter, Keramik, in: M. Steskal - M. La Torre (Hrsg.), Das Vediusgymnasium von Ephesos, FiE 14, 1 (Wien 2008) 97-189.

S. Ladstätter (Hrsg.), Neue Forschungen zur Kuretenstraße von Ephesos. Akten des Symposiums für Hilke Thür vom 13. Dezember 2006 an der Österreichischen Akademie der Wissenschaften, DenkschrWien $=$ AForsch 2009 (in Druck).

S. Ladstätter - R. Sauer, Late Roman C-Ware und lokale spätantike Feinware aus Ephesos, in: F. Krinzinger (Hrsg.), Spätantike und mittelalterliche Keramik aus Ephesos, DenkschrWien $332=$ AForsch 13 (Wien 2005) 143-201.

S. Ladstätter - M. Steskal, Die Grabungen 1999 im Bereich der Alytarchenstoa, in: Ladstätter 2009 (in Druck).

C. Lang-Auinger (Hrsg.), Hanghaus 1 in Ephesos. Funde und Ausstattung, FiE 8, 4 (Wien 2003).

C. Maderna-Lauter, Polyklet in Rom, in: Beck - Bol - Bückling 1990, 328-392.

R. Meriç, Späthellenistisch-römische Keramik und Kleinfunde aus einem Schachtbrunnen am Staatsmarkt in Ephesos, FiE 9, 3 (Wien 2002).

S. Metaxas, Frühbyzantinische Ampullen und Amphoriskoi aus Ephesos, in: F. Krinzinger (Hrsg.), Spätantike und mittelalterliche Keramik aus Ephesos, DenkschrWien $332=$ AForsch 13 (Wien 2005) 67-123.

H. Meyer, A Roman Masterpiece, in: Moon 1995, 65-115. 
Miltner 1937

Miltner 1959a

Miltner 1959b

Miltner 1960

Mitsopoulos-Leon 2007

Moon 1995

Moss 1988

Peacock - Williams 1986

Quatember 2003

Quatember 2005a

Quatember 2005b

Quatember 2006

Quatember 2007

schen Kulturguts in Kleinasien, WForsch 12 (Wien 2007) 101-113.

Quatember (in Druckvorbereitung) a U. Quatember, Das Nymphaeum Traiani in Ephesos (in Druckvorbereitung).

Quatember (in Druckvorbereitung) b U. Quatember, Marmorinventar, in: F. Krinzinger (Hrsg.), Das Hanghaus 2 in Ephesos. Wohneinheiten 1 und 2. Baubefund, Ausstattung, Funde, FiE 8, 8 (in Druckvorbereitung).

Reynolds 1998 P. Reynolds, Eastern Mediterranean amphorae between 500 and 700: a view from Italy, in: Ceramica in Italia: VI-VII secolo. Atti del convegno in onore di John W. Hayes, 11-13 maggio 1995 (Rom 1998) $157-184$.

Rogl 2004

Sauer - Ladstätter 2005

Scherrer 1995

Scherrer 2001

Simon 2006

Smith 2006

Steuben 1973

Steuben 1995

Steskal 2008

Themelis 2001

Turnovsky 2005a

Turnovsky 2005b

van Doorninck 1976

Vetters 1982

Wiplinger 2006

F. Miltner, Die Einzelfunde (Lampen), in: Das Cömeterium der Sieben Schläfer, FiE 4, 2 (Wien 1937) 93-200.

F. Miltner, Die österreichischen Ausgrabungen in Ephesos im Jahre 1958, AnzWien 96, 1959, $31-43$.

$315-380$.

F. Miltner, XXIV. Vorläufiger Bericht über die Ausgrabungen in Ephesos, ÖJh 45, 1960, Beibl. $1-76$.

.

. Moon (Hrsg.), Polykleitos, the Doryphoros, and Tradition (Madison 1995).

C. Peacock - D. F. Williams, Amphorae and the Roman Economy (London 1986).

U. Quatember, Zur Grabungstätigkeit F. Miltners an der Kuretenstraße, in: B. Brandt - V. Gassner S. Ladstätter (Hrsg,), Synergia. Festschrift Friedrich Krinzinger I (Wien 2005) 271-278.

U. Quatember, Marmorinventar, in: H. Thür, Das Hanghaus 2 in Ephesos. Die Wohneinheit 4. Baubefund, Ausstattung, Funde, FiE 8, 6 (Wien 2005) 409-413.

U. Quatember, The Water Management and Delivery System of the Nymphaeum Traiani at Ephesus, in: G. Wiplinger (Hrsg.), Cura Aquarum in Ephesus. Proceedings of the Twelfth International Congress on the History of Water Management and Hydraulic Engineering in the Mediterranean Region, Ephesus/Selçuk, Turkey, October 2-10, 2004, BaBesch Suppl. 12 = SoSchrÖAI 42 (Leuven 2006) 73-77.

U. Quatember, Neue Zeiten - Alte Sitten? Ti. Claudius Aristion und seine Bauten in Ephesos, in: M. Meyer (Hrsg.), Neue Zeiten - Neue Sitten. Zu Rezeption und Integration römischen und italiC. Rogl, $\mathrm{Zu}$ dem Produktionsbeginn schwarzer Sigillata in Ephesos. Die Evidenz der Tetragonos Agora, ÖJh 73, 2004, 207-219.

R. Sauer - S. Ladstätter, Mineralogisch-petrographische Analysen von frühbyzantinischen Ampullen und Amphoriskoi aus Ephesos, in: F. Krinzinger (Hrsg.), Spätantike und mittelalterliche Keramik aus Ephesos, DenkschrWien 332 = AForsch 13 (Wien 2005) 125-136.

P. Scherrer (Hrsg.), Ephesos. Der neue Führer (Wien 1995).

P. Scherrer, The Historical Topography of Ephesos, in: D. Parrish (Hrsg.), Urbanism in Western Asia Minor. New studies on Aphrodisias, Ephesos, Hierapolis, Pergamon, Perge and Xanthos, JRA Suppl. 45 (Portsmouth 2001) 57-87.

E. Simon, Pferde in Mythos und Kunst der Antike (Ruhpolding 2006).

R. R. R. Smith, Aphrodisias 2. Roman Portrait Statuary from Aphrodisias (Mainz 2006).

H. v. Steuben, Der Kanon des Polyklet. Doryphoros und Amazone (Tübingen 1973).

H. v. Steuben, Der Doryphoros und der Kanon Polyklets, StädelJb 15, 1995, 7-18.

M. Steskal, Glas, in: M. Steskal - M. La Torre (Hrsg.), Das Vediusgymnasium von Ephesos, FiE 14, 1 (Wien 2008) 189-204.

P. G. Themelis, The Messene Theseus and the Ephebes, in: Zona archaeologica. Festschrift Hans Peter Isler (Bonn 2001) 407-419.

P. Turnovsky, The Morphological Repertory of Late Roman/Early Byzantine Coarse Wares in Ephesos, in: $1^{\text {st }}$ International Conference on Late Roman Coarse Wares, Cooking Wares and Amphorae in the Mediterranean: Archaeology and Archaeometry, Barcelona 14-16 March 2002, BARIntSer 1340 (Oxford 2005) 635-646.

P. Turnovsky, Late Antique and Byzantine Pottery of the Church of St. Mary in Ephesos. An Introduction, ReiCretActa 39, 2005, 217-224.

F. van Doorninck Jr., The $4^{\text {th }}$ Century Wreck at Yassi Ada. An Interim Report on the Hull, IntJNautA 5, 1976, 115-131.

H. Vetters, Ephesos. Vorläufiger Grabungsbericht 1981, AnzWien 119, 1982, 62-101.

G. Wiplinger, Wasser für Ephesos. Stand der Erforschung der Wasserversorgung, in: G. Wiplinger (Hrsg.), Cura Aquarum in Ephesus. Proceedings of the Twelfth International Congress on the History of Water Management and Hydraulic Engineering in the Mediterranean Region, Ephesus/Selçuk, Turkey, October 2-10, 2004, BaBesch Suppl. 12 = SoSchrÖAI 42 (Leuven 2006) 23-37. 
Zabehlicky-Scheffenegger u. a. 1996 S. Zabehlicky-Scheffenegger - R. Sauer - G. Schneider, Graue Platten aus Ephesos und vom Magdalensberg, in: M. Herfort-Koch - U. Mandel - U. Schädler (Hrsg.), Hellenistische und kaiserzeitliche Keramik des östlichen Mittelmeergebietes, Kolloquium Frankfurt 1995 (Frankfurt 1996) 41-59.

Zanker 1974

P. Zanker, Klassizistische Statuen (Mainz 1974).

Mag. Dr. Ursula Quatember

\% Österreichisches Archäologisches Institut, Franz Klein-Gasse 1, A-1190 Wien

E-Mail: ursula.quatember@oeai.at

Mag. Dr. Alice Waldner

Institut für Kulturgeschichte der Antike, Österreichische Akademie der Wissenschaften, Bäckerstraße 13, A-1010 Wien E-Mail: alice.waldner@oeaw.ac.at

Mag. Dr. Matthias Pfisterer

Kunsthistorisches Museum, Burgring 5, A-1010 Wien

E-Mail: matthias.pfisterer@khm.at

Dr. Maria Aurenhammer

Österreichisches Archäologisches Institut, Franz Klein-Gasse 1, A-1190 Wien

E-Mail: maria.aurenhammer@oeai.at

Abbildungsnachweis: Abb. 1: Plan C. Kurtze; Abb. 2. 4. 5. 7. 9. 10. 12: U. Quatember; Abb. 6. 8. 11: S. Swientek; Abb. 13-24: A. Waldner/J. Struber; Abb. 3. 25-38: N. Gail; alle Abb. C ÖAI Wien. 